\title{
Visual Political Communication in Popular Chinese Television Series
}

\section{Florian Schneider}

A Thesis Submitted for the Degree of

Doctor of Philosophy

School of East Asian Studies

The University of Sheffield

August 2008 


\section{Abstract}

This thesis argues that the content of popular Chinese TV dramas helps construct and reenforce social and political reality in China by defining what is true. With the increasing liberalisation of the Chinese TV drama market, this construction process is becoming more and more complex: it is not merely influenced by the political interests of state and party institutions, but also by the commercial interests of producers and broadcasters, as well as by the viewing habits and interests of audiences. Consequently, Chinese TV dramas create ideas concerning Chinese society which are simultaneously popular and politically 'healthy'. Based on qualitative interviews with Chinese media experts and production crew members conducted in 2007, my research shows how various actors and institutional factors influence the production of political discourses in Chinese TV dramas. This thesis also offers a qualitative analysis of how the discourses on two political concepts (governance and security) are depicted in three particularly popular dramas, one historical epic, one crime drama, and one teen drama. This analysis shows that these programs all link their political message to patriotic sentiments or conservative gender discourses, and that this is not the result of political directives but instead of market dynamics and of audiences' viewing preferences. In this sense, the present research shows how the apparent liberalisation of the drama market in reality imposes a whole framework of new cultural, political, and economic restrictions, which in turn leads to the production of TV content that is firmly rooted in hegemonic discourses. This discourse is then not primarily reproduced because it is politically opportune, but because it is popular. 


\section{Table of Contents}

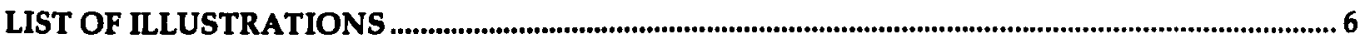

LIST OF ABBREVIATIONS ...................................................................................................................... 8

ACKNOWLEDGMENTS................................................................................................................................ 9

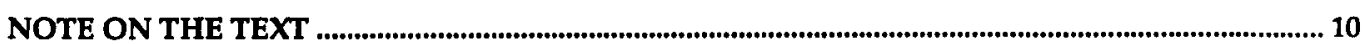

1 INTRODUCTION ...................................................................................................................................... 11

2 POPULAR TELEVISION ANALYSIS: RESEARCH, THEORY AND METHODOLOGY......... 15

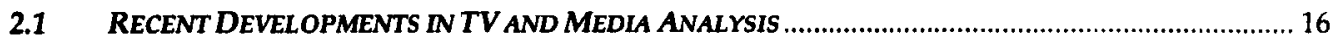

2.1.i The Relevance of Popular Culture and Visual Material in the Social Sciences ................. 16

2.1.ii Types of Analyses: Production, Product and Audiences..................................................... 18

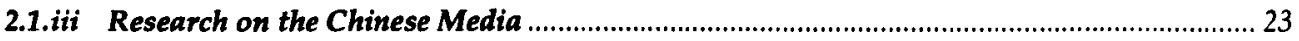

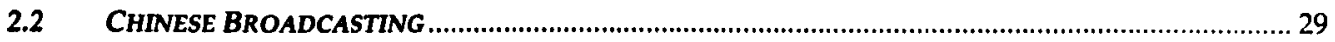

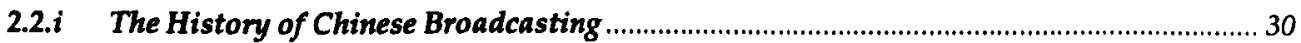

2.2.ii Structure of the Chinese Broadcasting System .................................................................. 33

2.2.iii The Broadcasting Market and its Primary Product: the TV Drama Series........................ 37

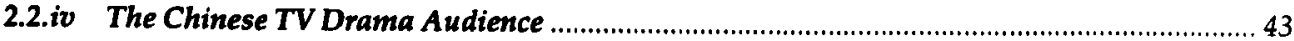

2.2.v The Debate: TV Content between Market Liberalisation and State Control ...................... 47

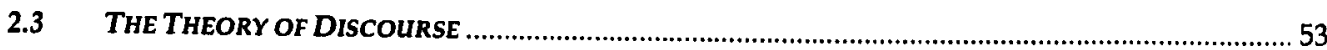

2.3.i Communication Practices and the Construction of Truth ….............................................5 53

2.3.ii The Anglo-Saxon Tradition of Critical Discourse Analysis .............................................. 56

2.3.iii The Post-Marxist Approach to Discourse Analysis............................................................... 60

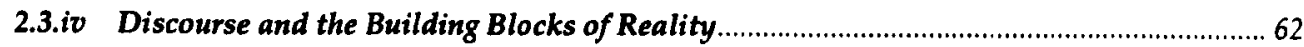

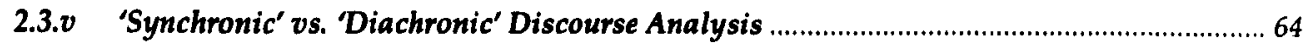

2.3.vi A Methodology of Critical Discourse Fine-Analysis ............................................................6

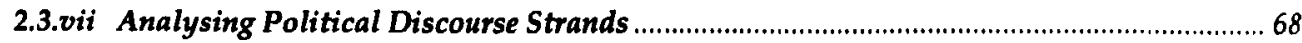

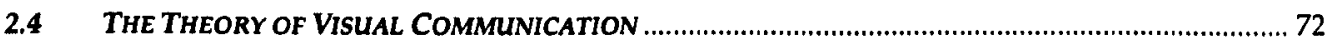

2.4.i Understanding Images as Purveyors of Meaning ….......................................................... 73

2.4.ii How Signs Work within Visual Communication .................................................................. 79

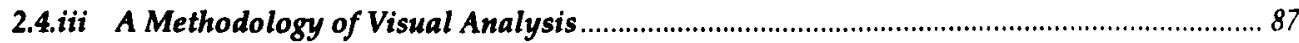


2.5 THE BASIS FOR THE PRODUCTION ANALYSIS: INTERVIEWS WITH CHINESE MEDLA EXPERTS .........96

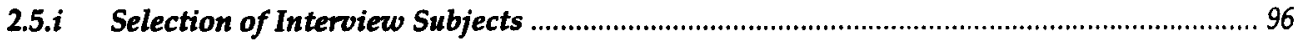

2.5.ii Research Procedure: Structure and Questions of the Qualitative Interviews................. 97

2.5.iii Processing, Analyzing and Referencing the Interview Material .................................... 99

2.5.iv Ethic Guidelines for Interview-Based Research........................................................ 100

2.6 THE BASIS FOR THE PRODUCT ANALYSIS: A CROSS-GENRE EXAMINATION OF ChINESE TELEVISION

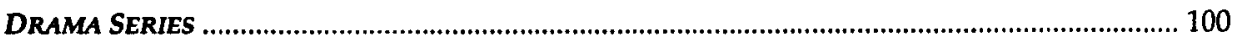

2.6.i The Selection of Representative Research Material .................................................. 101

2.6.ii Coding the Material for Political Discourses ........................................................... 103

2.7 SUMMARY: TOWARDS AN INIEGRATED METHODOLOGY OF CRITICAL VISUAL DISCOURSE

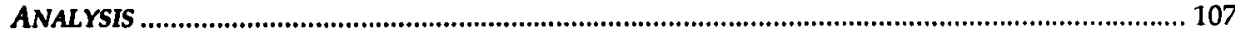

3 FACTORS IN THE CREATION OF CHINESE TV DRAMAS............................................... 110

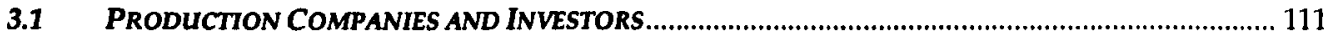

3.1.i State-Owned Production Companies ........................................................................ 111

3.1.ii Non-Govemmental Production Companies and the Risks of Drama Production ......... 112

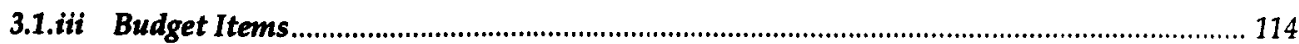

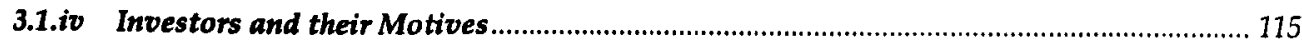

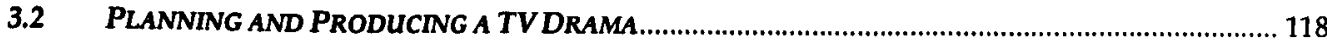

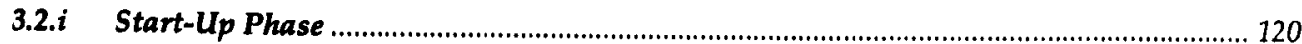

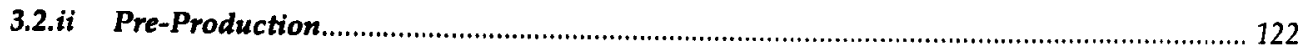

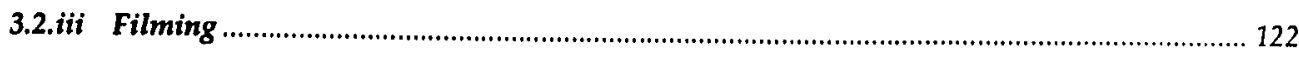

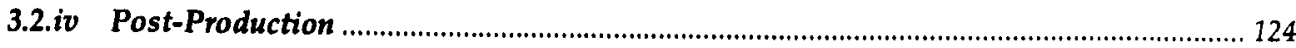

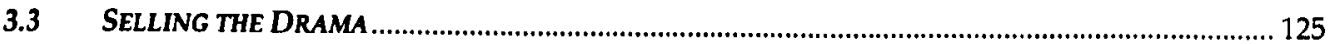

3.3.i Distribution: How TV Series Are Marketed............................................................ 125

3.3.ii The Buyers: TV Stations and their Motives .................................................................. 129

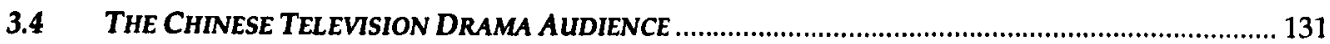

3.4.i Assessing Audiences: the Chinese Audience Measurement System ............................. 131

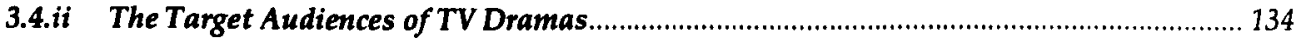

3.4.iii Viewing Habits: How Chinese Television Dramas Are Watched ................................... 135

3.4.iv Drama Genres: What Audiences Watch ................................................................. 138

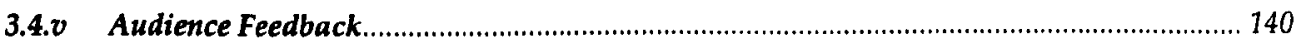


3.5.i Important Govemment and Party Institutions ..................................................... 148

3.5.ii The Tools Used to Control Television Drama Content ............................................... 154

3.5.iii The Guidelines Governing the Censorship Process ................................................ 157

3.6 THE CHINESE PERSPECTIVE ON THE INFL UENCE OF TELEVISION …....................................... 163

3.6.i The Official Position: Media Content's Impact on Viewers ......................................... 164

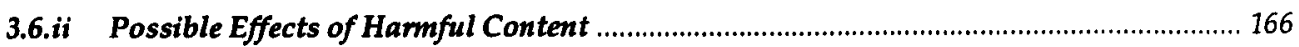

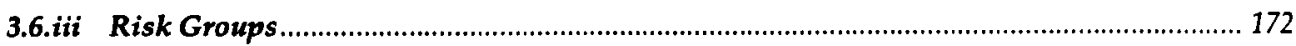

3.6.iv Justifying Censorship and Govemment Control in China ........................................... 174

3.6.v Putting the Official Position into Perspective: Society and the Viewers ...................... 177

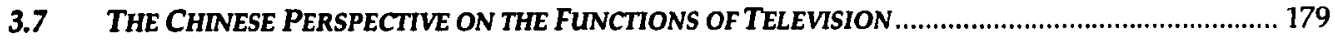

3.7.i The Official Position: TV Drama as a Propaganda and Education Tool ...................... 180

3.7.ii The Altemative Position: TV as an Entertainment Enterprise .................................... 182

3.7.iii Additional Functions: Psychological and Social Relevance of TV Drama .................... 185

3.8 SUMMARY: THE COMMERCLAL INTERESTS AND POLITICAL TARGETS OF TELEVISION ENTERTAINMENT.

4 CHINESE TV DRAMAS AND THEIR DISCURSIVE CONTENTS ........................................ 191

4.1 Visual Political Discourses in a Historical DRama .................................................... 191

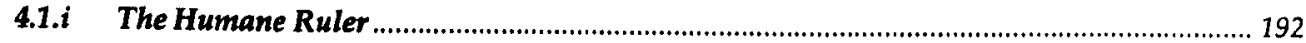

4.1.ii Foreign Security and the Establishment of International Relations ............................ 202

4.2 Visual Political Discourses in a Main-Melody CRIMe Drama ..................................... 234

4.2.i Responsible and Professional Leadership ................................................................. 235

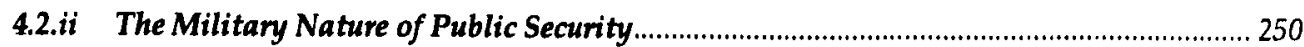

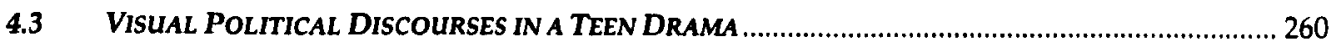

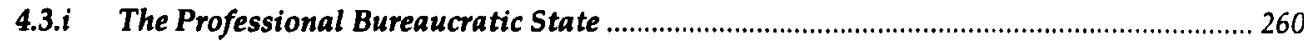

4.3.ii Security Issues as a Comical Plot Complication ........................................................... 269

4.4 Summary: Shaping Polttical Truths for a CONSERVATIVE TARget Audience ................. 285

5 CONCLUSION: CHINESE TV DISCOURSES AND THE FACTORS OF THEIR PRODUCTION 


\section{List of Illustrations}

Figure 1: Sample TV Schedule, Evening Programme for 1 february 2005 (TUeSday) ....................................... 41

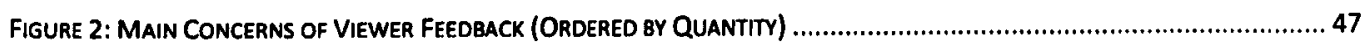

Figure 3: Sample Sequence Protocol from the Television Drama Series "The Commissioner 2" ............................. 91

Figure 4: Sample Shot Protocol from the Television Drama "The Great han Emperor Wu”................................99

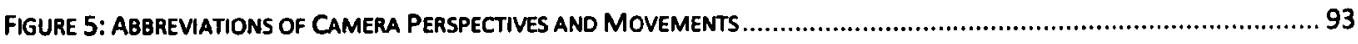

FIGURE 6: FreQUENCY GRAPHIC "BERLIN: SYMPHONY Of A GREAT CIT" (KORTE, 1999, 86) .......................................94

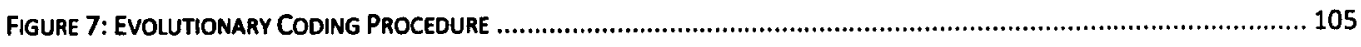

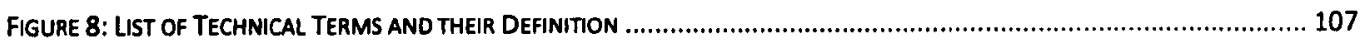

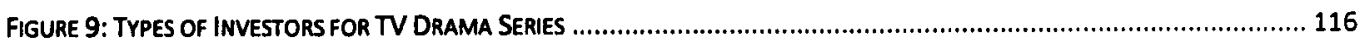

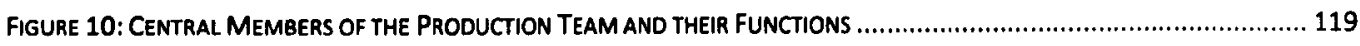

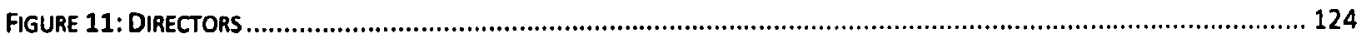

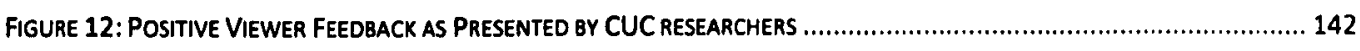

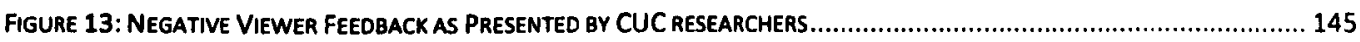

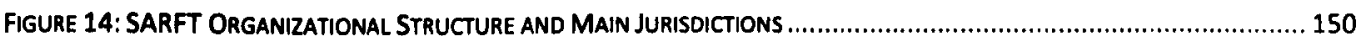

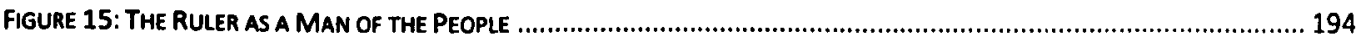

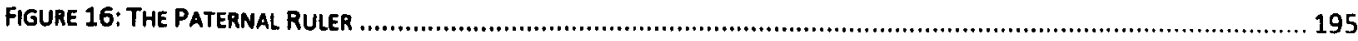

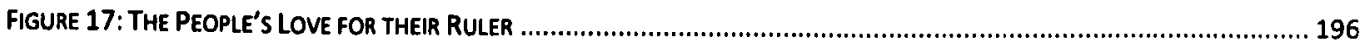

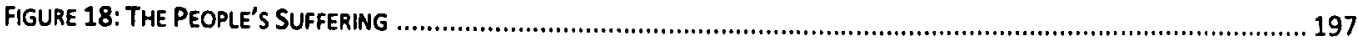

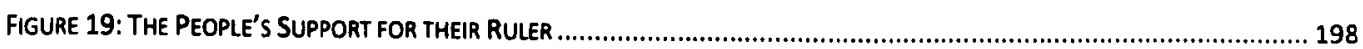

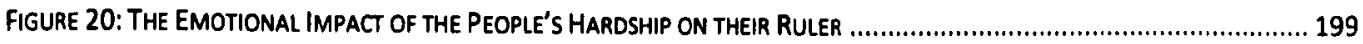

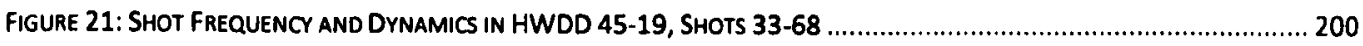

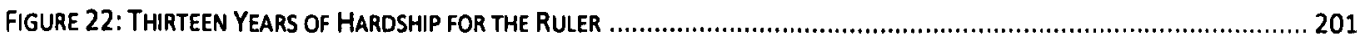

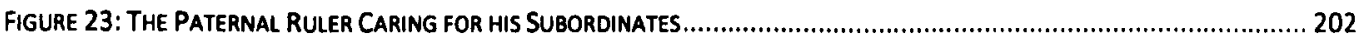

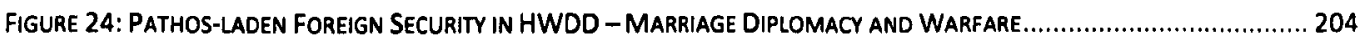

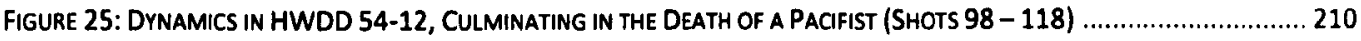

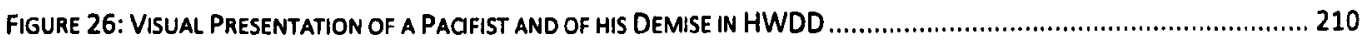

Figure 27: The EXotic COMmodities of Han's Central ASIAN Neighbours (HWDD 44-01) ................................... 220

Figure 28: Establishing Trade Relations in an EXotic yet Friendiy SURrounding (HWDD 44-02) ......................... 222 
Figure 29: Dynamics of Zhang Qian Convincing the QueEn In HWDD 44-03 (ShOts 25 - 42).

FIGURE 30: ZHANG QIAN CONJURES UP IMAGES OF WAR TO CONVINCE A PACIFIST (HWDD 44-03)

Figure 31: The Debate on War and Peace at the indo-SCrth Court (HWDD 44-07). 225

Figure 32: Emotive Imagery to Instill National Pride in the Viewers.

Figure 33: Patriotic IMAgery in the final Sequence of HWDD.

figure 34: Secretary Cheng from the CCP Assesses a Hostage Situation

Figure 35: Conventional VISUALIzATION OF Dialogues in GAJZ.

Figure 36: Discourse Fragments in the Dialogues between Commissioner and Secretary.

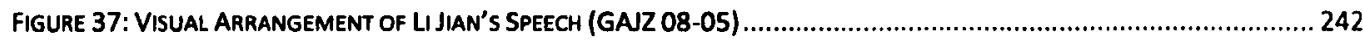

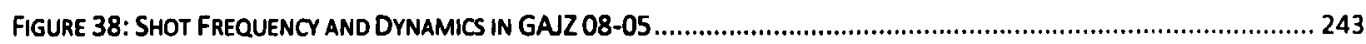

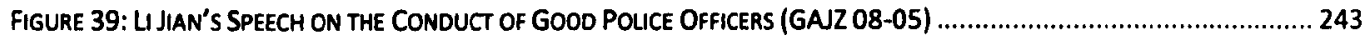

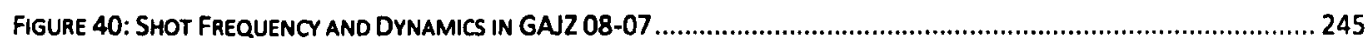

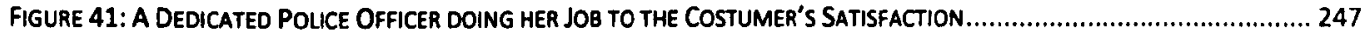

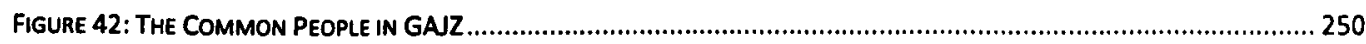

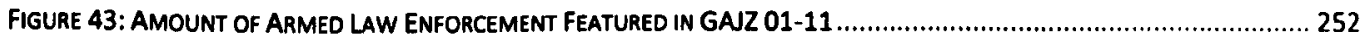

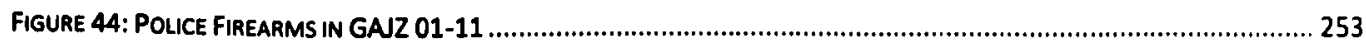

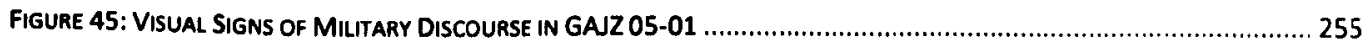

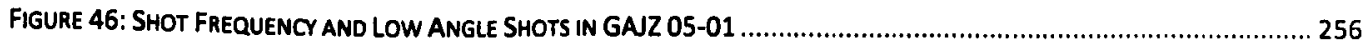

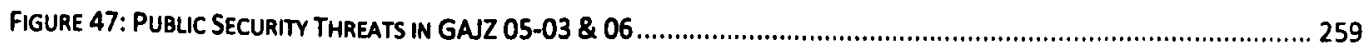

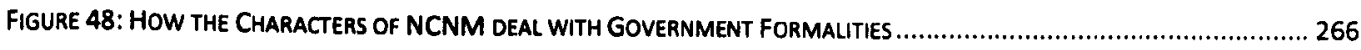

Figure 49: A Pregnant Woman's Administrative Procedoures Resolved With Male Assistance .............................2 268

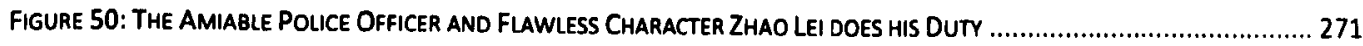

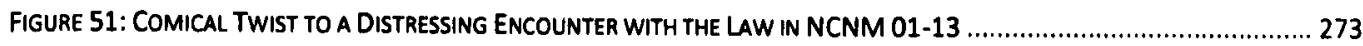

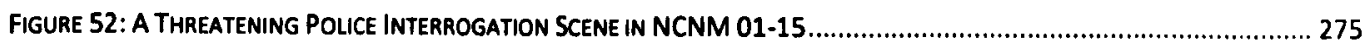

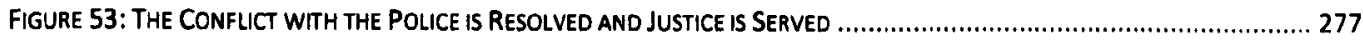

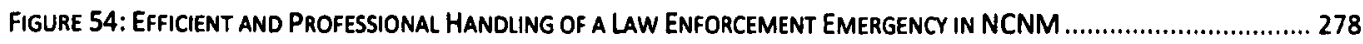

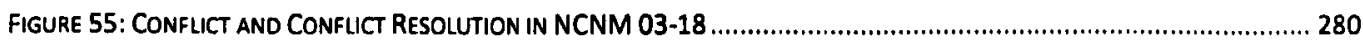

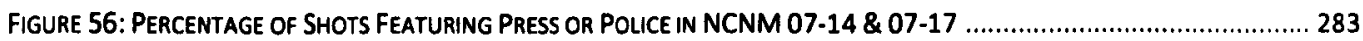

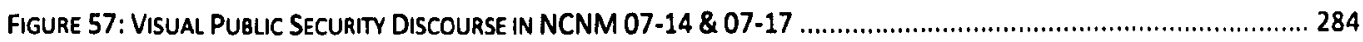




\section{List of Abbreviations}

BEV:

BTV:

CCP:

CCTV:

CSM:

$\mathrm{CU}$ :

CUC:

DA:

ECU:

ELA:

ELS:

$F(+,-)$ :

GAJZ:

HA:

$\mathrm{HC}$ :

HWDD:

LA:

LS:

$M(l, r, u, d, f, b)$

MC:

MCU:

MEI:

MII:

MPT:

MRFT:

MS:

NCNM:

OTS:

OTV:

$P(l, r)$ :

POV:

PRC:

RMB:

RMRB:

SARFT:

SPPA:

STV:

$T(u, d):$

TNS:

WTO:

$Z(+,-)$ : bird's-eye view (a type of camera angle)

Beijing Television

Chinese Communist Party

China Central Television

CVSC Sofres Media (an audience research company)

close-up (a type of camera shot)

Communication University of China

Dutch angle (a camera angle)

extreme close-up (a type of camera shot)

eye-level angle (a type of camera angle)

extreme long shot (a type of camera shot)

into or out of focus (a type of camera action)

Gonanju Zhang 2 (Chinese television drama series)

high angle (a type of camera angle)

hand camera (a filming technique)

Han Wu Da Di (Chinese television drama series)

low angle (a type of camera angle)

long shot (a type of camera shot)

movement left, right, up, down, forward or backward (types of camera

action)

Ministry of Culture

medium close-up (a type of camera shot)

Ministry of the Electronics Industry

Ministry of the Information Industry

Ministry of Post and Telecommunication

Ministry of Radio, Film and Television

medium shot (a type of camera shot)

Nancai Numao (Chinese television drama series)

over-the-shoulder (a type of camera angle)

Oriental Television (Shanghai-based TV station)

pan left or right (a type of camera action)

point-of-view (a type of camera angle)

People's Republic of China

Renminbi (PRC currency: the Chinese Yuan)

Renmin Ribao (the People's Daily newspaper)

State Administration for Radio, Film and Television

State Press and Publication Administration

Shanghai Television

tilt up or down (a type of camera action)

Taylor Nelson Sofres (an audience research company)

World Trade Organization

zoom in or out (a type of camera action) 


\section{Acknowledgments}

There are numerous institutions without which this research project would not have been possible, and a number of people to whom I owe personal thanks.

The University of Sheffield supported this project with an incentive award during the first year. The field research that followed during the second year was financed by the Universities' China Committee in London. The Fudan University in Shanghai assisted with visa applications and other formalities throughout my stay in China.

I am particularly grateful to my two supervisors, Lili Chen and Hugo Dobson, for their continuous constructive criticism and advice throughout the three years of this project. I would further like to express my thanks to the other faculty members and the office staff at the School of East Asian Studies in Sheffield for offering me a chance to teach throughout this time, and for their support over the last three years. In addition, I would like to express my gratitude to Michael Friedrich at the Asia Africa Institute in Hamburg for providing valuable contacts in China, and to Marion Muller for inspiring this research project.

I owe special thanks to the media experts who volunteered to participate in the qualitative interviews of this thesis. Their honest and detailed explanations have contributed greatly to my understanding of the Chinese television drama market, and their hospitality made my research stay in China a very pleasurable experience.

Finally, I would like to thank my family and friends for being patient with me for so long, and for supporting me on this journey.

The analyses and interpretations presented in the following text are of course entirely my own, and the conclusions reached in this thesis reflect my opinions, not those of the persons mentioned above. 


\section{Note on the text}

Throughout the following text, all Chinese characters are printed in their simplified form. All transliterations of Chinese names and phrases follow the Hanyu Pinyin system, and are, where applicable, placed in cursive script before the Chinese characters.

The bibliography to this thesis references all Chinese works in simplified characters rather than transliterated form, and provides the Chinese script for the authors' names and publishing houses as well. This is done in order to eliminate any ambiguities that the Pinyin transliteration would create, and to enable readers knowledgeable of the Chinese language to correctly identify the author, the work, and the publisher in question. For readers who are not knowledgeable of the Chinese language, a translation of each work's title is provided in brackets after the original Chinese name of the work.

The sections in this thesis that analyse qualitative interviews (Chapter 3) and visual materials (Chapter 4) use a special referencing system, which is explained in the sections that deal with the respective research methodologies (sections 2.5 and 2.6). 


\section{Introduction}

In the People's Republic of China (PRC), television has become arguably the most influential information medium: with nearly every urban family now having access to a television set (and therefore access to nationwide news-coverage and entertainment), the success of this medium has prompted media analysts to retrospectively call the introduction of television to the large cities of China in the 1980s "the most important cultural event since the Cultural Revolution" (Allen, 1992b, 2).

But television is not only relevant because of its rapid spread in this nation of 1.3 billion inhabitants. To researchers in the field of Chinese Studies, the analysis of popular television promises to expose how political messages are communicated to Chinese audiences, or as one German media researcher states (Kramer, 2004, 39; author's translation):

...television has adopted the function of an announcement medium, i.e. the function of communicating the official discourses and, through the effects its narratives and images have on emotions and identifications, of controlling not only mere information but also the self-perception of its recipients, that is to say: of the entire Chinese population.

Other observers, such as the Hong Kong journalist Yan Hua, follow a similar argument when they observe that in particular popular television drama series are not solely an entertainment format, but more importantly a crucial propaganda tool of the Chinese leadership (Yan, 2005). According to Yan's assessment of recent historical costume dramas, the depiction of various Chinese emperors as benevolent rulers functions as an allegory, indirectly propagating the legitimacy of the Communist Party's rule over China: "President Hu Jintao thus is a good emperor - it's clear from prime-time TV" (ibid.).

Such observations are made before a backdrop of ongoing reform in the PRC: numerous researchers have pointed out that the Chinese media market, including the market segment of television drama production and broadcasting, has become more and more liberalised over the past decade and is today primarily a profit-driven industry (Griffiths, 2005; Zhang et 
al., 2005). This contradiction between market liberalisation and political control in the broadcasting sector poses a challenge to our understanding of popular entertainment in the PRC. The question is: does a format such as the television drama serial function as an entertainment enterprise, as a political propaganda tool, or possibly as a mixture of both?

The present thesis attempts to answer this question by qualitatively analysing what factors influence the production of popular Chinese television drama series, and what political discourses are presented in such programmes. In order to do so, this project approaches television dramas from two angles:

First, the conditions under which television drama series are produced and broadcasted are analysed. This was achieved by conducting fourteen qualitative interviews with Chinese media experts about their experiences with this particular entertainment format and the factors that they believed influenced its production. The research subjects included producers, directors, screenwriters, editors, broadcasting directors as well as Chinese university researchers involved in the analysis of the Chinese TV drama market.

This production-side analysis is followed by the main section of the thesis: a visual analysis of three television drama series, aimed at establishing what discourses different types of dramas communicate. The three programmes are: the historical epic $\mathrm{Han} W u \mathrm{Da} D i$ 汉武大帝 ("The Great Emperor Wu of the Han Dynasty"; in the following referred to as HWDD), the state-commissioned crime drama Gonganju Zhang 2 公安局长二("The Commissioner - Part 2"; GAJZ), and the teen drama Nancai Nümao 男才女貌 ("Talented Man and Beautiful Woman"; NCNM). All series were aired on China Central Television between 2003 and 2005 , and achieving audience ratings that made them rank among the twenty most popular TV dramas in the annual statistics (Wang, 2004; 2005; 2006). The analysis of these programmes combines a critical discourse analysis approach with a visual research methodology that is 
based on semiotic theory. It applies analytical tools such as sequence protocols, shot protocols, transcripts and shot graphics in order to isolate how meaning is communicated through various visual, acoustic and verbal markers (so called 'signs'). In a next step, the analysis combines these markers in order to establish what statements they make concerning two political issues (or 'discourse strands'): governance and security.

A number of issues are not addressed in this thesis: first, it should be pointed out that this research project only examines the discourses presented in television dramas that were aired on national television. Popular programmes that were created for provincial, municipal or satellite stations were not examined in this analysis, seeing as such programmes are aimed at very specific groups of viewers, and not at the standard national target audience. In addition, entertainment formats other than drama series (for instance game shows or talk shows) have also been excluded from the analysis, based on the observation that drama series currently constitute the most influential entertainment format on Chinese television (see Section 2.2.iii). Finally, the present project does not include an analysis of poplar entertainment's effect on Chinese audiences. The way in which production crew's conceptions of a target audience influence the creation of content is examined (Section 3.4), but no interviews or surveys were conducted for this research that focused on the actual viewers of such programmes. Such analyses have, however, been conducted by other researchers, and the merits and flaws of audience-centred approaches are reviewed in Chapter 2 (Sections 2.1.ii and 2.2.iv).

This thesis is structured as follows: Chapter 2 offers first a review of the types of research that have been conducted on media content, and particularly on television entertainment formats (2.1). It then gives an overview of the Chinese broadcasting sector and summarises what issues recent research has raised concerning television entertainment's function in the PRC (2.2). Next, the chapter introduces the theoretical and methodological concepts used in this thesis, i.e. the critical discourse analysis approach (2.3) as well as the visual analytical 
tools (2.4) which are later applied in the analysis of the three television drama series. Finally, the research procedures for both the qualitative interviews (2.5) and the visual analysis (2.6) are explained.

Chapter 3 presents the findings of the qualitative interview analysis and deals with the various factors that the interview subjects believed influenced the production of television drama content. The chapter first examines what organizations can create television dramas, and how they procure their funding (3.1). It then analyses the different phases of television drama production and establishes which production crew members are responsible for which part of the content production process (3.2). Section 3.3 examines how television drama series are marketed, sold and then broadcasted. The subsequent section analyses which role preconceived notions of a Chinese TV drama target audience play in the production of such programmes (3.4). Next, the question of how the authorities influence the production and broadcasting of television content is addressed (3.5). The final two sections of the chapter examine how the media experts who were interviewed for this project explained the relevance of television entertainment in China: Section 3.6 analyses the interviewees' views on the influence that television has on Chinese viewers, and Section 3.7 examines what conceptions the research subjects hold concerning the functions that TV plays in the PRC.

Chapter 4 presents the results of the visual analysis, and constitutes the central focus of this thesis. The first section examines the television drama HWDD (4.1), the second the programme GAJZ (4.2), and the final section the series NCNM (4.3). In each case, the statements that are made concerning the themes of governance and security are analysed.

The central issues and findings of each chapter are presented in respective summaries (2.7; $3.8 ; 4.4)$, and the research results are brought together in the final conclusion (Chapter 5). The appendix is provided in a separate volume, which includes a DVD with the visual data. 


\section{Popular Television Analysis: Research, Theory and Methodology}

The aim of this research project is to undertake a visual political analysis of popular Chinese television series. Although there are numerous approaches and examples on how to analyse media products in general and the medium of television drama serials in particular, there is no consistent and comprehensive methodological framework that such research follows (see, for example, the various contributions in Allen, 1992b and 1995). In this chapter I would therefore like to first offer an overview of the relevant literature that deals with the research of media products, both in the Chinese context and in other societies (Section 2.1), and then summarise what is currently known about broadcasting in the PRC (Section 2.2). I shall then turn to the theory and methodology relevant to my research: first the critical discourse approach that forms the basis for this project (Section 2.3), then the visual political communication approach I am adopting to examine visual material (Section 2.4). My aim is to show how these two research approaches are compatible, and how the combination of the two offers a comprehensive methodology for the analysis of political discourses in popular television products.

Since the analysis presented in this thesis focuses on two aspects of Chinese television dramas (the general circumstances of TV drama production as well as the content of three specific series), the final two sections of this chapter explain what criteria and procedures were adopted in each of these cases: Section 2.5 summarises the procedure of selecting and interviewing Chinese media experts, while Section 2.6 explains the process of selecting and analysing the three particular television drama series. 


\subsection{Recent Developments in TV and Media Analysis}

The analysis of popular media content and visual communication practices is a broad, interdisciplinary research topic, which has been approached by analysts from various fields. The following section provides an overview of these fields (2.1.i), a summary of research that focuses on media products, particular visual materials (2.1.ii), and a review of the work that has in this respect been conducted in the Chinese context (2.1.iii).

\subsection{The Relevance of Popular Culture and Visual Material in the Social Sciences}

The study of visual material traditionally falls into the realm of art historical research, concentrating largely on the fine arts, on architecture, sculpting and painting (Ellenius, 1998; Panofsky, 1987). It has not been until most recently that researchers from the social sciences have come to recognise to what extent visual communication offers insight into the problems of their specific fields: Today, the analysis of visual material provides a valuable tool to researchers in fields as diverse as political science, media studies, cultural studies, psychology, or history, to name just a few (an overview of how visual material is analysed in these fields is provide in Muller, 2003, 121-266). Particularly researchers in the cultural studies (Hall, 1980; Kellner, 1995) and political scientists (Leggewie, 1994; Müller, 1999; Warnke, 1994) have developed ways to combine traditional iconography (i.e. the analysis of images and their meaning in their respective social and cultural contexts; Müller, 2003, 249256) with political and sociological questions, for example how images are used to purvey political messages (Warnke, 1994), or what role images of politicians play in electoral campaigns (Müller, 1997a; Müller, 1997b).

Political imagery in movies and the print media has received considerable attention from social scientists. Examples include the various analyses in Hoffmann (1999), which focus on the question of how authority is portrayed visually, as well as Marion Muller's examination of the imagery in the German tabloid BILD (Muller, 2003; see also Section 2.4). However, the 
analysis of popular television formats such as soap operas, sport events, music videos, talk shows or game shows is mostly conducted by cultural studies researchers (take for example the analyses presented in Allen, 1995; Craig et al., 2002; and Donald et al., 2002c). To most social science scholars the seeming triviality of such materials appears to offer little, if any, explanatory value in regard to issues of society, politics, international relations, or economics (Allen, 1992b, 3-4). Robert Allen, Professor of Radio, Television, and Motion Pictures at the University of North Carolina, aptly describes this problem when he concedes "that we don't regard many of the programmes we watch as serious, consequential, or important. They're not 'supposed' to be taken seriously, and they certainly don't seem to require close analysis to be comprehended or made enjoyable" (ibid.). This reluctance to attribute scientific relevance to popular visual communication is further aggravated by aesthetical and traditional cultural notions that popular television formats are of less quality than established art forms, such as oil paintings on canvas or motion pictures (Allen, 1995, 3). Entertainment formats such as soap operas or sit-coms are often depreciated as part of a "lower" culture and are regarded as "dirt" (ibid.). In the wake of such preconceptions, the idea that popular television programmes may offer insights into political discourses and popular sentiment particularly because of their popularity is often overlooked: Chris Barker from the University of Wollongong in Australia sums this problem up when he writes (Barker, 1997, 218):

...it makes little sense to discuss whether television is 'good' or 'bad' in an abstract aesthetic manner, rather, we need to consider, from a political and inevitably value-laden position, the ideological construction and potential consequences of television.

As shall be shown below, the popular format of television drama series is a major influence on culture and society in the PRC, and deserves attentions from social scientists and researchers in Chinese Studies alike (2.2.iii). 


\subsection{Types of Analyses: Production, Product and Audiences}

As Marion Müller has points out, analyses of visual material broadly fall into at least one of three categories (Müller, 2003, 15-17):

1. Production analyses: studies that focus on the production processes of visual material, as well as on the political-economic dynamics within the media sector that influenced this process.

2. Product analyses: research that examines the content, narrative and central themes of specific materials.

3. Effect analyses: works that examine how audiences perceive visual materials.

In the area of production analyses, researchers are usually concerned primarily with market developments and the procedures involved in producing television programmes. Since television, as "a modern industrial organization", rarely possesses a distinct "author" (Allen, $1992 b, 9)$, such analyses are often driven by theories and methodologies derived from economics, administration theory or political science. Examples of this in the Chinese context (and not necessarily with a focus on TV) are the contributions in Lee (2003a), which analyse the effects that globalization dynamics and China's accesșion to the World Trade Organization (WTO) has had on the domestic media market (Chan, 2003; McCormick et al., 2003; Zhao, 2003), arguing that the introduction of new technologies and a new business culture has opened up new spaces for the construction of discourses in the "public sphere" (McCormick et al., 2003). The aim of such research, whether in the Chinese case or elsewhere, is usually to shed light on the "formal and informal rules regarding who has access to the means of production and distribution", for example "the laws of the land in areas such as libel, obscenity, public order, ownership and free speech" (Barker, 1997, 28). In some cases, analyses follow the production of one particular media product, for example Michael James Intintoli's work on the production process of the soap opera Guiding Light (Intintoli, 1984). 
In product analyses of visual material, authors commonly draw their theoretical and methodological approaches from disciplines such as film studies (Borstnar, 2002; Faulstich, 2002; Korte, 1999; Mikos, 2003), literature and narrative studies (Kozloff, 1992), psychology (Flitterman-Lewis, 1992), iconography (Muller, 1999), cultural studies (Fiske, 1992), semiotics (Seiter, 1992) or gender studies (Kaplan, 1992; Brunsdon, 1995). ${ }^{1}$ Often such research is augmented by discourse theory in the tradition of Michel Foucault, meaning that the authors regard television as a communication practice that constitutes the social and political reality that people live in (for examples see Allen, 1992b and Kramer, 2004; the discourse analytical approach used in the present research project follows a similar logic and is explained in chapter 2.3). Critical discourse analytical approaches are particularly common in analyses of television drama series: a common argument is that this particular programme format constitutes "a model of 'how we live' and is talked about in civil society as much, if not more, than the news", making it detrimental to the construction of the reality we live in (Barker, 1997, 225). However, in many cases the work of researchers examining television drama content is confined to a specific set of problems; the central research questions that are usually asked are (ibid., 118):

1. What is the aesthetical or cultural 'quality' of soap operas?

2. Is the main target group of this specific format a female audience?

3. What gender issues are raised in these programmes?

4. Does the popularity of Western soap operas such as Dallas, Dynasty or Neighbours and their export to foreign markets facilitate cultural imperialism? (For such an argument see in particular Hagedorn, 1995)

5. What are the similarities and differences in productions from different countries?

This last question is addressed particularly in case-studies of Latin American telenovelas, such as the ones conducted by Ana Lopez and Jesús Martin-Barbero. Lopez analyses the

${ }^{1}$ That these theoretical and methodological approaches of western scholars are perceived by Chinese intellectuals is shown in Zhang Yingjin's article (2004) in the Journal of the Beijing Film Academy (北京出欵院学: 报). 
genre of the telenovela in comparison to the US soap opera and shows that whereas the North American format is constructed in a way that mainly targets female viewers, the South American format of the telenovela is aimed at viewers of a certain (upper) class background (Lopez, 1995, 260-261). Martin-Barbero further shows that the way in which this local format reproduces specific Latin-American cultural traditions (such as melodramatic narratives, a focus on kinship, or particular types of satire) has enabled the telenovela to replace USAmerican soap operas in the Latin-American broadcasting market (Martín-Barbero, 1995). Both Martin-Barbero and Lopez argue that in order to satisfy such a large market, the telenovela format has been moving away from promoting national themes to advocating a form of pan-nationalism (Lopez, 1995, 270) and Latin American integration (Martín-Barbero, 1995, 283). Another contribution to cross-cultural research on soap operas is Gabriele Kreutzner and Ellen Seiter's comparison of the American series Dallas and Dynasty with the popular German programme Die Schwarzwaldklinik (The Black Forest Clinic), in which the researchers analyse and contrast the respective programmes from a gender studies perspective (Kreutzner et al., 1995).

Whereas the above analyses are primarily concerned with cultural issues, there are studies that address more explicitly political questions: for instance, Alison Griffiths explores the role that a particular Welsh-language soap opera plays in the formation of the national or cultural identity of Welsh viewers (Griffiths, 1995). A similar perspective is adopted by Lisa Rofel from the University of California in Santa Cruz: Rofel analyses how the Chinese drama series Yearnings aimed at constructing a mutual Chinese identity (Rofel, 1995; see also section 2.1.iii). Other studies focus on the way that contemporary discourses are depicted in soap operas: For example, Christine Geraghty's research is concerned with the question of how social issues are presented and discussed in UK soap operas (Geraghty, 1995), and Joy Fuqua 
(1995) examines how homosexuality and AIDS are portrayed in the soap opera One Life to Live.

The central issues of effect analyses, i.e. the question of how who watches what, is mostly addressed by market researchers, psychological studies and research conducted in the cultural studies. As Chris Barker shows in his introduction to global television research (Barker, 1997), market researchers usually use quantitative data such as TV ratings and demographic statistics (sometimes combined with qualitative information concerning the preferences of certain target audiences) to provide advertisers and broadcasters with information concerning the popularity of certain programmes or programme formats. Examples of such surveys are discussed below, in Section 2.2.iv. An effect study that attempts to provide statistical data of its own is Stefan Kramer's analysis of the Chinese television audience (Kramer, 2006). It is discussed in Section 2.1.iii.

Psychologists, on the other hand, usually try to determine what effect television has on viewers' minds, and how visual information influences behaviour and perceptions, both consciously and unconsciously. A good example of this research is the work by Sandy Flitterman-Lewis (1992), who shows how the viewing of soap operas works at a subconscious level to create desires in viewers, make the viewing experience pleasurable, and create ways for the audience to identify with certain characters. A broad overview on audience research in the field of psychology is offered by John Condry (1989). Although such approaches are valuable within their particular scientific field, they have little explanatory power when it comes to the content of visual political communication and the way in which such content is produced. For this reason psychological approaches shall not be reviewed here further.

Another approach to the effect side of media consumption is the so-called "reader-oriented criticism", which is often adopted by authors from the discipline of cultural studies, such as 
Richard Allen (1992a): Allen attempts to explain how television 'addresses' the audience and how the so-called 'readers' of television infer meaning from particular programmes (ibid.). Similar to Flitterman-Lewis' psychoanalytical analysis (1992), Allen's intention is to show how viewers derive pleasure from watching popular television programmes such as soap operas and game shows. In order to do so, Allen draws from literary theory and treats television as 'text'; this enables Allen to highlight how viewers fill in the 'gaps' in television plots, and how they derive a coherent narrative from the generally elliptic formats on TV, just like the readers of a novel would (Allen, 1992a, 102-113). Another author who similarly focuses on how specific viewers 'read' popular television is Kate Baldwin (1995). Baldwin examines viewers' reception of a Latin American telenovela in a foreign cultural context (the reaction of Russian audiences to the Mexican programme Los Ricos También Lloran, The Rich Also $\mathrm{Cry}$ ) and argues that the melodramatic narrative format of that particular telenovela was so popular in Russian urban centres because it tapped into the reading habits of its audience, who had up to that time (the 1990s) been occupied primarily with narratives in the printed form (ibid., 288).

While such literary-studies approaches help us to understand how narratives function in various formats (on television as much as in novels), the general treatment of television as 'text' also poses a problem, which Allen himself summarises as follows (Allen, 1992a, 127):

...there is a danger in discussing the role of the television viewer in terms of the role of the literary reader (...). The danger, rather obviously, is that such a discussion obscures important differences between watching television and reading a book.

Since such approaches neglect to take into account the full range of modes in which television functions as a communication practice, I will not follow up on this specific strand of research here. Instead, I provide a research approach below that does not treat visual materials as text, but rather as a complex interaction of signs in different modes (Chapter 2.3). 


\section{1.iii Research on the Chinese Media}

In China, the relatively young commercial media market has drawn the attention of numerous authors, focusing both on print and visual media (Lee, 2003a). With regard to production analyses, contributions focus on such diverse topics as the influence of globalization on media technology, media content, and the public sphere in China (McCormick et al., 2003), cross-market analyses of administrative restrictions on the media market (Chan, 2003), comparisons of the mainland film market with the markets of Taiwan and Hong Kong (Curtin, 2003), the implications of opening up China's media sector to foreign investors (He, 2003) and to foreign programming content (Hong, 1998), the introduction of recent media reforms in China (Pan et al., 2003), the liberalization of China's newspaper market (Zhao, 2000), or the interests of different Chinese social and political groups in this rapidly growing sector (Lee, 2003b).

Particularly noteworthy is the work of media expert Zhao Yuezhi (1998), who examines the reforms of the media system and of journalistic practices in the PRC during the 80s and early 90s. Even though her focus is mainly on the evolution of newspaper journalism, her analysis of China's news system also encompasses radio and television programming (ibid., 94-126). Zhao's work shows how news production is caught between commercial pressures and political directives. She challenges claims that the move towards more audience-friendly content automatically leads to a democratization of the media (ibid., 185-189). By providing illustrative examples from her interviews with Chinese journalists and from her analysis of news content, Zhao demonstrates that Chinese journalism in the reform era has in many cases not overcome the political guidance of the $\mathrm{CCP}$, but has instead merely replaced it with a commercial market logic that has led to wide-spread corruption (ibid., 72-76), sensationalist marketing (ibid., 136), irresponsible and false reporting (ibid., 76-81), and a new commercially inspired form of self-censorship (ibid., 160). Her conclusion is that these developments are highly damaging to the credibility of Chinese journalism, and that only a 
comprehensive reform of China's political system and Chinese political communication processes will be able to create media that truly represent the Chinese citizen (ibid., 192). Although Zhao's analysis focuses on the news media, and only on developments up to the mid-90s, her analysis nonetheless raise a central question, which shall be addressed more fully below (see Chapter 3): does the market reform of media formats in China conflict with political directives of Party and State, or does the market logic in fact fuse with (and possibly facilitate) political mechanisms geared towards controlling media content (ibid., 159; for a similar argument see also Zhao, 1999)?

Another contribution that explicitly deals with Chinese television is Stefan Kramer's extensive analysis of the conflict between 'local' and 'global' aspects in the modern Chinese broadcasting system (Kramer, 2004, 55). Kramer argues that television is the most important medium for constructing Chinese reality and identity today (ibid., 144). At the heart of Kramer's study lies the question of how 'Chinese' television in the People's Republic actually is: Kramer argues that China does not share the western concepts of communication, imagery, and reality from which the western media technologies have historically been derived, and that the medium of television is therefore a foreign import that has no basis in Chinese culture (ibid., 61); he concludes that Chinese society has proven highly adaptive in tailoring the 'apparatuses' (i.e. the instruments) of this western technology to its local needs, creating programmes that communicate distinctly Chinese discourses (ibid., 542-543).

In the Chinese literature, the production process of television programming is usually treated by teaching manuals that describe what issues directors and producers should consider in their work, and that are aimed at preparing students of such trades for university examinations (see in particular Chen et al., 2005 as well as Huang, 2005; for a similar teaching manual that deals with the movie industry see Huang et al., 2006). For this purpose, such books include practical advice on how to write script outlines (Chen et al., 2005, 83-95 and 
177-178), calculate production costs (ibid., 208-220), write an investment plan (ibid., 274-277), or film and edit visual material (Huang, 2005), and provide information on what legal issues a television production might be confronted with (Lu et al., 2002, 309-323; Wei et al., 2005).

When it comes to product analyses, research of media discourses in Chinese Studies has mostly focused on the journalistic coverage of major events such as China's accession to the WTO in the year 2001 (Zhao, 2003), the impact of the terrorist attacks of 11 September 2001 on the nationalist sentiment of Chinese youths (Rosen, 2003), or the preparations for the 2008 Beijing Olympics (Polumbaum, 2003). When it comes to visual material, scholars of Chinese Studies are mostly concerned with popular mainland cinema (Cornelius et al., 2002; Lu et al., 2005; Zhang, 2002), avant-garde movies (Cui, 2005) or individual motion pictures by such influential Chinese directors as Zhang Yimou (Lu, 2005). Popular Chinese culture is rarely the focus of comprehensive political analysis. Instead, it seems, it is left up to journalistic authors to account for how pop is evolving and shaping modern China (Zha, 1995), and such authors do not apply a specific scientific approach to their analysis, much less a visual analytical methodology. In fact, to my knowledge there exist only two works in Western academia that specifically deal with soap operas in China from a scientific perspective:

1. One is the unpublished doctoral dissertation of Wang Yi (Wang Yi, 1996, 129-255) from Murdoch University (Perth, Australia). Wang's research includes, among other things, an analysis of the popular 1990 Chinese 'indoor soap' (shinei ju 室内剧) Expectations (Kewang 渴望). The analysis offers general television market statistics (production side), a view at the audience reception of the series and at press feedback (effect side), and a comparison of the major discourses within the soap on one hand and contemporary popular literature on the other (product side). The study does not, however, include a detailed analysis of the actual visual material, and is much rather an account of the series' narrative and its characters. 
2. The second paper is an article by Lisa Rofel from the University of California in Santa Cruz (Rofel, 1995), which also focuses on the series Kewang (though in Rofel's article the programme is translated as Yearning). Rofel's product analysis primarily looks at the functions that the melodramatic style of the series serves, and at the hegemonic discourse within the programmes narrative. Although the short article adopts a discourse analytical approach, it is, like Wang's work, lacking an in-depth visual analysis, and primarily deals with narrative issues.

In China, the topic of television drama content is actively discussed by many writers. However, none of the authors provide comprehensive visual analyses, and the focus of the research is again usually placed on the narrative and on character development; the questions such research is concerned with in the PRC is whether particular dramas are socially, politically and aesthetically acceptable (Zeng, 2002; 2005; and 2006), and what gender perspectives they promote (Dai, 2004, 222-267; Liu, 2005). They are, in that sense, very often television drama critiques rather than research into the functioning of television communication practices.

With respect to effect analyses of Chinese television, the most comprehensive work is arguably that by James Lull (1991). Lull not only provides background information on the development of the Chinese broadcasting market from the foundation of the PRC to the open-door policy of the 1980s (ibid., 1-29), but also offers a qualitative analysis of interviews with urban Chinese viewers that he conducted in 1986 (Lull interviewed eighty-five families in the four cities Shanghai, Beijing, Guangzhou, and Xi'an; ibid., 42). Through this analysis Lull shows on the one hand how Chinese families generally watch television (ibid., 59-91 and 154-181), and on the other hand what the interviewees' reactions to one particular television drama serial were (New Star, Xin Xing 新星, aired on CCTV in 1986; ibid., 92-126). In addition, Lull recounts how the Tiananmen demonstrations in 1989 were televised in the PRC (ibid., 
182-207). In this sense Lull's work is a stunning account of how a government-critical view pervaded the perceptions of Chinese audiences through the medium of television in the late 1980s, thereby undermining the authority of the Communist Party (ibid., 212) and leading to a sentiment of resistance that found its culminated in the Tiananmen demonstrations in 1989 (ibid., 218). However, despite these findings, Lull's analysis contributes little to the present research: due to its time frame, Lull's assessment of the Chinese broadcasting system is now largely outdated, and can serve only as a historical overview of the situation between 1949 and 1989. From a methodological perspective, Lull's focus on audiences also does not provide approaches that can be applied to the analysis of the political discourses in television drama series and the factors that lead to their production.

Another analysis of audiences was recently conducted by Stefan Kramer (2006). Kramer's project essentially emulates the approach taken by Lull two decades earlier, but also includes quantitative data. Kramer argues that Chinese television generally communicates a hegemonic discourse concerning the question of what it means to be Chinese, and that this hegemonic discourse essentially fuses various different concepts together (such as the state, the Communist Party, and Chinese national culture; ibid., 211). He further argues that even though this discourse has been internalised by many viewers, the medium of television nonetheless offers audiences the opportunity to reassemble such information, combine it with counter-hegemonic discourses, and apply it to the actual environment that the respective viewers live in (ibid., 219). In this sense Kramer sees television as a tool that has the potential of creating an active and emancipated Chinese audience that is able to free itself from the worldview that the authorities are trying to dictate (ibid., 220).

Although such arguments are highly instructive, Kramer's research is nonetheless problematic and should be treated with caution. This has several reasons: 
1. The author bases many of his findings on quantitative data, but uses a questionable statistical sample base: Kramer handed out questionnaires to 1,400 research subjects in Shanghai and Beijing, as well as to 1,400 research subjects in two selected counties in Hebei and Shanxi (ibid., 56). This essentially narrows the research project down to two urban environments and a very selective northern rural area. Kramer then combines his data with the data set of an entirely unrelated domestic project (without showing what the focus of that project was; ibid., 98) and uses the results to make generalised statements about all of China (for examples see ibid., 208 or 218-219).

2. Kramer misinterprets some of his quantitative data, for instance when he assesses that roughly twenty per cent of urban citizens receive their news information from TV, and then concludes that "television has by far taken over the function of the leading medium and most important method of entertainment" (thereby completely ignoring that, according to his own findings, radio, newspapers, and magazines each achieved similar statistical values as television did; Kramer, 2006, 94).

3. Kramer's research repeatedly refers to statistical material that is not referenced (for example when Kramer compares the Chinese situation with alleged conditions in Europe and the US; ibid., 85).

4. Finally, Kramer augments his quantitative research with qualitative data derived from interviews with Chinese viewers (ibid., 61-63). However, Kramer offers no information on how precisely these interviews were conducted or what questions were asked, and provides no quotes from or references to this qualitative data throughout his explanations.

Due to these various drawbacks in Kramer's approach, his data will not be used in the present research project. 
In the PRC, audience research is primarily conducted by two companies: AGB Nielsen and CSM (see Section 2.2.iv for a discussion of their work, and Section 3.4.i for an analysis of the effect that these statistics have on the television drama market). The statistical data on Chinese television audiences collected by CSM is published annually in the China TV Rating Yearbooks (Wang, 2004; 2005; 2006; 2007). In addition, a group of researchers from the Communication University of China (CUC) in Beijing is currently involved in an ongoing project to analyse the data provided by AGB Nielsen. Their goal is to establish what types of Chinese viewers watch what kinds of television drama series, and how they react to the programs on offer; the first of their annually planned evaluations has recently been published in Beijing ( $\mathrm{Li}, 2007)$. The project of the CUC researchers is reviewed (2.2.iv) and analysed (3.4.v) below.

\subsection{Chinese Broadcasting}

Before offering my own analysis of the factors that are relevant to the production of Chinese television drama series (Chapter 3), it is worth summarizing what other researchers have so far established concerning the workings of the Chinese broadcasting system, and what issues give rise to debate. This section therefore first gives an overview over the history of Chinese broadcasting (2.2.i), before then summarizing how the broadcasting sector itself is structured (2.2.ii). The following sections then examine what is known about the entertainment format of Chinese TV drama series (2.2.iii) as well as the audiences who watch it (2.2.iv). The final section focuses on two conflicting trends in the broadcasting sector (2.2.iv): the market liberalisations that have brought more and more freedoms to the system throughout the past two decades, and the ongoing attempts to nonetheless control that system and censor the content that is broadcasted. 


\section{2.i The History of Chinese Broadcasting}

When the first television stations were founded in the People's Republic of China in 1958 as part of the so called "Great Leap of Broadcasting Work" (Guangbo gongzuo dayuejin 广播工作 大跃进)2, Chinese television was still at an infant stage (Kramer, 2004, 86). The country possessed no content production facilities of its own and its TV broadcasting system was merely able to reach a small number of privileged cadres (ibid., 84-87). As Lull writes: "In these early years (..) almost no one had a television set with which to view the shows. Progress in the development of television hardware and software was slow" (Lull, 1991, 20). Today, the State Administration for Radio, Film and Television (Guojia guangbo dianying dianshi zongju 国家广播电影电视总局; or SARFT) claims that approximately ninety-four per cent of Chinese citizens had access to the nation's television programmes at the turn of the millennium (SARFT, 2002). The number of television stations is said to currently lie at roughly 2,000 (ibid.). ${ }^{3}$

In a 1983 declaration, the Chinese Communist Party (CCP) hailed television as the most important propaganda method for reaching Chinese households (Zhang et al., 2005, 36). Since then, television has arguably become the most dominant medium in modern China (ibid.; Kramer, 2006, 13). Nevertheless, the road to success for Chinese television has been a winding one.

While from the 1950 s to late 1970 s Chinese television was only a very nascent media format incapable of competing with more established information channels such as cinema and radio, the media environment experienced a massive period of change after the Cultural Revolution (Lull, 1991, 20-24). The rediscovery of television as a potential propaganda

\footnotetext{
${ }^{2}$ Beijing TV (Beiling dianshitai 北京电视台; or BTV) was founded on 1 Mai 1958, Shanghai TV (Shanghai dianshitai 上海电视台; or STV) on 1 October of the same year, and Harbin TV (Haerbin dianshitai 哈尔洴电枧台) on 20 December; for details see Kramer, 2004, 84-111.

${ }^{3}$ The exact number of television stations in China varies depending on the source. The SARFT recorded 1,984 stations for the year 2002 (SARFT, 2002). According to the estimates by Anke Redl and Rowan Simons, China may have possessed "more than 980 terrestrial and over 1,300 cable stations at provincial, city and (still) county level as well as over 30 provincial satellite channels" in that particular year (Redl et al., 2002, 19).
} 
medium by the authorities led to the foundation of television stations in every province and major city (Redl et al., 2002, 19). At the same time, television sets were made available to the public at subsidised prices: from 1979 to 1982 alone, the number of households with TV sets rose from 4.85 million to 27.61 million (Kramer, 2004, 112).

At the time, television content was largely dominated and dictated by China Central Television (Zhongguo zhongyang dianshitai 中国中央电视台; or CCTV; Lull, 1991, 24-26). It was not long, however, until local stations were increasingly able to produce their own programmes while slowly dissociating themselves from the centralistic organization (Zhang et al., 2005, 36-37). Their success has caused even CCTV to imitate their programming strategy: since the late 1980s, the central broadcaster has begun to focus more on the demands of its viewers and much less on its traditional function of offering ideological education to the masses (Kramer, 2004, 133-134). This has lead to an increase in content that is often considered "vulgar" by the authorities (television dramas, sit-coms, game shows), but is nonetheless tolerated in the name of market development and social stability: as audiences are occupied with human-interest stories, cathartically emotional dramas, and patriotic demonstrations of China's cultural superiority, their need to demand structural political changes, is believed to be assuaged (Zhao et al., 2005).

While China's television industry diversified, the authorities both at the central and the provincial level increased the population's access to TV. Chinese institutions started investing heavily in a new technology: satellite broadcasting (Harrison, 2002). The potential of reaching even the remote regions of China spurred two major endeavours: first the Beijing-based ChinaSat project, later the Shanghai-based SinoSat venture (ibid., 171-172). Satellite technology promised to function as a "new delivery weapon" that could help broadcasters "regain their political importance and generate additional revenues" (Redl et al., $2002,20)$. However, the central authorities quickly realised the ambivalent nature of their 
new projects (Zhao, 1998, 172-174). Outfitting Chinese households with satellite dishes not only enabled viewers to receive CCTV programming, but also opened the door to potentially subversive broadcasting signals from abroad (ibid.), or as Anke Redl and Rowan Simons put it $(2002,20)$ :

A combination of fears about foreign channels carried on the same satellites and the opportunities offered by alternative technologies led to the development of new policies, partly designed to stop proliferation of satellite dishes in non-remote regions. The new drive was towards investment into new communications infrastructure - cable. The process was already in full swing when satellite was unceremoniously dumped with the 1994 ban on private urban ownership of dishes.

Since this ban, the reception of foreign satellite services is permitted only "in hotels rated at three stars and above, residences approved for foreigners and certain approved organizations"; all illegal access is punishable by fines or jail terms (Harrison, 2002, 173).

Whereas these developments have caused cable transmission to become the primary broadcasting method for Chinese television, a new reform project is already taking shape. The SARFT is currently planning to transform the analogue Chinese television system into a digital one: with a combination of satellite and broad-band technology, the institution plans to fully digitalise the country by 2015 , modernizing first the Eastern regions of China, then the central provinces, and finally the West (Zhang et al., 2005, 37). If successful, the reform could increase the number of channels available to viewers from currently about fifty to over 500 (ibid.).

The rapid expansion of broadcasting capacities in China has led to a high demand for TV content (Hong, 2002, 38). Television programmes range from distinctly Chinese productions to adaptations of foreign formats or dubbed foreign imports (BBC, 2004b; BBC, 2006c; Hong, 1998, 57-76; Keane, 2002a; Keane 2005). What can be shown, and how much of the content may be foreign, is subject to strict regulation (Hong, 1998, 103; Hong, 2002, 38-39; Zhang et al., $2005,38)$. Most commercially successful television stations to some extent produce their own 
content, i.e. news, films, shows, and drama series (Kramer, 2004, 129). A good example of this is Shanghai Television (STV) which purchases large parts of its content from the Shanghai Film Studio, one of the three largest content producers in China (ibid.). ${ }^{4}$

While content production was firmly in the hands of governmentally owned enterprises until 2001, the most recent reforms by the SARFT have allowed the involvement of private companies. First, the production of television dramas was permitted to non-state firms (Zhang et al., 2005, 37). According to Zhang Junhua, almost 2,000 organizations are currently licensed to produce or distribute television films and series, with under fifteen per cent of these being owned by the state (ibid.). This shows that, whereas at the turn of the millennium content creation was dominated by state owned enterprises, the private sector now has become the driving force behind film and drama production (ibid.). In addition, legislation from 2004 enables private businesses to purchase parts of formerly state-owned media facilities (ibid.): this now entitles private enterprises to make management decisions concerning information coverage, programme structure and human resources, and has substantially altered the face of the Chinese broadcasting system.

\subsection{Structure of the Chinese Broadcasting System}

Since the introduction of television to China, the most important TV broadcaster has been CCTV (Lull, 1991, 24-26; Zhang et al., 2005, 36). With 800 million viewers, CCTV is the most powerful media giant in China today (Zhang et al., 2005, 36). CCTV has continuously expanded over the years and now carries twelve channels (Kramer, 2004, 367-368) as well as six specialized channels5: CCTV -1 is China's "comprehensive channel" (zonghe pindno 综合频 道); it produces the national news (aired on all provincial stations at seven p.m. Beijing time) as well as information programmes and entertainment. Stefan Kramer calls this station the

\footnotetext{
4Shanghai Film Studio (Shanghai dianying zhipian chan 上海电影制斻厂) is part of the Shanghai Film and TV Group (Shanghai dianying dianshi jituan gongsi 上海电影电视集团公间). See also the Group's homepage for detailed information http://www.sfs-cn.com/ [24.01.08].

${ }^{5}$ For an updated list of CCTV channels and their programming, as well as online live-streaming offers, see http://v.cctv.com/live t/index.shtml/ [25.01.09].
} 
television equivalent of the People's Daily newspaper (Renmin Ribao 人民日报; RMRB) and argues that it functions primarily as the official propaganda mouthpiece of state and Party (ibid). The CCTV-1 programmes are complemented by a channel covering economics and society (jingji shenghuo 经济生活; CCTV-2), one concerning culture, art and religion (zongyi 综 艺; CCTV-3) and an international channel (guoji 国际; CCTV-4); these four channels are available nation-wide (ibid.). In addition, viewers with cable-access receive a sports channel (tiyu 体育; CCTV-5), a movie channel (dianying 电影; CCTV-6), a channel covering the topics of children, military, science \& technology and agriculture (shao'er junshi keji nongye 少儿、军 事、科技、农业; CCTV-7), a drama channel (dianshi ju 电视剧; CCTV-8), an English-language channel (CCTV-9), an education channel (kejiao 科教; CCTV-10), a channel showing theatre productions ( $x$ iqu 戏曲; CCTV-11) as well as a channel that focuses on legal and social issues (shehui yu fa 社会与法; CCTV-12; this channel formerly promoted the image profile of China's Western regions and used to be therefore called CCTV "West", xibu 西部; ibid.). In addition, CCTV broadcasts a channel dedicated entirely to news reports (CCTV xinwen 新闻), a special children's channel (CCTV shao'er 少儿), a music channel (CCTV yinyue 音乐), French and Spanish language channels (CCTV fayu 法语 and CCTV xiyu 西语), as well as a highdefinition channel that features programmes such as US-American shows in HD (CCTV gaoqing 高清; ibid.).

CCTV is further complemented by stations at three additional administrative levels: provincial, municipal (or prefectural) and county (Hong, 2002, 29). At the provincial level, China possesses thirty-two stations (Zhang et al., 2005, 36). At the next lower level, each large Chinese city has at least one television station at its disposal (ibid.). These stations constitute the most direct method of reaching television audiences, since the broadcasters' programmes focus directly on local everyday life issues (ibid.). The final tier consists of broadcasters at the communal level. These stations have exceptionally large numbers of potential viewers, but 
are not considered particularly influential, at least from the perspective of advertisers: the majority of these viewers belongs to the rural population and hence has a far lesser income at its disposal than the urban viewers who watch the channels of stations like STV or Beijing TV (BTV; ibid.).

Some of the stations at the provincial and municipal level have grown to become strong players in the sector, and due to their regional channels and their individual programming content they today constitute serious competitors for CCTV (Kramer, 2004, 128-131). One of the first stations that were able to successfully develop a strong market presence by establishing itself as a distinctly local TV stations was STV (ibid.; Hong, 1998, 61-62). STV, which since 2001 is part of the commercial conglomerate Shanghai Media Group (Shanghai wenguang xinwen chuanmei jituan 上海文广新闻传媒集团), currently airs seven different channels (Kramer, 2004, 369): a news channel (xinwen zonghe 新闻综合), a channel covering social issues and fashions (shenghuo shishang 生活时尚), a drama channel (dianshiju 电规剧), a satellite channel promoting Shanghai's image as an economic metropolis throughout the nation (weishi 卫视), an economics channel (caijing 财经), a sports channel (tiyu 体育) and a channel for current affairs (jishi 纪实). The example of STV has been emulated by other provincial and municipal television stations (Zhang et al., 2005, 29). Notable examples of popular and influential stations are BTV, Shenzhen TV, and the Shanghai-based station Oriental Television (OTV) which, like STV, is part of the Shanghai Media Group (Kramer, 2004, 369-371). In addition, a number of pay-TV channels are available in China, for example Shanghai Cable TV (Shanghai youxian dianshi 上海有线电视; ibid.).

With the number of broadcasters growing exponentially throughout the 1980s and 1990s, a maze of institutions was developed in order to control the sector, and counteract a number of problems that had arisen from the expansion: By the end of the 1990s, many local cadres had come to discover the television and broadcasting stations at the provincial, municipal and 
communal level as important assets for disseminating propaganda and generating financial profits (Zhang et al., 2005, 36). From the view of the central authorities, this development threatened to reduce the influence that the national government and party institutions had on these stations (ibid.). In order to deal with the new complexity of the media sector and reestablish control, two governmental institutions were formed.

In March 1998, at the First Session of the Ninth People's Congress, the Ministry of the Information Industry (Xinxi chanye bu 信息产业部; MII) was created out of the merger of three former ministries (Redl et al., 2002, 18): the Ministry of Post and Telecommunications (Youzheng tongxun bu 由阝政通讯部; MPT), the Ministry of the Electronics Industry (Dianzi gongye bu 电子工业部; MEI) and parts of the Ministry of Radio, Film and Television (Guangbo dianying dianshi bu广播电影电视部; MRFT). This ministry "has been given the task of overseeing the management of Chinese information networks into the twenty-first century. The MII also coordinates state policy on construction and management of electronic media as voice, video and data technologies converge" (ibid.). ${ }^{6}$

The second relevant state institution is the SARFT, which officially has the same status and authority as a ministry (Zhang et al., 2005, 31). In 1998, it was constructed out of the remnants of the MRFT that had not been integrated into the newly founded MII (ibid.). The function of the SARFT is to "censor content and to manage the country's existing broadcast infrastructure" (ibid.; author's translation). It passes regulations concerning cable content which "are heavily focussed on the national peace, prosperity and interests, as well as disobeying national laws" (Harrison, 2002, 170). It was the SARFT that was in charge of reregulating the broadcasting industry in 1999: all television stations were pooled at the central and provincial level, and many municipal and communal stations had to merge with

\footnotetext{
- For a detailed mission statement of the ministry and an overview of its different units see the MII homepage (MII, 2005).
} 
institutions at a higher level (Zhang et al., 2005, 36). In general, the SARFT now functions as the principle regulatory authority for the film and television industry (ibid.).

In theory, the MII and the SARFT have distinct fields of responsibility. As Marc Harrison explains, "MII manages planning, administration and technical standards of broadcasting and telecommunications, which covers television, while SARFT regulates content, CCIV and the development of the cable system (...)"(Harrison, 2002, 170). In practice, however, the MII and SARFT have been fighting a turf war since their foundation (Redl et al., 2002, 19):

SARFT is anxious not to cede any further power to the superministry, while the MII is aiming at controlling all information networks including those of the broadcast industry with its cable holdings.

Considering the additional editorial involvement of other institutions such as the Ministry of Culture (Wenhua bu 文化部; MC), the State Press and Publication Administration (Guojia xinwen chuban shu 国家新闻出版署; SPPA), and propaganda departments within each broadcasting and television station, jurisdictions in the sector remain highly obscure (ibid.).

\section{2.ill The Broadcasting Market and its Primary Product: the TV Drama Series}

Due to the continuous liberalisation of China's market since the start of the open-door policy and the introduction of relaxed guidelines concerning television advertisement, the Chinese broadcasting industry has become a major source of revenues: as Zhang Junhua shows, television station profits rose from 0.98 billion RMB in 1982 to 69.6 billion RMB in 2003; a more than seventy-fold increase (Zhang et al., 2005, 35). Almost half of this sum consisted of advertisement revenues (ibid.; for an overview of advertisement increases in CCTV's budget see Zhao, 1998, 57-58 as well as Zhao, 1999, 298). Other authors also attest to the fact that advertisement has become the crucial source of income for television broadcasters (Redl et al., 2002, 22). This development constitutes a major shift away from previous financing methods: While all broadcasters were originally funded through state subsidies, "the vast majority of TV stations in urban centres are now relying totally on advertising revenue and third-party 
investment to fund their development" (Redl et al., 2002, 21). Advertising is generally restricted by legislation - during prime time, for example, the advertisement limit is nine minutes per hour - but local stations continuously attempt to circumvent these restrictions in order to make more profits (Zhang et al., 2005, 37).

One of the formats that create the highest advertisement revenues on Chinese television is the television drama series (dianshiju 电视剧; Keane, 2002b, 132). It is the most popular television format in China: according to the audience measurement company CSM, the format of the TV drama series consistently scored the highest viewer ratings during the years 2004 to 2006 out of fifteen television programming categories (between 34.6 and 36.4 per cent nationwide; the second most popular format was the news, which achieved between 12.7 and 14.0 per cent viewer ratings nationwide during the same period of time; Wang, 2007, 229). According to another survey conducted by CSM in 2004, trading of drama programmes generated revenues of 4.3 billion RMB from advertisements and programme trade that year (CSM, 2005), making it a highly profitable cultural industry. Such developments have led researchers such as Professor Hong Yin from the Qinghua University in Beijing to conclude that "television drama has gradually taken the place of cinema and become the most popular audio-visual narrative form" (Hong, 2002, 30).

The question then is what types of television dramas Chinese viewer find popular. Chinese authors' classifications of Chinese television drama genres vary widely. For instance, the authors Liu Yuan (刘园) and Dong Mei (董倜) differentiate between ten genres (Xu et al., 2006, 180-193); five 'realistic drama' genres (xianshi ticai dianshiju 现实题材电规剧) and the five 'costume drama' genres (guzhuang ticai dianshiju 古装题材电视剧). The 'realistic' dramas are (ibid.): 
1. The "main melody" drama (zhuxuanlü ju 夆旋律剧),

2. The teen drama (qingnian ouxiang ju 青年偶像剧),

3. The crime drama (po'an ju 破案剧),

4. The family drama (jiating lunli ju 家庭伦理剧), and

5. The biographical drama (zhuanjilei dianshiju 传记类电视剧).

'Costume dramas' include (ibid.):

1. The historical drama (lishi ju 历史剧),

2. The romance drama (yanqing ju 言情剧),

3. The swordsmen drama (wuxia ju 武侠剧),

4. The comedy ( $x i$ ju 喜剧), and

5. The fairy tale (shenhua ju 神话剧).

The audience measurement company AGB Nielsen, on the other hand, differentiates between twenty genres $(\mathrm{Li}, 2007,176)$ without offering precise criteria for the distinction. For instance, the company includes a range of historical genres in its listing, such as 'revolutionary history dramas' (geming lishi ju 革命历史剧), 'palace dramas' (gongting ju 宫廷 剧), 'significant historical dramas' (zhongda lishi ju 重大历史剧), 'normal historical dramas' (yiban lishi ju一般历史剧), and 'other historical dramas' (qita lishi ju 其他历史剧; ibid.). This categorization also includes criteria that are not related to content, for instance introducing genres such as 'imported drama' (yinjin ju 引进剧) and 'collaborately produced drama' (hepai $j u$ 合拍剧). Other sources refer to yet different genres, such as 'kongfu' (wuda pian 武打片), 'reworked legends' (chuanqi 传奇), or 'pink dramas' (fenhong ju 粉红剧; i.e. series that focus on the relationship problems of young, urban women and often copy the concept of US programmes such as "Sex and the City"; Keane, 2005, 84 \& 88).

The inconsistent range of genre categories makes it difficult to properly assess what drama genres audiences watch, but from the statistics provided both by AGB Nielsen (Li, 2007, 177) 
and CSM (Wang, 2007) it can be said that crime dramas, historical dramas and series that are set in an urban environment (teen dramas and family dramas) frequently attract large audiences. The question remains how those involved in the actual creation of TV drama series classify different kinds of dramas, and how such classifications and viewer preferences for certain genres influence the criteria adopted to create drama content. This problem shall be examined in more detail in Chapter 3.4.

What is important to note here is that Chinese drama series are conceived and aired differently than comparable Western formats. Other authors have emphasised the similarities that Chinese television dramas share with Western drama formats (Zhu, 2005), but these similarities pertain to filming techniques and the general conventions of character as well as plot development; my research suggests that Chinese drama series are created and structured differently from Western programmes, and that Chinese media experts believe the differences between Chinese and Western formats to outweigh the similarities. I will return to this issue later (section 3.4.iii), but first it is useful to summarise what the unique features of Chinese drama series are. Examples for this are on one hand the drama series analysed in this research project (GAJZ, HWDD, and NCNM; sequence protocols are provided on the appendix DVD), on the other hand a sample television schedule (Figure 1), which shows a section from the evening programming of CCTV- 1 and CCTV-8 on a randomly chosen day in 2005.7

\footnotetext{
${ }^{7}$ Source: http://www. liaohe.net.cn/tv_programme/CCTV-1.html [03.01.08]; author's translation.
} 
Figure 1: Sample TV Schedule, Evening Programme for 1 February 2005 (Tuesday)

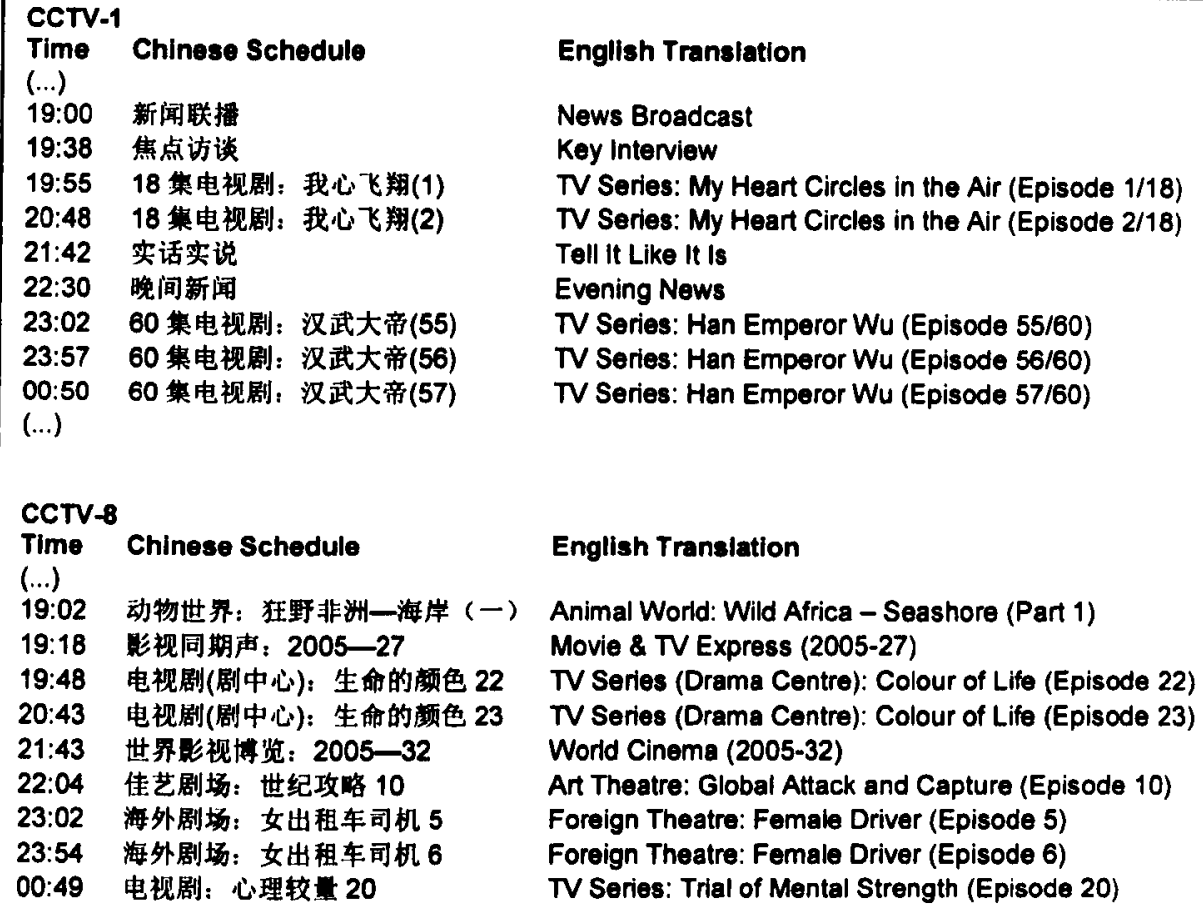

\section{English Translation}

\section{News Broadcast}

Key Interview

TV Series: My Heart Circles in the Air (Episode 1/18)

TV Series: My Heart Circles in the Air (Episode 2/18)

Toll it Like it is

Evening News

TV Series: Han Emperor Wu (Episode 55/60)

IV Series: Han Emperor Wu (Episode 56/60)

TV Series: Han Emperor Wu (Episode 57/60)

\section{English Translation}

Animal World: Wild Africa - Seashore (Part 1)

Movie \& TV Express (2005-27)

TV Series (Drama Centre): Colour of Life (Episode 22)

TV Series (Drama Centre): Colour of Life (Episode 23) World Cinema (2005-32)

Art Theatre: Global Attack and Capture (Episode 10)

Foreign Theatre: Female Driver (Episode 5)

Foreign Theatre: Female Driver (Episode 6)

TV Series: Trial of Mental Strength (Episode 20)

First, a series of peculiarities can be discerned concerning the structure of the broadcasting schedule:

1. On both stations, television dramas are the predominant television format during the evening hours. This observation is also made by Stefan Kramer $(2004,361)$, and is further exemplified by the average annual per centage of broadcasting time allocated to TV drama series on China's TV channels, which amounts to roughly twenty-five per cent (Wang, 2007, 229).

2. For each series, two or three episodes are shown consecutively (note how three episodes of HWDD are aired in a row in Figure 1).

3. The time table is not structured by the hour like most Western television schedules are: a new programme starts when the previous one has ended, regardless of the time (note the arbitrary starting times, particularly of the television dramas, in Figure 1). 
4. Television dramas episodes are not broadcasted at a weekly interval, but are instead aired consecutively, two to five episodes per day, until the drama is concluded. ${ }^{8}$

Second, there are a number of distinct characteristic that television drama series share with regards to the way their plot is structured and presented:

1. Chinese drama series are conceived as closed, over-arching narrations, which are usually told within twenty episodes. This sets them apart from continuous 'open' serials such as the Western 'soap opera' format, and places them more in the vicinity of the 'closed' Latin-American telenovelas (Allen, 1995, 17-24).

2. In China, 'sequels' or different 'seasons' of a TV drama series are not usually produced: If a series is followed by a sequel, the new programme will only retain the name and general theme of the original, but will feature an entirely new storyline, new characters, and new actors. Examples of this are the three instalments of GAJZ as well as the various spin-offs of NCNM, which do not continue the narrative of previous instalments but constitute closed narratives in their own right.

3. Chinese television dramas do not possess as clearly a defined episode structure as most comparable Western programmes, i.e. the various narrative arcs are not created to span across precisely one episode, ending in a cliff-hanger ending at the end of each episode (see the three sequence protocols on the appendix DVD for proof of this observation). This has the effect that when watched on DVD, Chinese drama programmes seem more like a sixteen-hour drama movie than a television series. Even though the programme is later broadcasted in episodes that have the standard length of roughly fourty-two minutes, episodes will usually start with the last shots of the previous episode, and then move on to the subsequent scene, essentially picking up the narration exactly where the programme previously left off (for

\footnotetext{
This peculiarity is not demonstrated in Figure 1 , but can be observed in the TV schedule of CCTV as provided on the homepage www.cctv.com/tvguide/index.shtml [27.07.08].
} 
examples see the mid-scene transition in GAJZ from episode 12 to 13 or in NCNM from episode 4 to 5 , as well as the break between two narratives in HWDD in the middle of episode 14).

4. The starting and ending credits of each episode of Chinese television dramas are constructed in a way that provides a montage of scenes from the series in its entirety, and it is not uncommon that key scenes or a drama's conclusion are already shown in the opening sequence, or later during the end credits of a television drama, essentially 'giving away' major plot points that have not yet been shown (for example, the opening credits of NCNM conclude with a scene from the final episode, showing the main characters getting married). This suggests that the concept of suspense (i.e. of not knowing the outcome of a narrative) that most Western viewers are used to from programmes such as 24, Lost or Prison Break is not a defining aspect of television dramas in China.

I shall return to these observations later in Sections 3.4.iii as well as 3.7, when discussing the viewing habits that Chinese media experts believe characterise the Chinese audience, as well as the functions that television drama is said to have in China.

\subsection{The Chinese TV Drama Audience}

Considering the market value of Chinese television drama series and the importance that advertisement revenues have for the TV stations, the question for producers and broadcasters alike is: who watches television dramas in China? As Anke Redl and Rowan Simons point out, the lack of so-called "accountable audience measurement tools" is becoming a major obstacle for generating profits in the industry (Redl et al., 2002, 22). To date, reliable data on audience ratings comparable to that used in most industrialised countries is still rare in China (ibid.). Current audience research in China mainly relies on the data produced by two institutions: The first is CVSC Sofres Media Ltd, or CSM (Yangshi-Suofurui 
meijie yanjiu youxian gongsi 央视-索福瑞媒介研究有限公司). ${ }^{9}$ CSM is a joint-venture between the French media research company Taylor Nelson Sofres (TNS) and the Chinese enterprise CTR Market Research. 10 The second relevant company is the China branch office of AGB

Nielsen Media (formerly known as AC Nielsen Media)."1 The work of these two institutions differs substantially, and the statistical data they produce is not congruent. The main reason for this is that the two institutions base their calculations on different samples: CSM measures the viewing habits of 178,800 panellists in 54,000 households across China (covering, as the company profile both on the TNS and the CSM homepage states, "China's 1.2 billion TV population"); this includes viewers both from rural and urban China, as well as from the Special Administrative Region of Hong Kong. ${ }^{12}$ In contrast, AGB Nielsen covers mainly urban China; it used a sample base of over 11,000 panel households in late 2007, which according to company information represented " 471 million Chinese TV viewers". ${ }^{13}$ What problems arise for producers and broadcasters from the use of the data provided by these companies will be discussed in Section 3.4.i. For now, it is worth turning to the question of who the typical viewers of Chinese TV drama series are according to the

\footnotetext{
${ }^{9}$ A detailed presentation of the company in English as well as an online rating service for television programmes, TV drama series and TV sporting events in China's largest urban centres since 2004 is presented on the CSM homepage http:/hww.csm.com.cn [20.11.07].

${ }^{10}$ For more information on TNS, see the company homepage http://www.tnsglobal.com/ [20.11.07]. An overview of CTR Market research can be found on the wobpage http://www.ctrchina.cn/en/about/index.html [20.11.07]. The information provided there shows that CTR is itself a joint venture between TNS and the China International Television Corporation (CITVC), which is in turn "the sole wholly-owned subsidiary of China Central Television (CCTV)" (ibid.) This makes CSM indirectly subordinated to CCTV.

${ }_{11}$ For information on AGB Nielsen see the webpage http:/WwW.agbnielsen.neVwhereweare/dynPage.asp?lang=english id=420\&.country=China [20.11.07]. A third institution that produces ratings data is the Horizon Research Consultancy Group (Lingdian diaocha gongsi 零点 调查公司); however this enterprise primarily focuses on market research and market entry solutions, as their company homepage shows (http://www.horizon-china.com/servlet/Page?Node=8724 [20.11.07]). It does not play as important a role in the TV ratings measurement sector as CSM or AGB Nielsen.

${ }^{12}$ See both the TNS homepage http://www.tnsglobal.com/news/newsE8768F249EA94150822C9510AF5B3861.aspx [20.11.07] and the CSM website on China and Hong Kong http://Www.csm.com.cn/en/about/indexhk.html [20.11.07].

${ }^{13}$ According to the information provided by the company on its internet homepage

(http://www.agbnielsen.net/whereweare/dynPage. asp?father=420\&lang=english\&id=303\&country=China [20.11.07]), the research sample includes households from the municipalities of Shanghai, Beijing. Chongqing and Tianjin, as well as from the provinces of Guangdong. Zhejlang. Jiangsu, Shandong, Fujian, Sichuan and Liaoning. AGB Nielsen plans to include all of mainland China in its calculations in the future. It has announced that it will presently widen its sample base to "16 provinces, based on 18,000 panel households" (ibid.). AGB Nielsen does not specify which 16 provinces it plans to include, but considering that the company's medium-term aim is to provide "overnight TV ratings for nearly 900 million Chinese, or in other words, nearly two thirds of the Chinese population who contribute more than $80 \%$ of the national GDP" (ibid.), it seems safe to assume that the urban population will continue to constitute the main sampling share in the near future.
} 
available statistical data, and how these viewers react to the current offer of drama entertainment on Chinese TV. Answers to these questions are provided by the CUC researchers in Beijing who have analysed the data provided by AGB Nielsen on how many minutes per day urban Chinese viewers watched television drama series in the year 2005, as well as what feedback viewers provided to TV stations concerning the programmes that had been aired ( $\mathrm{Li}, 2007) .^{14}$

According to the assessment of the CUC researchers, the appeal of television drama series depends on the following five criteria:

1. Gender: Drama viewers are predominantly female (with women watching roughly eight minutes more television drama per day than men; ibid., 184).

2. Age: The main age group that watches television dramas are viewers older than 45 (with the age group 45-54 on average watching 72 minutes of drama per day, and the age group above 55 years watching 84 minutes, i.e. roughly two episodes per day; ibid.). Young viewers generally watch less television drama, and the age group of the 25-34 year-olds has the lowest average viewing time, watching only about half the amount that the oldest audience group consumes (44 minutes per day; ibid.).

3. Education: According to the survey, audiences with an elementary or middle-school diploma watch the most television drama (between 60 and 70 minutes per day; ibid.), whereas university graduates watch the least amount (48 minutes per day; ibid).

4. Prosperity: A similar decrease in viewing time seems to accompany rising incomes: the group classified as "low monthly income" in the survey (less than 1,000 RMB per month) watched roughly 62 minutes of television drama per day, compared to only $\mathbf{4 8}$ minutes per day for the group with "high monthly income" (more than 2,000 RMB per month; ibid).

5. Place of Residence: The data presented by the CUC researchers suggests that TV dramas equally constitute the largest share of television consumption for viewers in all metropolises across the nation, generally amounting to just above $\mathbf{4 0}$ per cent (Beijing 39 per cent, Chongqing 46 per cent, Shanghai 42 per cent, and Guangzhou 43 per cent; ibid., 185-198). There are, however, differences in the degrees

\footnotetext{
${ }^{14}$ It should be pointed out that the date provided by AGB Nielsen does not include rural viewers, and hence cannot represent all of China. How this problem affects the way in which producers and broadcasters target their programmes at certain viewers is discussed in Sections 3.4.1 and 3.4.il.
} 
to which viewers from different regions consume dramas aired on CCTV. For instance, CCIV drama consumption is noted as highest in Beijing (24 per cent compared to dramas aired on local or satellite stations), followed by Chongqing (19 per cent). In contrast, viewers in Shanghai only watched CCTV drama broadcasts 11 per cent of the time, and audiences in Guangzhou hardly watched any CCTV series at all (4 per cent; ibid). If these numbers are to be believed, CCTV dramas are generally more likely to be watched by northern or western Chinese audiences than by viewers living along the southern and eastern coastal regions. It is likely that this difference in CCTV consumption arises from alternative offers from other broadcasters, such as STV in Shanghai (Kramer, 2004, 130) and various Hong Kong stations that are received in Guangzhou (Fung et al., 2002).

The questions of whether those involved in the actual production and broadcasting of TV dramas share this assessment, and how their perception of target audiences influences their work, are discussed in section 3.4.ii. Let me turn to the second issue that the CUC research project addresses, i.e. what kind of feedback Chinese TV drama viewers provide to the TV stations that broadcast the programmes. ${ }^{15}$ I have summarised the top eight issues that viewers raised in 2005 according to the researchers in Figure 2 (Li, 2007, 226-227; author's translation).

In addition to analyzing the general topics that viewers commented on as well as the form in which these comments were made (letters, phone calls, email, mobile phone text messages and message board posts; ibid., 225), the CUC researchers also provide sample remarks from the letters and emails sent to the respective stations (ibid., 229-231). These statements are part of the analysis provided in Section 3.4.v of this thesis.

\footnotetext{
${ }^{15}$ The researchers conducted interviews with employees from twelve different broadcasting institutions (CCTV, Beijing TV, Shaanxi TV, Shanxi TV, Sichuan TV. Chongqing TV. Anhui TV. Hubei TV. Hunan TV, the Shanghai Wenguan Group, Zhejiang TV and Guangdong TV). In addition to the interviews, viewers' feedback letters to CCTV, Beijing TV, and Anhui TV were evaluated, as where the emails sent to BTV (between September and December 2005) and the message board entries made on the websites of Shanxi TV and Zhejiang TV; for all other stations, the feedback files had either been lost or not yet processed $(\mathrm{Li}, 2007,225)$.
} 
Figure 2: Main Concerns of Viewer Feedback (Ordered by Quantity)

\section{Rank Feedback losue}

1. Demand that a previously broadcasted television drama be repeated. (要求重播电规剧)

2. Suggest a drama for broadcasting; for instance a programme that was aired on another station or was mentioned in a magazine.

(点播电视剧)

3. Inquire about a television drama; for instance inquire when a programme will be aired, where the DVD can be purchased, how the end title song might be obtained, etc.

(冾询电视剧情况)

4. Comment on a television drama; i.e. voice an opinion concerning content, acting, etc. (对电视剧的评价)

5. Ask that a television drama's broadcasting time be changed.

(调整电视剧播出时间)

6. Complain that a TV drama was cancelled midway.

(投诉电视剧中途停播)

7. Complain that a TV drama was announced to not be broadcast.

(投诉顶告电视剧未播放)

8. Complain about too much advertising.

(投诉广告太多)

\subsection{The Debate: TV Content between Market Liberalisation and State Control}

The numerous reform strategies that have been applied to China's media market in recent years (Zhang et al., 2005, 36-38) as well as the popularity of TV drama entertainment has sparked numerous debates, both in and outside of China. In the PRC, the popularity of drama shows has led domestic critics to question the cultural, political, social and aesthetical value of this particular entertainment format (Hong, 2002, 33). According to Hong Yin, many intellectuals take a "militant 'anti-marketization' stance", criticizing television drama's supposed "lack of social responsibility, its audiences, its social function, the problem of cultural globalization, and its tendencies towards vulgarity", often demanding stricter government controls of televised content (ibid., 33). At the same time, popular television is not without its supporters among Chinese intellectuals (ibid.): 
There are also, however, intellectuals who embrace the common values and popularization of culture brought about by commercialization. They argue that this kind of change has allowed television drama to break away from its pedagogic and propagandist tradition, and also from its elitist aristocratic tradition. And while people ought to be vigilant about vulgarity, commercialization is indeed a revolution of some significance, one that has brought an affective element to drama.

What role these stances play in the production of television drama content will be examined in Chapters 3.6 and 3.7.

Outside of China, the debate has concerned the question of how market liberalisation has affected the broadcasting system. The liberalisation strategies have allowed television stations to increasingly adopt market orientated management and private ownership structures (Kramer, 2004, 128-131). Many stations, for example STV, today employ a staff of professional television experts and adhere to a pragmatic set of quality criteria modelled after those of Hong Kong television stations (ibid.). In many cases, the ownership structure has also been subject to reform (Zhang et al., 2005, 29; Zhao, 1998, 112-114). In addition, the reforms have made the dissemination of technological innovation possible across the country (such as broad-band internet access and digital television), and have also enabled foreign firms to engage in joint-venture-projects and the extensive trading of cultural products (Zhang et al., 2005, 36-38 and 42-43). The soaring growth rates in the cultural sector (approximately ten per cent in 2001; ibid., 41) along with the increasing involvement of global players such as News Corporation, Viacom and Sony Pictures (Feuilherade, 2005) have prompted international observers to speak about a "breakneck media revolution" in the PRC (Griffiths, 2005).

Yet, at the same time, the optimistic assessments of recent Chinese media developments are offset by regular reports of extensive market regulations for foreign firms (BBC, 2004a) as well as of content censorship on television (BBC, 2006b), in the press (Holbig, 2006), and on the internet (Holbig, 2005; BBC, 2006a). The contradiction between market reforms and 
continuous state control has led to a debate concerning the question of how open the newly reformed media market in China truly is. Authors such as Stefan Kramer argue that even though Chinese television broadcasting has become a strongly commercially driven enterprise, it is nevertheless the primary tool for propaganda work (Kramer, 2004, 464-465). According to Kramer, the illusion of direct and unaltered coverage of the truth that television provides makes it the central mouthpiece for the CCP and the state (ibid.). The argument is that important events are first edited and tailored to represent the official viewpoint of the nation's leadership, then aired by CCTV at prime time, and next disseminated throughout the country via the provincial television stations; the reality portrayed on television, so the argument goes, is finally flanked and strengthened by follow-up articles in the state-owned print media such as the RMRB (ibid.). Other authors, such as Zhang Junhua, share this assessment of the situation in China, arguing that television has become the main anchor for the authority's ideological work ever since incomes have risen enough to enable the majority of the population to own a television set (Zhang et al., 2005, 36; author's translation):

As long as standards of living were relatively low, newspapers and cinemas offered the best way of propagating political information. Meanwhile, television has moved to the centre and constitutes the last bastion of the propaganda machinery.

The importance that the authorities attribute to television content as a propaganda tool is reflected in the array of regulations and censorship proceedings that govern broadcasting, both at the news and the entertainment level. According to the Chinese media scientist Hong Yin, CCTV and provincial stations are required to broadcast only television drama series during prime time that conform to the propaganda guidelines of the party and state (socalled 'socialist main melody' dramas (shehui zhuyi zhuxuanlul 社会主义主旋律; Hong, 2002， 33). In addition, the stations are required to "reduce the amount of popular entertainmentoriented 'Royal Court' costume plays and kung fu stories" that are aired during this time (ibid.). Furthermore, the authorities operate an intricate management system with the 
intention of strengthening the CCP's supervision of content production. Citing the example of Chinese television drama production, Hong Yin describes the four levels of this system as follows (Hong, 2002, 36):

The first is that a television drama production permit system was instituted on 1 June 1986. Any organization wanting to produce drama had to gain a permit from the Ministry of Film Radio and Television (later SARFT). (...) The second area of strengthening management concerns lies in the actual policing of regulations. Before production can begin, the production unit must report the topic to the Chinese Television Arts Committee for clearance. These regulatory bureaux decide what may proceed at any given time according to the current political terrain and certain approved drama topics. The third area is distribution supervision; (...) the distribution and broadcast of television drama has to navigate the supervision of local CCP propaganda departments. Fourth broadcasting itself is monitored. Today SARFT, CCTV and provinciallevel stations have television inspection procedures. When programmes exhibit 'political problems' they can be officially terminated. These measures ensure that production and circulation of programming accords with the political interests of the state.

At first sight, such procedures make the Chinese media market appear to be highly regulated and strongly censored by the authorities. The question of what role censorship (shencha 审查; literally: 'examination' or 'investigation') plays in the system is, in a sense, dependent on what is understood as censorship. The term itself can be defined either narrowly, or very broadly. A narrow definition is provided by The Oxford World Encyclopaedia, which refers to censorship as a "system whereby a government-appointed body or official claims the right to protect the public interest by influencing the release of any item of mass communication," and states that censorship "falls into four broad categories - politics, religion, pornography or violence" (Censorship, 2005). Such a definition essentially sees censorship as the prohibition of certain content by government institutions. However, many authors question whether such prohibitions still truly function in a liberalised market such as Chinas. As Donald and Keane argue, "centralized control of the media does not necessarily translate into compliance by producers with chapter and verse of the regulatory canon. The media in China are heavily regulated in theory but, due to the sheer size of the media sector, 
guidelines and policies are difficult to implement and enforce" (Donald et al., 2002a, 7). Hong also concedes that the state supervision of television drama production is continuously adapting to the logics of free market development: “(...) non-television companies can produce television drama, accept non-state funding, accept investment from foreign and overseas enterprises as well as joint funding arrangements, and allow regional executive branches to monitor without incurring centralized censorship inspection. These relatively relaxed administrative controls make the production of television drama appear a more pluralistic undertaking" than that of cinematic movie production (Hong, 2002, 36). According to Zhang Junhua, such changes have been brought about by China's accession to the WTO in late 2001: As China 'entered the world' (Zhongguo ru shi 中国入世), a shift in cultural policy took place (Zhang et al., 2005, 30) that caused governmental authorities to no longer function as creators and carriers of culture (ban wenhua 办文化) but rather as administrators (guan wenhua 管文化). Official institutions such as the SARFT or the MII, so the argument goes, increasingly see themselves as providing the general framework for media production and broadcasting while rarely exerting direct control (ibid).

However, analyses of the Chinese media by researchers such as Guo Zhenzhi (Zhao et al., 2005), Barrett McCormick (2004), Zhao Bin (1999), or Zhao Yuezhi (1998) show that just because centralised, administrative prohibitions have become less important in China, this does not mean that the system is not subjected to various sorts of softer censorship, such as self-censorship or government incentives for the production of certain content. In order to account for such mechanisms, a broader definition of the term censorship is required, such as the one provided by Robert E. Drechsel (2005), who describes censorship as "any restriction on the dissemination or content of expression (...)". How various 'hard' and 'soft' mechanisms influence the creation and dissemination of television drama content in China shall be analysed in Chapter 3.5. 
What is evident from the research described above is that the administrative controls that govern the Chinese broadcasting system have changed, and this has led researchers to argue that the propaganda messages of prime-time central television have also shifted (Donald $e t$ al., 2002a, 14):

Rather than broadcasting doctrinal propaganda about the truth of socialism and the decadence of capitalism as in the past, the Chinese media now inculcate lessons about what it means to live in a changing world where competitiveness drives national economic productivity.

The development of the Chinese broadcasting industry shows, as observers argue, the tradeoff with which the authorities are currently confronted in China: On the one hand, deregulated markets create profits and rising living standards; on the other hand, an excessively liberalised media system ceases to function as an effective propaganda tool and eventually threatens to completely elude management by the authorities (Donald et al., 2002b, 200).

What is remarkable in this context is the argument by Mark Harrison from the University of Westminster in London. Harrison states that despite the degree to which the Chinese broadcasting system has been liberalised, television content in China has not become significantly more diverse (Harrison, 2002, 176). Instead, so Harrison observes, Chinese television displays a "surprising uniformity" of programming content (ibid.). Harrison argues that types of Chinese television entertainment are not varied at all, but in fact converge, reproducing "the historically-specific and evolving ideologies of what constitutes the experience of being nationally Chinese. Therefore, even the very large urban centres of Shanghai and Guangzhou do not produce a sufficient amount of local and regional programming to compete with the political and cultural hegemony that nationalist ideology exercises over the rest of China" (Harrison, 2002, 177). Such an assessment stands in stark contrast to the argument made by Stefan Kramer that Chinese audiences are increasingly 
assembling counter-hegemonic discourses from what they are presented with on TV (Kramer, 2004; 2006).

The current debates on the situation in the Chinese broadcasting sector and the relevance of television drama series in China raise a number of questions that have not been sufficiently addressed by past research. It is not clear from previous accounts:

1. How the tension between market liberalisations and state controls actually affects the production and broadcasting of television content;

2. To what degree the Chinese authorities are truly influencing the production and broadcasting of such content; and

3. What kinds of political messages are disseminated under the present conditions.

By examining the factors that lead to the production of television drama series in China, as well as the political content of three such drama series, the analysis provided in Chapters 3 and 4 will shed light on these issues.

\subsection{The Theory of Discourse}

The theoretical orientation of this research project is derived from a discourse analytical approach. In this sense, the present analysis has a similar theoretical foundation as some of the studies mentioned above (for instance Rofel, 1995; Geraghty, 1995). However, it differs substantially in its methodology, specifically in the way it links the tools of discursive fineanalysis to the methodology of visual communication analysis. But before turning to these issues, it is necessary to define what the term 'discourse' refers to in this dissertation.

\subsection{Communication Practices and the Construction of Truth}

For the purpose of this research project, I define discourse as communication practices that systematically construct our knowledge of reality, and consequently both the objects and the subjects of communication (for a full glossary of terms and their definitions see Figure 8 in Chapter 2.7). The author who is arguably most associated with such an interpretation of the 
term discourse is the French philosopher and sociologist Michel Foucault. The aim of Foucault's research was to analyze "regimes of truth" (Potter, 2005, 86) and their history, i.e. the development, demise and reoccurrence of statements. His main question was: what could be said in what way at specific points in time? As Foucault himself wrote in his early work (Foucault, 2005, 30-31):

We must grasp the statement in the exact specificity of its occurrence; determine its conditions of existence, fix at least its limits, establish its correlations with other statements that may be connected with it, and show what other forms of statements it excludes. (...) The question proper to such an analysis might be formulated in this way: what is this specific existence that emerges from what is said and nowhere else?

Foucault originally attempted to map the rules that govern the emergence of such statements, as well as the historical ordering of knowledge (Dreyfus et al., 1982, 102-103). This "archaeological" approach, which Foucault applied to such fields as the history of medicine, of psychology, and of the social sciences (Foucault, 2005b), questioned the objectivist position on truth. It aimed to uncover mechanism such as classification strategies or theory building that Foucault argued created the objects of our social world (Dreyfus et al., 1982, 61). Due to the assumption that such practices were based on overarching regularities, authors such as Dreyfus and Rabinow identify Foucault's early work with the structuralist paradigm of that period and argue that Foucault's early conception of discourse was caught in the same ontological dilemmas that he had originally set out to solve (Dreyfus et al., 1982, 16-17). It was not until Foucault's later analyses of power relations that he was able to leave these problems behind. Foucault's works on disciplinary and confessional technologies (Foucault, 1990 \& 1995) focus no longer solely on the "objects" of discourse but instead bring the "subjects" back to the fore: In these later "genealogical" works, Foucault explicitly questions the researcher's role in the creation of knowledge, and hence of power relations; this marks a shift from the idea that historical rules govern communication to the realization that social 
practices construct (and in turn are being constructed by) discourse (Dreyfus et al., 1982, 118 -

119 \& 199). David Howarth $(2000,72)$ from the University of Essex explains:

Genealogy is thus explicitly concerned with the centrality of power and domination in the constitution of discourse, identities and institutions and involves the adoption of a critical ethos towards them (...).

Through this approach Foucault proved how specific opinions came to be formed and preserved as what is today commonly called the hegemonic discourse, that is as the dominant viewpoint(s) throughout society, kept stable by political power dynamics (MacDonald, 2003, 32).

Today, the analysis of discourses is a well-established method in the social sciences. It is, as the German discourse analyst Siegfried Jăger points out, a method that is not only capable of revealing "with what means and for what 'truths' acceptance is created within a population; what is to be considered normal and abnormal; what can be said (and done), and what cannot" (Jäger, 2004, 223; author's translation), but also an approach that "(...) offers clues to the ideological assessment concerning, for example, the fundamental understanding of society, the internalised generic conception of man, positions on new technology, ecological questions, expectations of future developments, questions of human existence, perceptions of general normality and truth, etc" (Jäger, 2004, 184; author's translation). The analysis of discourses is, therefore, not primarily concerned with hermeneutic questions such as "what is being said in what is said?" or linguistic problems such as "according to what rules has a particular statement been made, and consequently according to what rules could other similar statements be made?" (Foucault, 2005a, 30). It is instead the analysis of communication practices that systematically form the subjects and objects of which they speak (Dreyfus et al., 1982, 109 \& 203; Howarth, 2000, 82-83; Jager, 2004, 116), and this is the definition of discourse analysis that forms the basis of the present research project. In this sense, the term 'discourse' is regarded as a constructivist concept. 


\subsection{The Anglo-Saxon Tradtion of Critical Discourse Analysis}

Such a constructivist interpretation of discourse is essentially grounded in continental

European usages of the term, which often clash with the Anglo-Saxon understanding of the word. This becomes particularly clear when considering the work of researchers in the Anglo-Saxon tradition of Critical Discourse Analysis (CDA), such as Lilie Chouliaraki (Chouliaraki et al., 1999), Teun van Dijk (1993), Norman Fairclough (1995), or Myra McDonald (2003). ${ }^{16}$ Such CDA analyses certainly have their merits: for instance, the British linguist Norman Fairclough demonstrates skilfully how the use of certain analytical tools can uncover ideological positions shaped by rhetoric and linguistic devices (see in particular his analysis of liberal ideology in the British "enterprise discourse" in various political speeches and promotion texts of the 1980s; Fairclough, 1995, 112-129). However, while CDA authors such as Fairclough share the Foucauldian view that discourse reflects and reinforces power relations across society, and that this "interconnectedness of things" needs to be made "visible" (and then criticised; hence the use of the term "critical" in "Critical Discourse Analysis"; ibid., 36), they do not attribute the same constructivist power to discourse as German discourse analysts such as Jürgen Link or Siegfried Jăger do (Jäger, 2004, 111-112). Even though Fairclough argues that language is not only "socially shaped", but "also socially shaping, or constitutive", and that language use "is always simultaneously constitutive of (i) social identities, (ii) social relations and (iii) systems of knowledge and belief" (ibid., 131), he nonetheless seems to be arguing at the same time that there are different types of knowledge (ibid., 44): On the one hand "beliefs, values, ideologies" (in a sense, types of false knowledge), and on the other hand "knowledge properly so called" (what one might view as true

\footnotetext{
${ }^{10}$ It should be noted that despite the overarching simllarities of CDA approaches, there are also some differences. For instance, as Greg Philo explains in his comparison of van Dljk's and Fairclough's work (Philo, 2006), van Dijk's approach to CDA examines a wider variety of structural issues (text layout, headlines, graphic arrangements, otc.) and also places more emphasis on quantitative methods of analysis. Also, while authors such as Fairclough and Chouliaraki try to link their theoretical work to post-modem cultural critiques (Chouliaraki ef al., 1999,79 \& 89-93), van Dijk criticizes post-modemism as esoteric and tries to exclude it from his own research agenda (van Dijk, 1995). Finally, van Dijk's interpretation of the term "ideology" is purely functional and consequently stands in stark contrast to normatlve usages of the word in other CDA approaches $(2003,24)$; it is therefore much closer to the semiotic definition of ideology used in this research project (see Section 2.4.ii).
} 
knowledge). The latter includes "judgements of truth and falsity", such as required "when faced with issues such as war crimes in ex-Yugoslavia" (ibid., 19), but also inferences about the true state of the world, such as the knowledge "that there is some identifiable door which is closed when some speaker asks some addressee to 'open the door'" (ibid., 31). In this sense, Fairclough distances himself somewhat from the Foucauldian concept which understands discourse as the construction of all truth, and argues instead that there is such a thing as false truth that has been pulled over the actual truth by certain interest groups to promote their power interests; to this end he introduces the Marxist concept of ideology to his theory (signifying precisely such false truths), hoping that it will shift the focus away from the Foucauldian institutions of power and instead to the struggle of different classes who are allegedly vying for ideological dominance over society (ibid., 17; for a similar use of the term ideology in CDA see MacDonald, 2003, 36). I shall not follow the CDA interpretation of the term ideology in this project, seeing as the introduction of such a term to discourse analysis threatens to obscure the notion that all communication practices continuously construct all of our knowledge, and hence all of reality. This constructivist aspect of discourse is essential: In the interpretation used in this thesis, discourse is not merely, as some researchers put it, the "socially constructed knowledge of (some aspect of) reality" (Kress et al., 2001,4) or a system of communicative practices that "help to construct specific frameworks of thinking" (MacDonald, 2003, 1), it is not a reflection, mirror, or representation of reality, but its primary constitutive (Jäger, 2004, 23).17

Another problem with CDA approaches is that they focus mostly on written or spoken texts. As Greg Philo from the Glasgow University Media Group criticizes, CDA researchers primarily produce "text only" analyses $(2007,185)$. This becomes clear when considering

\footnotetext{
17 There has been considerable debate between constructivist (or 'relativist') analysts and objectivist (or 'realist') researchers concerning the advantages and drawbacks of each approach lo science. I do nol wish to go into these debates here. The realist point of view is presented by Sokal et al., 2003, 49-95, for a relativist position see Edwards et al., 1995. Suffice to say that the discourse analytical approach adopted in this thesis agrees with the epistemological and ontological implications of relativist theories while at the same time not condoning moral relativism.
} 
Fairclough's definition of discourse, which refers both to "language use conceived as social practice" and a "way of signifying experience from a particular perspective"; the term discourse practice then means "the production, distribution and consumption of a text", i.e. of "the written or spoken language produced in a discursive event" $(1995,135)$. Essentially, this definition narrows the term discourse down to speech acts and written documents, while ignoring other communication practices (such as communication through images or music; van Dijk, 1993, 264). Although CDA researchers point out that their framework also accounts for other forms of communication than merely language (i.e. the "design" of advertisements or the usage of images; Chouliaraki et al., 1999, 10), the interaction of these different discursive modes are not made explicit. A consequence is that in some cases researchers overlook the importance of the visual and offer interpretations of discourse that are not plausible. An example is Fairclough and Chouliaraki's analysis of a Christmas advertisement for a charity organization (ibid., 10-15). Although the researchers point out that the use of a photograph and the particular design of the ad are relevant, their analysis focuses on linguistic issues, primarily the tag-line underneath the photo, which reads: "We could try to guilt trip you" (ibid.). The author's then take this declaration, along with the written explanation beneath it, at face-value and argue that the advertisers are criticizing guilt-tripping charity advertisements produced for the Christmas holidays as a form of commercialization that the institution itself does not condone. What the researchers fail to see is that the catch-phrase and the explanatory paragraph below it work in combination with the image: a close-up, frontal photo of a young man, looking straight into the camera, with a slightly sad look in his eyes. The effect is that the advertisement indeed does "guilttrip" the reader, but attempts to justify this attempt through a rhetoric device. Instead of questioning the dynamics of this advertisement, the researchers argue that the language-use is a counter-hegemonic act of re-assembling discourse in order to contest the logic of contemporary capitalist society. 
This questionable interpretation results on one hand from the purely text-based analytical focus of CDA. On the other hand, it is grounded in an inherent bias to support the struggle of marginalized groups against the alleged dominance of capitalism and (late) modernity (ibid., 93). In the above example, the author's do not consider that the advertisement may be implicitly reinforcing power relations rather than battling them, precisely because they regard the social organization responsible for the poster as inherently "good". In this sense, CDA is more than a scientific approach, it is a political project, or as van Dijk writes, the work of critical discourse analysts "is admittedly and ultimately political" $(1993,252)$. As such, CDA makes a number of assumptions and claims that are not unproblematic. First, its supporters argue that the researcher can (and should) take a normative position on the issues he or she analyses; in other words, the discourse analyst is in a position to evaluate which discourses are good and which are bad (Chouliaraki, 1999, 4 \& 136). Second, CDA authors make a choice concerning the value system they believes should be endorsed. In the words of Chouliaraki and Fairclough (ibid., 8): "CDA is a matter of democracy in the sense that its aim is to bring into democratic control aspects of the contemporary social use of language which are currently outside democratic control...". Not only do the author's fail to specify what they mean by "democracy" (and apparently they see no contradiction between the CDA claim to critically question social control through power relations and the simultaneous attempt to bring social relations under "democratic control"; ibid.), they furthermore offer no explanation of how the CDA researcher is able to assure that his emancipatory project is not part of precisely the economic and political domination it is claiming to fight. Foucault himself criticized such humanist claims to absolute truths precisely for this reason: "the belief that one is resisting repression, whether by self-knowledge or by speaking the truth, supports domination, for it hides the real working of power" (Dreyfus et al., 1982, 169). Put in other words, the political project of CDA may well cover up and reinforce the very practices it is trying to unveil. 


\section{3.iil The Post-Marxist Approach to Dlscourse Analysis}

Aside from CDA researchers, other linguists and social scientists have also developed approaches to discourse analysis, which in some cases differ substantially from the central CDA ideas. Particularly Post-Marxist authors such as David Howarth from the University of Essex $(2000,99)$ distances themselves from the argument that discourse is defined primarily by the class struggle of late-modern capitalism. Howarth, for instance, acknowledges the contribution of Marxism as a critical social science approach, but also discusses how Marxist thought nonetheless failed to give discourse analysis a broader applicability to social phenomena due to its recourse to overarching structural determinants such as economic forces (ibid.). To counter these problems, Howarth introduces discourse interpretations by authors such as Laclau and Mouffe, which he views as the pinnacle of the discourse project: He argues that Laclau and Mouffe not only reaffirm the constructivist notion of discourse (ibid., 104), but also widen the range of materials that lend themselves to discourse analysis (ibid., 140). Methodologically speaking, the present approach therefore shares Howarth's premises (ibid.):

Discourse analysts (..) gather primary information from a range of possible sources, which include surveys of newspapers, official reports, 'unofficial' documents such as pamphlets, organizational minutes and agendas, personal biographies and media representations such as television documentaries and films. They also supplement these more narrowly textual modes of investigation by making use of in-depth interviews and ethnographic forms of investigation such as participation-observation, and by investigating the structural features of the contexts that limit, but do not determine, social and political possibilities. ${ }^{18}$

In addition, Howarth argues that Post-Marxist discourse analysis also accounts for issues such as resistance and conflict without having to rely on distinctions of class. Such problems are instead treated by the Laclauian discourse theory as a result of discursive shifts that prevent social groups from realizing their identity. When identities are attacked, social

\footnotetext{
${ }^{18}$ Howarth nonetheless regards all of these media as "text" (ibid., 10); a view that I argue obscures the various dynamics of visual and acoustic communication. An alternative approach to such media is provided in Chapter 2.4 
agents are compelled to act. In this sense, the work of Post-Marxist authors suffers from an overemphasis on social struggle similar to the one inherent to CDA. Such a focus presupposes that social relations are primarily characterized by constant antagonistic struggles for domination, and motivated by conscious efforts of social actors to assert their discursive position. As Dreyfus and Rabinow show (1983, 169 \& 186-187), such an understanding of social interaction runs contrary to Foucault's vision of power, knowledge, and discourse. For this reason the approach introduced in this thesis does not attempt to trace forms of contestation in popular Chinese media discourses, but instead focuses on social practices that make certain interpretations of concepts such as security or nationality seem "normal". The theoretical axioms behind this reasoning are that:

1) although all social interaction is governed by power, the actual mesh of power relations is not consciously motivated by certain classes or groups, but instead possesses its own logic, based on wider social practices;

2) while subjects construct discourse, discourse in turn constructs both its objects and its subjects.

In order to analyse the normalizing effects that mass communication has on political discourse and at the same time retain the original radically constructivist concept of discourse proposed by Michel Foucault, my approach rejects various concepts used in other forms of discourse analysis, such as the Marxist notion of ideology, the cultural studies focus on representation and mis-representation of an objective truth, the view that all social practices should be equated with "text", or the Post-Marxist premise that the defining elements of discourse are struggle and antagonism. Instead, the analytical approach used for this project combines the general theoretical framework of Foucauldian discourse analysis as 
summarized by Dreyfus and Rabinow (1983) with the methodological toolbox of the German critical discourse analyst Siegfried Jäger (2004). ${ }^{19}$

\subsection{Discourse and the Building Blocks of Reallty}

If we accept the premise that discourse is, as Jäger writes, "the flow of knowledge through time" (Jagger, 2004, 129; author's translation), established and reinforced through communication practices, then this leads to two questions: first, what does this "knowledge flow" encompasses (i.e. what is it constructed from)? And second, what factors define its flow (i.e. how is it constructed)? The fundamental building blocks of discourse are, to use Jäger's terminology, the discourse strand, the discourse fragment, and the discourse layer.

The term discourse strand refers to specific themes or topics (ibid., 160-163). Allow me to give an example: the topic of globalization is a discourse strand; it brings together all knowledge concerning the concept of globalization, thereby creating the 'truth' of what globalization is. The various discourse strands do not exist separate from each other, but are interwoven: for example, the globalization strand intersects with strands such as migration, crime, law enforcement, labour, gender, ethnicity, religion, and many others. In this sense, what we hold to be true about globalization may also influence what we hold to be true about the nature of crime, and in turn may be influenced by those conceptions. All discourse strands together form the discourse in its entirety, i.e. everything that is known and believed to be true throughout society.

Discourse strands consist of yet smaller building blocks, the discourse fragments (ibid., 166168): The term 'fragment' refers to statements, or claims of truth, concerning a discursive theme, and they can be made both consciously and unconsciously. To again give an example,

\footnotetext{
${ }^{19}$ This is not to say that the present research approach stands in stark opposition to the approaches developed by CDA researchers or discourse analysts such as Fairclough, van Dijk, or Howarth. As Howarth himself states, discourse analysis needs to be an open approach: "...instead of applying theory mechanically to empirical objects. or testing theories against empirical reality, discourse theorists argue for the articulation and modification of concepts and logics in eech particular research context" (Howarth, 2000, 139).
} 
within the discourse strand of globalization, the statement globalization brings the people of the world closer together is just as much a discourse fragment as the statement globalization has brought about the Clash of Civilizations, or because of globalization, immigrants are taking away our jobs. From a discourse analytical perspective, any such statement made concerning globalization is part of that particular discourse strand, consequently contributing to the construction of the 'truth' about globalization. In addition, any notion that has not been voiced concerning globalization is not part of that discourse strand. Such hypothetical statements would exist outside of discourse, denying us their use in our communication practices, which in discourse terms means that they do not exist at all.

As mentioned above (2.3.i), the discourse fragments that are most widely accepted throughout society are said to be part of the hegemonic discourse (i.e. the dominant world view throughout society, held in place by power dynamics). Statements that diverge from this mainstream view of the world are said to be part of a counter-hegemonic discourse (a worldview that challenges the dominant interpretation of truth). For example, we could say that the neoliberal statement open markets benefit society is part of the hegemonic globalization discourse, whereas the statement globalization marginalises certain social groups is associated with the counter-hegemonic discourse on globalization (of course such relations may change, and some might argue that the later of the above statements is actually becoming more of a hegemonic than a counter-hegemonic one). The diverse array of discourse fragments that we each use in our communication practices define our individual perception of what is true, i.e. our discursive position (ibid., 163).

Finally, discourse has various layers, meaning that communication practices can take place in different settings (ibid., 163-165). Allow me to provide another example: discussions concerning globalization produce certain statements in an academic setting, but other statements in the media, and yet other statements in the privacy of our homes. In this sense, 
the discourse strand of globalization has an academic layer, a media layer, a domestic layer, and many more. Just like the various discourse strands interact with each other, so do the discourse layers influence one another: for instance, the discourse fragment that globalization brings about the Clash of Civilization can be said to have originated in the academic discourse layer (with the publication of Samuel Huntington's now famous book "The Clash of Civilizations"; Huntington, 1996), but to now have made its way into all other layers of the discourse strand on globalization (for example by being referenced in the media, or possibly in a private dinner conversation, whether consciously or unconsciously).

\section{3.v 'Synchronic' vs. 'Dlachronic' Discourse Analysis}

Returning to Jäger's imagery of viewing discourse as the flow of knowledge through time, we can generally differentiate between two different types of discourse analysis. The first is what Jäger refers to as synchronic sectional examination of a discourse strand (Jäger, 2004, 171). This is the analysis of the statements that are made concerning a specific topic at one point in time. Jăger himself offers a comprehensive example of how bio-political issues were discussed in Germany's most popular tabloid newspaper, the BILD, in the year 1994 (ibid., 321-363).

The second method of analysis is the so-called diachronic discourse analysis in which individual sections of the discourse strand are compared over time (ibid., 171). This was Foucault's approach: he focused not only on what was said at different points in history, but also on who could make such historical statements, from what institutional position they were made, what social context gave rise to their formulation, and consequently how these conditions changed over time. In his early work, Foucault gives the example of medical diagnostic statements in western society (Foucault, 2005, 55-60): He describes how "medical statements cannot come from anybody" and how only a person who can claim to have the power to overcome suffering and death (i.e. a doctor) may offer advice or treatment. 
Foucault continues to show how, on the one hand, the institutional setting of the hospital (with its laboratories and libraries) and on the other hand the role of the medical practitioner (along with his training and the established methods of diagnostics and treatment he makes use of) define the array of statements he can make; Foucault then goes on to describe how such statements along with their socio-historical background changed throughout the $18^{\text {th }}$ and $19^{\text {th }}$ century (ibid.). A similarly diachronic approach characterizes Foucault's later work, in which he traces the changes in disciplinary techniques (Foucault, 1995) and of conceptions of sexuality since the $1^{\text {th }}$ century (Foucault, 1990).

A historically diachronic analysis of discourse is a challenging project that should only be conducted by researchers with substantial resources and an extended time frame; as Jäger points out, it is not a feasible undertaking for a single research thesis (Jäger, 2004, 188). I will therefore focus on a synchronic analysis of discourse, i.e. on what is being said in specific popular television programmes in China at a specific time. For this purpose I have chosen television series that were broadcasted during the random time interval of the years 2003 to 2005 (see also Section 2.6.i).

\section{3.vI A Methodology of Critical Discourse Fino-Analysis}

In order to conduct a critical discourse analysis, or what Jäger refers to as "discursive fineanalysis" (ibid., 175; meaning: a detailed analyses of the discourse fragments represented in individual media products, such as newspaper articles, television dramas, etc.), a comprehensive methodological framework is required. But before turning to the tools that discourse analysts apply, a look at the analytical questions that they most commonly address is in order. Generally speaking, the central research questions of critical discourse analysis are (ibid., 185): 
1. What is the message of the product (the motives and intentions behind the product)?

2. Who is the author or producer of the statements made in the product (i.e. who is speaking)?

3. What are the linguistic and rhetorical methods used by this author?

4. Who is the target audience?

5. What change of world views is the product supposed to bring about? Are the discourse fragments aimed at reaffirming old truths (i.e. are they part of the hegemonic discourse) or are they meant to introduce new truths (i.e. are they part of the counter-hegemonic discourse)?

6. What is the social context in which the product has been created?

7. How does the text refer to or make use of political, economical, historical or cultural events (so called 'discursive events')?

To answer these questions Jäger suggests conducting a fine-analysis of the particular

products. He offers the following open five-step-framework of analytical methods (ibid., 175):

1. Analysis of the institutional context: This includes defining the medium and the category of the product, the author(s) and any possible events which the product refers to, but also what could be called the production process. Jäger gives the example of news articles, which should be defined according to the newspaper that they are published in (along with information concerning the editorial staff, general political position of the paper, its affiliation with other organizations, production expenditures and general finances, target audiences, etc.), the genre of the article (commentary, news, report, etc.), but also according to its technical quality (paper, type set, readability, number of pages, etc.; ibid.).

2. Analysis of the 'text surface': This stage of analysis is concerned with graphical configurations of the product (in the case of a newspaper article this may include the analysis of photos, graphics, headlines, etc.), with its structure (paragraphs, sections, subsections, etc.), and with the particular topics discussed. At this stage it may be useful to prepare the product for further analysis by numbering the lines of the text, summarizing the content, marking structural segments according to their function and meaning, and so on (ibid.).

3. Analysis of linguistic and rhetoric tools: This is the actual core of a fineanalysis. It includes the examination of arguments, of the products logical composition, of implications, conclusions, allusions, metaphors, idioms, proverbs, etc. It also focuses on the general style of the product, on protagonists and antagonists, on vocabulary (nouns, adjectives, verbs, adverbs, pronouns, etc.), on collective symbols as well as on references and intertextuality. The preparation of this analytical phase is probably the most elaborate work step in a discourse analysis. Jager shows what 
detail is required in dissecting a qualitative interview (ibid., 275-328): Every statement has to be categorised according to content and context, communication strategies need to be identified, and idioms classified. In his example (an interview with a German couple conducted in 1992, analysed for racist statements), Jäger is able to identify over one hundred different racially charged idioms. He analyses twenty-six separate narrative strategies (such as a generalization about gangs of Turkish immigrants or about Jewish money lenders) and goes on to show how the interviewees use pronouns (" $\mathrm{I}$ ", "we", "they") to differentiate between themselves and others, thereby avoiding direct references to specific ethnic groups ("we Germans", "those Turks", etc.). Jäger's analysis also focuses on metaphors, collective symbols and syntactic strategies used by the interviewees (such as the strategy of giving information, of appealing to the interviewer, of declaring an opinion, etc.). It may be useful to apply some quantitative methods during this section of the analysis, for example computer searches for keywords. This may "make it possible to draw conclusions concerning the emphasis on topics or the shifting of discourses", but should, according to Jäger, never replace the qualitative dimension of the analysis (ibid., 197).

4. Analysis of content and discursive positions: As a fourth step, the product needs to be analysed for discursive positions, such as the general worldview expressed by the author(s) as well as the text-immanent understanding of such concepts as politics, society, technology, history, future, gender, race, etc. (ibid., 175).

5. Interpretation: After the above four analytical steps have been completed, an actual coherent description and interpretation of the product can be composed. The different elements and results of the fine-analysis need to be related to each other, the discourse analytical questions cited at the beginning of this section have to be addressed, and a final conclusion needs to be drawn. As a sample conclusion I would like to cite from the interpretation of the bio-political discourse in the German BILD newspaper as conducted by Jäger and a group of other German discourse researchers: "The bio-political discourse contributes to keeping the reader small and faint. One is supposed to be scared, not think on one's own. In this way, it is not only possible to describe a beautiful passing-away, but also to show gruesome deaths due to accidents or cancer in all their harshness. On the one hand, BILD fuels fears; on the other hand, it puts the cruelty of death into perspective. That way, BILD can praise peaceful death and at the same time frighten readers with the horrors of mistaken organ transplants from patients in suspended animation" (ibid., 361; author's translation).

These methods need not all be applied to a single discourse analytical project. Jäger calls this set of methods a "toolbox" and points out that researchers should choose freely the instruments they find most useful depending on their particular research question (ibid., 159). 


\section{3.vil Analysing Political Discourse Strands}

Since this research project is concerned with the analysis of political discourse, it is first necessary to clarify what the term 'political discourse' refers to, and then define which political discourses shall be analysed here. By political discourse I mean any discourse strand that deals with any of the classical issues of politics, i.e. the "art and science of government" (McLean et al., 2003, 423), or more precisely "regularities concerning the inner order and the actual functioning of systems of governance" in respect to their form (polity), procedures (politics), and results (policies; Schmidt, 1995, 907; author's translation).

Traditionally, the main organization relevant in politics is the state (McLean et al., 2003, 512; Zürn, 1998, 37). The term state originally signifies a condition of political order under which a group of persons are bound to a territorial region and controlled by sovereign rule (Evans et al., 1998, 512; Schmidt, 1995, 896). According to the German political scientist Michael Zürn, states have three characteristics (Zürn, 1998, 37-38): a state possesses a legitimate monopoly of force that gives it the autonomy to acquire resources across a territory; it follows goals in order to achieve a certain degree of public welfare; and it is recognised by other states (for similar conceptions of the state in Anglo-Saxon political science literature, see Evans et al., 1998, 512 and McLean et al., 2003, 512).

In this sense, the state is traditionally regarded as the main institution that regulates society. The term governance refers to the measures that the state or other institutions adopts in order to do so (Evans et al., 1998, 209; Zürn, 1998, 35-40).20 More recently, the question has been posed whether the institution of the state is actually the best entity to fulfil this governance function (Zün, 1998, 165-176). Authors such as Peter Taylor (1996) criticize that the concept of territoriality that is associated with statehood is outdated. Also, as Susan

\footnotetext{
${ }^{20}$ The modern concept of governance has its origins in German public administration literature and system theory; the British political scientist Bob Jessop (1998) gives an overview of these roots and of the various German researchers, such as Grabher, Luhmann, Messner, Scharpf, or Wilke, who have influenced the Anglo-Saxon understanding of the word.
} 
Strange's analysis of the world economy shows, the state as a political entity is increasingly being replaced by new power dynamics (1996, 91-179). Similarly, Andrew Gamble $(2000,114)$ writes that due to the rising complexity of political issues and the increased proneness of states to yield policy failures, the institution of the state today primarily plays "an enabling rather than a planning" role in social regulatory activities. Gamble argues that the state's task is now mostly to support "markets and networks or corporate hierarchies in governing the economy, rather than to seek to impose its own pattern or its own agenda" (ibid.).

Such considerations have lead to a broader understanding of the term governance, which "embraces governmental institutions, but (..) also covers more informal regulatory mechanisms which exist in the absence of central authority" (Evans et al., 1998, 209). Andrew Gamble shows in his analysis of governance in the European Union $(2006,42-44)$ that the concept can be understood either in a narrow, territorial sense (i.e. mostly referring to the regulatory activities of the nation state and its institutions) or in the broader sense of multilevel network governance, meaning: "steering mechanisms that involve networks, markets and other decentralized forms of co-ordination across legal jurisdictions and administrative boundaries" (ibid., 43). Wider interpretations of governance often turn towards civil society and its relation to the state, arguing that civil agents can regulate aspects of society apart from, against, in support of, in dialogue with, in partnership with, or beyond the state (Chambers et al., 2008, 364). Gerry Stokes, former director of the British Economic and Social Research Council's Local Governance Research Programme, writes that "governance is about a change in the long-standing balance between the state and civil society" (1998, 21). He describes how such a change can blur the distinction between the public and the private sectors in society (ibid., 17). Consequently, Stokes argues, the increased flexibility of nonstate governance is accompanied by legitimacy and accountability deficits, possible blame avoidance or scapegoating, potential unintended outcomes, and a continued risk of 
governance failure (ibid., 19). Similar concerns are raised by Bob Jessop from Lancaster University (1998, 38-42), who further points out that many governance shortcomings are not actually consequences of state failure, but instead have their roots in the logic of modern capitalism $(1997,573-577)$. To counter such tendencies, Jessop suggests a stronger role for governmental institutions, which he believes should create a non-hierarchical framework for regulatory activities, or in his words: "meta-governance" (ibid., 575). The state, so the argument goes, should take responsibility for overarching political regulation and should coordinate the networks and agents involved in acts of governance (Jessop, 1998, 42-43).

Whichever actual form mechanisms for steering society take, they generally function through the use of power. The term stems from the Latin noun potentia and signifies an ability, faculty, or potential (Brunner, 2004, 836). This notion of capability is reflected in the definition of power by sociologist Max Weber, who refers to power as "any chance of enforcing one's own will in a social relationship, even against reluctance, regardless of what that chance may be based on" (Weber, 1980, 28; author's translation; this definition is essentially also used by Strange, 1996, 17 and 25). In short, power describes the ability to prevail, and in the present context refers to the ability of public and private agents to achieve the goals of governance.

Governance generally follows four such goals (Zün, 1998, 41-42). The first of these goals is to provide security for the population of a specific territory (ibid., 41; Cerny, 1996, 123; Stoker, 1998, 17; Strange, 1996, 84); in Europe this goal dates back to the time of the feudal system, and its achievement is often regarded as the minimal activity required of the state (McLean et al., 2003, 515; Zürn, 1998, 40-44). The need for security traditionally justifies the use of force both within a territory in order to secure stability and protect the population against crime (inner security), as well as outside of that territory to protect it against foreign aggression (outer security). Zurn shows that it was this requirement for security which made the rise of European territorial states in the $12^{\text {th }}$ and $13^{\text {th }}$ century possible and, paradoxically, 
caused the continuous increase in the need for outer security over the centuries due to states' growing ambitions for territorial expansion (ibid., for another critique of states' inherent inclination to use force in order to secure their sovereign territory see Giddens, 1994, 231).

A second objective of governance is the goal of welfare (Cerny 1996, 128; Marshall, 1975, 11 12; Zürn, 1998, 48-54): In the modern state, welfare not only implies economic success, i.e. high levels of national productivity, maximum employment, optimal resource-allocation and production-distribution as well as economic growth; it also includes provisions in such areas as education, health and environmental protection.

The third goal of governance is that of creating and maintaining national identity (McLean et al., 2003, 515; Zürn, 1998, 44-46): This goal is relevant in order to assure that the citizens of a state associate themselves with the entity that governs their society, and is achieved by recourse to a collective cultural, historical or ethnical background.

A fourth goal of governance is legitimacy (McLean et al., 2003, 515; Stoker, 1998, 20-21; Zürn, 1998, 46-48). Some authors, such as Michael Zürn, understand legitimacy as essentially the western liberal-democratic rights of citizenship and participation that have come to play a central role in democratic welfare states (Zürn, 1998, 46-48). Such a notion of legitimacy is not unproblematic. To narrow down legitimacy to a concern of Western democratic welfare states, and to give it an entirely normative meaning (i.e. asking whether or not governance is good governance), neglects the fact that political institutions in general need to justify their political activities (McLean et al., 2003, 305-306). This idea is reflected in Max Weber's use of the term legitimacy, which I shall be using here (Weber, 1980, 122-176): For Weber, the central question of legitimacy was functional in nature, not normative; he asked how a sovereign justified the claim for authority, not whether or not that claim was valid. For example, a system of governance can be justified through certain traditions, through the charismatic qualities of a leader, or by adopting a certain legal system (ibid.). What is 
important to realise is that political institutions, such as states, all aim at justifying their existence and their activities. This is acknowledged by Philip Cerny when he writes that the essence of the state "lies in the fact that sovereign and autonomous political institutions are capable of deriving legitimacy from a distinct citizenry located in a defined territory" (1996, 123; for a similar understanding of the term legitimacy see also McLean et al., 2003, 305-306; Stokes, 1998, 20; and Strange, 1996, 71-72).

The present analysis of political discourses in Chinese television dramas takes as its starting point the discourse strands on governance and its four goals (security, welfare, identity and legitimacy). For reasons of feasibility, the complexity was reduced by selecting only two of these strands for detailed fine-analysis: The discourse on governance, and that on the first goal of governance, i.e. security. However, even though the analysis presented in Chapters $4.2,4.3$, and 4.4 solely focuses on these two political topics, the coding procedure used to identify the discourse strands presented in the respective drama series nonetheless used categories devised from all of the discourse strands discussed above (for an explanation of how the coding categories were devised see section 2.6.ii). This was done in order to assure that the possible interaction of different discourse strands that Jäger describes $(2004,161)$ is not missed in the analysis (for instance in cases where the discourse strand on public security interacts with the discourse strand on national identity).

\subsection{The Theory of Visual Communication}

The methodological practices described so far offer many suggestions on how to analyse discourse, but broad approaches such as the one offered by Jäger are mostly concerned with language and its social setting. The analysis of visual components is rarely featured very prominently in the works of discourse researchers (for example, Jäger's analysis of the BILD newspaper, a tabloid which makes substantial use of photos and montages, hardly touches on the importance of visual images at all; 2004, 329-263). This is quite surprising, considering 
that a number of very useful tools have been developed in order to study the meaning of images. The German political scientist Marion Müller, for example, has summarised the most common methods in her book "Basics of Visual Communication". ${ }^{21}$ In her own research, Maller explores, among other things, how the BILD newspaper uses photos of deceased in different contexts $(2003,84-85)$ : depending on whether the person shown is depicted as a victim or as a perpetrator, the paper shows either a frontal shot (in order to make the reader identify with the deceased) or a profile shot (to create a distance between the viewer and the depicted person).

Although Muller does not use the term 'discourse' in her work, her methodological approach nonetheless bears a certain similarity to that of discourse specialists such as Jäger, and some of her analyses (for instance her examination of how former German Foreign Minister Joschka Fischer's alleged involvement in violent demonstrations during the 1970s was visually reworked in 2001 by the conservative German press and the opposition party to associate Fischer's party with violence and chaos) could be called discourse analyses (ibid., 88-91). The proximity to discourse analytical approaches is also apparent in other visual analyses, for instance in Gombrich's research on how blurred images are used to suggest that their content is particularly 'true' (1980). I therefore argue that such concepts of images as a type of communication, as well as the notion that images signify meaning which can be tested and analysed, can be quite readily integrated into the framework of discursive fine analyses which I have described above.

\subsection{Understanding Images as Purveyors of Meaning}

Marion Muller's concept of the image is essentially this: that an image constitutes a visual representation of a material thing and that this material image is intricately linked to a mental image of that material thing $(2003,20-25)$. This idea that images represent certain

\footnotetext{
${ }^{21}$ For more examples on the political analysis of images see Hoffman (1999), Múller (1999), and Warnke (1994).
} 
things or ideas bears a certain resemblance to semiotic approaches. For practical reasons, Müller herself abandons the rather technical and often abstract notions that many semioticians hold concerning visual communication (ibid., 162), but also concludes that semiotic approaches can be fruitful for questions "that deal with mixed forms of communication in which audio, verbal and visual processes are intertwined, such as for example in the case of film analyses" (ibid., 163; author's translation). Since the present analysis focuses precisely on such a multi-dimensional form of communication, it is worth considering some of the semiotic ideas that have been developed concerning the question of how meaning is purveyed in such communication processes.

The essential concept in semiotics is that messages are communicated through a series of signs, or as Ellen Seiter writes (1992, 31): "Semiotics is the study of everything that can be used for communication: words, images, traffic signs, flowers, music, medical symptoms, and much more. Semiotics studies the way such 'signs' communicate and the rules that govern their use."

One definition of the sign was provided by the French linguist Ferdinand de Saussure (for an overview of Saussure's life and work see Preucel, 2006, 21-43). Saussure, being mainly interested in the functioning of language, defined the sign as a "two-sided psychological entity" that links a mental concept of a thing, i.e. the 'signified', to a sound pattern, i.e. the 'signifier' (ibid., 28). In other words, when human beings verbally formulate an expression to communicate an idea, we are confronted with a sign. This concept of the sign is not unproblematic (Eco, 1979, 15; Hoopes, 1991, 11): since Saussure was analysing only language, he assumed that the relation between signified and signifier had to always be entirely arbitrary. This can be observed when various languages employ entirely different terms for the same thing, while none of these words in any way resembles the actual thing (for instance when water pours from the sky, the English signify such a phenomenon by using the word 
'rain', while the Germans use the term 'Regen', and the French the word 'pluie'; Seiter, 1992, 14). Based on this observation, Saussure excluded from his analysis all non-verbal impulses that could also be used to signify meaning and communicate a message (such as, for example, an image of rain), on the other hand he excluded any signals that were not intentionally and artificially articulated by human beings for communication purposes, but could also become carriers of meaning (for instance, Saussure would not have given any importance to the idea that the sound of rain falling on the roof of a house can also signify 'rain' to those inside).

A more comprehensive definition of signs was provided by the American logician and pragmatist Charles Peirce, who formulates that a sign is (1991b, 239):

Anything which determines something else (its interpretant) to refer to an object to which itself refers (its object) in the same way, the interpretant becoming in turn a sign, and so on ad infinitum.

By using this definition, Peirce establishes a triangle in which the sign (or 'referent') is linked to the thing it stands for (the 'object') as well as to its interpretation. Note how interpretant does not mean interpreter, but rather "that which guarantees the validity of the sign, even in the absence of the interpreter" (Eco, 1979, 68); this makes it possible to view various different denotations or connotations of a sign as interpretants, which then in turn can become signs in further chains of communication (ibid.).

Peirce definition is fundamentally different from Saussure's, as is explained by the Peircescholar James Hoopes (1991, 11-12):

While words may be arbitrary in some respect, verbal language is far too narrow a field from which to construct a general theory of signs, as becomes evident from the arbitrariness posited of the social world by those who suppose that it is constituted in language. Peirce's theory, based on an analysis of thought rather than language (in the narrow, verbal sense), posits within the signifying process not only an object and its sign but also a third element, the interpretant, or thought, to which the sign gives rise. 
Resorting to Peirce's definition now enables us to regard signs as much more than verbal expressions. In addition, it assures that the relation between the sign and the object it stands for is not understood as random, or as Peirce puts it (1991a, 141): "a sign must have some real connection with the thing it signifies." He himself gives the following example (ibid):

A weathercock is a sign of the direction of the wind. It would not be so unless the wind made it turn round. There is to be such a physical connection between every sign and its object.

Peirce then goes on to classify three different types of signs, depending on their particular relationship with the object and the interpretant. Peirce defines these types of signs as follows (1991b, 239-240):

A sign is either an icon, an index, or a symbol. An icon is a sign which would possess the character which renders it significant, even though its object had no existence; such as a lead pencil streak as representing a geometrical line. An index is a sign which would, at once, lose the character which makes it a sign if its object were removed, but would not lose that character if there were no interpretant. Such, for instance, is a piece of mould with a bullet-hole in it as a sign of a shot; for without the shot there would have been no hole; but there is a hole there, whether anybody has the sense to attribute it to a shot or not. A symbol is a sign which would lose the character which renders it a sign if there were no interpretant. Such is any utterance of speech which signifies what it does only by virtue of its being understood to have that signification.

In other words, the $i c 0$ bears a resemblance to its object, such as a drawing of a horse signifying a horse. An index is the consequence or symptom of its object (and therefore requires the presence of its object to become a sign), such as smoke signifying fire, or lightning signifying a thunderstorm. Finally, a symbol is a sign that depends on its interpretant in order to become a sign, such as a stop sign signifying 'stop' only because social conventions has taught people to treat it as such a sign.

This division of signs into three types may lead some to believe that symbols are the arbitrary signs that Saussure was referring to (and possibly interpret them as 'subjective'). while understanding indices and icons as objectively real signs that are based on objects in the material world (and therefore possibly conclude that they are 'truer' than symbols). 
However, such a simplification would be misleading, as the Italian novelist and semiotician Umberto Eco shows. Eco argues that all signs are part of a social system of rules, or what he calls codes. A code defines unambiguously what the content of the sign is. A sign is related to content by means of code, and this relation between sign and content is called a signfunction $(E c 0,1979,191)$ :

...a sign-function is the correlation between an expression and a content based on a conventionally established code (a system of correlational rules) and (..) codes provide the rules that generate sign functions.

This means that just because a symbol, for instance a word that is not referring to a specific physical object (such as freedom or peace), is based on social conventions does not mean that it is less real or less true for those who use it (ibid., 62):

In order to understand the history of Christian theology, it is not necessary to know whether a specific actual phenomenon corresponds to the word /transubstantiation/ (even though for many people this belief was vitally important). But it is necessary to know which cultural unit (what intensionally analyzable set of cultural properties) corresponded to the content of that word.

The semiotic object of a semantics is the content, not the referent, and the content has to be defined as a cultural unit (or as a cluster or a system of interconnected cultural units).

Conversely, indices may be referents to physical objects in the material world, but they

nonetheless carry content in a manner that is similarly based on social conventions:

The first doctor who discovered a sort of constant relationship between an array of red spots on the patient's face and a given disease (measles) made an inference: but insofar as this relationship has been made conventional and has been registered as such in medical treatises a semiotic convention has been established. There is a sign every time a human group decides to use and to recognize something as the vehicle of something else.

Smoke is only a sign of fire to the extent that fire is not actually perceived along with the smoke: but smoke can be a sign-vehicle standing for a nonvisible fire, providing that a social rule has necessarily and usually associated smoke with fire.

As another example Eco explains that the Evening Star in the night sky is only relevant because of the code that governs the semiotic event of a person recognizing it as such (ibid., 
66): the light of the star may be an index denoting "a certain large physical 'object' of a

spherical form, which travels through space some scores of millions of miles from the earth", but to the person seeing that light, it denotes "'a certain' corresponding cultural unit to which the speaker refers, and which he has accepted in the way described by the culture in which he lives, without having ever experienced the real referent" (ibid.); in this sense, the light in the sky may be the Evening Star to one person, and the Morning Star to someone using a different set of cultural units (i.e. a different code):

We may commonly speak of a thing called/Alpha Centauri/, but we have never experienced it. An astronomer has occasionally experienced it with some strange apparatus. But we do not know this astronomer. We only know a cultural unit communicated to us by means of words, drawings or other means. (ibid.)

In this sense, even an icon such as an image, i.e. the visual representation of the 'real thing', is based on a code, and therefore on social conventions (ibid., 199):

...what matters is not the relationship between the image and its object but rather that between an image and its content. The content, in this case, is the result of a convention, as is the proportional correlation. The elements of motivation exist, but they can only work when they have been conventionally accepted and coded. 2

Even though an icon is similar to its object, this does not change the fact that "similitude is produced and must be learned" (ibid., 200).

In order to capture the notion that all signs are socially constructed, Umberto Eco formulates his own definition of the sign, and this definition shall be the basis for the analysis provided in this thesis (ibid., 16):

I propose to define as a sign everything that, on the grounds of a previously established social convention, can be taken as something standing for something else.

\footnotetext{
${ }^{22}$ What Umberto Eco means by "motivation" is that the use of signs and codes requires human action. He explains: "Everybody who wants to know something wants to know it in order to do something. If he claims that he wants to know it only in order 'to know' and not in order 'to do' it means that he wants to know it in order to do nothing, which is in fact a surreptitious way of doing something, lie. leaving the world just as it is (or as his approach assumes that it ought to be) $)^{n}($ Eco, 1979, 29).
} 
In addition to the idea that signs have a social dimension, it is important to realise that they themselves also possess constructivist power. Eco gives examples of different codes and how they structure the reality of those who use them: for example, the fact that the Latin language has only one word signifying both "rat" and "mouse", while the English language has two words, means that from a semiotic perspective "there exists in English a semantic field governing rodents, which is more analytic than its equivalent in Latin, and that therefore for the speaker of English there exist two cultural units where for the speaker of Latin there exists only one" (ibid., 79). Similarly, we can divide the spectrum of light into various sections of different wavelengths, thereby differentiating between various colours; however, as Eco shows (ibid., 77-78), Hindu culture combines under one term what in European culture would be perceived as red and orange, while Russian culture subdivides the European concept of blue into two distinct colours (a light blue and a dark blue). In that sense, people with different cultural backgrounds see the same colour spectrum, but experience a different reality of colours (i.e. of cultural units). It is this notion that signs have a social dimension that leads Eco to the conclusion: "Semiotics is mainly concerned with signs as social force" (ibid., 65). It is also this notion that makes it possible to integrate semiotic theory into discourse theory. With discourse being communication practices that construct reality, and signs being cultural signification units that make up communication practices, it is only logical to view signs as the building blocks of discourse.

\subsection{How Signs Work within Visual Communication}

In the present analysis, I shall view discourse as consisting of discourse fragments, which in turn are composed of a series of signs. Considering the above explanation on signs, and particularly Peirce's categorization of signs into icons, indices and symbols, one might now assume that the study of visual communication practices such as moving images is primarily concerned with the analysis of icons, i.e. of signs that resemble their object. However, this 
would not fully capture the various dimensions at which a medium such as television functions (Seiter, 1992, 35):

The categories symbolic, iconic, and indexical are not mutually exclusive. Television images are both iconic and indexical, and programmes often use words (symbolic signs) on the screen and the soundtrack.

The question then is: how do signs compose a complex message such as the one we find in a television drama series? The answer is provided on the one hand by Umberto Eco's concepts of denotation and connotation, on the other hand by Roland Barthes considerations of how these concepts apply to multi-dimensional processes of mass communication.

First, let me explain what the difference between denotation and connotation is according to Umberto Eco's theory of semiotics. Eco gives the example of a particular communication process $(1979,32-40 \& 54-57)$ in which a machine measures the level of water at a dam (the 'source') in four intervals and then 'codes' the respective intervals with a combination of two letters $(A B, B C, C D$ and $A D)$. The machine (or 'transmitter') then transmits the two-letter expression (the 'signal') to a station in the valley (the 'destination'), where an engineer interprets the signal (thereby making it a 'sign'). What happens in this example is that the decoding of the sign happens at two levels: first, the sign denotes something; for example, the letter combination $\mathrm{AB}$ directly stands for 'danger level'. This kind of semiotic relation is called a denotation. Second, the denotation 'danger level' in turn stands for something else: in this case it signifies an appropriate response for the engineer; i.e., the letter combination $\mathrm{AB}$ indirectly stands for 'evacuation of water'. This kind of semiotic relation is called a connotation. Eco writes (ibid., 55):

There is a connotative semiotics when there is a semiotics whose expression plane is another semiotics. In the above example the content of the former signification (along with the units that conveyed it) becomes the expression of a further content. Thus the expression $\mathrm{AB}$ denotes "danger level» and connotes «evacuation". 
In this sense, connotation is the signification of a signification, or a sign-function within a sign-function. Eco explains this as follows (ibid., 56):

...the difference between denotation and connotation is only due to a coding convention (...): once the connotation has been established, the connotation is the stable functive of a sign-function of which the underlying functive is another sign function. A connotative code, insofar as it relies on a more basic one, can be called a subcode.

The concept of connotation provides one challenge, however, and that is that signs can be ambivalent. As Eco shows, one denotation can connote more than one thing, for instance 'danger level' can signify both 'evacuation' and 'flood' for the engineer (ibid., 56). In this particular case, the two connotations are not mutually exclusive, but one can easily think of examples in which connotations clash. Allow me to give a hypothetical example: Imagine the image of a car. The image denotes a particular automobile, say a powerful off-road vehicle. The image of that car may now connote various things: for one person the idea of the car may stand for freedom, while for another person it may signify environmental degradation. The question then is: Is one connotation more relevant than the other?

The answer is again provided by the code. One should not mistake the ambivalence of connotations with arbitrariness. Connotations are culturally constructed. Allow me to provide another hypothetical example: Imagine a Chinese advertisement for cosmetics that features a woman with very pale skin; in this particular context, the skin tone connotes beauty (and, at one level removed, further connotes prosperity). This connotation is based on a code of cultural units, which associates tanned skin with the type of physical outdoor labour that peasants and workers perform. Hence this connotation would hold anytime pale skin was featured in the promotion of cosmetic products in China. However, if we were watching a similar commercial in a Western country, the pale skin tone would connote 'unhealthyness', since in that culture the code would link the sign of tanned skin to beauty and prosperity (based on the association of tanned skin with leisure time for outdoor 
activity). The different connotations are not arbitrary, but what is required to understand them is knowledge of the code that led to their formation (ibid., 72):

... cultural units are the semiotic postulate required in order to justify the very fact that society does equate codes with codes, sign-vehicles with meanings, expressions with contents. Unseen but used by the layman, they are not used but seen by the semiotics, which is simply the science of this culturally performed (if unexpressed) competence.

This concept of connotation as the socially coded content of a denoted message may lead some to assume that the difference between denotation and connotation is in fact the same as between objective and subjective signification. However, Eco shows that denotations are just as much based on social and cultural conventions as connotations. He gives the example of a black pencil line sketching the outlines of a horse (ibid., 193-194): The black pencil line will only be recognised as the image of a horse if it adheres to certain conventions. Similarly, Eco demonstrates how flawed drawings of rhinos from 18th century Europe had such a cultural impact on society at the time that for over a century they would define the 'reality' of what rhino-skin looked like, even though other more accurate images were soon available (ibid., 205). One may argue that images only follow cultural conventions in the case of drawings or paintings, but that a photo actually represents 'the real thing'. This is also not the case. The mere fact that we flatten a three-dimensional experience to two dimensions, place it in the rectangular from of the photo (Snyder, 1980, 225), but still recognise its denotation, is based on a cultural convention, or as Ellen Seiter put is $(1992,37)$ :

Conventional expectations of scale, perspective, camera angle, color, lighting, lens focal length, and subject-to-camera distance (that is, non-representational aspects of the image) are acquired through exposure to television; if a camera operator violates too many of these conventions, we may not be able to 'recognize' the image at all.

This is amply proven by the fact that children have to first learn how to decode photos and television images (Barthes, 1977, 36); man is not born with this ability, and the denotative value of photos and motion pictures is not as intuitive as one might believe. Allow me to 
give another example, that of the various conventionalised camera angles that are used in motion pictures. The only angle that could in some sense be called "natural" is the so-called point-of-view shot (POV shot), which lets the viewer see through the eyes of one of the characters, suggesting that he or she is actually taking part in the action (such a shot of course only simulates naturalness; there is very little that is natural about looking through the eyes of another person). But let us consider another camera angle, one of the most-often used angles in film-making: the so-called over-the-shoulder shot (OTS shot). In such a shot, the viewer is placed next to one of the characters in a dialogue, looking over that character's shoulder at the person he or she is talking to. The angle is then reversed to place the viewer next to the other person, looking over their shoulder back at the first character. This technique has become so conventionalised that most viewers will hardly notice it when watching a dialogue scene on television. However, there is nothing about this angle that is natural or intuitive: the space that the camera fills in order to provide this shot is actually not held by any character in the motion picture so that once the shot is reversed one would normally have to wonder where the perspective was actually coming from. In addition, the OTS shot is a perspective that will hardly ever be encountered in real life (unless for those who tend to lurk behind people that are having conversations). The reason why a viewer will identify an OTS shot as part of a dialogue scene is that he or she has acquired knowledge of the code that defines how meaning is purveyed in motion pictures.

But let me return to the potential ambivalence of signs and offer another explanation why such a pluralism of meanings does not pose a problem for the analysis of communication forms such as television. The reason is that we are dealing not, as Umberto Eco would say, with aesthetic text such as art $(1979,261-264)$, but rather with what Roland Barthes calls mass communication $(1977,38 \& 51)$. Barthes uses the example of the advertisement image to show how verbal messages are used to reduce the potential ambivalence of images in 
popular culture. This can happen through two techniques: anchorage and relay (ibid., 38).

Anchorage is used in order to clarify what a sign denotes (ibid., 39):

The denominative function corresponds exactly to an anchorage of all the possible (denoted) meanings of the object by recourse to a nomenclature. Shown a plateful of something (in an Amieux advertisement), I may hesitate in identifying the forms and masses; the caption ('rice and tuna fish with mushrooms') helps me to choose the correct level of perception, permits me to focus not simply my gaze but also my understanding.

In Barthes' example, two redundant signs that denote the same object (one visually, one verbally) are used in order to prevent a misinterpretation of what is supposed to be denoted, thereby 'anchoring' the viewers perception. In a similar fashion, a connoted meaning can be 'relayed'. In such a case (ibid., 39-40):

...the linguistic message no longer guides identification but interpretation, constituting a kind of vice which holds the connoted meanings from proliferating, whether towards excessively individual regions (it limits, that is to say, the projective power of the image) or towards dysphoric values. An advertisement (for d'Arcy preserves) shows a few fruits scattered around a ladder; the caption ('as if from your own garden') banishes one possible signified (parsimony, the paucity of the harvest) because of its unpleasantness and orientates the reading towards a more flattering signified (the natural and personal character of fruit from a private garden); it acts here as a countertaboo, combating the disagreeable myth of the artificial usually associated with preserves.

Through a verbal statement, the ambivalence of the image is reduced, and one particular interpretation is relayed to the viewer. We could quite easily imagine, to return to the hypothetical example made earlier, that the image of the off-road vehicle was part of a car commercial. In order to assure that those looking at the image associated it with the intended connotation (the car signifying freedom), a verbal caption could relay that notion by stating "experience the freedom of the great outdoors" (or something similar). Another verbal statement could prevent the unwanted association with environmental degradation, for example by stating that the car had "the lowest $\mathrm{CO} 2$ emissions in its class". But so far these relaying signs have all been verbal. I would argue that signs in other modes (for instance visual or acoustic signs) can also relay an intended connotation. Imagine, for instance, that in 
the image the outdoor car drove along the Grand Canyon and past a large American flag while an eagle soared in the background. Even without the verbal message, the connotation of freedom would be relayed (at least in a US-American context; other viewers might connote such an image with entirely different things, since they were applying a different code). Similarly, if the commercial featured the melody (without the lyrics) of a popular song such as Free Falling by US-singer-songwriter Tom Petty, the signification of freedom would still be acoustically relayed (provided that the recipient was familiar with that song). In order to define this process of sign arrangement, Barthes introduced the term ideology, and his use of the term is much more readily compatible with the discourse analytical approach presented in Chapter 2.3 than is Norman Fairclough's interpretation of the word $(1995,17) .{ }^{23}$ According to Barthes $(1977,40)$, ideology refers to the practice of arranging signs in different modes in order to relay a certain discursive position. I would therefore argue that when analysing complex forms of mass communication such as television dramas, we need to pay particular attention to the question of how different signs interact in order to relay (i.e. disambiguate) a message, and whether that message is part of a particular discursive position. In the extreme this may mean that we are looking at the intentional promotion of the discursive position held by a particular political agent or institution. This is what I will refer to here as propaganda.

The above considerations make it less useful for the present analysis to refer to iconic, indexical and symbolic signs. Such distinctions are important to understand the various ways in which signs interact with their objects and their interpretants, but they offer little explanatory power with regard to the question of how signs function in multi-dimensional communication processes. It is therefore more useful to instead, as Barthes suggests (ibid., 34), distinguish signs according to their substance, meaning according to the dimension they

\footnotetext{
${ }^{23}$ For an interpretation of the term ideology in CDA that largely correlates with Barthes' usage of the term. see van Dijk, 2003, 24.
} 
function in. This means that we need to discern signs such as verbal signs, acoustic signs, visual signs, gestural signs, etc. This is essentially what Kress and Van Leeuwen mean when they are referring to the various modes of discourse (what Eco would call a channel; 1979, 174-176), and state "that media become modes once their principles of semiosis begin to be conceived of in more abstract ways (as 'grammars' of some kind)" (Kress et al., 2001, 22).

The mass communication medium of television drama then consists of signs in different modes, which function according to a specific socio-cultural code (just like language functions on the basis of grammar). One has to then wonder how culturally specific the code of a medium such as television entertainment is, and whether a researcher from one cultural background can decode the content of such a medium in another culture (for instance a European analysing television drama in China). I wish to argue that the code systems for visual mass entertainment may indeed possess certain culturally-specific aspects, for example when the colour white signifies death in a Chinese drama, but innocence in a Western series. However, even though the connotation of the sign in question may be different depending on the cultural context, the general rules that govern the use of such signs are the same: Even a viewer who is not familiar with the cultural meaning of the colour white in the Chinese context can still detect that he is confronted with a case of colour symbolism. Chinese television dramas of course feature an array of visual or acoustic signs that are decidedly Chinese (certain types of musical scores, background sounds such as gongs or bells, certain colours, the use of the Chinese written language, culturally specific gestures, and of course all verbal statements made in Chinese), however throughout my research I did not come across any cases in which the rules of arranging these signs deviated from those adopted in Western television entertainment. The Chinese television dramas reviewed for the present research adopted the same tools of editing, montage, choice of camera angles, camera movements, etc. in the same conventionalised way that is used in the 
Western context. In a sense, the 'vocabulary' of television drama may differ from one country to the next, but the 'grammar' is nonetheless the same. This phenomenon is perfectly in line with semiotic theory; again, we are not dealing with aesthetic text (in which case an artist may indeed re-invent codes and change the rules of how communication works; Eco, $1979,188)$, but with mass communication that aims at relaying clear and unambiguous messages. In the case of television drama entertainment, this is achieved by adopting a code that is largely international.

\subsection{A Methodology of Visual Analysis}

Combining the concepts of critical discourse analysis with the semiotic notion of the sign as the detrimental unit of communication, and applying both to the communication process that is television drama, makes it possible to resort to two 'toolboxes' at the same time in order perform a visual analysis. On the one hand, discourse analysis teaches us to identify discourse fragments (i.e. discursive statements) within communication processes, and to ask what institutional background influenced their formulation. On the other hand, a semiotic vantage point opens up the methodology of visual analysis, which breaks down multidimensional communication processes such as film and television and shows how its elements work together according to conventionalised rules to form messages (visual communication analysts do not refer to these elements as signs, but I would argue that they in fact are signs).

The question then is: what is the methodology of visual analysis. To answer this question, we need to recall that motion pictures function at various levels, all of which have to be addressed by researches: First, there is the image. Its composition relies on the following factors: 
- $\quad$ Camera angle (Faulstich, 2002, 113-121; Katz, 1991, 121-129, 159, 239-245; Mikos, 2003, 182-192; Müller, 2003, 47-48; Phillips, 2002, 72-86),

- Colour schemes (Faulstich, 2002, 147-149; Phillips, 2002, 61-64),

- Lighting (Borstnar et al., 2002, 106-109; Faulstich, 2002, 146-147; Mikos, 2003, 199-204; Phillips, 2002, 65-72),

- Mise-en-scène, i.e. the arrangement of persons and objects in the scene (Borstnar et al., 2002, 97-106; Faulstich, 2002, 144-145; Katz, 1991, 102-104; Mikos, 2003, 224-227; Phillips, 2002, 9-15, 31-51; I shall include under this heading not only stage design, but also costume design and make-up), and finally

- The acting or demeanour of the persons shown in the image (Faulstich, 2002, 100; Mikos, 2003, 155-160; Phillips, 2002, 16-31).

All of these aspects are visual signs. Next, motion pictures usually contain acoustic signs, i.e.:

- Music, either on-screen or off-screen (Borstnar et al., 2002, 126-128; Faulstich, 2002, 137-143; Katz, 1991, 101-102; Mikos, 2003, 232-235; Phillips, 2002, 152-156), and

- Sound effects and ambience sounds, again either on-screen or offscreen (Borstnar et al., 2002, 125-126; Faulstich, 2002, 136-137; Katz, 1991, 101-102; Mikos, 2003, 228-231; Phillips, 2002, 148-165).

Third, the use of verbal signs is relevant, i.e.:

- Spoken text, on-screen or off-screen (Borstnar et al., 2002, 124-125; Faulstich 2002, 131-136; Phillips 2002, 144-148, 162-165, 184-186), and

- Written language on objects in the scene, etc (Phillips, 2002, 49-51).

Finally, we need to consider the dimension of time: motion pictures consist of a series of images shown in succession. Hence, the following dynamic aspects of the product need to be taken into account:

- Camera movements (Faulstich, 2002, 121; Katz, 1991, 279-291; Mikos, 2003, 193-197; Muller, 2003, 52; Phillips, 2002, 86-92),

- Montage (Borstnar et al., 2002, 133-148; Faulstich, 2002, 122-123; Katz, 1991, 321-326; Mikos, 2003, 206-222; Phillips, 2002, 109-128), and

- Cutting frequencies (Faulstich, 2002, 124-129; Katz, 1991, 321; Korte, 1999, 86; Mikos, 2003, 215-216; Phillips, 2002, 128-135). 
Since these aspects of moving pictures are perceived visually, I shall in the following refer to them also as visual signs.

Aside from these formal methods of film-making, there are numerous additional issues that need to be addressed in the analysis of motion pictures. The most important aspects include:

- Characters (traits, appearance, background, names, actions; Faulstich, 2002, 95-112; Mikos, 2003, 161-165; Phillips, 2002, 16-31),

- References (to historical or fictional events/characters; Faulstich, 2002, 174-179; Phillips, 2002, 49-51) and intertextuality (for example literary or visual templates; Borstnar et al., 2002, 74-82; Faulstich, 2002, 57-61; Mikos, 2003, 261-268; Phillips, 2002, 208-217),

- Plot (Borstnar et al., 2002, 41, 152; Faulstich, 2002, 80-94; Katz, 1991, 145152; Mikos, 2003, 128-134; Phillips, 2002, 284-290) and story (Borstnar et al., 2002, 152; Faulstich, 2002, 25-27, 59-61; Katz, 1991, 145-152; Mikos, 2003, 128-134; Phillips, 2002, 262-264), and where applicable:

- Special effects (Faulstich, 2002, 130-131; Mikos, 2003, 237-244; Phillips, 2002, 92-94).

The relevance of all of the above factors is highly dependent on what kind of product one is analysing. For instance, in a black-and-white film colour schemes will not be of relevance, in a silent movie the function of spoken language can be ignored, and in a character-driven dialogue film special effects may not play a role. The question of which aspects of a film an analysis should focus on therefore needs to be answered on a case to case basis. What is important to realise is that in each case we need to distinguish between three types of signs when analysing moving images: the visual, the acoustic and the verbal signs. This makes analysing films and television series a particularly complex task. There are, however, a number of tools which can be applied to deal with these challenges.

First, it is important to understand how films are structured. A film tells its story as a sequence of events. A sequence is, as William Phillips explains, "a series of related consecutive scenes, perceived as a major unit of a narrative film" (Phillips, 2002, 554). The sequence therefore is a unit of story-telling. Next, a scene can be defined as "a section of a 
narrative film that gives the impression of continuous action taking place in continuous time and space" (ibid.). A scene usually ends if there is a major shift in personage, setting, time, perspective or style, whereas a sequence ends when a narrative section of a film ends (an example of how scenes work together to form sequences is provided in ibid., 106-107). Finally, each scene consists of a series of shots. A shot can be defined as "an uninterrupted strip of exposed motion-picture film or videotape that presents a subject, perhaps even a blank screen, during an uninterrupted segment of time" (ibid., 555), meaning that a shot ends when a camera perspective changes due to a cut. Television dramas are essentially constructed the same way, with the difference that the narrative is further divided into episodes.

In order to analyse motion pictures, the first step is to identify the sequences of which the motion picture consists. This is done in a sequence protocol (Borstnar et al., 2002, 131-132; Faulstich, 2002, 73-80; Korte, 1999, 82-83). A sequence protocol is a table in which the researcher lists all relevant sequences of a motion picture, numbers them, and provides information on their length, central plot points, settings, characters, audio, etc. The sequence protocol therefore gives an overview of the motion picture's general structure and provides verifiable information on details that may be lost during a simple individual screening of the picture. As an example I have provided in Figure 3 a portion of my sequence protocol to the Chinese television drama GAJZ, which is analysed in detail in Chapter 4.2. 
Figure 3: Sample Sequence Protocol from the Television Drama Series "The Commissioner 2"

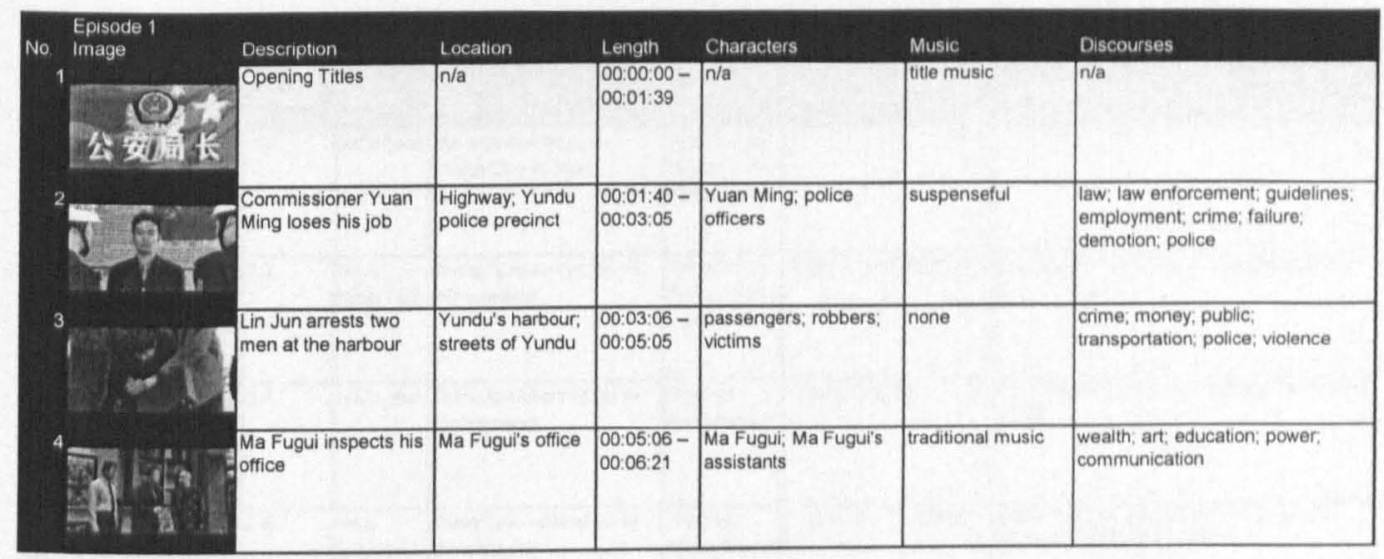

The main advantage of such a table is that it gives a quick and easy overview of the most important aspects of the film material. It may also draw attention to specific sequences (for example a particularly long sequence, one in which the music is in stark contrast to that of previous and successive sequences, etc.). A table like this should, of course, be amended according to the requirements of the particular research project. In the above example, I have included columns with information on the locations in which the sequences take place, on the characters that appear in each sequence, on the music that is used to underscore the images, and on the discourse strands that each sequence touches upon (the criteria that were used to divide each series into sequences and code them for discourse strands are explained in Section 2.6.ii). Like the methods of discourse analysis, those of visual analysis should be understood as a 'toolbox' that can be modified to suit certain research needs.

The second tool of motion picture analysis is the transcript (Korte, 1999, 80). It contains the spoken text of the picture with information on who is speaking and where (on-screen or offscreen). It may not be necessary (or useful) to transcribe an entire movie or television series, but those sections of the product which are to be analysed in detail (such as the climax of a movie, its denouement, or a sequence that strongly features a particular discourse strand) should be transcribed with scrutiny. 
Figure 4: Sample Shot Protocol from the Television Drama "The Great Han Emperor Wu"

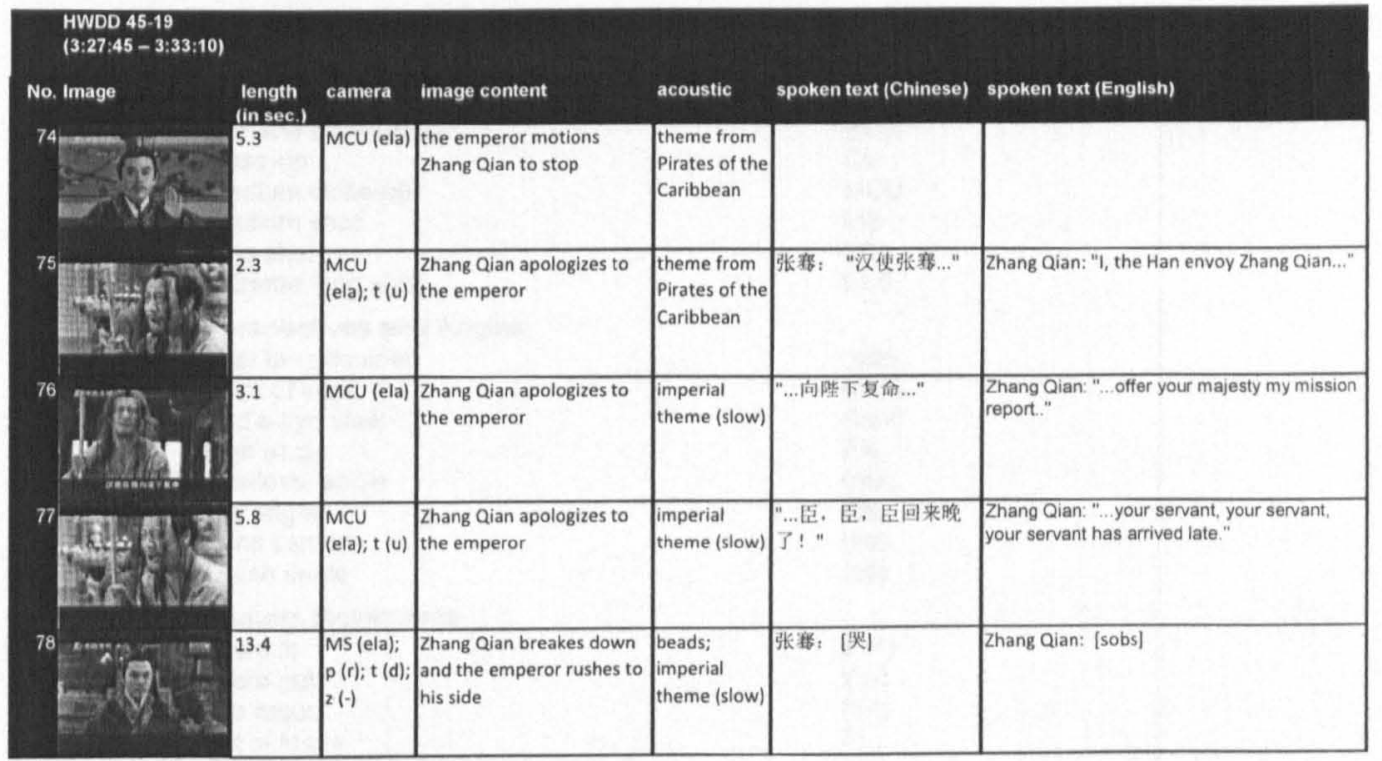

Once a specific sequence has been singled out for detailed analysis, it needs to be summarised in what is called a shot protocol (Borstnar et al., 2002, 131-132; Faulstich, 2002,66-73; Korte, 1999, 79-81). This table now provides detailed information on the acoustic, verbal, and visual signs that interact in each shot of a sequence. A shot protocol lists every single camera shot and provides short-hand information on camera movement and perspective. It also measures the exact length of every shot and maps the spoken text (in this case both the Chinese original and the English translation) in relation to the image sequence. In addition, it provides information on the music score and the acoustic signs used throughout the sequence, and shows how they coincide with the images and the spoken text. As an example, I have included part of my shot protocol to a sequence of the Chinese television drama series HWDD (episode 45, sequence 19, shots $74-78$ ) in Figure 4. For a list of the abbreviations ${ }^{24}$ used to describe camera perspectives and movements in these shot protocols, see Figure 5.

\footnotetext{
${ }^{24}$ Based on the terms and abbreviations used in Katz $(1991,159)$ and Phillips $(2002,77-81)$.
} 


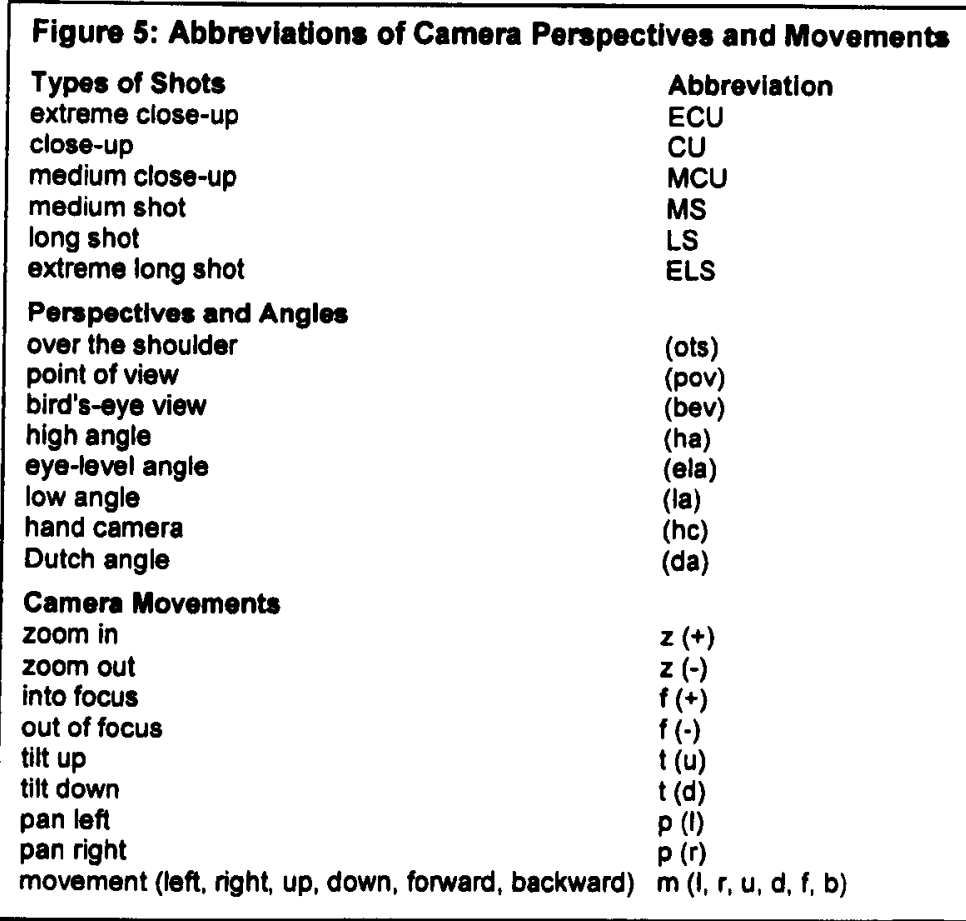

In addition to these three fundamental tools of film analysis, there are a number of methods to graphically depict certain aspects of a motion picture. One is the frequency graphic. It shows the number of cuts per minute over a certain extent of time (for instance throughout the entire motion picture, a sequence, a scene, or a section of dialogue). Such a graphic (as shown below in Figure 6) offers an overview of the general dynamics of a motion picture. Another tool is the so called shot graphic, which enables the researcher to flexibly show different aspects of a motion picture (such as camera movement, dialogue, music, content, cuts, etc.), thereby giving a detailed overview of the movies composition (for an example see Korte, 1999, 85). 
Figure 6: Frequency Graphic "Berlin: Symphony of a Great City" (Korte, 1999, 86)

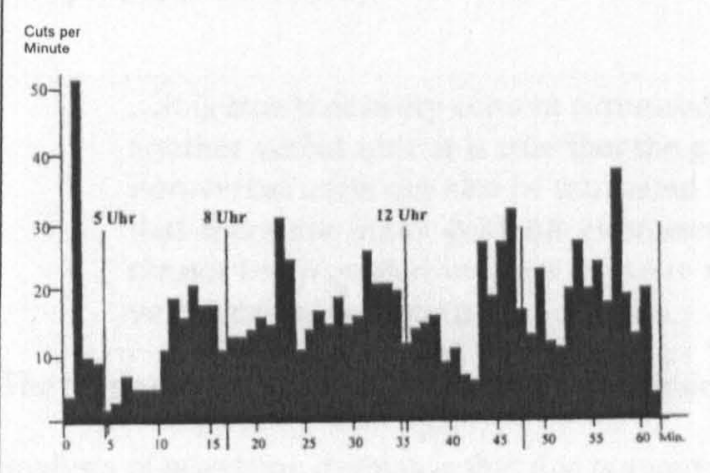

The use of protocols and graphics helps

the researcher isolate the various signs in a motion picture, and makes it possible to assess how these signs interact. Once the different signs have been identified and described, the next step is to paraphrase the discourse fragments that they form. To paraphrase signs that are non-verbal

confronts us with a practical challenge: can we use spoken and written language (as a kind of 'meta-language') in order to summarise the content of images and sounds? Or in other words: can a message that is communicated in one mode be readily translated into another mode? Umberto Eco summarises this problem when he writes that it would be "somewhat reckless to maintain that there is no difference between various types of signs" $(1979,172)$. He continues by giving an example of how difficult it can be to express verbal statements in other modes (ibid.):

It is indeed possible to express a given content both by the expression / the sun rises / and by another visual expression composed of a horizontal line, a semicircle and a series of diagonal lines radiating from the imaginary center of the semicircle. But it would seem more difficult to assert that / the sun also rises/ by means of the same visual device and it would be quite impossible to assert that / Walter Scott is the author of Waverley/ by visual means. It is possible to assert both verbally and kinesically that I am hungry (at least in Italian!) but it is impossible to assert by means of kinesic devices that «The Kritik der reinen Vernunft proves that the category of causality is an a priori form while space and time are pure intuitions" (even if Harpo Marx got remarkably near it).

Since the present research project does not attempt to transfer verbal signs into other modes (for example by drawing, whistling, or gesturing the main argument of Immanuel Kant's Kritik der reinen Vernunft), the above case need not concern us here. More problematic, however, is the fact that just like many verbal statements cannot be expressed in any mode 
other than the verbal one, there exist according to Eco also non-verbal expressions that are 'unspeakable' (ibid., 173):

...it is true that every content expressed by a verbal unit can be translated into another verbal unit; it is true that the greater part of the content expressed by non-verbal units can also be translated into verbal units; but it is likewise true that there are many contents expressed by complex non-verbal units which cannot be translated into one or more verbal units (other than by means of a very weak approximation).

The reason why this observation poses no problem when conducting a visual discourse analysis of television dramas is that Eco is again referring primarily to the 'unspeakable' qualities of aesthetic texts. It may be indeed difficult (or impossible) to articulate verbally the discursive content of the Mona Lisa, or of Beethoven's Fifth Symphony, but in the case of mass communication the content of signs stands in a disambiguated context in which we can understand (and verbally paraphrase) their message. This may require a 'weak approximation', as Eco would say, but it nonetheless makes the transfer of visual or acoustic content into verbal statements possible. Eco himself confirms this when he writes (ibid., 215216):

An iconic sign [i.e. a visual sign; author's note] is indeed a text, for its verbal equivalent (except in rare cases of considerable schematization) is not a word but a phrase or indeed a whole story; the iconic representation of a horse does not correspond to the word / horse/ but rather to a description (a black horse, standing up, or jumping, etc.), to a mention (this horse is galloping) or to some other different speech act (look, what a beautiful horse!) If inserted in a scientific text, an iconic sign can correspond to a statement of the type /all horses have four legs and such visual properties .../.

The units composing an iconic text are established - if at all - by the context. Out of context these so-called 'signs' are not signs at all, because they are neither coded nor possess any resemblance to anything. Thus insofar as it establishes the coded value of a sign, the iconic text is an act of code-making.

Barthes makes a very similar argument, when he writes that visual connotations can be difficult to phrase verbally, but that denotations are a form of syntagm, and hence "very close to speech" $(1977,52)$. Since forms of mass communication (such as television dramas) place connotative visual signs in the context of denotative signs, the formerly otherwise 
unspeakable aspect of the icons and symbols becomes naturalised: "the discontinuous connotators are connected, actualized, 'spoken' through the syntagm of the denotation, the discontinuous world of symbols plunges into the story of the denoted scene as though into a lustral bath of innocence" (ibid.). For this reason, the following analysis focuses first on identifying the multi-modal sign-functions that work within television dramas, and second on paraphrasing the discourse fragments that these sign-functions help construct.

\subsection{The Basis for the Production Analysis: Interviews with Chinese Media Experts}

Both discourse theory and semiotics teach us that the production of media content is a social as well as political process, and that in order to understand how discourses are formed, this process needs to be explored. Consequently, the main analysis of Chinese television drama content that this thesis focuses on (Chapter 4 ) is augmented by a background analysis of drama production procedures in China (Chapter 3). Since this "production analysis", to use the terminology of Marion Müller $(2003,24)$, is primarily 25 based on fourteen qualitative interviews conducted with Chinese media experts in the summer of 2007, I shall first offer an overview of the criteria which provided the foundation for the selection of interview subjects (2.5.i), the formulation of interview questions (2.5.ii), and the subsequent qualitative analysis (2.5.iii). Also, this section covers the ethical considerations that accompanied this research (2.5.iv).

\subsubsection{Selection of Interview Subjects}

The interviewees who participated in this research project were selected either as internal or external experts on the Chinese television drama market. Internal experts were recruited for their first-hand knowledge of production, distribution and broadcasting procedures. They included professionals with experience as screenwriters, directors, producers (at large state-

\footnotetext{
${ }^{25}$ In addition to interview statements, Chapter 3 further draws from primary written sources such as official government documents and statistic material were applicable.
} 
run production units and private companies, both at the central and the provincial level), editors, investors, censors and broadcasters. Experts from outside the market (i.e. academics with a research focus on Chinese television dramas) were approached for their knowledge of the general Chinese system of television drama production and broadcasting. In the majority of cases, the interviewees worked in several of these fields simultaneously (for instance when research subjects held positions as university professors, screenwriters and producers at the same time). All of the interviewees are Chinese nationals and were working in the Chinese media sector or at a Chinese university at the time of the interviews. Some had inside information to offer on the production and meaning of the TV dramas that are being analyzed in Chapter 4 of this dissertation, but that was not a necessary requirement for interviewee selection.

Once the necessary contact details of relevant experts had been gathered, the potential interviewees were contacted via email. If individuals were not available through email, it was attempted to reach them on the telephone. All persons contacted were provided with an outline of the research project. Those who agreed to participate were further asked for advice on how to contact other potential research subjects. By Mai 2007, sixteen media experts based in Shanghai, Beijing and Nanjing had been recruited. These experts were then interviewed during the subsequent two months in a total of fourteen interview sessions (interviews 13 and 14 were conducted with two interviewees each).

\subsection{Research Procedure: Structure and Questions of the Qualltative Intervlews}

Methodologically, the interviews were designed to be semi-structured and qualitative in nature ${ }^{26}$, although quantitative statements were encouraged as well. The sessions were each scheduled for one hour (giving time for at least forty-five minutes of actual interview time),

20 The interview methodology was based on Froschauer ef al. (2003, 15-41 \& 51-79), and was prepared in line with the methodological quality standards defined in Mayring (2002, 140-148). 
but were allowed to last longer, in some cases up to three hours. The location for the interview was selected by the respective research subject. ${ }^{27}$

The purpose of the interviews was threefold: To gather factual information on the workings of the television drama sector, to establish what aspects of drama production Chinese experts considered relevant, and to analyse how the interviewees talked about their area of expertise as well as the relevance of television dramas in China. If an interviewee had particular insights into the production of the television drama series that this research focuses on, then the conversation would focus on that particular theme accordingly. Each interviewee was generally asked questions ${ }^{28}$ concerning three different aspects of TV dramas:

1. The production and broadcasting process,

2. The legal framework governing the TV drama market,

3. The social impact of TV dramas in China.

However, the conversations did not necessarily proceed in this order, and the focus that was placed on the different aspects varied depending on the respective interviewee's area of expertise. This was done in order to offer the interview subjects room to fully voice their own opinions and emphasise what they themselves felt were relevant issues. For this reason, the conversations were kept as open as possible. Particularly with respect to the third set of questions (concerning the social impact of TV dramas), the purpose of the questions was not to gather factual evidence, but to establish what Chinese media experts' opinion and line of argument on the topic were.

\footnotetext{
${ }^{27}$ In the majority of cases the interviewees opted for a public setting, such as a cafe or teahouse. Otherwise the interviews were conducted in the respective research subject's office.

28 If the interviewees asked to receive more information concerning the questions that would be asked during the interview, they were provided with a broad outline of the topics under discussion as well as some sample questions.
} 
All conversations were conducted in standard Chinese, and were recorded with permission

of the interviewees. In addition, research notes were taken both during and immediately

after the conversations.

\section{5.iii Processing, Analyzing and Referencing the Interviow Material}

Once the interviews had been concluded, the recorded material and the research notes were

used to summarise each conversation in an interview protocol. The first step in creating these

protocols was to divide the interviews into narrative segments, providing a time-code for

each segment. The segments were then arranged in tables, which featured information on the

topics discussed during each of the segments, as well as paraphrases of what had been said.

Additional research notes were added in brackets. The interview protocols are presented in

Appendix 2. Throughout this thesis, they are referenced in brackets that include the

following information: the number of the interview "I\#", plus the recording time-code. For

example, the reference (I1, 0:00 - 1:00) refers to the first minute of interview number one.

In addition to the interview protocols, interview segments containing particularly

noteworthy arguments by the interviewees were transcribed. ${ }^{29}$ The transcripts attempt to

reproduce each spoken syllable, including particularities of the spoken language. ${ }^{30}$ These

\footnotetext{
${ }^{20}$ There are three reasons why only selected parts of the interviews were transcribed: First, the present research procedure does not aim at a discursive fine-analysis of the interviews, making it unnecessary to capture the entire recorded material in transcribed form; second, the amount of recorded material (a total of twenty hours) made a more expansive transliteration unfeasible; and third, in the majority of cases the production of full transcripts was found to not yield much additional information compared to the paraphrasing of the interview segments.

${ }^{30}$ The transcriptions follow the conventions presented in Froschaver et al. (2003, 223-224): Interviewees are marked as "11" through " 114 "; if more than one interviewee was present, letters were used to differentiate between them (for instance "I13a" and "I13b"). The researcher is marked as "R". Pauses are marked by periods, with each period being equivalent to one second. Nonverbal exclamations or interjections by others are presented in brackets, and exclamations are written out in cursive script: (R: laughs). Noises are presented in pointed brackets: >mobile phone<. English words are marked as follows: "boyfriend". Verbal exclamations are presented by a corresponding Chinese character in the original and by an English equivalent in the translation (such as "lify" becoming "oh"; "哪" becoming "uhu", etc). Emphases are underlined: "really". Unintelligible sections are marked by periods in brackets, one period representing a syllable, l.e. "(. . . .)" marks four unintelliglble syllables. Where the meaning of such sections can be guessed from the context or from the research notes, an approximation is presented in brackets, such as "(. . . . 的影唃)", i.e. "(influence of . . . .)".
} 
transcripts were then edited and translated into English by the author to produce the quotes used throughout this thesis. ${ }^{31}$

\subsection{Iv Ethic Guidelines for Interviow-Based Research}

All interviews were conducted and analysed in line with the ethics guidelines adopted by

the School of East Asian Studies of the University of Sheffield. No remuneration was offered for the involvement in this project, and all interviewees participated voluntarily. Prior to the interviews, all participants were informed about the project's purpose, as well as the scope of their involvement (the information leaflet for interviewees is reproduced in Appendix 2). It was explained to all participants that they were not required to discuss any matters they did not wish to talk about. It was also made clear that no information would be used in this research that the interviewees felt was subject to non-disclosure. All interviewees will remain anonymous, and the interview recordings are used only by the researcher, and solely for the purpose of this research project. The fieldwork conducted as part of this research project has been reviewed and approved by the University of Sheffield's Research Ethics Committee..$^{32}$

\subsection{The Basis for the Product Analysis: A Cross-Genre Examination of Chinese Television Drama Series}

Considering the vast amount of television drama content that is produced in China each year, this project was faced with the question of how to select representative material (2.6.i), and how to conduct the main part of this project: the visual analysis (2.6.ii).

\footnotetext{
${ }^{31}$ The transcriptions were edited into simplified quotes for two reasons: First, the public setting of most interviews and the overall non-ideal interview conditions had led to recordings of pure quality. which often made it impossible to identify each individual spoken syllable in the respective interview segments. Whereas this did not pose a problem when formulating paraphrases, it was a crucial factor in deciding not to conduct a more comprehensive linguistic fine-analysis of the material. Second, the conventions of transcribing spoken language made the interview sections umwieldy for the purpose of this analysis, while offering little additional information concerning what had been said. For these reasons, the quotes were simplified in the following ways: 1) nonverbal exclamations have largely been removed; 2) repetitions and other peculiarities of the spoken language have been deleted to produce a more readable languaga; 3) inaudible or unintelligible words in the recordings were, if possible, replaced by the likeliest approximation, derived from the context and the researcher's interview notes.

32 It should be pointed out that the British ethics guidelines adopted for this project ignore the practical issues of conducting fieldwork in China. The formal requirements on how to recruit research subjects and inform them about the research project's purpose was regarded by most interviewees as excessively official. Considering that any form of 'officialism' is viewed highly sceptically in mainland China, the ethics procedures probably hampered the fieldwork more than they contributed to it. I would argue that revisions of these guidelines are necessary to accommodate the cultural specifics in other countries. Concerning practical issues and problems of conducting fieldwork in China, see the various contributions in Heimer et al. (2005).
} 


\section{6.j The Selection of Representative Research Material}

In order to ensure that the material selected for this analysis offered representative insights into the political discourses presented in popular television series, the programmes had to fit the following seven criteria:

1. The programmes had to be television drama mini-series or telenovelas:

For this analysis, any drama that was not conceived as a serial was omitted, as were all programmes that did not classify as a drama narrative (for instance situation comedy, documentaries, or popular programmes such as game shows, etc.). The logic for this choice was that this analysis aimed at examining popular entertainment, and that drama series are the most popular entertainment format in China (see Section 2.2.iii).

2. The programmes had to have been aired on CCTV-1 or CCTV-8 during prime time: Limiting the analysis to CCTV drama programmes ensured that the series had undergone the most extensive censorship proceedings (18, 39:06 - 41:37; I12, 32:09 - 35:34) and potentially reached the largest possible audience. Programmes solely produced for and broadcasted on provincial, municipal, or satellite stations were not included in this analysis, seeing as they were tailored to more specific and hence less representative conditions (see Sections 2.2.i and 2.2.ii for a description of the various Chinese broadcasting outlets and their relevance).

3. The programmes had to have been aired between 1 January 2003 and 31 December 2005: The selection of an arbitrary recent time interval was meant to limit the number of possible programmes, and offer insights of the discourses created at one point in time.

4. The programmes had to have been among the most popular dramas aired in the respective year: 
Since the aim of this analysis was to assess the political discourses on popular Chinese TV, it had to be ensured that the drama series had indeed been well received by audiences. For this reason, only programmes were selected that had been listed among the twenty dramas with the highest audience ratings for that particular year in the respective China TV Ratings Yearbook (Wang, 2004; 2005; and 2006).33

5. The three drama series had to cover a wide range of different genres and production backgrounds:

In order to guarantee that the widest possible variety of discourse fragments was covered in this analysis, series were selected from three different genres: one historical drama, one modern teen drama, and one crime drama. Also, it was attempted to choose one programme that was entirely state-produced (a so-called 'main-melody' drama), one that was produced at least partially with state funding but did not aim to be a main-melody series, and one programme that was entirely funded and produced by private institutions. This was meant to offer insights into whether or not discourses were constructed differently depending on the institutional background of the drama series.

6. The dramas had to deal with as many political discourse strands as possible:

If various drama series equally fulfilled the criteria one through five, priority was given to the drama that most clearly and most broadly dealt with discourses of governance and its goals (see Section 2.3.v).

\footnotetext{
${ }^{33}$ The use of CSM data was owed to the fact that it is the only available statistical information on annual drama consumption. The data was not meant to provide exact quantitative information on how many viewers actually tuned in for a particular series (the questionable reliability of such data is discussed in Sections 2.2.iv and 3.4.i), but was instead used as a qualitative indication of whether a programme had been registered among the most watched series of a certain year.
} 
7. The drama series had to be available on DVD:

Programmes were only considered for analysis if legal working DVD version could be purchased from an official distributor. This was meant to ensure the technical feasibility of the visual analysis and the highest possible quality of the visual material.

After establishing these criteria, all available television dramas that ranked among the twenty most popular series aired on CCTV in 2003, 2004, and 2005 were purchased and testscreened. The following three series were then chosen and prepared for the detailed analysis: the epic historical drama HWDD, the main-melody crime drama GAJZ, and the teen drama NCNM.

\section{6.ll Coding the Material for Polltical Discourses}

As a first step in my analysis, the visual material had to be prepared for detailed study. In order to ensure a systematic examination of the material, the three television drama series mentioned above were therefore each divided into sequences, and these sequences were then summarised in protocol tables (see DVD in the back of the appendix volume). These sequence protocols were arranged in a way that offered an easy overview of the personage presented in each sequence, the type of music used to underscore the drama, and the locations in which the individual sequences were set. Time codes are provided to simplify the process of finding certain sections of the visual material on the respective DVDs. ${ }^{34}$

Next, each sequence was coded with a variety of key words, relating to the discourse strands discussed in Section 2.3.v. To this effect, an evolutionary coding procedure was used (see Figure 7), based on the suggestions in Mayring $(2002,120)$. This resulted in the following working steps:

\footnotetext{
34 Time codes may vary slightly, depending on the hardware and software used to review the DVDs; for this analysis, a computer DVD drive and the Cyberlink DVD software were used.
} 
1. A preliminary list of key words was drafted. This list was derived from the central discourse strands discussed in Section 2.3.v and was based on theoretical considerations of how each of the main discourse strands could be divided into further subcategories.

2. The visual material was then screened with these theoretical concepts in mind, starting with the series NCNM. Each sequence was associated with the key words relevant to the respective context, and additional terms were created as new topics arose in the narrative. In this sense, the list of coding categories was significantly expanded, based on practical considerations.

3. After the first cycle of coding had been completed, the original list of key words was revised to incorporate the new words. In some cases, words were 'collapsed' into one category, in others a single key word was divided into several codes..$^{35}$

4. This process of expanding and collapsing the coding categories was then repeated with the next drama series (GAJZ), again followed by a revision. After the third series had been coded in this manner, the table of key words was reworked one last time to yield the final list of coding categories.

Note how these codes encompass a wide variety of issues, most of which are not analysed in this research project. There were two reasons for adopting this very wide range of coding categories: First, to ensure that the juxtaposition and interaction of various discourse strands could later be assessed (for example when issues of security were repeatedly crossed with

\footnotetext{
${ }^{35}$ After the first coding cycle, an attempt was made to create a category tree that clearly attributed each of the codes with one discourse strand. This initial attempt was abandoned, however, since many of the codes did not clearly fit into one single discourse calegory. For instance, sequences coded with the term "hierarchy" in some cases dealt with welfare issues (for example in NCNM, were numerous sequences showed hierarchical family relations or the relations between a superior and a subordinate in business ventures). in other cases they were related to security issues (for example dealing with the relation between a superior and a subordinate in the police force in GAJZ and NCNM, or with military (urisdiction in HWDD), and in yet other cases they could be associated with issues of governance (for example relating to ruler-minister relations in HWDD, or the relation between the police commissioner and the party secretary in GAJZ). In order to avoid limiting the explanatory power of the codes, it was therefore decided to not forcefully relate the various categories at this stage, but to rather focus on the discursive function of the respective codes during the final qualitative analysis instead (for example by examining how hierarchical relations are used to make statements about police work and public security issues, and to separately assess how such statements are linked with other issues, such as legitimacy or identity).
} 
issues of nationalism, or when issues of ruler-subject relations appeared in connection with welfare issues such as poverty); second, to lay the basic groundwork for further research into other discourses presented in these series (for example concerning discourse strands such as gender, consumption, technological development, etc.).

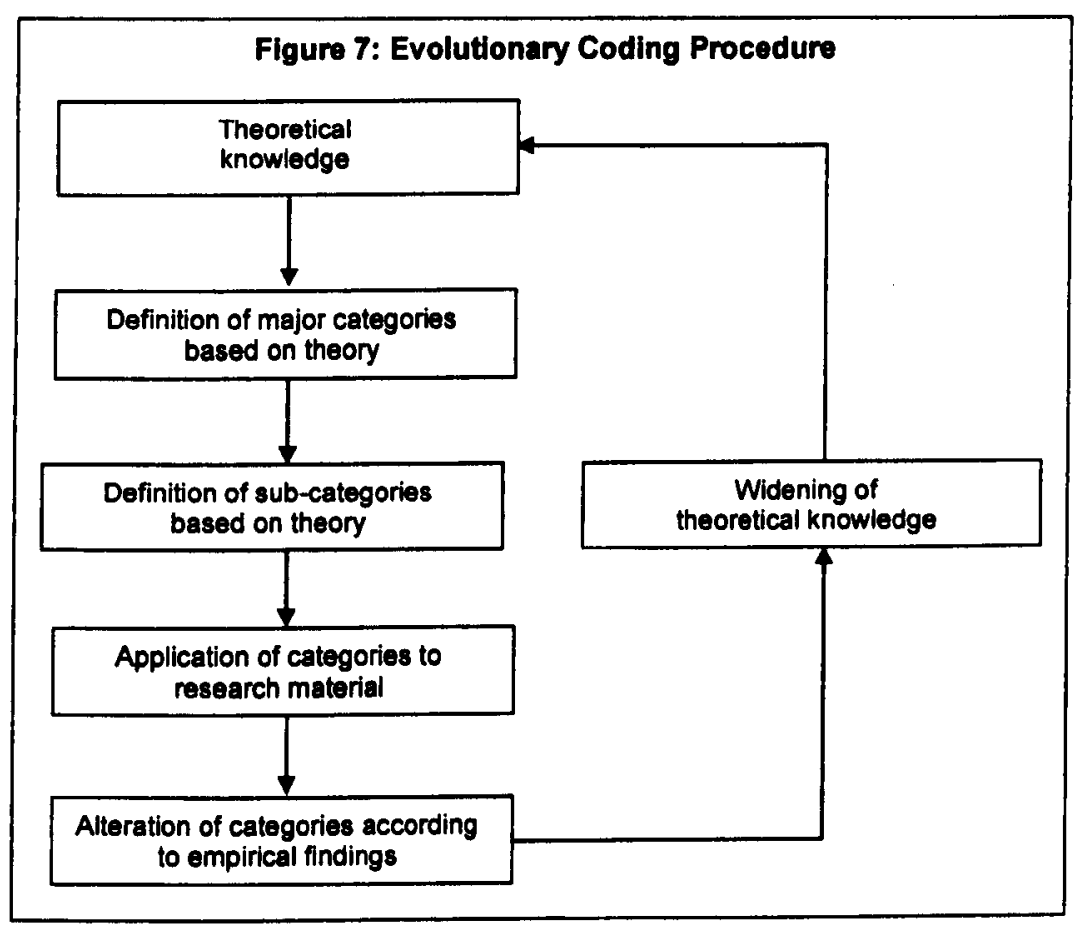

Once the sequence protocols of all three series had been completed, the actual fine-analysis started. The sequences that offered the most information on the main discourse strands were selected, and detailed shot protocols of each were devised. The criteria for selecting certain sequences as starting points for the fine-analysis were the following:

- Primary criterion: The discourse strand under scrutiny had to be prominently featured in the sequence. 
- Secondary criterion 1: In cases where a large number of sequences fulfilled the main criterion, priority was given to those with central importance either to the main narrative, to one of the narrative arcs of the programme, or to the characterization of a main character.

- Secondary criterion 2: Sequences that featured a wide variety of signs (i.e. visual, acoustic and verbal) were given priority over those that functioned primarily in one mode (such as dialogue scenes that focused only on verbal signs, and included only few visual signs).

The information on the discursive statements made in these sequences and on the way they are made was then compared to other sequences that dealt with the same issues. If additional information could be gained from subjecting these other sequences to a fine-analysis, then a shot protocol was devised for that respective sequence as well. The collected findings of this research procedure are presented in the main chapter of this thesis (Chapter 4), and all relevant protocols are provided on the appendix DVD. ${ }^{36}$

\footnotetext{
30 The system used to reference the visual material functions as follows: first, the drama series is stated in abbreviated form; next, the episode number follows; third, the sequence number is provided, and finally (where applicable) the shot number is added. For example: NCNM 01-02-03 refers to the third shot in the second sequence of the first episode in Nancai Numeo.
} 


\subsection{Summary: Towards an Integrated Methodology of Critical Visual Discourse Analysis}

It should be clear from the discussion above that recent research projects have indeed

covered a wide area of communication practices that relate to mass-communicated media

content. This includes the analysis of TV and television dramas in the PRC. However, such

analyses are still rare in the field of Chinese Studies, and certainly in the Western social

sciences; also, past studies lack a consistent methodological approach. Considering the

central role that television drama plays in Chinese society, this is a gap that needs to be filled.

In order to do so, a comprehensive theoretical and methodological framework is required that addresses the question of what a television drama's content is, what institutional background led to its creation, and how the process of producing and disseminating such content is relevant to wider social and political practices. For this purpose I am proposing a qualitative approach that combines critical discourse theory with semiotics. A list of the major technical terms of such an approach is provided in Figure 8 below.

Figure 8: List of Technical Terms and their Definition

\begin{tabular}{|c|c|c|}
\hline Term & Definition & Source \\
\hline Aesthetic Text & $\begin{array}{l}\text { A message coded in such a way that it intentionally } \\
\text { leaves ambiguities with regards to the contents that the } \\
\text { signs denote and connote. }\end{array}$ & Eco 1979, pp.261-264 \\
\hline Anchorage & $\begin{array}{l}\text { Two or more signs in different modes used to } \\
\text { disambiguate a denotation. }\end{array}$ & Barthes 1977, p.38 \\
\hline Code & $\begin{array}{l}\text { A system of correlational rules linking signs to content, } \\
\text { based on social and cultural conventions. }\end{array}$ & $\begin{array}{l}\text { Eco 1979, pp.33-38 } \\
\text { \& } 191-199\end{array}$ \\
\hline Communication & $\begin{array}{l}\text { The practice of coding a message and transmitting it from } \\
\text { a source to a destination, where it is decoded and } \\
\text { interpreted. }\end{array}$ & Eco 1979, p.33 \\
\hline Connotation & $\begin{array}{l}\text { A complex sign-function in which a sign stands for the } \\
\text { content of another sign-function. }\end{array}$ & $\begin{array}{l}\text { Barthes 1977, p. 37 } \\
\text { Eco 1979, pp.55-56 }\end{array}$ \\
\hline $\begin{array}{l}\text { Counter-Hegemonic } \\
\text { Discourse }\end{array}$ & $\begin{array}{l}\text { Communication practices that contest the hegemonic } \\
\text { discourse in a society and attempt to replace it with an } \\
\text { alternative world-view. }\end{array}$ & Jäger 2004, pp.129-130 \\
\hline Denotation & $\begin{array}{l}\text { A simple sign-function in which a sign stands for a } \\
\text { content. }\end{array}$ & $\begin{array}{l}\text { Barthes 1977, p.37; } \\
\text { Eco 1979, pp. } 54-57\end{array}$ \\
\hline $\begin{array}{l}\text { Diachronic } \\
\text { Discourse Analysis }\end{array}$ & The analysis of the changes in discourse over time. & Jäger 2004, p.171 \\
\hline Discourse & $\begin{array}{l}\text { Communication practices that construct our knowledge of } \\
\text { reality over time. }\end{array}$ & $\begin{array}{l}\text { Foucault 2005, pp.30 } \\
\& 54 ; \\
\text { Jager } 2004, \text { pp. } 129 \\
\& 184 ; \\
\text { Potter } 2005, \text { p. } 86\end{array}$ \\
\hline
\end{tabular}




\begin{tabular}{|c|c|c|}
\hline $\begin{array}{l}\text { Discourse } \\
\text { Fragment }\end{array}$ & $\begin{array}{l}\text { A discursive statement, i.e. a statement that constitutes a } \\
\text { claim to truth. A discourse fragment is part of one or more } \\
\text { discourse strands and is expressed in a certain discourse } \\
\text { layer. It can be used to express a discursive position. }\end{array}$ & $\begin{array}{l}\text { Foucault 2005, p.30-31 } \\
\text { Jäger 2004, pp.166-168 }\end{array}$ \\
\hline Discourse Layer & $\begin{array}{l}\text { The institutional level or setting in which discourse takes } \\
\text { place, for example the academic layer, the political layer, } \\
\text { the media layer, the private layer, etc. }\end{array}$ & Jäger 2004, pp.163-165 \\
\hline Discourse Strand & $\begin{array}{l}\text { A certain discourse topic, for example globalization, } \\
\text { migration, crime, etc. }\end{array}$ & Jager 2004, pp.1630-163 \\
\hline Dlscursive Position & $\begin{array}{l}\text { Individual perspective of what is true, formulated through } \\
\text { discourse fragments. }\end{array}$ & Jager 2004, p.163 \\
\hline $\begin{array}{l}\text { Hegemonic } \\
\text { Discourse }\end{array}$ & $\begin{array}{l}\text { Communication practices that reflect the dominant world- } \\
\text { view of society, kept stable through power relations. }\end{array}$ & $\begin{array}{l}\text { Jager 2004, pp.129-130; } \\
\text { MacDonald 2003, p.32 }\end{array}$ \\
\hline Icon & A sign that bears a similarity to its object. & $\begin{array}{l}\text { Eco 1979, p.178; } \\
\text { Peirce 1991b, pp.239-240 }\end{array}$ \\
\hline Ideology & $\begin{array}{l}\text { The practice of arranging multi-modal signs in order to } \\
\text { relay a certain discursive position. }\end{array}$ & Barthes 1977, p.40 \\
\hline Index & A sign that is the consequence of its object. & $\begin{array}{l}\text { Eco 1979, p.178; } \\
\text { Peirce 1991b, pp.239-240 }\end{array}$ \\
\hline $\begin{array}{l}\text { Mass } \\
\text { Communication }\end{array}$ & $\begin{array}{l}\text { A process of communication in which methods of } \\
\text { anchorage and relay are used to disambiguate } \\
\text { messages, thereby guiding recipients to one pre-selected } \\
\text { interpretation of that message. }\end{array}$ & Barthes 1977, p.38\&51 \\
\hline Message & Content coded through signs. & Eco 1979, p.33 \\
\hline Mode & $\begin{array}{l}\text { Levels at which signs can function, such as the visual, } \\
\text { acoustic, or verbal mode. Sometimes also referred to as } \\
\text { a channel. }\end{array}$ & $\begin{array}{l}\text { Barthes 1977, p.34; } \\
\text { Eco 1979, pp. 174-176; } \\
\text { Kress \& Van Leeuwen } \\
\text { 2001, p.22 }\end{array}$ \\
\hline Propaganda & $\begin{array}{l}\text { Discourse fragments constructed through ideology with } \\
\text { the intent to promote the discursive position of a } \\
\text { particular political agent or institution. }\end{array}$ & $\begin{array}{l}\text { Indirectly: Barthes } 1977 \text {. } \\
\text { p.40 }\end{array}$ \\
\hline Relay & $\begin{array}{l}\text { Two or more signs in different modes used to } \\
\text { disambiguate a connotation. }\end{array}$ & Barthes 1977, p.38 \\
\hline Scene & $\begin{array}{l}\text { A section of a narrative film that gives the impression of } \\
\text { continuous action taking place in continuous time and } \\
\text { space. }\end{array}$ & Phillips 2002. p.554 \\
\hline Sequence & $\begin{array}{l}\text { A series of related consecutive scenes, perceived as a } \\
\text { major unit of a narrative film. }\end{array}$ & Phillips 2002, p.554 \\
\hline Shot & $\begin{array}{l}\text { An uninterrupted strip of exposed motion-picture film or } \\
\text { videotape that presents a subject, perhaps even a blank } \\
\text { screen, during an uninterrupled segment of time. }\end{array}$ & Phillips 2002, p.555 \\
\hline Sign & $\begin{array}{l}\text { Anything that, on the grounds of a previously established } \\
\text { social convention, can be taken as something (referent) } \\
\text { standing for something else (object) to someone else } \\
\text { (interpretant). }\end{array}$ & $\begin{array}{l}\text { Eco 1979, p.16; } \\
\text { Peirce 1991b, p.239 }\end{array}$ \\
\hline SIgn-Function & $\begin{array}{l}\text { The correlation between a sign and a content based on a } \\
\text { code. }\end{array}$ & Eco 1979, p.191 \\
\hline $\begin{array}{l}\text { Synchronic } \\
\text { Discourse Analysis }\end{array}$ & The analysis of discourse at one point in time & Jager 2004, p.171 \\
\hline Symbol & $\begin{array}{l}\text { A sign that depends on its interpretant and has no } \\
\text { relation to its object. }\end{array}$ & $\begin{array}{l}\text { Eco 1979, p.178; } \\
\text { Peirce 1991b, pp.239-240 }\end{array}$ \\
\hline
\end{tabular}

The critical discourse analytical aspects of this approach allow us to view television dramas

as a type of communication practice, and explain how such practices not only reflect what is

commonly accepted to be true concerning different issues throughout society, but also how

these practices in turn reinforce and possibly restructure such truths. A critical discourse 
analytical approach also provides a methodological framework: it enables us to isolate discursive statements (so called 'discourse fragments'), and inquire how various forces (agents and structures) contributed to their formulation.

By treating television dramas as communication practices, we are further able to integrate into the critical discourse analytical approach the theory and methodology of semiotics. We can then argue that discourse fragments consist of signs, which denote and connote certain content. In mass communication products such as television dramas, these signs are arranged in various modes (visual, acoustic, and verbal) to relay precise messages to the viewers. Since semiotic theory stands in close proximity to analyses of visual materials, the integration of semiotics into discourse theory allows us to utilise a wide range of tools devised for the purpose of motion picture analysis, such as sequence protocols, shot protocols and frequency graphics. This makes it possible to identify the various different signs that function within a television drama series, and interpret the discursive statements they make.

The present analysis follows this logic. It first examines within which institutional setting television dramas are created in China, and what factors influence the production of the discourses communicated through such dramas (Chapter 3). Next, it analyses three specific popular Chinese television drama series that were aired between 2003 and 2005 in order to identify what discursive statements these programmes make concerning the two political discourse strands of governance and security (Chapter 4). 


\section{Factors in the Creation of Chinese TV Dramas}

In order to offer a coherent analysis of Chinese television drama discourse strands, the question needs to first be answered how various factors influence the production process of such discursive themes. The following chapter therefore analyses the personal and institutional factors that play a role in the Chinese TV drama sector. The analysis is based on qualitative interviews conducted with Chinese media experts in 2007 (for details on the process of selecting and interviewing the research subject, as well as on the methods used to analyse the interviews, see section 2.5 ), and is divided into eight sections. The first five of these sections focus primarily on the interviewees' factual knowledge of drama production procedures and the rules that govern the TV drama market: Section 3.1 examines the institutions (production companies and units), and the financial factors (investors, budget considerations) that play a role during the production process of Chinese television dramas. The subsequent Section 3.2 covers the evolution of TV dramas from story idea to final product and introduces the personnel involved in the relevant decision-making procedures of production (producers, directors, editors, etc.). Section 3.3 focuses on the distribution and broadcasting of TV dramas. Section 3.4 concentrates on TV drama audiences in China, establishing to what degree audience measurements, viewing habits, and viewer feedbacks influence the production of television series. Section 3.5 then deals with issues of censorship, introducing the relevant state and party institutions as well as the policies that regulate the television drama market. The final two sections focus on the perceptions of the research subjects. They examine the way in which Chinese media experts describe the functions of TV (Section 3.6) and the potential influences of television on Chinese viewers (Section 3.7). The aim of this last part is to explore what explanations and justifications are used within the domestic system to describe the relevance of TV dramas, the role of censorship and the importance of market-economic mechanisms, and to establish to what extent such 
conceptions influence the way in which TV dramas are created and distributed. Finally, Section 3.8 offers a summary of the research results.

\subsection{Production Companies and Investors}

The first factor that is relevant to the analysis of Chinese television drama discourses is how such discourses are created. This process of creating television dramas is most commonly initiated "top-down", starting with a production company $(13,13: 42-17: 43) .{ }^{37}$ The production company charges a creative producer with the creation of a marketable TV drama, thereby setting in motion the production process (ibid.). Before examining the numerous phases of this process, it is worth taking a look at the types of production units that can initiate a drama production and the institutions that offer funding for such projects.

\subsection{State-Owned Production Companies}

In general, production companies fall into either of two categories (14, 05:19 - 08:43): stateowned (guoyou 国有) or non-governmental (minjian 民间). Despite the increased liberalisation of the TV drama production market, the number of state units that posses the government's approval to produce TV content is still substantial: According to one interviewee (12, 50:47 52:11), approximately 100 central state agencies are in possession of a general production permit (xukezheng 许可证). Such units not only include professional production companies, for example the China Teleplay Production Centre (Zhongguo dianshiju zhizuo zhongxin 中国出 视剧制作中心), CCTV or the China Film Group Corporation (Zhongguo dianshi jituan gongsi ip 国电影集团公司)，but also numerous production units of local or provincial TV stations (difang dianshitai dianshiju zhizuo zhongxin 地方电视台电视剧制作中心; 12, 49:09 - 50:46). In addition, various state agencies outside of the production sector also possess such a permit.

\footnotetext{
${ }^{37}$ The alternative is the "bottom-up" approach, during which a writer with a story idea will attempt to sell his or her script to a production unit $(13,00: 49-03: 05)$. However, such approaches are risky for the screenwriters and therefore not too common: If the screenwriter is not part of a production company or unit, he or she may face substantial difficulties when trying to promote an idea. Personal and family ties play a paramount role in this respect, and one of the interviewee emphasised this by calling China an "interpersonal society" (renxing shehui $\lambda$ 性社会): Without personal contacts (guanxi 关系) or the backing of a famous and influential screenwriter, a story idea is unlikely to be considered by Chinese producers $(13,04: 46-05: 21)$.
} 
Examples include academic institutions such as the CUC (113, 65:33 - 71:07)), central

ministries such as the Ministry of Public Security (Zhonghua renmin gongheguo gonganbu 中华 人民共和国公安部; ibid.)，as well as several groups of the armed forces, such as the Army， Air Force and Navy $(13,52: 16-56: 26) .{ }^{38}$ Formerly (14, 05:19 - 08:43), the various production units used to be closely linked to their respective superior agency and were required to disseminate the propaganda content that these agencies devised. Today, however, most production units, particularly the professional production companies, operate independently (duli 独立) on the TV drama market and are increasingly profit-oriented. ${ }^{39}$ According to the interviewees, the period in PRC history during which state-owned enterprises were required to produce content merely for propaganda purposes and without consideration of potential revenues are over $(12,18: 05-20: 42 ; 16,12: 58-15: 20)$. However, state-owned units are still influenced by government and party agendas $(13,52: 16-56: 26 ; 19,37: 02-38: 21)$, which means that education and propaganda feature more prominently into their creative process of TV drama production than is the case with non-governmental companies.

\subsection{Non-Governmental Production Companles and the Rlsks of Drama Production}

An increasing competition for the state-owned production units comes from the nongovernmental production companies $(14,05: 19-08: 43) .{ }^{40}$ Since the start of the market economic reforms in China, the number of non-governmental production units has steadily increased over the past years: According to one interviewee, eighty per cent of all Chinese production companies were non-governmental in 2006 (ibid.). In contrast to the state-owned institutions, propaganda and education are rarely motives of the non-governmental actors

\footnotetext{
${ }^{38}$ For more examples of institutions that possess a production permit, see Li (2007, 80-85); as well as Lu et al. $(2006,21)$.

${ }^{39}$ For example, the production centres of most state-run TV stations (including CCTV and the provincial broadcasters) are required to sell their products on the open market like any private company; they are not automatically guaranteed that their host company will buy and air the $\mathrm{N}$ dramas that they have produced $(12$. 18:05 - 20:42).

${ }^{40}$ The term non-governmental includes both private (siren 私人) and stock-owned (gufen 投仍) companies. For an overview of the non-governmental production sector $300 \mathrm{Yu}$ (2005).
} 
(I12, $01: 54$ - 03:04). Private and stock-owned production companies do not have to adhere to explicit ideological agendas, and can therefore produce a wider range of TV dramas (ibid.).

Most large non-governmental companies are organised as professional enterprises with a long-term involvement in the market, often continuously producing TV dramas of various genres (I12, 01:54 - 03:04). In order to generate profits, they will attempt to produce TV dramas that have a high appeal to viewers while simultaneously being as uncontroversial as possible; or as one interviewee put it: "maximizing profit while minimizing political risk" (I3, 47:07 - 48:36).

In addition to the large non-governmental production companies, there are also smaller enterprises that only work on one particular project. These small firms, which one interviewee referred to as "guerrilla" companies (13,06:33 - 08:43), should not be confused with independent or avant-garde production companies in western countries: their intention is not to produce art or criticise society, but merely to generate profits with one isolated, specific production idea (13, 08:44 - 09:07). They will subsequently attempt to secure the largest possible amount of investments while simultaneously lowering their expenses drastically. In many cases, these small production companies do not expect their programmes to be aired $(I 3,10: 57-13: 41)$. The conditions in the Chinese TV drama market have created a moral hazard, in some cases prompting such small firms to deliberately produce TV dramas of sub-standard quality (ibid.). The companies will then keep the large bulk of investments for themselves, regardless of the final products fate; this is currently a common practice in China $(12,46: 57$ - 49:08; $13,10: 57$ - 13:41).

Due to the dubious profit-making strategies of smaller enterprises, the large bulk of Chinese drama productions (approximately seventy per cent according to one interviewee) is never aired (12, 45:39 - 46:56). This makes TV drama production (and the investment into the sector) a risky business (ibid.). The situation is further aggravated by nepotism, personal ties, and 
unprofessional management techniques throughout the production sector (both with respect to non-governmental and state-owned companies; 13, 106:41 - 109:38), but also by unethical behaviour on the part of reputable directors and producers (who will at times take large amounts of money for their involvement in a project, without then fulfilling their end of the agreement) ${ }^{41}$. In addition, investment speculations put an extra strain on the TV drama production sector: many private financiers will invest their funds into productions of one particularly popular TV drama genre (for example swordsmen dramas or crime series), hoping to make quick profits from a temporary trend (I2, 46:57 - 49:08). Such speculations may create an investment bubble; once over-production of that particular genre has caused the market to be saturated, the investment bubble will burst, leaving production companies unable to sell their products (ibid.).42

\subsection{Budget Items}

The production costs for Chinese television dramas vary widely, depending on the genre, the length of the drama, and the quality of the production (12, 7:25 - 9:06; I14, 53:41 - 56:03): One episode will usually cost between $300,000 \mathrm{RMB}$ (for dramas set in modern-day China) and $600,000 \mathrm{RMB}$ (for elaborate historical series), but extremely cheap productions (80,000 RMB per episode) and very expensive productions (1,000,000 RMB per episode) also occur ( 13 , 23:44 - 25:24). A drama series with twenty episodes will normally cost between $6,000,000$ RMB and 20,000,000 RMB (I1, 28:27 - 28:57; I2, 07:25 - 09:06).

A large budget item is the salary of actors (I4, 44:43 - 46:06): Famous stars demand between 100,000 and 150,000 RMB per episode (i.e. up to 3,000,000 RMB for a twenty episode drama), second rate stars are paid 80,000 to $100,000 \mathrm{RMB}$ (i.e. up to $2,000,000 \mathrm{RMB}$ for a twenty

\footnotetext{
${ }^{41}$ One interviewee gave the example of a famous, successful producer, who was recently able to secure investments based solely on an investment proposal, without having to produce a story outline, script or actual product. The producer embezzled a total of 180 million RMB from his investors and fled Chine with the money in $2006(14,18: 42-20: 47)$.

${ }^{42}$ Some interviewees justified government intervention into the $\mathrm{N}$ drama market with this argument (for example 14, 37:32 - 43:28 and 15: 80:42 - 82:51); according to the interviewees, the state has to regulate the genres that can be produced at any given time in order to prevent investment speculations. This issue is discussed below in the chapter on censorship (3.5.).
} 
episode drama), third-level stars receive about 50,000 to 60,000 RMB (i.e. up to $1,200,000$ RMB for a twenty episode drama), and little known actors are paid 5,000 to 20,000 RMB per episode (i.e. between 100,000 and 400,000 RMB for the entire production). These numbers are particularly high when considering the salaries of some of the other production crew members: a director receives between 10,000 and 100,000 RMB per episode (i.e. between 200,000 and 2,000,000 RMB for a twenty episode production; 12, 66:31 - 72:59), a screenwriter approximately 4,000 to $15,000 \mathrm{RMB}$ per episode $(80,000$ to $300,000 \mathrm{RMB}$ for a twenty episode script; $18,52: 10$ - 53:44).

\subsection{Iv Investors and their Motives}

The numerous risk factors in the Chinese drama sector prevent most production companies and producers from financing a drama solely with their own resources, or with the funds of one individual investor. It is technically possible to apply for a bank loan (yinhang daikuan 银 行贷款), but considering the high-risk environment that production companies face, most banks will only grant such loans to large, state-owned production units; non-governmental companies will not normally receive such loans (I4, 29:15 - 31:05; 110, 39:29 - 40:56). Generally, these enterprises will instead attempt to spread the investment burden and risks across several partner companies (11, 02:28 - 03:37). Potential investors can be divided on one hand into state and non-state actors, on the other hand into institutions from either within the media sector (hangnei 行内) or from outside that sector (hangwai 行外). Such a differentiation leads to four types of investors, which I have listed in Figure 9 (based on the interviews conducted in the summer 2007). 
Figure 9: Types of Investors for TV Drama Series

\begin{tabular}{|c|c|c|}
\hline & State & Non-State \\
\hline Media & $\begin{array}{l}\text { Category: } \\
\text { State-owned television stations, } \\
\text { state-owned production companies } \\
\text { Examples: } \\
\text { CCTV, Jiangsu TV, China Teleplay } \\
\text { Production Centre, etc. } \\
\text { Motives: } \\
\text { - Profits from production sales } \\
\text { - Securing future broadcasting rights } \\
\text { - Didactic or propaganda intentions }\end{array}$ & $\begin{array}{l}\text { Category: } \\
\text { Private production companies, } \\
\text { private distributors } \\
\text { Example: } \\
\text { Beijing Xingguang International (北大星光 } \\
\text { 集团有限公司; main production unit behind } \\
\text { the TV drama NCNM) } \\
\text { Motives: } \\
\text { - Profits from production sales } \\
\text { - Distribution rights or copy-rights }\end{array}$ \\
\hline Non-Media & $\begin{array}{l}\text { Category: } \\
\text { Ministries or state agencies } \\
\text { Examples: } \\
\text { Ministry of Public Security, Army, Air } \\
\text { Force, etc. } \\
\text { Motives: } \\
\text { - Profits from production sales } \\
\text { - Didactic or propaganda intentions }\end{array}$ & $\begin{array}{l}\text { Category: } \\
\text { Commercial enterprises } \\
\text { Examples: } \\
\text { Construction companies, computer } \\
\text { companies, telecommunication providers, } \\
\text { etc. } \\
\text { Motives: } \\
\text { - Profits from production sales } \\
\text { - Product placement opportunities }\end{array}$ \\
\hline
\end{tabular}

In most cases, the various investors will be professionally involved in the television sector and will pool their resources with the main production unit $(114,31: 15$ - 32:59). For example, some investors may grant the main production company the rights to use their production permit; others (for example TV stations or distribution companies) may support the marketing effort or assure broadcasting (I10, 72:21 - 73:45). Such collaboration will usually take place at the domestic level; some production firms may decide to cooperate with enterprises or individuals from outside the mainland, but this will usually not extend further than involving famous directors, producers or actors from Hong Kong, Taiwan or possibly from neighbouring East Asian countries (such as South Korea; I2, $02: 28$ - 03:35). Generally, the bureaucracy involved in more extensive international cooperation outweighs the benefits, and was described by one producer as excessively "annoying" (mafan 麻烦; ibid.).

Investors do not always contribute to the actual production process with professional knowledge or contacts. Most often, the involvement of investors will be purely financial in nature, particularly if the financiers are not themselves part of the media sector (I4, 23:19 - 
25:51). Such investors receive a share of the final profits, or (in the case of certain consumer product enterprises) are allowed to place their product in the TV drama for promotion purposes (I2, 16:37 - 18:04). In return, investors offer financial support, supply the production team with equipment (mobile phones, computers, etc.; 18, 23:10 - 24:29), or allow the use of their office space as a filming set (112, 27:28 - 28:49).

The main production unit defines the responsibilities and decision-making privileges of each investor and codifies the rights and responsibilities in individual contracts ${ }^{43}$ (I2, 14:08 - 15:39). A larger financial involvement will naturally be accompanied by more decision-making powers (15, 37:21 - 39:42). In general, however, investors have little to no influence on the genre or content of the TV drama being produced (I1, 27:33 - 28:26).

Exceptions are state investors, who usually also have requirements concerning the TV drama's content.44 This is particularly the case if the production units are themselves stateowned: Despite the official independence of production units such as CCTV's China Teleplay Production Centre, they will de-facto still produce propaganda content for their superordinated TV station $(15,41: 23-43: 20)$. The interests of TV stations and their production units are not as clearly separated as the market economic system would demand $(13,37: 13-39: 20)$. This is due in part to the overlapping of personnel and responsibilities (ibid.; I3, 106:41 - 109:38), in part to the fact that the production units (whether at the central, provincial, or municipal level) continue to financially depend on the host TV stations..$^{45}$

\footnotetext{
${ }^{43}$ Although contractual agreements are becoming more common in China, many production companies still prefer to base their business ventures on personal bonds. As one interviewee explained (14, 20:48 - 23:18), many businesses are prejudiced towards legal contracts: the argument is that a contract with a trustworthy partner is unnecessary (since such a partner will not attempt to betray the agreement), and that a contract with an untrustworthy partner is useless (because such a partner will always find a way to circumvent the agreement). 44 A good example is the crime drama series GAJZ, which is analysed in Chapler 4.2: the production was initiated and in part financed by the Ministry of Public Security (Zhonghua renmin gongheguo gonganbu 中华人民抽利政忩 安部). The ministry's motivation was to improve the image of the police. It therefore had very clear propaganda (xuanchuan 宜传) guidelines that needed to be fulfilled (113, 65:33 - 71:07).

${ }^{43}$ One interviewee gave the example of a representative municipal TV station and explained how that station pays 10 million RMB to its nominally independent production unit every year, this amounts to fifteen per cent of that unit's annual production expenses $(12,21: 46-22: 45)$.
} 


\subsection{Planning and Producing a TV Drama}

Whether the production of a television drama is initiated by a state-owned institution or by a non-governmental company, the actual procedure of developing a story and producing a drama are generally the same (I4, 14:55 - 16:17). Since Chinese TV dramas are conceived as a closed story told in numerous episodes, similar to the Latin-American 'telenovelas', a standard format for a Chinese drama series has developed: the programmes usually include twenty episodes, each approximately forty-two minutes long (see Section 2.2.iii). This sets Chinese TV series apart from the continuous drama series popular in other societies. ${ }^{46}$ Actual 'soap operas' that are continuously produced over years are not produced in China, and neither are series that stretch over several seasons, featuring a continuously developing storyline and recurring characters throughout the individual instalments. ${ }^{47}$

In general, the entire production process of an average twenty-episode TV drama, from the development of the story idea to the distribution of the finished product, will require one year $(14,59: 33$ - 59:59). This process is supervised by a production team, which includes various producers with different responsibilities and jurisdictions, as well as the screenwriters (I have provided an overview of positions and their functions in Figure 10, based on the interviews conducted in the summer of 2007). The production can be divided into four individual phases (start-up; pre-production; filming; and post production/distribution; $12,85: 00-89: 30)$, which shall be introduced below.

\footnotetext{
40 The reasons for this difference in TV formats are highly complex, and include institutional factors (for example government regulations and market economic incentives) as well as cultural aspects (such as viewing habits or traditions of narration). These factors are analysed in depth in Chapler 3.4 and 3.5 .

${ }^{47}$ US American sitcoms and TV drama series such as Friends, Scrubs, Sex and the City, Prison Break, 24, CSI or Heroes are very popular with young Chinese audiences, who watch these programmes either over the internet or on (mostly pirated) DVDs (114, 49:29 - 53:40). However, Chinese drama productions only rarely attempt to emulate these foreign formats (an exception is the Chinese adaptation of Sex and the City, Haoxiang haoxiang tan lianai 好想好想谈恋爱，aired on the regional TV stations Tianjin TV and Southern Television Guangdong in 2004). The reason is, according to one interviewee, that the specific viewing habits of Chinese audiences do not make the production of such adaptations lucrative for producers (17, 78:58 - 82:03). This issue is discussed fully in Chapter 3.4.
} 
Figure 10: Central Members of the Production Team and their Functions

\begin{tabular}{|c|c|c|}
\hline $\begin{array}{l}\text { Chinese } \\
\text { Title }\end{array}$ & $\begin{array}{l}\text { English } \\
\text { Translation }\end{array}$ & $\begin{array}{l}\text { Description/ } \\
\text { Function }\end{array}$ \\
\hline $\begin{array}{l}\text { 出品人 } \\
\text { (chupinren) }\end{array}$ & producer & $\begin{array}{l}\text { Usually the manager of the main production company, not involved } \\
\text { in the actual details of production; responsibilities include: } \\
\text { - representing the company's financial interests, } \\
\text { - acting as a legal agent for the project, } \\
\text { - supplying the production permit. }\end{array}$ \\
\hline $\begin{array}{l}\text { 编辑 } \\
\text { (bianji) }\end{array}$ & editor & $\begin{array}{l}\text { "Responsible producer" (zeren zhipianren 责任制片人) and direct } \\
\text { superior of the creative producer, mostly in charge of } \\
\text { administrative duties; responsibilities include: } \\
\text { - assuring that the production company's interests and } \\
\text { requirements are met by the creative producer and his or her } \\
\text { subordinates, } \\
\text { - assisting in early decisions on content and choice of actors. }\end{array}$ \\
\hline $\begin{array}{l}\text { 制片人 } \\
\text { (zhipianren) }\end{array}$ & $\begin{array}{l}\text { creative } \\
\text { producer }\end{array}$ & $\begin{array}{l}\text { The central person in creative control of the production process; } \\
\text { responsibilities include: } \\
\text { - choosing a genre, } \\
\text { - devising the story idea (xuanti 选题), } \\
\text { - } \text { - securing investments, } \\
\text { - hiring a screenwriter to produce a script, } \\
\text { - approving the script, } \\
\text { - devising the project's budget plan (yusuan 预算), } \\
\text { - } \text { hiring the director, the other members of the production crew, } \\
\text { - } \text { and the actors, } \\
\text { regulanteeing quality standards of production, }\end{array}$ \\
\hline $\begin{array}{l}\text { 制片主任 } \\
\text { (zhipian zhuren) }\end{array}$ & $\begin{array}{l}\text { executive } \\
\text { producer }\end{array}$ & $\begin{array}{l}\text { In charge of the operational level of drama production; } \\
\text { responsibilities include: } \\
\text { - managing the production schedule, } \\
\text { - assuring adherence to deadlines, } \\
\text { - enforcing the project's budget plan (yusuan 顶算). }\end{array}$ \\
\hline $\begin{array}{l}\text { 执行制片人 } \\
\text { (zhixing zhipianren) }\end{array}$ & line producer & $\begin{array}{l}\text { Representative of the creative producer on set (in case of large } \\
\text { productions or multiple projects). }\end{array}$ \\
\hline $\begin{array}{l}\text { 监制 } \\
\text { (jianzhi) }\end{array}$ & $\begin{array}{l}\text { production } \\
\text { supervisor }\end{array}$ & $\begin{array}{l}\text { Today largely a prestige title (similar to "associate producer"; } \\
\text { lianhe zhipian 联合制片) with no jurisdiction or decision-making } \\
\text { competency. Formerly a media professional and (usually) Party } \\
\text { representative in charge of production quality. Traditional } \\
\text { responsibilities include: } \\
\text { - assuring the contractual compliance of the production crew } \\
\text { - members, } \\
\text { - assuring correct adaptation of script, } \\
\text { - mediating in case of production-internal conflicts. }\end{array}$ \\
\hline $\begin{array}{l}\text { 发行人 } \\
\text { (faxingren) }\end{array}$ & distributor & $\begin{array}{l}\text { Responsibilities include: } \\
\text { - nupervising marketing. } \\
\text { In case of smaller production companies, these functions are } \\
\text { fulfilled by the creative producer. }\end{array}$ \\
\hline $\begin{array}{l}\text { 编剧 } \\
\text { (bianju) }\end{array}$ & screenwriter & $\begin{array}{l}\text { Professional writer, hired to create: } \\
\text { - the story outline (5,000 characters), } \\
\text { - the plot outline (1,000-2,000 characters per episode), } \\
\text { - the script. }\end{array}$ \\
\hline
\end{tabular}




\section{2.i Start-Up Phase}

During the earliest phase of Chinese TV drama production, the story idea is devised and the main discursive themes of the drama defined (I2, 85:00 - 89:30). The primary decision-maker during this period is the creative producer (zhipianren 制片人; $13,09: 08-10: 56$ ). He or she is in creative control of the production (I4, 28:51 - 29:14). When devising the story idea, the creative producer essentially has three main options (14, 08:44 - 12:57):

1. The adaptation of a literary theme to the screen. 48

2. The development of an original story idea, usually with a certain target audience in mind (in many cases, the producer will not create the idea himself, but will hire a professional screenwriter to present an original concept, often only providing guidelines regarding the genre of the drama).

3. A production based on an upcoming festivity, such as a national commemoration day, anniversary or international event. ${ }^{49}$

Once the story idea has been devised, the creative producer will hire a professional screenwriter (bianju 编剧; in some cases a team of screenwriters will be hired) to produce a story outline (gushi ganggai 故事纲概 or gushi dagang 故事大纲). The time required to create a story outline can be as short as half a month (I8, 30:12 - 31:56). This synopsis is usually about 5,000 characters long $(13,03: 06-04: 45)$ and is used to attract investors. After the outline has been written, the screenwriter(s) will summarise each episode in a plot outline, which can include between 1,000 and 2,000 characters per episode $(13,03: 06-04: 45 ; 18,4: 59-5: 51)$. The time required to create a plot outline can be as short as half a month (I8, 30:12 - 31:56). Once

\footnotetext{
48 This can include both topics of classical literature (gudai wenxue 古代义 $¥$ ) or the adaptation of more contemporary novels. An example from classical literature is the famous Dream of the Red Chamber, Hong lou meng 红楼梦 (111, 50:00 53:54): Currently, Hu Mei 胡玫, the director of HWDD, is producing a new TV drama adaptation of this Chinese classic (jingdien 经典); the series is scheduled to be aired before the Beijing Olympic Games in 2008 (the production details to this program can be found online on http://news.xinhuanet.com/photo/2006-08/22/content_4991242.htm [25.01.08]).

${ }^{48}$ For example, on the occasion of the ten-year anniversary of Hong Kong's retum to Chinese rule, the drama "Hong Kong Sisters" (Xianggang jiemei 番港姐妹) was aired in June 2007 (14; 05:19 - 08:43). Various productions are planned that directly or indirectly focus on the 2008 Olympic Games in Beijing, and the $60^{\text {th }}$ anniversary of the foundation of the PRC is already targeted by CCTV as a major event for which $\mathrm{N}$ dramas need to be produced (16; 57:04-58:00). Themes centred on such events not only offer an opportunity to create didactical or propagandistic content, they also significantly increase the possibilities of marketing a drama $(14,08: 44-12: 57 ; 17$. 15:44 - 17:24). The potential commercial success of such dramas therefore makes them attractive projects, both for state-owned and non-governmental production companies (ibid.).
} 
this work step has been concluded and reviewed by the creative producer, the screenwriter creates the actual script (juben 剧本). ${ }^{50}$ In this sense, the screenwriter develops the discourse strands of the TV drama, but only within the framework that the creative producer has provided. ${ }^{51}$

When the screenwriter has finished the script, the creative producer and (in the case of large production units) the editor (bianji 编辑) will check if the finished text satisfies the company requirements (12, 28:32 - 29:29). If no revisions need to be made, the production company will buy the script. This will usually include the rights to the drama's title and all of the characters. 52 The script is rarely altered once it has been approved, and certainly not after filming has commenced, since that would be viewed as a serious disruption of production (I1, 08:46 - 09:44; I2, 29:30 - 30:08).53 Writing the script usually takes two months (I8, 30:12 $31: 56) .54$

${ }^{50}$ One interviewee criticized this process of first writing a plot outline and then later developing the script as excessively restrictive (18, 6:58 - 8:03). According to the interviewee, this procedure is a new development in China: former generations of screenwriters would write the entire script chronologically, without a plot outline. Since the production companies had problems controlling that type of creative process, they introduced requirements such as the story outline.

54 The actual creative process of writing the script for a TV drama is usually entirely in the hands of the screenwriter(s). There are, however, cases in which other members of the production crew influence the screenwriter's work. One interviewee complained that he had been asked on numerous occasions to include new characters or storylines into his scripts $(112,68: 24-72: 20)$. He stated that such requests were particularly problematic if they came from persons who had no experience with screenwriting. such as investors from outside of the media sector. According to the interviewee, the problem is that requests from investors rarely reflect actual financial considerations (for example the inclusion of product placements); such considerations are either taken into account before the screenwriting process begins, or can be edited into the story once filming has started. More commonly, requests reflect personal preferences. This may include ideas on how the story should evolve or how characters should be developed. Since such requirements do not consider the overall storyline, such demands can seriously damage a TV drama (ibid.). They also make it difficult to pinpoint who, aside from the creative producer and the screenwriter(s), has influenced the discourse presented in a script.

52 There are cases in which the screenwriter retains the rights to the programme's name and is paid by production companies if they wish to produce sequels, spin-offs, etc; however, this is very rarely the case in China (112, 22:30 - 24:09). More commonly, the screenwriter sells the rights to the story idea along with the script. The production company can then go on to produce whatever additional content it wishes to create. An example is the TV drama NCNM: the production company Beijing Xingguang International (北京里炕国际传媒) bought the script and the copy-right to the series' concept for roughly 300,000 RMB. It has since produced a movie by the same title, a follow-up drama entitled Nucai Nanmao 女才男独, and a sequel to the originai drama (18, 17:06 - 18:23). ${ }^{53}$ There are rare cases in which the script is altered after it has been approved. A good example for such a case is the production of the drama NCNM. According to two inside source $(18,9: 19-11: 09 ; 112,14: 40-16: 54)$, the screenwriters had to heavily rewrite the script once production had started: the production company was able to hire the popular actor Lu Yi 陆数 to play one of the male roles, hoping that this choice would increase the attractiveness of the drama and facilitate promotion. However, since the original drama was devised as a story about three girls, the script only featured male support roles. When the producers realized that the actor Lu Yi would have only very little screen time (about ten scenes per episode; main characters usually have around thirtyfive to forty scenes per episode) while receiving a relatively high salary (roughly 150,000 RMB per episode, 


\section{2.il Pro-Production}

The purchase of the script concludes the creative process of the start-up phase and initiates the pre-production phase, which usually requires two to three months $(12,85: 00-89: 30)$.

During this phase, the creative producer hires the production crew and cast. The first person to be hired is the director (daoyan 导演). This brings an additional creative force to the preproduction process: The director offers advice when it comes to hiring the rest of the project's personnel (including the actors), and very often brings a pre-selected production crew to the project (for example cameramen or gaffers that the director has successfully worked with on past projects; I4, 50:25 - 53:50)..55 In addition, the director is involved in the preparations of the actual production (I1, 12:22 - 14:20), for example scouting locations (xuanjing 选景; usually with the aid of the art director; meishu 美术; 14, 46:07 - 47:29) and overseeing work on the storyboard (fenjingtou juben 分镜头剧本) ${ }^{56}$, thereby effectively defining what visual mode the various discourse fragments will later receive during the filming process.

\section{2.iil Filming}

The actual filming of the drama is usually a very fast-paced process: up to 140 scenes are shot each day $(13,21: 01-23: 43)$. In total, an average production requires approximately three to four days for the filming of one episode (14,53:51 - 56:16). This means that the filming of a twenty episode TV drama will usually be concluded within sixty to seventy days, and will

amounting to a total 1,200,000 RMB for the entire series), they had the script rewritten to include an actual male protagonist (the character of Qiu Shi 邱石). In order to accomplish this, the screen time of another character (Chen Xiaofang 沉小芳; played by the Shanghal actress Wu Jiani 只住尼) was extensively cut. Consequently, the commercial interests of the production company turned the drama into a story about two girls instead of three. and introduced discourse strands (for example on entrepreneurial success and technology) that would have otherwise not been included in the drama.

54 There are productions that require much more time for screenwriting. For example, it look the screenwriters of NCNM between five and six months to finish the script, including all revisions that had to be made (112, 60:1862:29). The screenwriters of GAJZ worked on the script for the police drama for almost four years (113, 71:08 72:31). However, these are exceptions: normally, the script to a drama will be written in a relatively short period of time $(18,30: 12$ - 31:56).

${ }^{55}$ In China, production crews for normal-sized projects on average encompass approximately 100 persons (I1. $11: 38$ - 12:21).

${ }^{50}$ For a Chinese instruction manual on how to create storyboards and organize pre-production, see Chen et al. (2005, 322-329). 
only very rarely exceed eighty days (I1, 14:21 - 14:49; I2, 85:00 - 89:30).57 During this entire period, the director takes creative control of the project and shapes the way in which the discourse fragments of the script are presented on screen. The director is responsible for arranging and shooting the individual scenes, managing the cast, and coordinate the interests of different production crew members (I1, 24:18 - 25:16), such as cameramen (sheyingshi 摄影师)，gaffers (dengguangshi 灯光师), makeup-artists (huazhuangshi 化妆师)， sound engineers (luyinshi 录音师), etc. If the project is a larger one, or if multiple projects need to be tackled at the same time, one person may function as the 'main director' (zong daoyan 总导演), supported by a number of 'assistant directors' (fu daoyan 副导演; I1, 14:50 16:38). In such cases, the main director is in creative charge of the filming process, has to assure general continuity, and generates the appropriate atmosphere for the TV drama (I2, 66:31 - 72:59). The assistant directors, on the other hand, oversee more practical details of production. This may include directors who are responsible specifically for the actors' schedules (so called 'acting directors'; yanyuan daoyan 演员导演), managing at what time what actors will work on which set (I1, 14:50 - 16:38). It may also include so-called 'on-site directors' (zuo xianchang daoyan 做现场导演), who implement the main director's instructions on the set, i.e. instructing the production crew members and the actors (ibid.). Based on the interview statements discussed here, I have presented an overview of the director's team and the function of each member in Figure 11.

\footnotetext{
${ }^{57}$ More elaborate productions may require five to six days of filming per episode (100-120 days for a twenty episode drama 14,53:51 - 56:16), but there are also cases of low-quality productions that will film one episode in two to two-and-a-half days (forty to fifty days of filming; 12, 85:00-89:30).
} 
Figure 11: Directors

\begin{tabular}{|c|c|c|}
\hline $\begin{array}{l}\text { Chinese } \\
\text { Title }\end{array}$ & $\begin{array}{l}\text { English } \\
\text { Translation }\end{array}$ & $\begin{array}{l}\text { Description/ } \\
\text { Function }\end{array}$ \\
\hline $\begin{array}{l}\text { 导演 } \\
\text { (daoyan) }\end{array}$ & director & $\begin{array}{l}\text { In creative charge of filming the drama; responsibilities } \\
\text { include: } \\
\text { - advising the creative producer with regards to personnel } \\
\text { decisions, } \\
\text { - creating the story board, } \\
\text { - scouting locations, } \\
\text { - overseeing the filming process, } \\
\text { - copervising the editing work, } \\
\text { members and actors on set. }\end{array}$ \\
\hline $\begin{array}{l}\text { 总导演 } \\
\text { (zong daoyan) }\end{array}$ & main director & $\begin{array}{l}\text { In case of large productions, the main director will supervise } \\
\text { filming at the macro-level, while various assistant directors } \\
\text { (fu daoyan 副导演) will represent him or her on set. } \\
\text { Responsibilities of the main director include: } \\
\text { - supervising the filming process on multiple locations, } \\
\text { - assuring general continuity, }\end{array}$ \\
\hline $\begin{array}{l}\text { 演员导演 } \\
\text { (yanyuan daoyan) }\end{array}$ & $\begin{array}{l}\text { acting } \\
\text { directors }\end{array}$ & $\begin{array}{l}\text { A type of assistant director, responsible for coordinating the } \\
\text { schedules of the respective actors. }\end{array}$ \\
\hline $\begin{array}{l}\text { 做现场导演 } \\
\text { (zuo xianchang } \\
\text { daoyan) }\end{array}$ & $\begin{array}{l}\text { on-site } \\
\text { directors }\end{array}$ & $\begin{array}{l}\text { A type of assistant director, representing the main director } \\
\text { on the individual sets; responsibilities include: } \\
\text { - Managing on-set logistics, } \\
\text { - Coordinating production crew members on the set, } \\
\text { - Directing actors' performances. }\end{array}$ \\
\hline $\begin{array}{l}\text { 美术 } \\
(\text { meishu })\end{array}$ & art director & $\begin{array}{l}\text { Supervises all aspects of a production's art design and } \\
\text { advises the director with regard to location scouting and } \\
\text { other artistic issues. }\end{array}$ \\
\hline
\end{tabular}

In general, the jurisdiction over the filming process will lie with the (main) director, or as one interviewee put it: "the director has the last word" (daoyan shuo suan 导演说算; I2, 11:28 -

13:48). Simultaneously, the jurisdiction for the project in its entirety will lie with the creative producer (“the creative producer has the last word”; zhipianren shuo suan 制片人说算; I3,

09:08 - 10:56; I4, 28:51 - 29:14).

\section{2.iv Post-Production}

When all material has been shot, the post-production phase will commence. During this time, the filmed material is edited (jianji 剪辑 or jianjie 剪接) and possibly (though only very rarely) 
re-shot (I1, 14:21 - 14:49; I4, 59:33 - 59:59). This period requires between three and five months of work (ibid.).

All throughout the filming and post-production phases, the creative producer will already market the TV drama for distribution (I4, 56:17 - 59:32). He or she is also in charge of negotiating the sale of the drama. In this sense, each phase of production can be handled flexibly, and in most cases the different phases will to some extend overlap, particularly when it comes to marketing aspects (ibid.). In general, the entire production process of an average twenty-episode TV drama, from the development of the story idea to the distribution of the finished product, will require one year (14, 59:33 - 59:59).

\subsection{Selling the Drama}

According to the interviewees $(12,18: 05-20: 42 ; 14,56: 17$ - 59:32 \& 76:42 - 80:25; I12, 44:11 45:20), as the creative producer attempts to market his product, he or she will take into account various potential sales models, as well as the requirements from possible buyers (i.e. what type of television dramas the TV stations will regard as a 'good' drama). However, even a production unit that creates TV dramas that fulfil the needs of buyers will still rely on a skilful distribution unit with an appropriate marketing strategy: As one interviewee put it, "the scent of alcohol is lost in a small alley" (jiuxiang pa xiangzi shen 酒香怕巷子深), meaning that a good product will not be sold if it is marketed badly $(13,27: 48-30: 50)$. This is why marketing issues already play a role during the earliest stages of production, for example when it comes to hiring production crew members and casting actors. For this reason, it is necessary to consider how sales considerations influence the production of TV drama content.

\subsection{Distribution: How TV Series Are Marketed}

Before turning to the various requirements posed by the Chinese TV drama buyers, it is worth taking a look at the actual prices that are paid for TV dramas, the sales models that 
production companies use to distribute them, and the market dynamics that influence drama sales.

As one interviewee explained, Chinese TV dramas are sometimes sold to broadcasting stations overseas $(110,19: 30$ - 20:00), but the impact of these programmes on foreign markets is not particularly high. The target audience for Chinese drama exports are mostly overseas Chinese, and for this reason the markets in South-East Asian, particularly Singapore, are most relevant (I12, 50:51 - 52:22). Some dramas are also sold to TV stations in Korea or Japan (for example, the family drama Chinese-style Divorce, Zhongguo shi lihun 中国式离婚， was sold to the Japanese station $\mathrm{NHK}$ ), but the domestic market remains the predominant target for Chinese TV drama distribution (I2, 03:36 - 05:30).

The sales process in mainland China is governed largely by market economic dynamics ( 12 , 76:16 - 78:17). There are still production units that will profit from their affiliation with state television stations, but the majority of production companies will have to sell their product on the open market (ibid.). Broadcasting stations in the prosperous urban regions of eastern China (for example in the cities of Beijing or Shanghai, as well as in provinces such as Jiangsu or Zhejiang) may in some cases pay up to ten million RMB for the rights to air an entire series (I2, 18:05 - 20:42), but for most stations in developed regions the rights to broadcast a twenty-episode TV drama will be somewhere in the vicinity of 1.5 million RMB (I1, 20:48 21:42). Even if a drama is sold to a TV station at a relatively high price, the returns from that one sale will rarely cover the production costs; production companies therefore need to sell their products to numerous TV stations, and will usually try to find at least ten different buyers (12, 18:05 - 20:42).

Considering the size and different developmental levels of the Chinese market, TV drama prices vary widely, depending on whom they are sold to (12,25:44 - 27:34): TV stations in more developed parts of China (such as the Dongbei region, Shandong Province, Jiangsu 
Province, Zhejiang Province, Beijing, Shanghai, etc.) pay approximately 50,000 RMB per episode (ibid.). A special case is the highly developed province of Guangdong; due to the strong influence that Hong Kong TV stations play in that region, broadcasters in Guangdong pay far less for an episode of TV drama than their colleagues in other prosperous regions, usually only 10,000 RMB per episode (ibid.). Finally, broadcasting stations in underdeveloped regions (Xinjiang, Tibet, Qinghai, etc.) pay very low prices, sometimes only a couple of hundred RMB per episode (ibid.). Such sales consequently do not play a large role; production companies usually make about eighty to ninety per cent of their profits from sales to the developed regions (I2, 18:05 - 20:42). This means that TV dramas will mostly be created with these markets in mind.

According to one interviewee (I4, 76:42 - 80:25), there are generally two sales models for TV dramas: A programme can be sold at a predetermined fixed price to one (or more) television stations (for example, a broadcaster like CCTV, or a regional television station such as BTV, will pay 50,000 RMB per episode for the right to air a TV drama; ibid.). A more common method is to sell the programme to TV stations at a price that depends on later TV ratings (for example five per cent prime-time ratings entitles the distributor to the normal 50,000 RMB per episode; lower ratings decrease the profits, while higher ratings increase them; ibid.). In some cases, the distributor may contractually agree to sell the broadcasting rights exclusively to one primary buyer until the drama has successfully been aired: This may be the case with large TV stations (such as CCTV) who want to assure that the drama's ratings are maximised (112, 44:11 - 45:20).

If a TV drama is received favourably by audiences, a DVD or VCD release shortly after the first broadcasting may create additional revenues (16, 43:06 - 45:29). However, such a release is not handled by the respective production units themselves, but instead by independent distributors $(12,103: 40$ - 107:22). Such distributors buy the rights to DVD sales at a fixed price 
(ibid.). Due to DVD piracy and illegal internet downloads, the sales from this market segment have continuously decreased over the past years $(15,46: 06-47: 16)$. DVD editions of TV dramas used to have a nation-wide circulation of about 20,000 copies; today, production has dropped to about 2,000-3,000 copies per series (I2, 103:40 - 107:22). A legal copy of a full drama series on DVD is usually sold at a price ranging from $80 \mathrm{RMB}$ to about $300 \mathrm{RMB}$, depending on the length and popularity of the programme. At a circulation of 3,000 copies, this would bring the revenues from DVD sales to somewhere between 240,000 RMB and $900,000 \mathrm{RMB}$ per series. Another very small distribution opportunity is to offer series for legal internet download. Such offers are also handled by independent distributors, who pay a small fee (roughly $500 \mathrm{RMB}$ per episode; $12,103: 40$ - 107:22) for the right to offer the respective service.

How the total profits from TV drama sales are divided between the various investors depends on the contractual model that was adopted at the start of the production process. According to the interviewees, there are two such models: The investors can either choose to receive a pre-defined, fixed amount of money once the product has been distributed (guding huibao 固定汇报，for example $\times \%$ of their original investment; $15,39: 43$ - 41:22). Such an approach will minimise the investors' risk (I4, 25:52 - 27:23). Alternatively, more risk-seeking investors may choose to receive a share of the final profits. This share will usually reflect the investor's financial involvement in the project (investing $\mathrm{y} \%$ of a project's total budget will consequently entitle an investor to $y \%$ of the final profits; $I 2,14: 08-15: 39$ ). As one interviewee stated, the total volume of revenues generated by TV drama sales in China amounts to roughly three billion RMB each year (I14, 27:20 - 31:14). The series NCNM is a good example of the revenues that a successful twenty-episode TV drama creates: the sales revenue for that particular programme totalled twelve million $\mathrm{RMB}$; with a production cost 
of seven million $\mathrm{RMB}$, leaving the production company and the investors with five million RMB in profits $(112,50: 51$ - 52:22).

\subsection{The Buyers: TV Stations and their Motives}

A main characteristic of the TV drama market is a noticeable over-supply: Roughly 10,000 episodes $^{58}$ of television drama are produced in China each year (I6, 28:33 - 30:40), which amounts to a total of 700 to 800 series, of which only about thirty per cent are actually aired (12, 45:39 - 46:56). The massive over-production substantially increases competition on the side of the production companies $(15,71: 26-80: 00)$. This makes the Chinese TV drama market a buyers' rather than a sellers' market $(19,43: 38-45: 42)$. It puts the TV stations in a very powerful bargaining position, enabling them, as one interviewee put it, to act as the "overlords" (bawang 霜王; [3, 35:21 - 37:12) of the market: large TV stations in particular (for example CCTV) use the attractiveness of their prime time broadcasting slots (huangin shijian 黄金时间; 8-10 p.m.) as pressure against production companies, dictating the terms of purchase ("TV stations have the last word", dianshitai shuo suan 电视台说算; ibid.) and driving the prices of TV dramas down. One effect is that TV drama quality is reduced (13, 45:16 - 47:06); another that production companies censor themselves by avoiding controversial topics and tailoring the content of their productions to the requirements of the individual TV stations $(13,35: 21$ - 37:12).

This means that production companies have to decide early-on during the production process to what type of TV station they ultimately wish to distribute their product (I14, 33:00 - 34:11): The preferred sales option will be to sell a series to CCTV; this will generate the maximum amount of profits and decrease the financial risks for the producers (114, 27:20 31:14). However, it may at first limit other distribution options (CCTV will usually request the exclusive rights to air the respective dramas; $15,43: 21$ - 46:05). As a second option, TV 
dramas will be sold to regional TV stations; however, the profits from such sales will be far less than if the product were sold to CCTV $(12,18: 05$ - 20:42). A final option is to sell TV dramas to satellite stations (weixing dianshitai 卫星电视台), but most production companies will only consider this market after all other distribution options have been exhausted (114, 33:00 - 34:11): selling programmes to satellite stations eliminates any further chances of marketing the products, since it technically enables audiences all around the world to view the respective dramas.

Considering the diversity of the Chinese broadcasting sector, the greatest challenge for production companies is, according to one interviewee, to create a drama that will satisfy the demands of as many TV stations as possible (110, 58:18 - 59:28). Regional TV stations will emphasise entertainment (yule 娱乐), whereas CCTV (particularly CCTV-1) will require programmes with more serious (or 'healthy'; jiankang 健康) content and a distinct focus on education and ideology, preferably so-called 'main melody' dramas (110, 21:30 - 22:29).

The criteria that both production companies and TV stations adopt in order to define what constitutes a 'good' or 'healthy' TV drama are summarised by the slogan "unite the three qualities" (sanxing tongyi 三性统一). These are (12, 52:47 - 53:35; 16, 03:05 - 05:37; 17, 11:26 12:26; I10, 04:31 - 07:54; I11, 27:23 - 29:18; I13a, 56:58 - 59:44):

1. Ideological quality (sixiangxing 思想性),

2. Artistic quality (yishuxing 艺术性), and

3. Viewing quality (guanshangxing 观赏性).

These phrases remain sufficiently vague, and most interviewees were hard pressed to formulate distinct definitions for each of these 'qualities' (ibid.): Generally, ideological quality means that content is in line with the respective state guidelines, artistic quality seems to refer to camerawork, lighting, sound, acting, set and costume design, etc., and 
viewing quality is equated with audience appeal, i.e. is measured in terms of TV ratings. I will return to the matter of ideological quality in chapter 3.5 , and to the other criteria in chapter 3.7.

\subsection{The Chinese Television Drama Audience}

In order to successfully sell a TV drama, production companies need to take into consideration not only the various buyers' preferences, but also the potential target audience of their product. The interviews generally confirmed what other authors have already argued (see section 2.2.iii), i.e. that TV stations increasingly rely on advertisement profits: as one interviewee stated, small stations in particular sometimes receive sixty to seventy per cent of their revenues from airing commercials during breaks (I4, 80:26 - 84:48). This makes the question of what audiences watch at what time a major concern to the entire industry.

\subsection{Assessing Audiences: the Chinese Audience Measurement System}

As described earlier (chapter 2.2.iv), audience research in China is a relatively recent enterprise, and is to-date still highly imperfect. The dilemma is that TV ratings (shoushilü 收 视率) do not reflect the actual reactions of audiences, yet are nonetheless the main instrument with which advertisers, TV stations and production companies assess the marketing value of drama series (I2, 31:31 - 33:24): the TV stations rely on the ratings in order to sell advertisement slots, and the production companies require ratings to sell (and possible later resell) their products to the broadcasters. ${ }^{59}$ One producer voiced his frustration with the current system by stating that "the ratings completely dominate this programme market of ours, they completely dominate over me!" (shoushilu wanquan zhuzai women jiemu de zlie ge shichang, wanquan zhuzai wo! 收视率完全主辛我们节目的这个市场, 完全主幸我！ I2, 126:51－ 128:15).

\footnotetext{
${ }^{59}$ For producers, the ratings of the first broadcasting run are consequently the most important: they determine how much the respective TV station will eventually pay for the drama and how likely it is that a TV series will later be bought by other stations $(112,44: 11-45: 20)$.
} 
Despite these objections, the demand for data on TV drama audiences is nonetheless so strong that turning to questionable statistics is still preferred over not having any data at all $(114,36: 54-41: 10)$. The implications of this situation are considerable: the relevance of the available audience measurement data provides producers with incentives to create TV dramas entirely aimed at the viewers that are included in the statistical sample, instead of producing formats that would appeal to broader (but statistically unaccounted for) audiences (17, 82:04 - 84:15).

In this context, producers are presented with a series of major problems that arise from the way in which audience ratings are created in China:

1. The two largest providers of audience ratings, i.e. CSM and AGB Nielsen (see Section 2.2.iv), calculate different types of audience ratings (15, 47:13 - 51:09): for example, CSM will provide the highest ratings that any one episode of a specific TV drama series received, whereas AGB Nielsen will offer an average value (pingyue shoushilu 平均收视率) for the entire series.

2. The fact that the CSM and AGB Nielsen base their calculations on different samples (yangben 样本) ultimately creates different ratings' results (14, 80:26 - 84:48; 16, 30:41 - 33:21).

3. Numerous interviewees have criticised that CSM and AGB Nielsen both used insufficiently large sample bases (I2, 31:31 - 33:24; I4, 80:26 - 84:48; $15,47: 13-51: 09 ; 17,82: 04-84: 15$ ) ${ }^{60}$ : whether 11,000 or 54,000 households, the data derived from this sample was regarded as hardly representative for the $\mathbf{1 . 2}$ billion Chinese citizens who potentially watch TV dramas. Instead, the sample base is said to represent mostly the urban, middleaged, female viewers in China (16, 30:41 - 33:21).

4. The system according to which the ratings are calculated is not transparent $(13,109: 39-115: 33)$.

5. The ratings are presented in tables that are structured along excessively large units of time, so that the data often only reflects highest values achieved throughout an entire year (ibid.).

6. The relevant data is not commonly available to the public (ibid.).

\footnotetext{
00 The problems described here merely relate the fact that the current measurement system does not accomplish what it promises, i.e. assessing what TV programmes are most popular. Some of the interviewees further questioned whether the most popular series are in fact also the qualitatively best series (12, 35:48 - 38:00; 19 , $09: 20$ - 09:43). As valid as this objection may be, it shall not be debated here.
} 
7. The quantitative data is not augmented by qualitative analytical criteria (I14, 36:54 - 41:10), so that the resulting data offers no information as to why audiences decided to tune in for a specific programme $(15,47: 13-51: 09)$.

8. In some cases, extremely high but unlikely ratings results suggest that the data were altered intentionally, and that the system may be subject to corruption (15, 47:13 - 51:09). One interviewee, who did not want her comment recorded, pointed out that the proximity of CSM to the CCTV wholly-owned subsidiary CITVC invites misuse: according to the interviewee, China's central television station is essentially analysing itself and its competitors, and this does not produce reliable data.

Researchers and analysts of the Chinese TV drama market are aware of these problems ( 13. 109:39 - 115:33), which may be why Chinese academics predominantly approach the subject of TV drama from the content side and choose to ignore the perspective of audiences (ibid.).

Broadcasters and production companies are equally aware of the flaws in the audience measurement system and have in some cases started to conduct their own research of viewing preferences in order to assess which TV dramas are likely to be successful and should therefore be aired at times were advertisement revenues are highest (for example, Nanjing TV frequently test-screens TV dramas to a selected group of forty viewers in order to assess audience reactions; $12,45: 00-45: 38$ ). However, individual surveys are still very rare. If broadcasters wish to assess the potential popularity of a specific TV drama, they usually turn to the solutions offered by the leading market research companies. According to one interviewee $(17,59: 58$ - 64:24), CTR (in cooperation with CSM) offers specific research, tailored to the needs of individual broadcasters. According to the interviewee (ibid.), the method is usually to invite a group of research subjects to a hotel and provide them with individual accommodation. Each room features a television set that shows the different TV dramas that are under scrutiny. The research company then measures what type of person watches which series for how long. After the experiment, each research subject is asked to fill out a qualitative questionnaire in order to assess the reasons why the different viewers chose certain programmes over others, and what aspects (if any) they found particularly 
entertaining. The data gathered from these experiments is then used by the broadcasters to decide which TV drama they wish to buy, and how they should best promote the programmes. In general, however, these types of test-screenings are still the exception in China, and most editors, producers and television station executives are forced to rely on past experiences in order to assess the marketing value of TV dramas and their potential impact on audiences $(17,64: 25-65: 05)$.

\section{4.ll The Target Audiences of TV Dramas}

Television drama, as a form of entertainment, is generally believed to appeal to audiences of all backgrounds and all ages in China (I6, 10:13 - 11:39; $110,17: 46$ - 18:06). However, even though some television dramas like the ones analysed in Chapter 4 are able to attract a broad audience, most TV dramas are watched by a very narrow group of viewers. When asked who the typical Chinese TV drama viewer was, the interviewees largely confirmed the assessment provided by $\mathrm{Li}$ Jingcheng and his colleagues from the CUC ( $\mathrm{Li}, 2007$; see section 2.2.ii). The conclusion from my research can be summed up as follows $(12,38: 01$ - 39:28; I6, 10:13 - 11:39; [11, 33:24 - 34:40; I14, 46:49 - 47:43):

1. Drama viewers are believed to be predominantly female and generally older than 45. It is possible that this is due to the television content on offer, and that the majority of Chinese television dramas appeal primarily to older viewers than to the younger generation. However, as various interviewees have pointed out, the main reason for this age discrepancy is a general difference in life-style between the young and the old generation: Young urban viewers are usually occupied with their careers and have neither the time nor the interest to watch television dramas $(13,115: 34$ - 121:30). What little leisure time this age group has is usually spent away from home: in KTV bars or clubs, watching films at movie theatres, or playing online computer games in internet cafés (I4, 84:49 - 88:32). When these younger viewers do watch Chinese television dramas, they tend to download the programmes from the internet and watch them on a computer rather than tuning in for the actual television broadcast $(111,33: 24-34: 40)$. Whatever the reasons for this difference in viewing habits, the result is that the television drama broadcasts do not aim at viewers under the age of 45 , but instead at, as one interviewee put it, the "mama generation" (18, 62:01 - 64:11), i.e. married older female housewives who spend their evenings (or generally their days) at home $(14,84: 49$ - 88:32). 
2. The major groups targeted by Chinese television dramas are believed to be viewers with low-wage jobs, laid-off workers, retired viewers, and migrant workers $(13,115: 34-121: 30)$, i.e. viewers with low incomes and low levels of education (16, 33:22 - 35:15).

3. According to one interviewee, northern viewers generally tend to watch more television dramas than southern viewers; the reason for this is supposed to lie in the climatic differences between the regions (I14, $46: 49$ - 47:43).

What is relevant in this context is that there is believed to be a general discrepancy between the audience that is measured by the ratings system (and hence targeted by advertisers and broadcasters) and the viewers who actually watch drama series, particularly CCTV dramas: As one expert stated, the actual group that is most likely to watch drama series is predominantly rural $(13,115: 34-121: 30)$. This means that the actual (rural) audience of TV dramas is not the same (urban) audience for whose consumption the dramas are produced (ibid.).

\section{4.iil Vlewing Habits: How Chinese Television Dramas Are Watched}

The above assessment has shown that TV drama series are produced primarily for an urban, female, middle-aged audience with low-levels of income and education. This begs the question of whether any particular viewing preferences of this particular target audience influence the way TV series are devised and aired. In order to answer this question, it is worth taking a short detour from the factual statements that the interviewees made regarding drama production and broadcasting, and to examine in more detail what perceptions and expectations they held towards audiences. My research shows that media experts in China believe the Chinese audience to have very different viewing habits from foreign audiences, and that these supposed difference are used to explain and justify the peculiarities of drama serials that were described in section 2.2.ii (12, 129:33 - 130:12; 12 , $136: 40$ - 137:39; I6, 17:34 - 19:20; I7, 78:58 - 82:03; I14, 49:29 - 53:40). In these lines of argumentation, two tropes are repeatedly used: 
The first trope is that Chinese viewers are less patient than Western audiences, and that they demand an immediate continuation of the series they are watching. One interviewee summed this up as follows $(12,128: 14-129: 32)$ :

I2: As for Chinese television dramas, once you've started broadcasting you have to continuously broadcast. You broadcast everything quickly. The viewers want to watch continuously, they won't wait for you.

The second trope is that most Western television series are 'slower' than Chinese programmes, and that Chinese viewers (supposedly in contrast to Western viewers) prefer fast-paced action and no loose ends. The following interview segment makes use of both these tropes (114, 47:44 - 49:19):

R: So why is it that Chinese television dramas are completely different from those in the West? Like you just said: every evening you have two episodes, and every day it's the same television drama, and then after one or two weeks the broadcasting has been concluded, right? This is completely different from the Western way of showing one episode per week. Why does China broadcast like this?

114: There are reasons for this in China. The first reason is the human nature of the Chinese people. That is, Chinese people are much more impatient than Westerners. Chinese people want a very fast conclusion. They don't like a long period of suspense. The most suspenseful dramas will still sell in China... extremely suspenseful ones will sell. But Chinese people can't stand long waits. They'll stop watching. (...) For example Prison Break: they will prefer to watch that, because every episode solves a particular problem comprehensively. Lost on the other hand they won't watch, because they feel it is too slow. They have to wait for that conclusion. They have to wait too long to understand the final conclusion. So if you showed Prison Break and Lost at the same time, then certainly Prison Break would have the higher ratings. Most certainly. Because Prison Break has a forceful ending for every episode. Such forceful endings are very important for serials.

As the above example illustrates, the argument that Chinese audiences have particular viewing preferences is also made when it comes to the appeal of foreign television content appeal to Chinese viewers: it is argued that Western programmes are for the most part too exotic and too far removed from the experiences of Chinese audiences (I6, 49:04 - 51:59; 17, 78:58 - 82:03) and that the cultural and language barrier would prevent the average television viewer from watching such programmes $(15,61: 10-65: 39)$. This is also why Chinese media 
experts generally believe that the government's restrictions of foreign content on Chinese television are largely redundant $(16,49: 04$ - 51:59; 17, 78:58 - 82:03): Chinese broadcasters have little incentive to invest in foreign series, and even if they did broadcast Western programmes, so the argument goes, the average Chinese viewer would not be interested in watching them.

It is important to point out, however, that not all interviewees assumed that the preference for certain forms of drama presentation were a result of a specific Chinese personality trait, as is suggested in the interview excerpt above. The popularity of Western programmes with young viewers, for instance, is in some cases understood as proof of a generational difference in viewing preferences $(17,78: 58$ - 82:03). The reason, for example, why Western series sell well on DVD but not as TV broadcasts is interpreted as a result of audience demographics: if a programme such as Prison Break or Lost was aired on Chinese television, so the argument goes, the average viewer (the urban female housewife, age 45 or above) would not be attracted by the series content, and the actual target audience (i.e. young viewers with more diverse viewing preferences) would not tune in because they had already watched the series on the internet $(15,61: 10-65: 39)$.

In this sense, it is debatable whether specific supposedly Chinese viewing preferences are the main factor behind the way in which Chinese drama series are produced and aired, even though many media experts in China seem to believe so (I2, 129:33 - 130:12; I2, $136: 40$ - 137:39; $16,17: 34-19: 20 ; 17,78: 58$ - 82:03; I14, 49:29 - 53:40). ${ }^{61}$ There are two other plausible reasons why Chinese television series are conceived as closed narrations and are broadcasted en bloc:

First, the current method of producing and airing drama serials is a cheap way of providing large amounts of television content: As one interviewee explained, the production of

\footnotetext{
${ }^{61}$ It is not the purpose of this analysis 10 establish whether these preconceptions are true, or whether they are particularly Chinese; the point here is that drama production team members and broadcasters believe these preconceptions and base their production and broadcasting decisions on them.
} 
Western series requires more time than the much simpler Chinese programmes (especially when programmes are continuously produced over years) and hence involves high longterm expenses and no immediate lump-sum profit $(12,30: 49$ - 31:30). Television stations are used to filling their broadcasting schedules with cheap domestic productions; the purchase of an expensive foreign series or a domestic drama modelled on Western programmes is not regarded as a feasible investment (I6, 49:04 - 51:59).

Second, the Chinese production and broadcasting system is governed by an array of censorship regulations which is only able to function properly if drama series production is completed first, and the whole programme then submitted for censorship next; this issue is discussed below, in Section 3.6.

\subsection{Iv Drama Genres: What Audlences Watch}

As has already been mention in section 2.2.ii, various criteria exist to classify what types of television dramas audience watch. My research showed that numerous of the media experts interviewed for this project brought up genre categories of their own (15, 00:51 - 02:40; 17 , 20:53 - 26:59; I11, 05:30 - 15:35), for instance the 'political drama' (zhengzhi ju 政治剧), the 'moral love drama' (qinqing lunli ju 亲情伦理剧), the 'adaptation of classic works' (jingdian gaibian 经典改编), or the 'nostalgic family drama' (jiaxiang lunli ju 家乡伦理剧). However, the main categories that were repeatedly mentioned as the most influential drama genres were $(14,88: 33$ - 91:05 \& 93:27 - 96:47; 15, 00:51 - 02:40 \& 10:45 - 21:22; 17, 20:53 - 26:59; 18, 64:12 $68: 29 ; 111,11: 18-14: 19)$ :

1. The historical or costume drama,

2. The family drama,

3. The crime drama, and

4. The teen drama. 
Since 'main melody' themes can be associated with any of these genres, the term does not aptly signify a category of its own, but is much rather an overarching characteristic that can apply to any genre (for instance, GAJZ is a crime drama, but at the same time it promotes main melody themes; 113, 05:54 - 07:55 and 23:35 - 27:44).

When it comes to the distinction between the content of main melody dramas and purely commercial dramas, it is again useful to consider to the interviewee's personal perception; the interviewed media experts believed that there was a significant discrepancy between the two: main melody themes (i.e. content that conforms to the official criteria on ideological and artistic quality) were generally viewed as 'good' television, and most popular dramas (i.e. themes that sold well) were regarded as 'unhealthy' (I6, 45:30 - 48:33; I10, $21: 30$ - 22:29; 111 , $15: 36-16: 40 ;[13,23: 35-27: 44)$. The main concern seems to be that the most appealing themes are exactly the themes that the government views as potentially destabilizing to society (i.e. power, conspiracy and violence; $13,77: 22$ - 81:20).

Considering these arguments, it is at first sight surprising that the ratings system nonetheless registers high numbers for such main melody dramas as GAJZ or Blank Sword (Liang jian 究剑, which achieved the highest ratings among all television dramas broadcasted in 2005; Wang, $2006,233)$. One interviewee explained that particularly well-filmed main melody dramas with a good script will still draw a large audience, and will appeal to more people than merely the average middle-aged female viewer (17, 46:13 - 47:56). Another argued that famous actors and production crew members are able to increase the appeal of dramas substantially $(15,21: 23-22: 47)$. Finally, particularly historical main melody themes are believed to appeal to viewers' sense of nostalgia $(19,31: 07$ - 33:49). Although all of these arguments suggest that main melody propaganda dramas still reach a large audience, one interviewee pointed out that just because main melody series are watched does not necessarily mean they are internalised (I11, 35:09 - 37:10): CCTV main melody series are 
generally broadcasted during prime time, which is when most families eat dinner while simultaneously having the television running in the background (ibid.). This may generally mean that a programme receives high ratings, but says little about its actual viewer appeal. Most prime time television may well be watched out of habit rather than actual interest. In this sense, it is not so much the audiences who create a demand for main melody themes, but the broadcasters themselves.

\section{4.v Audience Feodback}

The reaction of Chinese audiences to television dramas has a different impact on the drama production and broadcasting system than it does in foreign markets. Chinese viewers discuss their favourite programmes on the message boards of entertainment portals such as sina.com (ent.sina.com.cn) or on the homepages of television stations such as CCTV (www.cctv.com/teleplay), but these debates can by default not influence the production of the respective drama (13, 121:31 - 128:20): Whereas discussions on Western fan sites or on message boards such as those of the International Movie Database (www.imdb.com) often accompany the production of a series, the discussions in China usually do not take place until broadcasting has started and production has long been concluded. A plausible reason for this is that the audience are only aware of the dramas that are broadcasted, but rarely of those being produced, unless the series in question is a high-profile production (I11, 22:39 27:22). The kind of production that is likely to incite debate, even in the pre-production phase, is the adaptation of literary classics or important historical themes (as an example, one of the interviewees referred to the debate in China on who should be cast to play the main characters in Hu Mei's adaptation of The Dream of the Red Chamber; ibid.).62

There are, however, two channels of feedback that according to interviewees receive attention from broadcasters. The first is the Chinese press. The comments in newspaper

\footnotetext{
${ }^{02}$ For production details on this drama series, see http://ent.sina.com.cn/f/v/newhlm/index.shtml [27.07.08]
} 
editorials and the critiques of intellectuals published in the print media are used by broadcasting officials to assess whether the programmes they air are perceived as socially and politically acceptable by the Chinese elites $(15,30: 14-35: 41)$. As is described below in the section on censorship (3.5), criticism in the papers can indeed cause television station executives to take a programme off the air, move it to a different broadcasting slot, or speed up the broadcasting in order to get rid of the problematic content without drawing too much attention to it (ibid.; 13, 81:21 - 84:36).

The second channel of communication that seems to be increasingly used by audiences is the direct viewer comment that has been described by the CUC research project on TV drama audiences (see Section 2.2.iv), i.e. feedback offered to the respective TV station's audience contact department (guanzhong lianxi chu 观众联系处) in the form of letters or phone calls, but increasingly also via email, mobile phone text message, or message board post (I6, 35:16 35:55). Before turning to the question of how relevant the media experts interviewed for the present project found such feedback, I shall offer an analysis of the types of feedback that are commonly provided by Chinese viewers. For this purpose, I have translated below the sample feedback quotes that the CUC researchers use to exemplify audience reactions to TV drama series, and have treated these comments as a primary source for analysis ( $\mathrm{Li}, 2007$, 229-231). It is difficult to say how representative these selected quotes are, and in how far they reflect viewers' actual opinions on TV dramas. However, from a discourse analytical perspective, the reproduction and presentation of these comments at the very least addresses two questions:

1. What kind of feedback the CUC researchers regarded as relevant, and

2. What form such feedback takes (i.e. what the focus is, and in what language comments are presented).

First, the distinction that the CUC researchers make between positive and negative feedback deserves attention: more than eighty per cent of the viewer comment analysis is devoted to 
appraising remarks. These remarks are then divided according to the genres they address, i.e.

"realistic television dramas", "historical television dramas", "costume dramas", and

"imported drama"; I have summarised these comments in Figure 12 (ibid., 229-231; author's

translation).

Figure 12: Positive Viewer Feedback as Presented by CUC researchers

1. Realistic Dramas (现实题材电视剧)

\begin{tabular}{|c|c|}
\hline Chinese original & English translation \\
\hline $\begin{array}{l}\text { 1. 《真情不眼》十分感人, 太好了, 希望多播这 } \\
\text { 样的好剧。 }\end{array}$ & $\begin{array}{l}\text { "The Truth Can't Be Seen" is extremely moving } \\
\text { and excellent, I hope for more such good dramas. }\end{array}$ \\
\hline $\begin{array}{l}\text { 2. 《家风》剧情严谨、正气、人情味浓, 演员、 } \\
\text { 导演、音乐都不错, 希望可以重播。 }\end{array}$ & $\begin{array}{l}\text { "Family Customs" has a well-knit plot, a healthy } \\
\text { atmosphere, and dense human interests. The } \\
\text { actors, the director, and the music are all really } \\
\text { good. I hope it will be rerun. }\end{array}$ \\
\hline $\begin{array}{l}\text { 3. 《圣水湖畔》突出了尊重农民、保护耕地等重大 } \\
\text { 主题, 具有很大的现实意义。 }\end{array}$ & $\begin{array}{l}\text { "Shengshui Hupan" stresses important issues } \\
\text { such as the respect for the peasantry and the } \\
\text { protection of cultivated land. It has great practical } \\
\text { significance. }\end{array}$ \\
\hline $\begin{array}{l}\text { 4. 《圣水湖畔》源于生活, 高于生活, 有种亲切 } \\
\text { 的生活感, 既有政治意义, 又有艺术感染力。 }\end{array}$ & $\begin{array}{l}\text { "Shenghui Hupan" is rooted in life, exceeds life, } \\
\text { and possesses an intimate sentiment of life. It has } \\
\text { both political significance and artistic influence. }\end{array}$ \\
\hline $\begin{array}{l}5 . \text { 《任长霞》是一部非常好的弘扬时代主旋律的 } \\
\text { 作品, 其真实人, 催人泪下, 极具鲜活感人的 } \\
\text { 鬿力。扮演任长霞的演员, 将党的好女儿、人民 } \\
\text { 的好干部任长霞的伟大风范, 表现淋漓尽致。 } \\
\text { 《任长霞》再次感动了中国, 愿类似的作品更多 } \\
\text { 出现在中央电视台的屏幕上。 }\end{array}$ & $\begin{array}{l}\text { "Ren Changxia" is an outstanding work that } \\
\text { promotes the main melody of our age. It is truly } \\
\text { moving and tear-wrenching, and possesses a } \\
\text { lively charm that moves people. The actress that } \\
\text { plays Ren Changxia vividly displays Ren } \\
\text { Changxia's great demeanour as a good daughter } \\
\text { of the party and a good cadre of the people. "Ren } \\
\text { Changxia" moves China once more. It would be } \\
\text { great to see more such programmes on CCTV. }\end{array}$ \\
\hline $\begin{array}{l}\text { 6. 《阳光雨季》是少有而优秀的教育电视剧, 较 } \\
\text { 全面反映了以学校为主的家庭教育、社会教育综 } \\
\text { 合改革成就现状和问题. 特别是老中青教师、干 } \\
\text { 部以及各类学生的人物塑造、情节、矛盾及其转 } \\
\text { 化的描述, 真实生动, 具有科学性、现实性和可 } \\
\text { 信性, 对于当前教育教学改革有很好的启发性和 } \\
\text { 借鉴意义。 }\end{array}$ & $\begin{array}{l}\text { "A Sunny Rain Season" is a rare and excellent } \\
\text { educational television drama, it reflects rather } \\
\text { comprehensively the recent achievements and } \\
\text { problems of the supremacy of the school as a } \\
\text { means of domestic and social education, and in } \\
\text { particular depicts the characters, plot, } \\
\text { contradictions, and transformations of old, middle- } \\
\text { aged, and young teachers, cadres, and all sorts of } \\
\text { students. It is scientific, realistic, and credible, and } \\
\text { possesses significance for the present reform of } \\
\text { education and teaching which is both enlightening } \\
\text { and something we can draw lessons from. }\end{array}$ \\
\hline
\end{tabular}

2. Historical Dramas (历史题材电视剧)

\section{Chinese original English translation}

7. 《亮剑》情节跌宕起伏, 人物性格鲜明感人, 李幼斌表演真实、生动、鲜活。

8. 《冼星海》和《八路军》都是主旋律, 感人至 深, 在改革开放的今天, 重温革命战争时期革命 前辈们经历的千辛万苦, 为党、为祖国、为解放 全中国献出自己的一切, 确实打动了亿万观众的 心, 具有极强的教育意义。

The plot of "Blank Sword" is undulated and flows without decorum. The temperament of the characters is distinctly moving. Li Youbin's acting is authentic, vivid, and fresh.

"Xian Xinghai" and "The Eighth Route Army" are both main melodies and deeply moving. To in our day and age revive the untold hardship that our revolutionary predecessors had to endure during the revolutionary war, to show how they sacrificed everything for the party, the homeland, and the liberation of China, has truly moved the hearts of millions upon millions of viewers. This has an extremely strong educational significance. 


\begin{tabular}{|c|c|}
\hline $\begin{array}{l}\text { 9. 《八路军》回忆起上个世纪三四十年代的苦难 } \\
\text { 日子, 王伍福的表演表现出朱德的和䔽㥿厚, 把 } \\
\text { 朱德的军事家魕力和英豪气质展现得淋漓尽致。 }\end{array}$ & $\begin{array}{l}\text { "The Eighth Route Army" recalls the days of } \\
\text { suffering of the } 1930 \text { s and } 40 \mathrm{~s} \text {. Wang Wufu's } \\
\text { acting depicts the kindness and honesty of Zhu } \\
\text { De, and vividly unfolds Zhu De's military glamour } \\
\text { and heroic disposition before our eyes. }\end{array}$ \\
\hline $\begin{array}{l}\text { 10. 《记忆的证明》给了中国人回头看历史的真 } \\
\text { 实, 也给了看过此剧的青年人一个真实的史实。 }\end{array}$ & $\begin{array}{l}\text { "Proof of Memory" offered the Chinese people a } \\
\text { realistic view of history and also gave the young } \\
\text { viewers who watched the programme a realistic } \\
\text { impression of history. }\end{array}$ \\
\hline $\begin{array}{l}\text { 11. 《汉武大帝》是一部表现形式和内容上都达到 } \\
\text { 相当高度的经典之作, 在再现历史的真实性、欣 } \\
\text { 赏性、艺术探索和追求上达到了一个高度，是此 } \\
\text { 前的历史剧不能企及的。该剧后部分表现的矛盾 } \\
\text { 冲突所反映的思想性极具震撼力, 发人深思。 }\end{array}$ & $\begin{array}{l}\text { The form and content of "The Great Emperor Wu } \\
\text { of Han" matches that of classical works. With } \\
\text { regard to the reproduction of history, it achieves a } \\
\text { high degree of authenticity, appreciation, as well } \\
\text { as artistic exploration and pursuit. It is unmatched } \\
\text { by previous historical dramas. The ideological } \\
\text { quality of the contradictions and conflicts portrayed } \\
\text { in the last part of this drama are strongly rousing } \\
\text { and really set people thinking. }\end{array}$ \\
\hline $\begin{array}{l}\text { 12. 《王保长新篇》很好看, 特别是李保田扮演的 } \\
\text { 那个王保长, 很受我们当地人们的喜爱, 剧中人 } \\
\text { 物的语言很有味道。。. }\end{array}$ & $\begin{array}{l}\text { "The New Work of Wang Baozhang" was great to } \\
\text { watch. We locals were particularly fond of Li } \\
\text { Baotian's acting in the role of Wang Baozhang. } \\
\text { The characters' language had a particular flavour } \\
\text { to it (...) }\end{array}$ \\
\hline $\begin{array}{l}\text { 13. 八套《汗血宝马》不但人物形象鲜明, 很富个 } \\
\text { 性, 导演吴子牛的戏大气, 富人性化。 }\end{array}$ & $\begin{array}{l}\text { Channel Eight's "Hanxue Baoma" did not only } \\
\text { feature distinctive characters with rich } \\
\text { personalities, but director Wu Ziniu's drama was } \\
\text { also atmospheric and rich in humanity. }\end{array}$ \\
\hline
\end{tabular}

\section{Costume Dramas (古装剧)}

\section{Chinese original English translation}

14. '第一剧场' 播出的 《风云 II》, 精彩极了

(这是经本人在全班调查后得出的结果)。

15. 王艳演的《四大名捕》, 真得很好看。
16. 上一期的“周末大放送” 中, 你们重播的《隋
唐英雄传》我很喜欢, 等到它播完的时候, 我就
有一种失落感。
17. 非常喜欢看《上错花桥嫁对郎》, 觉得剧中有
一些黄梅戏的风味。

一些黄梅戏的风味。

18. 最近在 “精品剧场”热播的电视剧非常好看， 这部《怀玉公主》比《还珠格格》还要好看, 我 与朋友都这样认为。我想问的事, 《怀玉公主》 是否有续集? 因为这部剧实在是太好看了, 很轻 松也很幽默。。。让我喜欢的最主要的原因 是：这部剧的几位主演不错, 女主角张得非常漂 亮, 男主角张得很帅。

Clouds Part 2" was truly splendid (I came to this conclusion after having thoroughly investigated the reactions in my class).

Wang Yan's acting in "Si Da Mingbu" is really fantastic to watch.

I really liked "Heroic Tales from the Sui and Tang Dynasties" which you showed during last week's 'great weekend broadcasting'. When the show was over, I really felt a sense of loss. I loved watching "Shang Cuo Hua Qiao Jia Dui Lang" and thought that drama felt a bit like a Huangmei Opera [a type of opera popular in Anhui; author's note].

The popular television dramas broadcasted during the recent 'Fine Works of Theatre' were great to watch. That "Princess Huai Yu" was even better than "Hai Zhu Gege", that's what all of my friends and I think. What I would like to ask is: will there be a sequel to "Princess Huai Yu"? Because that drama is indeed fantastic; very light, and very humorous (...). The main reason why I liked this drama was that the main actors were really good; the characters were incredibly beautiful, the male characters were all very handsome.

\section{Imported Dramas (引进剧)}

\section{Chinese original}

19. 细细的品味, 长今的奋斗精神是能永远感动全 人类的, 不管时代如何变迁, 生命不止甼斗不 息。

\section{English translation}

A delicate taste and continuous spirit of struggle are things that will eternally move the entire human race. No matter how the times change, life has to incessantly be a struggle.

20. 我看了安徽台播的电视剧《特警飞龙》, 实在 是太好看了! 我特别喜欢的是展翼的扮演者洪天 照, 他又帅又酷, 身手又棒, 又勇敢, 我们班理 的许多同学都看过, 都非常喜欢。

I watched the TV drama "Special Unit Flying Dragon". It was really fantastic. I particularly liked Hong Tianzhao, who played the role of Zhang $\mathrm{Yi}$. $\mathrm{He}$ is handsome and cool, agile and courageous. 


\begin{tabular}{|c|c|}
\hline & $\begin{array}{l}\text { Many of my classmates have watched the series, } \\
\text { and they all loved it. }\end{array}$ \\
\hline $\begin{array}{l}\text { 21. 自从看了“正义剧场” 播出的《特警飞龙》 } \\
\text { 后, 我就深深的迷上了这部电视剧, 尤其是武打 } \\
\text { 部分, 惊心刺激, 一件件悬案扑朔迷离, 使我越 } \\
\text { 来越关注《特警飞龙》。我特别喜欢剧中展翼的 } \\
\text { 扮演者洪天照。。。 }\end{array}$ & $\begin{array}{l}\text { Ever since I watched "Special Unit Flying Dragon" } \\
\text { on 'Justice Theatre' I have been a great fan of this } \\
\text { television drama, especially of the fighting } \\
\text { sequences, which are astounding and shocking. } \\
\text { Each one of the legal cases is complex, which got } \\
\text { me more and more hooked to "Special Unit Flying } \\
\text { Dragon". I particularly like Hong Tianzhao in the } \\
\text { role of Zhang Yi. }\end{array}$ \\
\hline
\end{tabular}

Two observations are immediately striking:

1. The viewers' emphasis of the emotional impact that the respective television drama had on them. It is apparently a praise-worthy characteristic of TV dramas to be “moving" (ganren 感人; comment 1), “tear-wrenching" (cui ren lei xia 催人泪下; comment 5), or "rich in humanity" (fu renxinghua 富人性化; comment 13).

2. The majority of comments make strong use of hyperboles. Words or grammatical constructions that suggest "extremeness" or "awesomeness" are repeatedly used (for instance feichang 非常 or tai... le 太... 了; comments 1, 5, 17, 18 and 20).

In addition to these general observations, another more specific feature of these viewer comments is worth mentioning. There seem to be two distinct types of appraisals:

1. The first are comments on main melody themes. These remarks make use of socialist metaphors and tropes, for instance evoking "the party", "the nation", "the people", "the revolution", and "the peasantry" (comments 3, 5 and 8), or referring to a "healthy atmosphere" and content that is "rooted in life" (comments 2 and 4). In general, the authors of such comments tend to emphasise the didactical impact and social significance of the respective dramas.

2. The second are comments on television dramas that might be classified as 'pop' (costume dramas, crime dramas, swordsmen dramas). The remarks on such programmes seem to have been made by young viewers, for instance students who still attend class (specifically comments 14 and 20). The language is colloquial, makes 
use of attributes such as "cool" ( $k$ u酷) or "handsome" (shuai 帅), and primarily points out the appeal that certain actors have had on the viewers.

Although the CUC researchers do not provide any indication of the authors' background or age, the language that is used to describe the two different types of television programmes (i.e. main melody dramas vs. commercial dramas) suggests that they attract two distinct types of viewers: on the one hand those interested in ideologically orthodox mainstream content, and on the other hand those interested in 'pop' content, i.e. action performances, costumes, attractive actors, etc.

In addition to the positive viewer feedbacks, the CUC researchers also present a small number of critical viewer comments, which are summarised in Figure 13 (ibid., 231; author's translation):

Figure 13: Negative Viewer Feedback as Presented by CUC researchers

\begin{tabular}{|c|c|}
\hline Chinese original & English translation \\
\hline $\begin{array}{l}\text { 22. 《任长霞》的纰漏: 第三集任长霞收了群众送 } \\
\text { 来的锦旗后马上招呼同事一起合影, 这与她不爱 } \\
\text { 出名的性格不符。 }\end{array}$ & $\begin{array}{l}\text { A careless mistake in "Ren Changxia": In the third } \\
\text { episode, Ren Changxia receives the silk banner } \\
\text { from the masses and immediately calls everyone } \\
\text { together for a group photo. This doesn't fit the } \\
\text { character of someone who does not wish to be } \\
\text { famous. }\end{array}$ \\
\hline $\begin{array}{l}\text { 23. 对坏人㗊张气焰渲染过多, 过分反映了社会的 } \\
\text { 不安定, 不知海外观众看了作何感想。 }\end{array}$ & $\begin{array}{l}\text { The depiction of the arrogance and fury of the } \\
\text { villains [in "Ren Changxia"] is exaggerated. This } \\
\text { unnecessarily suggests that our society isn't } \\
\text { stable. I wonder if this will not give audiences } \\
\text { overseas the wrong impression. }\end{array}$ \\
\hline $\begin{array}{l}\text { 24. 《完美》中的年轻人正日谈情说爱, 跑酒吧, } \\
\text { 没有进取精神, 剧中的人为了爱情不工作, 不是 } \\
\text { 人生的完美。 }\end{array}$ & $\begin{array}{l}\text { The young people in "Perfect" talk about love all } \\
\text { day long, run off to bars, and don't have any } \\
\text { enterprising conscience at all. The characters in } \\
\text { the drama don't go to work because of love: that is } \\
\text { not a "perfect" life! }\end{array}$ \\
\hline $\begin{array}{l}25 . \text { 《亮剑》错别字太多, 请尽快改正后重播。重 } \\
\text { 大题材重点剧目尤其不可马虎。 }\end{array}$ & $\begin{array}{l}\text { There are too many misspelled Chinese } \\
\text { characters in "Blank Sword"; please quickly correct } \\
\text { this and then rebroadcast the drama. It is really } \\
\text { unacceptable that such a significant key } \\
\text { programme should be treated carelessly. }\end{array}$ \\
\hline $\begin{array}{l}\text { 26. 《抗日名将左权》第 } 15 \text { 集左权与彭德怀谈及 } \\
\text { 多田骏 (冈村宁次) 的军衔时说他 '升为上将' } \\
\text { 是错误的, 日本军衔中三星为大将。 }\end{array}$ & $\begin{array}{l}\text { In episode } 15 \text { of "Famous Adjutants of the Sino- } \\
\text { Japanese War", Peng Dehuai and his adjutant } \\
\text { refer to Tian Jun's military rank and say that he } \\
\text { had risen "to the rank of marshal". This is } \\
\text { incorrect: three stars in the Japanese army } \\
\text { marked the rank of general. }\end{array}$ \\
\hline
\end{tabular}


What is remarkable about these critical audience feedbacks is that the contrast to the appraising comments is relatively stark: the critical remarks appear much more modest, both with regard to quantity and the use of hyperboles. This impression is enhanced by the fact that the negative comments stand in no true opposition to the positive feedback; they do not fundamentally challenge what is being broadcasted on Chinese television. Among the examples above, only one remark truly attacks a television drama in its entirety (comment 24), and the critique in that case is that the drama's content is not ideologically orthodox enough. The other comments are all concerned with detailed errors in certain television dramas, but do not criticise the programmes overall. As the CUC researchers themselves point out, criticism towards television dramas in China is primarily concerned with one of the following three issues (ibid., 231):

\section{Plot mistakes,}

2. Factual errors,

3. Ideological problems (meaning: content that does not conform to $\mathrm{CCP}$ ideology).

The fact that audience feedback has become part of the discourse may be regarded as an indication that the elites are increasingly trying to involve the 'masses' in the creation of China's television content production and broadcasting. However, whether the increase in participation that the CUC researchers have registered in their analysis is in fact a sign that China is generally becoming more pluralistic cannot be sufficiently assessed here; it is in this particular case equally likely that the elites are merely trying to purvey such a pluralistic impression. The selection of feedback excerpts presented by the CUC authors (ibid.) as well as the comments from various interviewees during my own field studies suggest that only certain types of comments (and certain ritualised ways of voicing them) are accepted as 'true' feedback (I3, 121:31 - 128:20; I11, 22:39 - 27:22 ; I12, 45:21 - 46:42; I13, 74:12 - 77:39). 
Furthermore, viewer feedback does not seem to have much influence on the general work of producers and broadcasters: the production crew members interviewed for this research project stated that they did not pay much attention to online debates or viewer comments, and instead relied primarily on the TV ratings to gauge the popularity of a programme (I2, $30: 09-30: 48 ; 16,35: 16-35: 55 ; 113,74: 12-77: 39)$. One reason for this are the issues that viewers raise in their comments: As one interviewee stated, he had repeatedly found that the viewer's discussions in online chat rooms were centred on topics that were very different from his own concerns as a production crew member, and that there was not much to be learned from viewers' concerns with the main actors' performances or the question of which characters they liked best (I12, 45:21 - 46:42). In this sense, viewer feedback is not a serious factor in the creation and broadcasting of television dramas in the PRC.

\subsection{Censorship and Government Regulations}

The production and broadcasting of Chinese television drama content is controlled by diverse mechanisms. The combination of hard institutional controls and soft mechanisms of content management that are adopted in the Chinese context create the need for a broad definition of the term censorship (see Section 2.2.v). Based on these considerations, I define censorship as any authoritative action that aims at actively promoting the construction of certain discursive positions while restraining others, either prior or subsequent to the expression of the discourse fragments.

The following section attempts to explain how censorship in the Chinese television drama sector functions. I first examine which institutions regulate the discursive themes in television dramas (3.5.i). This is followed by an analysis of the forms in which these institutions exert influence on drama creation and distribution, and the stages during which their involvement occurs (3.5.ii). Finally, it is necessary to take into account what guidelines the censoring authorities apply to regulate television content (3.5.iii). 


\subsection{Important Government and Party Institutions}

According to the media experts interviewed for this research project, the main institution responsible for regulating the production, distribution and broadcasting of television drama content at the national level is the SARFT $(13,58: 37-72: 49 ; 14,70: 32-75: 51 ; 15,29: 01-30: 13 ; 16$, $21: 41-25: 01 ; 17,53: 16-55: 06 ; 19,37: 02-38: 21)$. The SARFT is generally charged with all issues relating to broadcasting, including technological issues such as the maintenance and development of the information network, whether through cable, wireless, or satellite transmission. As discussed in Section 2.2.ii, the institution shares certain jurisdiction with the MII, but according to the accounts of the interviewees it is primarily the SARFT and its equivalent bureaus at the provincial level (Guangbo dianshi ju广播电视局) that exert influence on how TV dramas are produced and broadcasted (I2, 54:19 - 55:29; I5, 22:48 - 27:16; I6, $21: 41$ - 25:01; 19, 37:02 - 38:21). Based on official documents provided by the SARFT (2007; author's translation), I have compiled the main eight functions of the institution:

1. Research and formulate general and specific policies on propaganda and the production of radio and television programmes, thereby firmly guiding public opinion. Guide propaganda and the production of radio and television programmes, and coordinate content planning. Guide the structural reforms of radio, film and television.

2. Research and draft the laws and regulations governing the radio, film and television industry. Formulate radio, film and television management regulations, as well as industry development plans. Supervise the management of radio and television programming as well as the programming of satellite television. Supervise the public dissemination of audio-visual programming through the information network. Manage the import of radio and television programmes, and censor content.

3. Examine and approve the establishment and liquidation of radio and television broadcasting agencies, radio and television programmes and television drama production units. Organise the censorship of the content and quality of films, television dramas and other broadcasting programmes. Provide and revoke production and screening permits for films, as well as production and distribution permits for television drama productions.

4. Manage the scientific and technological work in the radio, television, and film sector. Formulate the respective technology policies and 
standards. Guide the usage of high-technology with regard to scientific research and developmental application in the sector of radio, television and film. Research the economic policies relating to radio, film and television.

5. Conduct the concrete planning and management of the dedicated radio and television network in line with overall state planning, macro policy and legal regulations. Formulate the concrete policies, regulations and technical standards regarding the dedicated radio and television network. Guide the construction and development work at the different levels, thereby guaranteeing the safe broadcasting of radio and television programming. By commission of the Ministry of the Information Industries, organise the planning of designated radio and television network frequency bands and manage the channels, power and other technical parameters of the radio and television frequencies. Contribute to the formulation of an overall national information network plan.

6. Provide guidance for China National Radio, China International Radio and China Central Television. Coordinate and inspect these institutions' significant propaganda work. Simultaneously organise and manage the transmission coverage of their programming.

7. Academically formulate regulations pertaining to the radio, film and television system's foreign affairs. Manage and guide radio, film and television matters related to the exchange and cooperation with foreign countries, the Special Administrative Regions of Hong Kong and Macao as well as the Taiwanese territory.

8. Undertake any other tasks assigned by the CCP Central Committee or the State Council.

As the official documents show, the agency influences the development of the television drama sector by approving the establishment and liquidation of production companies, and by granting or revoking television drama production permits as well as distribution permits (ibid.). It further devises censorship guidelines, drafts legislation that governs the television drama sector, oversees censorship procedures at the national level, promotes propaganda, plans what genres should receive priority in future productions and regulates at what time foreign content can be aired (ibid.). ${ }^{63}$ These tasks are divided over eleven departments, which

\footnotetext{
${ }^{63}$ According to one interviewee, the reason that the SARFT restricts foreign content on Chinese television is that the Chinese government feels that the Chinese national identity has to be protected from foreign influences ( 13 , 64:12 - 67:35). Foreign programmes that are belleved to exert particularly high influence in China include series from Korea, Japan and Latin American countries (ibid.).
} 
are presented on the homepage of the SARFT; based on the information provided there, I

have summarised each departments' function in Figure 14 (author's translation). ${ }^{64}$

Figure 14: SARFT Organizational Structure and Main Jurisdictions

\begin{tabular}{|c|c|c|}
\hline $\begin{array}{l}\text { Name } \\
\text { (English) }\end{array}$ & $\begin{array}{l}\text { Name } \\
\text { (Chinese) }\end{array}$ & Description \\
\hline General Office & 办公厅 & $\begin{array}{l}\text { Responsibilities: } \\
\text { - Supervise SARFT decisions, } \\
\text { - Coordinate individual departments, } \\
\text { - Draft policy documents, } \\
\text { - Manage SARFT files, } \\
\text { - Answer petitions, } \\
\text { Sub-Offices: Main Office (办公室), Synergy (综合处), Secretariat (秘书处), Research } \\
\text { (研究处), Finances (财务处), Confidential Network (网络机要处). }\end{array}$ \\
\hline $\begin{array}{l}\text { Department of } \\
\text { Propaganda } \\
\text { Management }\end{array}$ & 宣传管理司 & $\begin{array}{l}\text { Responsibilities: } \\
\text { - Implement propaganda work and art management, } \\
\text { - Assist the SARFT Directorate with respect to the formulation of propaganda } \\
\text { schemes and programmes, } \\
\text { - Coordinate the propaganda efforts of the "three central stations" (CNR, CRI, } \\
\text { CCTV), } \\
\text { - Guide local propaganda efforts, } \\
\text { - Prepare propaganda-related reforms, } \\
\text { - Devise genre plans for radio and television dramas, } \\
\text { - Define propaganda criteria for any audio-visual content, } \\
\text { - Supervise reward ceremonies for broadcasting and television, } \\
\text { - Function as the working body of the SARFT's editorial board. } \\
\text { Sub-Offices: Synergy (综合处), Propaganda (宣传处), Literature and Art (文光处) }\end{array}$ \\
\hline $\begin{array}{l}\text { Department of } \\
\text { Film } \\
\text { Management }\end{array}$ & 电影管理局 & $\begin{array}{l}\text { Responsibilities: } \\
\text { - Formulate policies and regulations for the long-term development of the film } \\
\text { industry, } \\
\text { - Manage production, distribution, and screening, } \\
\text { - Direct and balance film genre plans and annual production plans, } \\
\text { - Censor films, } \\
\text { - Grant and revoke production and screening permits, } \\
\text { - Approve the establishment and liquidation of production units, inter-regional } \\
\text { distribution units, and screening units, } \\
\text { - Manage film technologies, } \\
\text { - Manage international cooperation, such as the import and export of films, or } \\
\text { collaborative film projects, } \\
\text { Sub-Offices: Main Office (办公室), Art (艺术处), Production (制片处), Distribution (定传 } \\
\text { 发行处), International Exchange (国际交流处), Programme Statistics (规划统计处). }\end{array}$ \\
\hline $\begin{array}{l}\text { Department of } \\
\text { Social } \\
\text { Management }\end{array}$ & 社会管理司 & $\begin{array}{l}\text { Responsibilities: } \\
\text { - Formulate a programme concerning the overall arrangement and development of } \\
\text { radio and television broadcasting organizations } \\
\text { - Supervise and inspect regional broadcasting organizations } \\
\text { - Approve the establishment and liquidation of broadcasting organizations, television } \\
\text { programmes, and television drama production units at the county level and above } \\
\text { - Grant and revoke television drama production and distribution permits } \\
\text { - Supervise and manage the public broadcasting of audio-visual material via the } \\
\text { information network } \\
\text { Sub-Offices: Main Office (办公空; charged with NGO management, 民间机构管理处), } \\
\text { Management of Broadcasting Organizations (播出机构管理处), Programme } \\
\text { Management (节目管理处; charged with the management of visual and acoustic } \\
\text { recording, 录音录像制品寞理处), Network Dissemination Management (网络传播管理 } \\
\text { 处). }\end{array}$ \\
\hline $\begin{array}{l}\text { Department of } \\
\text { Personnel and } \\
\text { Education }\end{array}$ & 人事教育司 & $\begin{array}{l}\text { Responsibilities: } \\
\text { - Manage the appointment, dismissal, allocation, examination, reward, punishment, } \\
\text { wages, organization, special duty evaluation, and personal training of cadres, } \\
\text { - Manage the leadership of subordinated agencies, } \\
\text { - Manage subordinated school and university education, } \\
\text { - Promote reforms regarding personnel, education, and administrative management, }\end{array}$ \\
\hline
\end{tabular}

${ }^{64}$ The various web pages that this table is based on can be reached from the following URL:

http://www.sarft.gov.cn/catalogs/zijg/index.html [17.01.08]. 


\begin{tabular}{|c|c|c|}
\hline & & $\begin{array}{l}\text { Direct human resource and qualification management in the radio, film, and } \\
\text { television industry. } \\
\text { Sub-Offices: Main Office (办公室), Cadre Management (干部管理处), Labour and } \\
\text { Capital Allocation (劳资调配处; charged with labour protection, 劳动保护处), Education } \\
\text { (教育处). }\end{array}$ \\
\hline $\begin{array}{l}\text { Department of } \\
\text { Financial } \\
\text { Planning }\end{array}$ & 计划财务司 & $\begin{array}{l}\text { Responsibilities: } \\
\text { - Formulate policies for the development of the radio, film, and television industry, } \\
\text { - Direct financial management in the radio, film, and TV sector, } \\
\text { - Manage SARFT funding and report on financial activities, } \\
\text { - Direct the auditing activities of the SARFT and its subordinate agencies. } \\
\text { Sub-Offices: Main Office (办公室), Financial Affairs (财务处), Project Development (发 } \\
\text { 展计划处), Capital Management and Industry Development (资产管理和产业发展处), } \\
\text { Statistics (统计处; charged with government procurement, 政府采购办公室), } \\
\text { Construction Management (建设管理处), Auditing (审计处). }\end{array}$ \\
\hline $\begin{array}{l}\text { Department of } \\
\text { Science and } \\
\text { Technology }\end{array}$ & 科技司 & $\begin{array}{l}\text { Responsibilities: } \\
\text { - Organise the implementation of long-term science and technology development } \\
\text { - } \text { - Oversee the technological efforts of subordinate agencies, } \\
\text { - Promote technological reforms in the radio, film, and television system, } \\
\text { - Participate in the macro-planning of the national information network, } \\
\text { - Direct the provincial and municipal development of wired broadcasting, } \\
\text { - Manage broadcasting frequencies, } \\
\text { - } \quad \text { Approve all technical aspects of broadcasting at local stations, } \\
\text { - Formulate all policies and plans regarding satellite transmission, } \\
\text { - Supervise and evaluate technological projects relating to radio, film, and television } \\
\text { - Mroadcasting, } \\
\text { - Manage technological norms, patents, measures, and quality control, } \\
\text { Sub-Offices: Main Office (办公室), Science, Technology, and Norm Management (科技 } \\
\text { 与标淮管理处), Wireless Management (无线管理处), Satellite Management (卫里管理 } \\
\text { 处), Wired Network Management (有线网络管理处). }\end{array}$ \\
\hline $\begin{array}{l}\text { Department of } \\
\text { International } \\
\text { Cooperation }\end{array}$ & 国际合作司 & $\begin{array}{l}\text { Responsibilities: } \\
\text { - Carry out radio, film, and television cooperation and exchange with foreign } \\
\text { - Fountries as well as with Hong Kong, Macao, and Taiwan, } \\
\text { - Cormulate foreign affairs regulations regarding radio, film, and television, } \\
\text { - Research broadcasting development outside of the PRC, } \\
\text { - Manage the import of foreign programmes as well as the reception of foreign } \\
\text { satellite stations. } \\
\text { Sub-Offices: Main Office (办公室; responsible for passport and via issues, as well as } \\
\text { for the work of foreign experts, 护照签证处、外国专家工作处), Asia, Africa, and Latin } \\
\text { America (亚非拉处), Europe and America (欧美大处), Hong Kong, Macao, and Taiwan } \\
\text { (港澳台处), Synergy (综合处). }\end{array}$ \\
\hline $\begin{array}{l}\text { Department of } \\
\text { Television } \\
\text { Drama } \\
\text { Management }\end{array}$ & $\begin{array}{l}\text { 电视剧管理 } \\
\text { 司 }\end{array}$ & $\begin{array}{l}\text { Responsibilities: } \\
\text { - Formulate policies and regulations on the production, censorship, and distribution } \\
\text { of television dramas, } \\
\text { - Plan the development of the television drama industry, } \\
\text { - Coordinate the drama genre management at the national level, } \\
\text { - Oversee the day-to-day work of the SARFT censorship and re-examination } \\
\text { - } \text { Committees, } \\
\text { - Mrganise the censorship of state-produced television dramas, } \\
\text { - Grant and revoke television drama distribution permits, } \\
\text { - Regulate drama broadcasting at the national (macro) level, } \\
\text { - Manage national drama award ceremonies. } \\
\text { Sub-Offices: Synergy (综合处; responsible for laws and regulations, 法规处), } \\
\text { Programme Management (规划管理处), Censorship Management (速榲管理处). }\end{array}$ \\
\hline $\begin{array}{l}\text { Legal } \\
\text { Department }\end{array}$ & 法规司 & $\begin{array}{l}\text { Responsibilities: } \\
\text { - Prepare and draft all SARFT legislation, } \\
\text { - Supervise the legal aspects of propaganda and education in the radio, film, and } \\
\text { television system, } \\
\text { - Receive legal suggestions and observe the implementation of SARFT legislation, } \\
\text { - Oversee administrative hearings, redresses, and actions, } \\
\text { - Direct, coordinate, and undertake measures in the radio, film, and television sector } \\
\text { relating to the intellectual property rights and the WTO. }\end{array}$ \\
\hline $\begin{array}{l}\text { Department of } \\
\text { Security }\end{array}$ & 保卫司 & $\begin{array}{l}\text { Responsibilities: } \\
\text { - Direct safety measures in the radio, film, and television sector, } \\
\text { - Manage safety issues at the SARFT and its subordinate institutions, } \\
\text { - Draft regulations regarding public security and fire prevention, } \\
\text { - React to public security or fire emergencies, } \\
\text { - } \quad \text { Assure the protection of inter-departmental secrets and strategies. }\end{array}$ \\
\hline
\end{tabular}


As this figure shows, the main censorship responsibilities are divided primarily over three departments within the SARFT (ibid.): the Department of Social Management (Shehui guanli $s i$ 社会管理司), the Department of Propaganda Management (Xuanchuan guanli si 宣传管理司) and the Department of Television Drama Management (Dianshiju guanli si 电规剧管理司). In addition, the import of foreign dramas and productions that involve foreign participants are handled by the Department of International Cooperation (Guoji hezuo si 国际合作司).65 According to one of the interviewees (14, 60:00 - 70:31), the institution within the SARFT that examines and censors drama content at the central level is the censorship committee (shenchn weiyuanhui 审查委员会). This committee consists of government officials and media experts (directors, university professors, etc.), who assess the appropriateness of programmes in line with the relevant censorship guidelines (ibid.).66

My conversations with the various media experts shows that although national legislation and its interpretation at the central level are, as one interviewee stated, relatively "strict" (yange 严格), regional implementation may vary widely $(15,68: 52-71: 25)$. Such implementation is in the hands of the various Bureaus of Radio and Television that each province, autonomous region or municipality possesses (ibid.).67 The unit within those Bureaus responsible for TV content management is the propaganda department (xuanchuanchu 宣传处; I2, 54:19 - 55:29). This department oversees all stages of TV drama production, from the general plot outline to censorship and final distribution, and is responsible for approving the final product (ibid.). The interpretation of SARFT guidelines at the regional level is extremely subjective: in some cases, production units will submit their drama series to different Bureaus throughout the country, knowing that their product only

\footnotetext{
${ }^{65}$ In addition to the departments (司) presented in this figure, the SARFT also possesses an internal party committee (直局机关党委), a union (总局工会), a unit for retired cadres (离退休斗部局), a disciplinary and supervision group (纪检组、监客局) and a separate audit office (本计局; ibid.).

66 The names of the committee members are publicly available on the following internet page: http://umw.asiatvbiz.com/news/list.asp?id=301 [22.01.07].

${ }^{67}$ For a list of Bureaus, including links to their respective intemet homepages, see: http://muw.sarft.gov.cn/catalogs/zjjg/index.html [22.01.07].
} 
needs to pass the inspection of one institution, irrelevant of how many other agencies may have objected to its content before $(12,55: 30-57: 05)$.

In addition to supervision by the Bureaus of Radio and Television, some productions require additional approval from other state agencies (I9, 38:22 - 38:52): For example, if a drama deals with an issue such as ethnic minorities and education, it will also be reviewed by the respective authorities, i.e. in these cases the State Ethnic Affairs Commission (Guojia minzu shiwu weiyuanhui 国家民族事务委员会) and the Ministry of Education (Jiaoyu bu 教育部; ibid.).

This means that both the production and broadcasting of television drama content is subject to three types of controlling institutions:

1. The propaganda departments of $\mathrm{CCP}$ Committees, which prepare the content of all relevant legislation in China,

2. The state agencies directly responsible for radio, film and television administration, which devise and implement the relevant legislation in line with CCP instructions,

3. State agencies outside of the production and broadcasting sector, but with jurisdiction over certain content matters (i.e. the Ministry of Education, etc).

These three types of institutions exist at all levels of the Chinese nation (i.e. at the national, provincial, district and village level), and the work at the national level is duplicated throughout all tires of the system. The relations in the television content production sector are similar.

What is important to realise is that the inspection and approval of television dramas by a state agency is only relevant to non-governmental production companies; the large state-run units with a general production permit are not censored by state agencies (19, 37:02 - 38:21). Instead, the censorship mechanisms are already integrated into each of these institutions: Production units such as the China Teleplay Production Centre, CCTV or the China Film Group Corporation possess internal Censorship Departments (Shencha bu 审查部) that 
monitor content (ibid.). The cadres responsible for content in these departments are usually former screenwriters, directors or producers who have climbed the administrative hierarchy to receive an influential desk post $(13,52: 16-56: 26)$.

A final institution that exerts macro-control over the TV drama sector is the annual TV Genre Planning Conference (Quanguo dianshiju ticai guihua hui 全国电视剧题材规划会), which is subordinated to the SARFT (I11, 19:19 - 22:38). This conference regulates broadcasting by planning what share of air time shall fall to the different TV genres in the coming year (ibid.). According to one of the interviewees, the purpose of the conference is to prevent the overproduction of one particular type of television drama (ibid.). Another interviewee argued that such regulations are meant to control what is shown during prime time, thereby preventing the largest part of the audiences from being influenced negatively by certain types of dramas, such as crime series $(13,67: 36-72: 49)$. The effect is the same in either case: the conference's decisions provide incentive for the production of certain genres, thereby effectively regulating the market (I11, 19:19 - 22:38).

\section{5.if The Tools Used to Control Television Drama Content}

In general, the censorship process has become increasingly less centralised in recent years. In May 2006, a regulation was passed that now allows local authorities to pass TV dramas for inspection without having to report back (shenbao 申报) to higher agencies; this effort to streamline the administration affects all aspects of content control, from the issuance of permits to the actual censorship process (I11, 16:41 - 19:18). According to one interviewee, it has made the inspection of most TV dramas more of a formality, so that today only programmes that directly deal with party and state traditions (such as revolutionary history dramas) still receive full government attention when it comes to censorship (ibid.).

Nonetheless, my analysis of the interviews suggests that the production and broadcasting of TV drama series is far from being deregulated. According to the interviewees, the main tools 
used by the authorities to control what drama content is produced and aired in China are three types of permits that regulate production and distribution, as well as various broadcasting regulations that function as incentives for producers to create certain contents while avoiding others.

Production permits: Production permits come in two forms $(12,50: 47-52: 11)$ : The first type is the general permit to a production unit (danwei xukezheng 单位许可证), which is issued to large (mostly state-run) production companies and entitles the respective unit to produce any content they see fit $([3,52: 16-56: 26)$. This is made possible by making the respective units responsible for their own internal censorship (ibid.; I9, 37:02 - 38:21). Companies that do not possess such internal censorship mechanisms do not receive general production unit permits, and hence need to register their filming projects with the SARFT or the local Bureau of Radio and Television (12, 52:12 - 52:46;13, 06:33 - 08:43; I4, 37:32 - 43:28; 16, $21: 41$ - 25:01). If the project is approved, the production company will receive the second type of permit: an individual permit for the production of that particular television drama (ibid.). The issuing of these television drama production permits (dianshiju zhizuo xukezheng 电视剧制作许可证) is usually a formality based on the plot outline; according to one interviewee, it used to be a method of assuring proper propaganda content in TV production, but has largely lost that function due to the increased commercialization of China's TV drama market (I4, 37:32 43:28). Today, as another interviewee argued, it serves the government to keep track of the number and types of dramas being produced throughout the country, as well as the financing details of each project (I12, 73:46 - 75:19). The individual production permit is therefore said to have become a filing and recording tool (bei'an 备案), which is intended to prevent an over-saturation of the market with certain types of dramas, as well as the potential duplication of drama concepts (ibid.). In this sense, production is no longer seen as 
a censorship issue by media experts; or as one interviewee put it: "you can film any topic" (shenme ticai dou keyi pai 什么题材都可以拍 $17,57: 47$ - 59:57).

Distribution permits: According to my analysis, the actual process of 'hard' censorship takes place once production is complete and the individual dramas need to be distributed (I4, 60:00 - 70:31): In order to be able to sell a drama, the respective production company must apply for another certificate, the distribution permit (faxing xukezheng 发行许可证). This permit is issued after the final product has been evaluated by the SARFT or one of the Bureaus of Radio and Television (ibid.). If a drama is rejected by the censorship committee, the production company is usually informed how the product needs to be changed in order to be approved.

Broadcasting Regulations: Aside from production and distribution permits, certain broadcasting regulations also influence the production of television drama content, although this influence is exerted more indirectly; the national legislation devised by the SARFT defines at what times during the day certain types of content can be aired by Chinese television stations (I1, 33:14 - 35:18): For example, dramas that feature suspense, horror or crime themes cannot be shown until after 11 p.m. For producers, the regulations on what can be shown during prime time (literally 'the golden time'; huangjin shijian 黄金时间) have a direct effect on their profits (I1, 38:12 - 39:36): prime-time advertisement yields the largest returns for television stations, which means that the stations are willing to pay more for a programme suitable for broadcasting during that time (I5: 80:42 - 82:51). In this way, government decisions on what constitutes suitable television content is relayed via market mechanisms and indirectly affects what is produced in China. A good example is the 2004 national directive that banned the broadcasting of crime series during prime time, which was mentioned by several interviewees (I5: 80:42 - 82:51; 17, 55:07 - 55:57; 110, 42:31 - 49:21): the directive has made the production of crime series unprofitable for most producers, who need 
to sell the rights for re-runs outside of prime time to a large number of broadcasters in order to recoup their expenses.

\subsection{The Guidellnes Governing the Censorship Process}

At the most fundamental level, the censoring of television dramas in China is regulated very precisely by guidelines that are publicly available on the SARFT internet website ( $I 3,48: 37$ 52:15).68 In order to establish what these guidelines are, an analysis of the relevant legislation is first in order.

The policy document most relevant in this context is the "Regulation on Television Drama Censorship Management" (Dianshiju shencha guanli guiding 电视剧龶查管理规定) devised by the SARFT on 7 April 2004 (SARFT, 2004), and implemented on 20 October of that same year (see Appendix 1 for a full translation of this document). The legislation is very clear on who can produce (SARFT, 2004, article 7) and distribute (ibid., article 30) drama content, under what conditions this content may be broadcasted (ibid., article 31), what production units need to do in order to receive the relevant permits (ibid., articles 8 and 21), which state agencies are responsible for censorship (ibid., articles 16, 17, and 18) and how these institutions are supposed to handle the censorship proceedings (ibid., articles 24 and 25). However, when it comes to the topics under restriction, the guidelines are kept abstract, as article 20 in chapter 3 of this document shows (ibid.); the article states that content shall be band if it (author's translation):

1. Stands in opposition to the basic principles of the constitution;

2. Endangers the unity, sovereignty or territorial integrity of the state;

3. Divulges state secrets, endangers state security or harms the honour or interests of the state;

4. Incites ethnic enmity or prejudice, violates ethnic unity or disrespects the local customs of ethnic minorities;

\footnotetext{
${ }^{68}$ The guidelines that the SARFT issues are accessible on: http:/hww.sartt.gov.cn/index.html [22.02.08]
} 
5. Promotes paganism or superstition;

6. Disturbs the social order or violates social stability;

7. Propagates obscenity, gambling or violence, or instigates criminal offences;

8. Insults or humiliates others, or violates others' lawful rights and interests;

9. Endangers social ethics or the excellent cultural traditions of the ethnic minorities;

10. Is prohibited under any other law, or violates any administrative or state regulation.

A likely reason for the vagueness of these criteria is that the authorities wish to retain their flexibility when deciding what content is acceptable or not. The key points therefore leave much room for interpretation.

When confronted with this imprecision of the censorship guidelines, numerous interviewees stated that these vague censorship criteria made the creation of acceptable drama content difficult for them. Three interviewees stated that it was not easy to know what exactly phrases such as "disturb the social order" or "propagate obscenity" were referring to (I2, 53:36 - 54:18; I4, 70:32 - 75:51; I12, 35:35 - 36:48). Other interviewees explained that the detailed concepts that the state and party apply when censoring content is not revealed in official documents, but is instead either derived from many years of experience in the business $(17,57: 47$ - 59:57; 18, 37:52 - 39:05) or from personal contacts to officials (I10, 20:01 21:29). The question then is: what criteria do production crew members themselves apply in order to avoid being censored?

In order to answer this question I asked the interviewees what topics they believed would or should be censored. This is again a matter of perceptions. According to the interviewees, unwanted topics generally fall into two categories (13, 48:37 - 52:15): so-called 'black' content 
(hei 黑), meaning politically sensitive issues, and so-called 'yellow' content (huang 黄), i.e. pornography. 'Yellow' topics primarily refer to the following three issues:

1. Sexuality: Anything that has a sexual connotation (seqing 色情). One interviewee described the limitation of sexual content on TV as follows (18, 39:06 - 40:40): It is possible to produce scenes in which a man and a woman are filmed lying in the same bed; however, the actors need to be under the covers, and no explicit sexual content can be shown (ibid.).

2. Brutality: Anything that is excessively violent (baoli 暴力; $14,70: 32-75: 51$ ). For example, any scene that shows blood is likely to be censored (I10, 42:31 - 49:21).

3. Gambling \& Drugs: In addition to sexuality and violence, 'yellow' content in the widest sense also includes gambling ( $d u$ 赌) and drug abuse ( $d u$ 毒; 18, 83:59 - 86:16). According to one interviewee, these two issues are handled much harsher by the central authorities than by regional agencies (I12, 35:35 - 36:48). The interviewee gave the example of a television drama that he had worked on, which included a sub-plot that dealt with a character having drug-related problems. The production company was instructed by the SARFT to edit-out the entire section, despite the fact that the drama took a critical approach to drug abuse. The interviewee argued that the subplot would probably not have been censored by a regional agency, but that at the national level (the programme was aired on CCTV-8) any type of drug-related issue is likely to be banned.

Note particularly from this last example how it is not sufficient to show unwanted behaviour as deviant, for instance by depicting how a person abusing drugs is punished in the end; the mere depiction of a deviant act already makes it unacceptable to the censors (the reasons for this are discussed in Section 3.6). 
The field of 'black' topics is handled in a similarly subjective way. When asked what issues they considered to be 'black', the various media experts stated that this included content that:

- Attacks the state $(15,29: 01$ - 30:13),

- Criticises the party leadership (I1, 28:58 - 31:46),

- Voices religious opposition (I5, 29:01 - 30:13),

- Supports dissident groups $(11,28: 58$ - 31:46),

- Advocate the independence of certain Chinese territories such as Tibet or Xinjiang (15, 29:01 - 30:13),

- Suggests in any way that Chinese society may be unstable (18, 83:59 86:16).

For this reason, topics such as crime or corruption were viewed as highly sensitive by the interviewees: the argument was that such issues might give viewers the impression that Chinese society or culture was flawed (I7, 65:06 - 70:29), impair the authority of state officials $(14,70: 32-75: 51 ; 19,41: 55-42: 34)$ or inspire malicious viewers to imitate unwanted behaviour and commit crimes similar to the ones shown on TV (these issues are dealt with more comprehensively in Section 3.6). This is, according to one interviewee, the reason why it is not allowed to show on Chinese television how a crime is planned or committed (19, $39: 36-40: 26)$.

Drama content is also required to depict behaviour that does not infringe upon any state regulations. A good example is the subject of driving under the influence of alcohol. In the television drama GAJZ, the main protagonist is repeatedly shown drinking alcohol, and later driving his car home $(113,59: 45$ - 62:11). As one interviewee who had been involved in the creation of that drama argued, this type of behaviour was not yet illegal when the series was produced, and therefore passed the censorship inspection (ibid.). Today, state regulation prohibits driving under the influence, and the same scene would probably not be accepted by the censors again (ibid.). 
As much as contemporary dramas are subjected to censorship, the strongest attention is given to programmes that deal with periods in the PRC's history, i.e. with events that are set after 1949 (I10, 42:31 - 49:21). Events or periods that are directly linked to the legitimacy of the CCP's rule over China, such as the Cultural Revolution, the Hundred Flower Movement, or the so-called Tiananmen Incident, may currently not be adopted into television dramas (I2, 57:06 - 58:16). Periods before the foundation of the PRC are viewed as less problematic (I10, 42:31 - 49:21). The production of historical TV dramas depicting heroic episodes in Chinese history is generally viewed favourably, since such dramas help increase viewers' patriotic sentiment (I5, 90:37 - 92:41). However, historical themes from before 1949 may still cause controversy. For instance, themes from imperial times may be too "feudalistic" (19, 19:43 21:16), i.e. may promote values that are in conflict with Communist ideology. This may, for example, be the case if a feudal ruler is portrayed too positively (see Section 3.7). Also, throughout China's history, themes from the past have time and time again been used by intellectuals to criticise contemporary politics (one need think only of the theatre play Hai Rui Dismissed from Office, Hai Rui ba guan 海瑞罢官, which was one of the factors that started the Cultural Revolution because it was believed to be an allegorical critique of Mao Zedong dismissing General Peng Dehuai from office; Eberstein, forthcoming). The authorities in the PRC are therefore very sensitive to any historical themes that may voice hidden criticism on how China is currently ruled. To account for the importance of such historical themes in television dramas, the SARFT has charged a special working group with the supervision of such content: the "Small Leadership Group Concerning the Creation of Main Historical and Revolutionary TV Genres" (Zhongda geming he lishi ticai yingshi chuanzuo lingdao xinozu 重大革 命和历史题材影视创作领导小组; 19, 37:02 - 38:21).

According to the interviewees, all of the above issues are considered by the responsible censorship organization before it will issue a distribution permit; if the television drama 
under examination does not fulfil the censors' criteria, it will be sent back to the production company with notes on how the programme needs to be amended $(14,60: 00-70: 31 ; 15,27: 17$ - 29:00). This process is described in the relevant legislation (SARFT, 2004, article 24). In line with these official guidelines, producers have the right to object to censorship through the reexamination committee (dianshiju fushen weiyuanhui 电视剧复审委员会) at the SARFT (ibid. article 25). If a drama has been approved by the censors, it may not in any way be altered (ibid., article 26). Furthermore, the SARFT is authorised to "order the revision of a television drama or the suspension of its distribution, import, export or broadcasting even after the programme in question has been issued a 'Television Drama (Television Cartoon)

Distribution Permits', if the public interest calls for such actions"' (ibid., article 32).

According to one interviewee, there are three reasons why the authorities may change their minds about a TV drama after it has been cleared for distribution (15, 32:28 - 35:41):

1. Officials may follow the audiences' reactions and discussions on the internet and be discontented with the feedback.

2. Officials may receive letters from viewers voicing discontent.

3. Renowned public figures (politicians, intellectuals, etc.) may draw attention to a TV drama's alleged inadequacy by publishing articles in the papers or on the internet (this is the most common case).

Two examples illustrate this type of belated censorship (I5, 30:14 - 32:27):

1. In 2001, the Taiwanese teen drama Meteor Garden (Liuxing huayuan 流星 花园) was approved for broadcasting in the PRC and was partially aired. However, broadcasting was suspended when it became clear that the programme was becoming too popular with young audiences. The authorities feared that the hedonistic content might have a bad influence on such a large number of young viewers.

2. In 2005, Chen Kaige's TV drama adaptation of the Romance of the Three Kingdoms (Sanguo yianyi 三国演义) passed the censors, but later had to be edited because the authorities changed their mind about the literary accuracy of the adaptation. 
In general, however, TV programmes are rarely edited or banned once broadcasting has commenced $(I 5,32: 28$ - 35:41). One interviewee argued that the authorities had realised that it draws far more attention to a TV drama and its content if the series is cancelled, than if it is allowed to continue; the officials fear, so the argument goes, that viewers will only be more interested in a banned programme and will watch the series on pirated DVDs or on the internet (ibid.).

Even though the ambivalence of China's censorship criteria may at times cause problems for production crew members, and some programmes may even be banned after they have initially been approved, the producers of television dramas only rarely test the limits of what can be created (15, 30:14 - 32:27). As the interviewees explained, the intention of producers is to distribute their product, and they know what topics to steer clear of if they wish to achieve this goal (112, 35:35 - 36:48), or in the words of one interviewee: "none of us would think of producing anything about Falun Gong" (women kending bu yao zuo Fanlun Gong de dongxi 我们] 肯定不要做法轮功的东西; I7, 53:16 - 55:06).

\subsection{The Chinese Perspective on the Influence of Television}

After having examined to what extent and with what methods the state and party control television content, the following chapter analyses what opinions officials and media experts hold towards television entertainment. The intention is to show how these opinions influence the production, control and broadcasting of popular television dramas. To this end, the following two sections ( 3.6 and 3.7 ) both attempt to identify recurring themes (tropes and metaphors) in the way that Chinese media experts and officials talk about the subject of television drama in the interviews conducted for this research. My analysis of these perceptions shows that what is held to be true about the media, its functions and its influences on consumers is a crucial variable in the production of television content. Hence an understanding of media experts' opinions on the subject is essential in order to 
understand why television dramas are produced the way they are in China, and what social relevance they have.

Chinese media experts are divided over the question how much influence the media exerts over audiences. Discursive positions range from the notion that viewers are essentially passive recipients who readily adopt the views presented on television, to the opinion that television is merely one of many factors that influences people's behaviour. The following section presents these different positions. It addresses the question of how influential television content is believed to be (3.6.i and 3.6.v), what effect it may have on viewers (3.6.ii), which types of viewer are regarded as particularly prone to such effects (3.6.iii), and how television's potential influence is used to justify the government's control of the media (3.6.iv).

\section{6.i The Official Position: Media Content's Impact on Viewers}

In the Chinese debate on television's power of influence, the viewers are mainly regarded as susceptible to television content. For instance, the political leadership attributes a very high degree of influence to television in general, and to television dramas in particular (I1, 36:31 $38: 11 ; 13,48: 37$ - 52:15). So before turning to the opinions of media experts on the subject, a look at the official position is in order. The faith that officials place in media content as a tool to influence the public, as well as the fear that this potential influence simultaneously instils in the leadership, can be best demonstrated by quoting from the rules, regulations and decrees concerning television and film. The following three excerpts each feature at least one of the popular tropes used in official statements on media influence (author's translation):

1. The censorship of television dramas should insist on serving the people and serving socialism, should conform to the principle of 'letting a hundred flowers bloom and a hundred schools of thought contend,' should be close to reality, close to life and close to the principles of the people, and should guarantee the correct guidance of public opinion. 
电视剧审查应当坚持为人民服务、为社会主义服务的方向和白花齐放、白家争 鸣的方针，坚持贴近实际、贴近生活、贴近群众的原则，确保正确的舆论导 向。(SARFT, 2004, article 19)

2. The Bureaus of Radio and Television at all levels should positively guide and encourage film and television programme production organizations to produce film and television dramas that are suitable for web broadcasting and that feature positive and healthy content.

各级广电局要积极引导并鼓励影视节目制作机构多生产适合在互联网上传播的 内容积极健康的影视剧。(SARFT, 2007)

3. The state encourages the creation of excellent films that unify ideological quality, artistic quality and viewer appeal; are close to life, close to reality and close to the masses, and protect and benefit the healthy development of minors. (Films) should vigorously develop and advance culture, sustain a healthy and beneficial culture, work hard to reform backward culture and firmly resist any degenerate culture.

国家提但创作思想性、艺术性、观赏性统一，贴近实际、贴近生活、贴近群 众, 有利于保护未成年人健康成长的优秀电影。大力发展先进文化, 支持健康 有益文化，努力改造落后文化，坚决抵制腐朽文化。(SARFT，2006，article 22)

I shall return to these statements throughout this chapter in order to establish how such official positions relate to the perceptions of the media experts interviewed for this project. To start with, the first trope is that the media guides public opinion (yulun daoxiang 奥论导向), which is used in example one. Another closely related trope can be found in example three: the idea that media content advances, develops, sustains or reforms "culture" (wenhua 文化), and has the potential to resist or repel "degenerate culture" (fuxiu wenhua 腐枌文化). Both these tropes are evidence of the influence and even constructivist potential that the media possesses according to the official Chinese position. What exactly the public is meant to be led towards, or what kind of culture is meant to be created, remains unanswered in the text examples; the SARFT merely demands "correct" (zhengque 正.确) guidance and "healthy" (jiankang 健康) culture.

This brings us to another recurring theme used in Chinese media discussions: the metaphor of 'healthy' content, which is employed both in examples two and three. The health- 
metaphor is not only used throughout official Chinese documents, but also by media experts themselves. As an example, consider the following interview excerpt $(19,18: 27$ - 18:48):

R: How does one know what counts as good didactical content, because this is also debatable, right? The director, the creative producer, and the screenwriter all have to decide what to film, so...

19: My position is this: a television drama, and I am not talking about communism, socialism, the communist party, I am not talking about any of those things. But there is such a thing as a base line. So what is that base line? You have to help promote people's mental and physical health and the developmental progress of this society.

I will return to the concepts of progress and development that the interviewee mentions below. For now, it is noteworthy that terms like "health" or "healthy" were used repeatedly with regard to television content by various interview subjects $(19,19: 43$ - 21:16; I10, $21: 30$ 22:29; $111,34: 41$ - 35:08; I12, 40:34 - 42:02). The implication is that media content literally has a physical and spiritual effect on the audience's wellbeing. A similar argument is made in text example 3 above, which attests that the content of films has the potential to "benefit the healthy development of minors"(SARFT, 2006, article 22). It also suggests that media content can potentially harm the audience, and that particularly young viewers need to be "protected" (baohu 保护; ibid.). This is the fourth important recurring theme: the idea that the state has a responsibility to protect its citizens from harmful media content (I2, 58:17 - 60:38; $13,48: 37$ - 52:15; I6, 25:02 - 26:52).

The question then is: what kind of harmful content do the Chinese elites feel they need to protect the audience from, and what effects are such harmful elements believed to have?

\subsection{Possible Effects of Harmful Content}

There are four recurring arguments that are used by media experts to describe what harmful effect television content may have on viewers and on society. The first two of these arguments are made in the following interview segment (I7, 65:37 - 67:10): 
R: Currently, many television dramas discuss the problem of corruption. Which is important, but also probably a bit complicated. If too much criticism is expressed, that wouldn't be good either. So how do you choose...

I7: In the past couple of years such dramas were quite common, but now they have become relatively rare. Because television dramas that deal with corruption are, as a matter of fact, what we call a double-edged sword, which is unlike a knife. A knife normally has one edge that cuts, and one edge that doesn't. But swords have two edges. At least Chinese swords do, I don't know about Western swords. So both sides of a sword can cut, they are both sharp. It has two sharp edges. That is a double-edged sword. So when you show corruption or the fight against corruption to the people, then at the same time it might have an adverse effect. It may cause people to think: "wow, such corruption", and instead this will stir up factors that make society unstable. For example, maybe some of the crime dramas we just talked about deal with public servants and police officers solving a case. They can't help but also show how policemen solve the case and how criminals commit crimes. Actually, in some cases, in foreign countries as much as in China, someone might get caught for a crime and then say: "I learned that from TV." That is to say, there are many precedents were someone learned (such methods) from TV dramas. That's why, as far as possible, we need to be cautious when dealing with such sensitive topics.

In this excerpt, the interviewee states that criminal behaviour to some extent results from viewers emulating bad behaviour shown on television, and that malicious viewers may copy the criminal methods shown in television shows; an argument which is also made in I1, 33:14 - 35:18 and 19, 39:36 - 40:26. A similar logic fuels the arguments against violence, sex and drugs on television: as mentioned in Section 3.5, the fear is that audiences might be inspired to copy such behaviour. This idea that people emulate behaviour is a core belief in China's communist ideology. Ample proof of this is the repeated evocation of model workers, peasants and soldiers by the party and government since the establishment of the People's Republic (Thoegersen, forthcoming) ${ }^{69}$ It should, however, be noted that the importance of role models is not a communist novelty. Much of traditional Chinese thought is built on the

\footnotetext{
${ }^{68}$ One of the most famous of these role models is the legendary communist martyr Lei Feng (战䗇); for an evaluation of Lei Feng's relevance in post-Mao China see Zhang (1999).
} 
axiom that the individual emulates the actions of others, and that for this reason the sage has to set a positive example for others to follow.70

Whereas this first argument is mostly linked to social concerns (i.e. a possible increase in criminal behaviour, drug use, sexual misconduct, etc.), the second argument is concerned more with legitimacy. Consider again the above interview segment: The interviewee explains that displaying the morally wrong conduct of officials may give viewers the impression that their government is unstable. This, in turn, may undermine the authority of the party and government. Very similar statements were also made by other interviewees (14, 70:32 - 75:51; I9, 41:55 - 42:34).

The third argument is concerned with the depiction of historical events and figures on television. According to this line of argumentation, historical television dramas may cause viewer to adopt unwanted attitudes towards Chinese history. This may in some cases mean that the authorities are worried the audience will reject the official position of party and state towards history in general and the nation's progress under party rule in particular (19, 19:43 21:16):

19: Like the drama series $H a n W u D a D i$, why was it criticised? It's historical perspective was problematic. The Emperor Wu of Han... such a prominent individual existed in history, there's nothing wrong with that. But history was not about this one person alone. If you now say that Emperor Wu of Han was a brilliant authority, brilliant and great and perfect... a perfect individual (...), then what about the historical perspective? He has to have been a feudalistic emperor, so he had many bad aspects.

R: Right, so the drama didn't point out these bad aspects.

19: Exactly. So it's like this: in the title song, the director states that she wants to treat him as a model of brilliant authority. So what is wrong with that? I's wrong. Think about it: if an emperor is good, and we admire the emperor that much... what do we still want with democracy then? What do we still want with republicanism? Do we Chinese people

\footnotetext{
${ }^{70}$ Donald Munro (1969) shows this in his research on the relevance of virtue (de ${ }^{\prime}$ ) in early Chinese society. He writes: "In the Western Zhou, ancestors were frequently cited as models whose de must be emulated. (...) Eventually, living men who embodied the norms also came to be regarded as models" (ibid., 190).
} 
still want to progress forward? We don't want to go down the road of the imperial age again, right? This then is the negative influence of this drama: Culturally speaking it promotes backward ideas. If you teach that this emperor was great, if you admire that emperor... we can admire him, but historically I look at his function, look at his contribution, and I subject him to a political evaluation. So if today he is still great, if we still call out "forever live the emperor," then what hope do we have? Can this society of ours still progress? What do our people still need democracy for?

The argument here is that the PRC is a republic and a democracy, progressing forward through history, and that the positive depiction of feudalistic times would cause China to regress into a dark age. This, again, is an important trope: the progress that Chinese society has achieved needs to be protected against backward-oriented forces. This perceived potential to negate historical progress explains why the government places such a high priority on the control of historical television series (I11, 11:18 - 14:19; see also section 3.5.iii). One example of a popular television series that received harsh criticism for depicting unacceptable historical content is the drama Towards the Republic (Zouxiang gonghe 走向共和; 2003). The programme received so much disapproval for its allegedly uncritical portrayal of the historical figures Li Hongzhang, Yuan Shikai, and Empress Dowager Cixi (I9, 33:50 35:34) that CCTV was forced to accelerate the broadcasting of the fifty-nine-episode series in order to remove the programme from the air as fast as possible, without simultaneously drawing unwanted attention to the series, for instance by banning it $(13,81: 21-84: 36)$.

At first sight it may seem that this argument is again related to the problem of legitimacy, and that the depiction of pre-communist periods might call party and state politics into question. However, the fear of "feudalistic trash" (fengjian zaopo 封建槽粕; I3, 87:23 - 91:00) is not merely a propaganda issue; it also has a didactical dimension that goes beyond questions of state and party legitimacy. The concern is that the portrayal of certain role models may cause viewers to accept outdated social concepts, for example by adopting the patriarchal attitudes of past centuries and rejecting gender equality (ibid.). In this context, some experts 
demand a more socially responsible (i.e. didactical) approach to TV drama, as well as

additional 'healthy' TV education on different aspects of Chinese history and traditional

thought, such as offered by CCTV's education programme Lecture Room (Baijia jiangtan 百家

讲坛; ibid.).

This then is the fourth effect that media experts in China associate with potentially harmful

television content: the distortion and decay of Chinese values. The following, slightly longer

interview excerpt offers two such examples (13, 94:12 - 97:24):

I3: Well, for example, Korean television dramas are broadcasted quite a lot, but they are not sensible about ethnical culture. You personally wouldn't think, for example, that "Confucianism* was Korean, yet some people think it originated in Korea.

R: (laughs) really?

13: Yeah yeah yeah! This is currently propagated in South Korea. So some people will think that these early Confucianists were born in Korea. Such problematic opinions are being voiced. So under such circumstances, television's influence on the masses really can't be ignored. Well, whether or not the masses have a particularly strong kind of discriminatory power, a capacity an *ability* to choose, that has to do with age, knowledge, background, with each person's *educational background ${ }^{\star} . .$. it has to do with all these aspects. Among young people whose character isn't fully developed yet, this ability is still weak. I think if we conducted a sociological or psychological survey, and this is of course my slightly subjective view, but $I$ think if we conducted a survey in... what is it called... "cultural psychology", we would find that such influences exist. So under these circumstances moderate restrictions and protections are still required. "I think it's necessary*. We can't say it doesn't matter, just broadcast whatever you want to broadcast, including those black and yellow and ethical issues we talked about earlier. One of my students... what was it she said? She said: "ma'am, do you know what we currently..." "These are eighteen, nineteen year old kids, and I was asking them: "What are your criteria for finding a partner? "how do you choose your boyfriend or girlfriend", what is your "criterion*. They said something that almost made me die laughing. They said "with car and apartment, no mother no father."

R: I've heard that, yeah.

I3: You've heard that, right? (laughs)

R: Right, right, I've heard that. 
13: I said that from an ethical perspective this is immoral. Really terrible! "With car and apartment, both parents deceased." That means the parents are both dead. After that I said: "not only is that unethical, but at the same time it is also extremely" ... what was it I said to them... "also extremely deranged, extremely unwise." By unwise I mean that that's really stupid. "Foolish"! Extremely "foolish". If the boyfriend's parents pass away while he is still that young, then don't they have this... what is it called? *Heritage", these genes, this genetic...

R: Oh, you mean *genetic*.

I3: Yeah yeah yeah: "genetic*. There's a genetic factor, an *influence*. Well, will this *boyfriend" then not also die very young? Do you understand?

R: I understand, I understand (laughs). I understand.

I3: This is really an extremely *foolish* thing. How can you not want your *parents* to have a long life?

R: Old age.

I3: Right! (R: laughs) That is to say they have an extremely terrible moral perspective (R: hm). Extremely terrible.

I have reproduced this interview passage in full because it demonstrates that the call for stricter media control is fuelled not so much by a threat to state and party legitimacy, but by genuine concerns about the erosion of social values in China. In the above excerpt, the interviewee is worried that:

1. Chinese audiences may forget their historical heritage (in this case believing that Confucianism originated in Korea), and that

2. The moral values of young viewers are becoming increasingly deranged.

Such concerns are sometimes voiced towards family dramas $(13,69: 54$ - 72:20), but typically also towards teen soaps, which by default target a young audience, and mostly depict stories of consumption and economic success (I11, 60:19 - 64:44). For this reason, some critics refer to this particular genre of television series as "vomit drama" (outuju 呕吐剧, a play on the Chinese term for "teen drama", ouxiangju 偶像剧; ibid.). An example of a teen drama series that was banned from television after broadcasting had started is the Taiwanese telenovela 
Meteor Garden (Liuxing huayuan 流星花园; 19, 40:27 - 41:54) that was mentioned above:

parents complained that their children were being corrupted by the programme's hedonistic

depictions of adolescences; the critique was that the series' endorsement of consumption,

parties and fights with other teenagers caused young viewers to act in similar fashions,

thereby encouraging them to neglect their school education (ibid.).

\section{6.iif Risk Groups}

The above description shows that one group of viewers is believed to be particularly

susceptible to the potential negative effects of television: Chinese youths. As stated earlier, the government feels that the media should promote "the healthy development of minors" (SARFT 2006, article 22). This view is shared by Chinese media experts. The rationalization that underpins the call for media control for the sake of China's young viewers is explained by one interviewee as follows (13, 93:01 - 94:11):

13: Well, in my opinion, the influence of television is different for people of different ages. For young people, the influence is... their mental age is different, their physiological age is different, each person's attitude towards outside influences is different. For example on teens, what are they called...

R: *Teenagers*

13: Right, *teenagers", and this... what is that called: *ad...*

R: $\quad$ *Adolescence*

13: Yeah, "adolescence*. People of that age, such kids, well they can be spoiled quite easily. (...) So for the sake of those people, I really feel television dramas should have a ratings system. That is something that the entire world is demanding. With regards to this "art form", everyone is considering this problem. Adults, middle-aged people, and those who are of relatively mature age, they all already possess the ability to *choose* and "judge*, they possess their own value system. They won't be so easily subjected to outside influences. I mean, influence or no influence, maybe they are influenced as well. But these people may possess stronger judgement, whereas children of young age may be influenced more strongly. 
The concern for adolescents is often linked to the argument that China does not possess a system of parental guidance, and consequently requires the government to control what is aired. When asked why the Chinese guidelines were stricter than those adopted in other countries, one interviewee answered: "well, that is because we do not have a parental guidance system” (na, jiu shi yinwei meiyou fenji zhidu 那，就是因为没有分级制度“; 19, 38:53 39:09). Another interviewee emphasised the relevance of such a guidance system the following way (I7, 67:11 - 67:50):

17: Because to this day China does not have a system of parental guidance for film and television. It would seem that foreign countries all have a very clear parental guidance system.

R: Yes, right. Right.

I7: There, anyone accompanied by an adult can watch a programme, and otherwise only those who are older than 18 may watch it. For many years, China has developed in a way that now everyone watches television, it is a type of universal entertainment, a type of universal consumption. And in addition, a household may have only one television set or two television sets, so that everyone at home, whether young or old, all watch the same programme.

Teenagers are certainly a major group believed to be at risk when it comes to television's harmful effects. However, the concept of the passive audience, and hence of the susceptible viewer, is sometimes extended from young people to the broad 'masses' in general (dnzhong 大众). This is again a common trope in the Chinese discussion on media influences: the idea that, as one interviewee put it, the audience has "varying levels of education, morality, and culture" (butong de jiaoyu zhidu, daode shuiping, wenhua suzhi 不同的教育程度、道德水平、文 化素质; I6, 40:22 - 43:05), and that the average viewer is generally uneducated (I2, 38:01 $39: 28 ; 13,115: 34-121: 30 ; 112,46: 43-49: 43 ; I 14,46: 49-47: 43)$. This trope is closely linked to the perceived unique economic and social level of development in China. It is therefore often used to explain other social shortcomings in the PRC as well. A good example is the 
following interview segment, in which the interviewee argues that the public conduct of most Chinese citizens demonstrates their inability to act responsibly $(14,66: 38$ - 67:53):

I4: Because at the moment our society's development has not yet reached this level. We don't have an effective level of supervision. It's not like China didn't have any laws, but the common Chinese people's legal consciousness has not yet reached this level. Their knowledge of these laws has not yet reached this level. They do not yet possess the consciousness and the mentality. Their cultural quality, to speak plainly, has not reached this level. Take, for example, standing in line when getting on a bus. Everyone knows that is a very good thing to do. Yet some people don't abide by this rule. So, when one person does not abide by this rule, then others will also not follow it. But when people don't abide by such a rule, there's no way to punish that. So it just becomes a chaotic situation. However, if the common people, if we all stood in line on our own initiative like foreigners do... I don't need to tell you this, right? So that's why I think this is not an institutional problem, but one of transforming the quality of the citizens.

The trope of the uneducated masses is used by intellectuals of all political orientation in China: both those arguing the socialist position (14, 60:00 - 70:31) as well as those demanding more political participation (114, 12:37 - 16:17) point out that the masses require the guidance of the more educated. The reason why the same trope is used in both cases is that the idea of an educated and benevolent elite governing an uneducated and underdeveloped society is not rooted primarily in contemporary political culture, but in much older traditional Chinese thought (Pye, forthcoming).

\section{6.iv Justlfying Censorship and Govermment Control in China}

The perception that certain social groups in China are particularly affected by 'unhealthy' media content is central to the justification of government censorship and media control. The first form of justification has already been mentioned above: it is the wish to protect China's youth from harmful media effects, particularly in the absence of a parental guidance system. However, the lack of a parental guidance system is not the main argument that is used for justifying government censorship. Instead, it is the perception that the systems which are 
enforced in other countries would not suit the Chinese context, and that the government

therefore has no choice but to supervise media consumption $(14,65: 33$ - 66:37):

I4: So why do you need to pay attention to this issue? Because at the moment China does not possess a system of parental guidance. This is a real problem. We don't rate our television dramas and films. We haven't rated any of them yet. And shouldn't they be rated? Once social development has reached a certain level, we can rate them. Parental guidance is also related to another problem, that of supervision. Right? That is to say if you don't have supervision and you rate films, it's as if you hadn't rated them at all. Do you understand what I'm saying?

R: I understand, I understand.

I4: For example if I classify, let's say, dramas with sex and violence as content that can't be broadcasted at a certain time.

R: So that only children older than $12 \ldots$

I4: Right, right, right. The cultural quality of your common Chinese household still hasn't reached that level. If the requirement for a programme is that only viewers over $\mathbf{2 0}$ may watch it, but my child is a teenager, and I'm not at home that day, then if my child watches that programme, what can I do about that? So this type of supervision, including cinema supervision... for example a certain movie is only suitable for children over the age of 13 , and others shouldn't watch it. Well, say the head of a household goes out with his child and buys a ticket for that film, then the cinema personnel is thinking "one more ticket won't make a difference", so they sell him the ticket. If you can't enforce supervision, such a parental guidance system is useless. Right? I think that's the way to look at it. There's nothing else we can do. (laughs)

The second justification draws from the trope of the uneducated average citizen mentioned earlier. To evoke the image of a socially underdeveloped China populated by uneducated 'masses' serves a specific function: it justifies a paternalistic attitude, according to which the Chinese people (renmin 人民) require guidance and protection by the elites (Heberer, forthcoming). The argument for media control is in that sense not so much driven by the concern that the wrong content might contest China's one party rule or otherwise facilitate political dissent (although this is certainly one aspect of media control in the PRC, as the official censorship guidelines show) but is instead fuelled by the perception that the Chinese elites have a responsibility to guide the uneducated masses towards welfare. 
The concern for China's welfare is closely linked to the third justification of media control: the idea that certain television content may endanger social stability (shehui wending 社会稳 定). The stability trope is central to the Chinese media debate. Consider the following excerpt, in which an interviewee explains why certain periods in recent Chinese history are still taboo (I14, 09:27 - 09:53):

R: And what other things can't be broadcasted, or are prohibited?

I14: Prohibited? It's like this: each period of time is different. For example, during this current period you can't talk about the ten years of the Cultural Revolution. So dramas on the Cultural Revolution should best not be produced. Because that's a sensitive issue. Doesn't the government say... they only use one word: stability. Anything that isn't *stable* is not alright. So that's why they say "Cultural Revolution dramas can be produced next year. They can be aired next year." That is precisely because of stability.

The reason why social stability has such a high priority in Chinese discourses is that it is associated with economic development. Without social stability, so the argument goes, China cannot prosper (14, 72:42 - 73:51):

14: I think with regard to the government... why will the government consider the question of officials' authority? Because when we talk about Chinese society, currently the most important thing is stability. Social stability. Because only if this society is stable can the economy develop. This is the government's reasoning. Because we currently witness that, in many countries, if the government and society aren't stable, the economy has no way to develop. For example Yugoslavia.

R: The economy doesn't work.

I4: The economy doesn't work. Nothing works. So that is why I think that especially with a country of this size, society's stability is very important. Because only with social stability can the economy develop. If society isn't stable, the danger to the economy is huge. Huge. So I think our government is looking at it from that angle. It will restrict you from broadcasting on television and through the media any content that threatens the stability of society. The government needs to regulate the situation.

Finally, the advocates of media control in China argue that Western countries censor television content as well, particularly when it comes to themes such as violence, sexuality 
and drug abuse (I6; 25:02 - 26:52; I9, 22:25 - 23:34). Despite the political, social and cultural uniqueness that is so often claimed for China, the references to other country's censorship guidelines are an attempt to equate the principle concerns on which censorship in the PRC is founded with the principles that are believed to apply in Western countries. Furthermore, the fact that television dramas are rarely rejected entirely by the censors but are instead sent back to the producer with the option to re-edit the programme was viewed by one interviewee as proof that foreign criticism of the Chinese censorship system was unjustified: censorship was only seen as unjust by the interviewee if it meant that a finished product was completely banned (14, 60:00 - 70:31).

\section{6.v Putting the Official Position into Perspective: Soclety and the Viewers}

The debate on television's influence and on media control that has been discussed so far can be viewed as hegemonic discourse in the sense that it is adopted by the Chinese leadership (i.e. in the official discourse) and frequently reiterated by media experts. However, this is not to say that this dominant viewpoint is not contested by diverging positions. Particularly the level to which television is often believed to influence audiences is questioned to some degree. Consider the following interview segment, in which the interviewee hypothetically quantifies television's impact on viewers in comparison to other factors of influence $(17,70: 30$ - 72:06):

R: I would like to ask you about your personal opinion in the following matter: what kind of influence does television have?

I7: I think it's quite... you say it has an influence, and it's possible that this influence... if we're talking about quantity, and of course I have not done a survey, but it shouldn't exceed ten per cent.

R: Ten per cent.

I7: No more than ten per cent. A larger part, maybe ninety per cent, is society's influence. Because the influence of television and the media, and the media is not only television but includes television as well as the internet and possibly other things, the media's influence won't exceed ten per cent. More important is the influence from society and the family. 
It's the influence of the people they come into contact with, of the family they live in, of the work they do, and of their living environment that influences people. However maybe some person on some occasion, just like we just said, they might commit a murder. That may be a problem caused by social reasons, but that person learned the method from television or the internet. So he suddenly had a mental flash (on how to commit a crime), but what really influenced him is not television at all.

Particularly noteworthy is that this statement still makes use of the trope that criminals potentially learn the methodology of crime from the media. The interviewee acknowledges this dominant position, but suggests that the reasoning behind it (i.e. the conception that television is the cause of criminal behaviour) has to be put into perspective. The use of hegemonic discourse fragments in counter-hegemonic arguments becomes even more evident in the following excerpt (112, 36:48 - 39:32):

R: What function do you yourself believe television and television dramas have? What influence might they have on audiences?

I12: Actually, I believe that in many cases their influence is exaggerated.

R: may be exaggerated...

I12: Their influence may be exaggerated. Because, I believe it's a bit like... I have a little child myself. My child is still very small.

R: Really?

112: Sometimes I worry that my child might learn this or might learn that... I worry a lot. Well, in reality many of these worries are unnecessary. Because your little child may find its own way to grow up. It has its own way to distinguish things. But grown-ups will always be fathers. Parents always want to keep their children away from harm. They don't want them to see this, don't want them to see that, don't want them to encounter any danger, don't want them to encounter any harm. Parents probably are in such a state of mind. I sometimes think that these officials probably also have such a mentality.

R: Oh, really? Like parents? (laughs)

I12: Yeah yeah yeah! They think "I'm responsible, I have the duty to help people choose." That's the type of mentality that parents have. And I am not saying that such a mentality is wrong, but such an approach is not very scientific. It's not an entirely scientific approach. Sometimes these worries may be excessive. It's that kind of choice, that kind of attitude that they may exaggerate. They may think that the function of television and of television dramas... well, there are still people who watch a 
kidnapping on television and, well, imitate that and commit a similar kidnapping. Also, there may still be people who watch something on television concerning some historical period and then think that that historical period was exactly like shown on television. That sort of situation may nonetheless occur. But this will normally be something that a very disturbed person will do. A very peculiar person might act in such a fashion. A peculiar person may have such notions. He may be completely consumed by television. But most sane people will not be consumed by television. This is my personal opinion, but maybe those who work for an administrative agency feel they have some kind of responsibility to filter out anything that might consume the audience, so they won't allow you to show too many historical details, or crime, or drug abuse. They fear that people may imitate those things. I think that's like the excessive worries that parents have towards their children.

In the example above, the interviewee uses the traditional trope of the paternalist government and turns it against the hegemonic position: the argument here is that the government indeed feels it needs to protect its citizens like parents protect their children, but that the government's concerns consequently are similarly exaggerated as those of parents.

It is important to note, however, that in both of the above counter-hegemonic statements media control is not outright rejected. The importance of social influences is emphasised, but the potential of television or other media forms to inspire malicious behaviour are nonetheless acknowledged in both excerpts. This demonstrates how even discursive positions that challenge the dominant conceptions of the media's influence on consumers are still confined by the boundaries of the hegemonic discourse (additional examples are 13, 91:01 - 98:03 as well as $17,65: 06-70: 29)$.

\subsection{The Chinese Perspective on the Functions of Television}

The degree to which Chinese media experts believe television influences viewers' behaviour raises the question what functions television drama is supposed to have. These main functions that are attributed to popular television series in official statements are propaganda, education and entertainment $(15,56: 53$ - 58:15). In addition, television is also believed by some to serve as a form of social and psychological release (17, 72:15 - 73:26). The following section analyses what interviewees associated with these functions. 


\section{7.i The Official Position: TV Drama as a Propaganda and Education Tool}

Propaganda and education go hand in hand in China's production of television content. The reason for this is that propaganda is not merely understood in the sense of promoting Communist ideology and improving the image of party and state. This is of course a major aspect of propaganda, and television drama content is ideally meant to reflect the government and party position concerning the Chinese modernization endeavour, thereby facilitating the viewers' confidence in their leadership (15, 97:58 - 99:23). However, and this has become evident from the importance that the government attributes to drama content, television propaganda is more importantly meant to provide "guidance" (daoxiang 导向; I2, 93:14 - 95:40). As mentioned earlier (Section 3.6.ii), television drama is regarded as a medium that supplies viewers with role models. Just like the depiction of questionable morals and values is believed to inspire malicious behaviour in audiences, so is the depiction of positive roles seen as a way of teaching viewers how to behave properly (19, 15:22 - 17:09).

The most explicit form of propaganda in TV entertainment is the main melody propaganda drama (zhuxuanlü dianshiju 主旋律电视剧). The key intention of these dramas is to either glorify the revolutionary history of the party, or promote the image of party and state. Usually, main melody dramas are produced with the financial and institutional backing of the state. A prime example of such a drama is the well-received GAJZ (I13, 23:35 - 27:44), which will be analysed in detail in Section 4.2 of this thesis.

However, political content does not necessarily have to be explicitly propagandistic. Discursive positions also find their way into more commercial drama productions through the multi-layered control mechanisms which are enforced throughout the television drama market. As one interviewee pointed out (17, 42:46 - 46:12), even teen dramas and similar apparently mainly entertaining programmes will have a didactical dimension in China. According to the interviewee, the reason for this is that the government is essentially in 
control of all drama production processes and will always encourage that the ideological requirements of state and party are met (ibid.). The interviewee explained that in such cases the educational content will be embedded in the entertainment aspects of the drama: the famous, beautiful actors and the dazzling set locations are meant to attract viewers, and the values depicted in the drama are meant to teach the audience how to properly lead their lives (ibid).

While both these didactical approaches (the explicit as well as the implicit) construct discursive positions, not all didactical content has to necessarily promote a political message designed by the leadership. For instance, one screenwriter expressed the opinion that television drama should critically depict contemporary social problems. She described how she devised her own scripts with a didactical motivation in mind, but that the educational content presented therein was derived mainly from her own personal experiences with China's drive for modernization (18, 86:17 - 91:52). The same interviewee in fact questioned whether the propagation of the main discourse on modernity (i.e. of content that promotes economic and urban development) was the best didactical approach (ibid.): she argued that the generation of Chinese viewers who grew up during the reform era was already used to modernity and did not require lectures on how to further develop the nation; instead, so the interviewee, television drama should aim to educate people on how to deal with the social pressures that the rapid development in China has created (for example the lack of social security, urban isolation, etc; I will return to this non-official position below). Particularly the target audience for teen dramas, i.e. younger viewers, needed to learn that concerns over a lack of social status or of consumer opportunities did not qualify as a "social problem", and that their commercial aspirations (described aptly by the catch phrase "monthly wage of over 10,000 RMB, car and apartment"; yuexin guo wan, you che you fang 月新过万, 有车有有) needed to be replaced with moral values and ethics (daode 道德; I8, 91:53 - 95:03). 
Whether the purpose of didactical television content is to promote official discourses or other values, the respective television dramas are in either case believed to only achieve their purpose if they fulfil two requirements. First, the discourses and their on-screen presentation are required to be 'close to the people', i.e. reflect the every-day social, cultural and economic reality of the viewers. The idea of 'close-to-life' television content was brought up by numerous interviewees $(11,42: 14$ - 44:34; 12, 97:36 - 99:57; $111,33: 24-34: 40)$, and is also mentioned in SARFT documents (see the quotes from SARFT, 2004, article 19, as well as SARFT, 2006, article 22, provided at the beginning of section 3.6.i). In fact, the 'close-to-life' concept is another trope that is rooted in recent Communist ideology, and that is based on President Hu Jintao's demand that CCP politics should be "close to reality, close to life, and close to the masses" (the so-called 'three closes'; tiejin shiji, tiejin shenghuo, tiejin qunzhong 贴 近实际、贴近生活、贴近群众; Ren, 2004).

The second requirement that has to be met by television drama in order for it to unfold its didactical potential is that its content has to be popular. This brings us to the entertainment function of television drama.

\section{7.il The Alternative Position: TV as an Entertainment Enterprise}

In the official position, the propaganda and educational functions of television drama are linked to television's entertainment functions $(16,05: 38-06: 44)$. The reasoning is that propagandistic and didactical content will only reach viewers if the vessel for that content (i.e. the television drama) appeals to viewers' sense of aesthetics and to their entertainment needs (I1, 42:06 - 43:13). A manifestation of this logic is the already mentioned slogan "unite the three qualities" (i.e. assure ideological quality, artistic quality and viewer appeal). Various interviewees have pointed out this official viewpoint (for instance I4, 96:48 - 97:51; I5, 97:58 - 99:23; 16, 05:38 - 06:44; 113, 56:58 - 59:44), with one director saying: "first you need entertainment, only then can you accomplish that [didactical] function" (xian bixu yule 
caineng caicheng zheyang de zuoyong 先必须娱乐才能裁成这样的作用; 11, 42:06 - 43:13). This

position clearly acknowledges the predominant role that market dynamics and viewers' preferences play in the production and distribution of television content. One producer, for instance, corrected the researcher's assumption that the creative process of producing television dramas was strongly influenced by government regulations and propaganda targets, and instead stated that the main influence were market dynamics. According to the interviewee, production companies had no choice but to create programmes that featured as little explicit political content as possible; he argued that if producers created programmes which explicitly dealt with political issues, "no one would want to watch that" (meiyou ren yao kan 没有人要看; $12,78: 11$ - 78:27).

While the dominant position seems to be that this degree of commercialization is regrettable, and that it decreases the amount of 'healthy' content that is broadcasted (18, 56:42 - 59:41; 19 , 26:21 - 27:50; I11, 74:32 - 75:40), another line of argumentation questions the educational and propaganda function of television altogether. Particularly those involved in privately funded television drama productions argue instead that TV dramas are merely meant to entertain, and should be primarily seen as a business enterprise. For instance, one interviewee voiced the opinion that "television dramas, as well as films and literature, are not schools" (xinosiuuo, dianying, dianshiju dou bushi xuexiao 小说、电影、电枧剧都不是学校) and therefore “do not have the power to solve social problems" (ta meiyou liliang jiejue zhexie wenti 它没有力量解决 这些问题; 112, 59:58 - 60:17). A section from the same interview further demonstrates the commercial function of television dramas (I12, 47:04 - 50:23):

R: Has there been a case in which you felt you wanted to tell a story but it wouldn't sell?

I12: Possibly. Such a case is possible, but if such a case occurs in the television drama industry then I think that's just the way it is. Because in the television drama industry the story is supplied by screenwriters. They are part of the assembly line. I believe that with television dramas, 
their commodity value is more important than their creative value. Drama series are products and not works of art. Because television dramas are meant to be sold. They are not like films, which is why I have always told my students in class... and of course this is just a joke: "I hear they make underground films and art-house films, but I have never heard of such a thing as an underground television drama or an arthouse television drama." (laughs) Because underground and art-house films are types of movies. But there are no underground television dramas and art-house television dramas. Television drama only has one purpose, and that is to be shot, sold to a TV station, and broadcasted. It only has this purpose. So considering this, you have to obey the requirements of the market. I believe that if you... I often tell my students in class: "If you have some story or some message you feel you really need to impart, then you should go and write a novel. But if you are writing a television drama you have to consider two issues: the first is that you need to come up with a product plan; and I believe that the purpose of the product plan is not to please me, but to convince others to buy the product; the best thing is if I like the product and there's also a buyer, that would be the best option. But aside from that, if I actually do not like the product, yet someone buys it... well from the perspective of the product I believe that is still something worth considering. This is the first issue: you have to consider making a product. That's the first issue. So the second issue is that you have to consider that your television drama is meant to be aired on television, and many viewers, including viewers of different age levels, will all watch it. A television drama that is aired during prime time will have viewers of all ages, cultural backgrounds, and educational levels. So if you are broadcasting it at that time, you also absolutely need some sense of responsibility. You will still hope that this television drama will reflect the interests of average people. Maybe that's the one thing to consider. But if you want to express other ideas you should write a film or a novel.

R: Yeah yeah yeah. So we can say that the most important thing is still the market, not political censorship...

I12: It's not about politics. The issue of television drama censorship... it would seem that at the moment this is not a particularly urgent problem, because when it comes to government control, they'll tell you in the beginning: "If you write a homicide drama we will not allow you to broadcast it during prime time." So if at this time I still want to go ahead and shoot the drama, then you already know the result: did they not say "if you write this then we won't let you broadcast it"? It seems that it is very rare that a television drama is not broadcasted because of censorship issues. That seems to be very rare.

According to the above position, television drama is meant to generate profits. In the

interview segment above, this is achieved through the sale of a product to a broadcaster.

However, the commercial 'spill-over' created by product placement in television dramas is also seen as a positive function of this media format. The following is an excerpt from an 
interview with a TV drama director, who was asked what type of television series he would like to produce in the future $(\mathrm{I} 1,47: 04-48: 51)$ :

I1: I think that afterwards I might focus on the things that young people enjoy watching.

R: Why is that?

I1: Well, because I think that this medium of communication is closely linked to the advertisement industry. If I create an interesting television drama, the ratings will be exceptionally high. However, the viewers who watch that drama are older than forty, and their consumer mentality is already set. They are not impulsive. So when they watch they won't go and buy the advertised product. However, if you look at young people, thirty-something and younger, they might see a new type of car in a television drama and they may suddenly think "I want that, after a few years of hard work I will want to buy that car".

Throughout the various interviews, the importance of television drama's commercial

function has repeatedly been emphasised, and one interviewee summed up the debate on the economic dimension of drama production by stating that "the market comes first" (shichang di yi 市场第一; I2, 78:11 - 78:27).

\subsection{Additional Functions: Psychological and Social Relevance of TV Drama}

Aside from the propaganda and educational function of television drama that is so deeply imbedded in the official position, as well as the commercial function attributed to television by entrepreneurs and officials alike, Chinese media experts frequently refer to another function, which is not mentioned in official documents. This is the idea that Chinese audiences need television drama as a form of social release, as a way of compensating for the hardship of life in post-reform China. An example is the popularity of Korean family dramas in the PRC, which are more conservative in their depiction of values than the average Chinese programme $(15,04: 26-06: 15)$. One interviewee explained the reason for this popularity of traditional themes as follows (ibid.):

I5: Well, currently many people in China who watch Korean television dramas will be able to find in them certain things that they themselves 
remember fondly; things that we had in the past, but which we don't have any more. But to be more precise, it's like this: in Korean family dramas, old people are highly respected. Even if what they say is wrong, they are still... <background noise>

R: They have to... <background noise>

15: But they rely on their children, including their daughters-in-law. But what is it currently like in mainland China? Old people's status is not that high in the eyes of their children. I am not saying that they completely lack status, but it is definitely not as high as in Korea. Consequently, when old people in China watch these Korean dramas, they will easily find them appealing. For instance they may derive some kind of satisfaction from them that they cannot get from their own lives. Old people certainly long for respect, and I wouldn't say that currently the young people in China don't respect the elderly, but definitely not as much as Koreans. Also, actually, in most households the relationship between the father and the daughter-in-law is not that good. In traditional Chinese society, for example before 1950, before 1949, many Chinese daughters-in-law did not have much status. They had to obey the elders. However, today, many old people don't have many rights in the eyes of their daughters-in-law, so that is why when they watch Korean dramas they will easily find them appealing.

According to the argument above, Chinese viewers feel that part of their cultural heritage and their traditional (particularly family) values have been lost during the process of modernizing China. Another interviewee makes the same point (I12, 57:05 - 58:25):

I12: Actually, in a society like China's, it has probably gotten better during the last year or two, but in the period before that everyone felt that many personal relations that had been stable in traditional China, very intimate relations... that young people do not have such relations anymore. The family has always been a very important bond in China. However, during the 90 s such family bonds have gradually collapsed. That's because people's desire for material wealth has become stronger and stronger, and material possessions have grown more and more. So the family bonds collapsed, and everyone felt lost. Everyone thought that as soon as they had money, drove a car, and had an apartment, they would be in control of their lives. But since we also got rid of our family sentiments at the same time, we felt saddened. I believe that at that time relatively many dramas were written that depicted such family relations. Relatively many dramas dealt with those themes.

One function of television, then, is to assuage the loss that many viewers feel in light of the perceived corrosion of traditional family values and morals. According to the media experts I interviewed, television dramas fulfil this function in three ways. 
The first of these is to appeal to the nationalist sentiment of viewers. This is particularly true for television dramas that deal with historical aspects of Chinese culture. Such series tend to depict stories from the "golden ages" of Chinese civilization, for example depicting the height of the Han, Tang or Ming dynasties (I5, 92:42 - 97:57). One such example is the series HWDD, which is analyzed in Section 4.1 of this dissertation. The achievements of the Han Emperor Wu (for example his consolidation of Confucian values, his management of ethnic conflicts and his expansion of the Chinese Empire) are viewed by Chinese audiences as inspiring and reassuring $(110,23: 46-25: 01)$. Without the knowledge and pride of their history, Chinese viewers are believed, as one interviewee put it, to suffer "from an empty heart" (xinli shi xu de 心里是虚的; 17, 41:06 - 42:46).

Secondly, television dramas attempt to appeal to audiences' feelings (xingan 心感; 16, 38:21 40:21). They allow viewers to identify with the loss and suffering of the characters, and thereby give them the opportunity to grow emotionally through a cathartic experience. In this sense, television dramas (as well as media outlets such as the internet) have a therapeutically function: they offer psychological and social release, especially for those who have been otherwise marginalised by modern-day Chinese society $(17,72: 15$ - 73:26):

I7: I think television, in fact regardless of whether we're talking about Chinese or foreign audiences, many television programmes evaluate such problems. For example, under conditions such as the current strong trend towards more entertainment and leisure, something arises that... it's not about aesthetics, people are not trying to find beautiful content on television or on the internet, but instead are looking for ugly details. That is to say in China, and you have also spent some time in China, you will come across such women as Furong liejie on the internet, unattractive people like $\mathrm{Mu} \mathrm{Zi} \mathrm{Mei} \mathrm{who} \mathrm{take} \mathrm{all} \mathrm{their} \mathrm{sexual} \mathrm{experiences}$ with men, write them down, and put them on the internet. Or that Furong Jiejie trying to figure out how to deal with her unattractiveness. In the end, many feel that life is exhausting. In our current type of explosive developmental period many don't know who they are and may feel that status is relatively hard to come by. So at such times, because the pressure from society is relatively high, they require some kind of psychological release. 
The third method is closely related to the educational function of television dramas. By depicting everyday social situations, dramas offer viewers hypothetical situations that they can analyze and then base their own behaviour on, or as one interviewee stated (I14, 24:05 24:19):

I14: When it comes to television dramas, the common Chinese people really enjoy analysing the social problems that they themselves grew up to experience.

It is difficult to establish how far these arguments on television's function as a compensator for social problems in China are integrated into the hegemonic discourse. It is likely that the psychological functions of popular entertainment, i.e. its opiate-like effect on the masses, are accepted and possibly even encouraged by officials. The depiction of social problems on television is certainly not prohibited by the authorities, as long as it does not endanger China's stability. However, the dissatisfaction that media experts believe many viewers feel towards the economic and social development of their country, as well as audiences' perceived need to compensate for these inadequacies by either becoming emotionally introspective or turning to historical (i.e. 'feudalistic') traditions, is not likely to be what the Chinese leadership has in mind when it refers to television's purpose of "appealing to audiences" (SARFT, 2006, article 22). In this sense, television drama's social release function should be considered part of the counter-hegemonic discourse.

A similarly unofficial position is the idea that television should facilitate public discussion of everyday problems, thereby promoting pluralism. The basic notion that television dramas provide talking-points for audiences is itself not necessarily counter-hegemonic, and is in some cases associated with the educational function of television (I1, 42:14 - 44:34). However, as one interviewee explained by referring to the American sociologist Daniel Bell, television in China also functions as a place where different conflicting social aspects (economics, politics and culture) are negotiated and coordinated (13, 72:50 - 76:07). In this interpretation, 
television drama is regarded as much more than entertainment; it is viewed as a forum for the negotiation of fringe opinions that can otherwise not be voiced (I3, 76:08 - 77:21). Such an argument implies that the discourses that television disseminates and encourages facilitate pluralism and allow viewers to participate in the construction of China's social and political reality; a position that inherently questions the recurring theme of a passive audience.

\subsection{Summary: The Commercial Interests and Political Targets of Television Entertainment}

The above analysis shows that numerous factors influence the creation of discourses during the production process of Chinese television dramas: the discursive elements of a drama depend on the type of production company that initiates the project (state-run or nongovernmental), on the types of investors involved in the project (from within or from outside the media sector; state-run or privately owned), on the production and marketing strategy of the creative producer, on the work of the screenwriter(s) and ultimately on the director's vision of the final product.

In addition, the interviews have shown that the Chinese television drama market is a highly regulated sector. The creation of discourses is monitored closely by the authorities during all stages of production and distribution. This includes 'hard' censorship tools such as the distribution permits (which are granted only after the detailed content of dramas has been minutely examined by the censorship authorities). However, the conversations with production crew members suggest that the full range and force of the censorship system is rarely applied to curb unwanted discourses and promote politically acceptable content. Instead, a series of 'soft' censorship tools such as production permits or prime-time broadcasting regulations are used; these do not exert direct control over the production process but instead merely provide incentives for the creation of certain content. This generates an environment in which only certain discourses are worked into the drama series: 
screenwriters, producers and directors self-censor the content they produce, knowing that they will only be able to sell and later broadcast products that do not upset the power relations in China's society. In this sense, the political self-censorship is mainly driven by commercial considerations: only content that fulfils broadcasters' aesthetic and technical standards, and appeals to audience viewing habits, will be profitable. An apt summary of the three aspects that define the production of television drama content is provided by the interviewees' much quoted slogan "unification of the three qualities" (san xing tongyi 三性统 $一$ ), the three qualities being: ideology (sixiangxing 思想性), artistic quality (yishuxing 艺术性)， and viewer appeal (guanshangxing 观赏性).

My analysis of media experts' perceptions further shows that, in the eyes of those who are involved in television drama production, television series have a profound influence on viewers, and should consequently function as a social and political tool. The notion that TV content exerts such a strong influence on viewers is overwhelmingly agreed upon by Chinese experts. It is this belief in the media content's potential to affect behaviour which defines the way that television dramas are produced, censored and aired in China. This shows what relevance discourse has: the government's control of the media is supported by a network of concepts, ideas, tropes and metaphors concerning television's social function, its influence on viewers, and its possible harmful effects, which ultimately assure the acceptability of hard as well as soft media controls, and make these controls difficult to contest.

However, the analysis has also shown that the didactical and propaganda functions of television are increasingly augmented (though not truly challenged) by commercial interests. The fact that the television drama market has become more liberalised is substantially influencing the production of content in various ways. The relevant factors include the audience rating system, the television stations' need to make profits from advertisement 
sales, and the government's regulations on production and broadcasting. The feedback that audiences provide concerning television drama content does not actually 'feed back' into the production process in any significant way, but the preconceptions that production crew members and broadcasters have of their target audiences and their viewing habits nonetheless strongly influence the planning, creation and broadcasting of drama content.

\section{Chinese TV Dramas and their Discursive Contents}

After having analysed the factors that lead to the production of discourses in popular Chinese television dramas, this chapter now turns to the main part of this thesis: the visual political analysis of three specific television dramas. Each of the following sections deals with a particular Chinese television drama: the historical drama HWDD is analysed in Section 4.1, the main-melody crime drama GAJZ in Section 4.2, and finally the metropolitan teen drama NCNM in Section 4.3. For each of these television programs, two particular discourse strands are analysed: first, the discourse on governance (focusing on the authorities that govern, as well as the subjects that are governed); second, the discourse on the classical first goal of governance, i.e. security (for GAJZ and NCNM, this means an analysis of public security issues; in the case of HWDD, the analysis focuses on foreign security). Throughout the analysis, additional attention will be given to the question of how government actions are justified, and what role patriotic or nationalist themes play in the depiction of the respective discourse strands. The findings are summarised and evaluated in Section 4.4.

\subsection{Visual Political Discourses in a Historical Drama}

The first series under scrutiny here is the historical television drama HWDD ( $\mathrm{Hu}, 2005)$, which started broadcasting in early January of 2005 and was aired consecutively on CCTV-1 each evening at 20:00 p.m. The 60,000,000 RMB production was filmed by renowned director Hu Mei 胡玫 under the creative production guidance of Han Sanping 韩三评. The fifty-eightepisode epic was produced by the Literature and Art Department of CCTV (Zhongyang 
dianshitai wenyi zhongxin 中央电视台文艺中心), in collaboration with various private investors. The drama is based on a script written by Jiang Qitao 江奇涛, and features a cast of famous actors, most notably TV star Chen Baoguo 陈宝国 in the role of Emperor Wu. The series was promoted as a "realistic historical drama" (lishi zhengju 历史正剧; Zhang, 2005), and ranked $12^{\text {th }}$ most popular television drama of 2005 (Wang, 2006, 249). It tells the story of Liu Che (刘 彻), who later became known as the Han Emperor Wu, and consecutively deals with Liu's childhood, adolescence, his accession to the throne, his military struggle against the Huns (in some of the resources referred to by their Chinese name: Xiongnu 匈奴) as a young and middle-aged emperor, and finally with his latter days as a paranoid and authoritarian old man. The drama includes strong references to imperial rule, as well as foreign security issues, which shall be analysed below. The discursive statements identified in this analysis, as well as the signs used to construct these statements, are summarised in the corresponding tables in Appendix 3.

\subsection{The Humane Ruler}

The discourse strand on governance is featured prominently in HWDD. Due to the drama's focus on imperial rule, governance here primarily refers to governance through state rule. As mentioned earlier (Chapter 1), the drama in fact concentrates so strongly on Emperor $\mathrm{Wu}$ as a personification of the state that it led one Hong Kong journalist to conclude that it was meant as an allegory to present-day politics, commissioned by the Chinese state (Yan, 2005). Although there is no evidence to support the claim that the Han Emperor Wu here serves as a propagandistic personification of the current Chinese leadership (the amount of criticism launched against the drama series by Chinese officials and intellectuals alone makes such a conclusion questionable; $19,19: 43-21: 16)$, however reference to the state and its ruler nonetheless dominate throughout the drama. 
The following visual discourse analysis shall first focus on a sequence from the last episode of the drama, which combines the discourse strand of governance with the discourse strand of being governed (HWDD 58-03). The sequence occurs after the Emperor Wu has lost both his first son, crown prince Liu Ju, and Liu Ju's mother Lady Wei to the power struggles at court. Increasingly paranoid and isolated, the aging emperor starts to doubt his rule and his accomplishments. Before this backdrop, the emperor travels to the countryside with his young son, the new crown-prince Fu Ling, in order to educate him on the achievements of the people there. As the emperor's carriage arrives in the village, the inhabitants gather together in excitement to greet the ruler, lauding him for his success, and inviting him to their homes. The following six central, interrelated discourse fragments can be identified in this sequence:

1. The ruler is close to the people (Figure 15).

Sequence 58-03 shows emperor $\mathrm{Wu}$ as an approachable ruler who is in touch with the masses. This is primarily achieved through the setting of the scene: the mere idea that the supreme ruler of the Han would travel to a small village to personally interact with his subjects is a discursive statement that stands in stark contrast to the proclamation by this drama's producers that the series is a 'realistic' historical representation of the Han dynasty. This fictional encounter in itself therefore functions as a sign, connoting the ruler's proximity to the people. In addition, other signs are used to reinforce this closeness: the use of mise-en-scene (that is: the arrangement of personage and objects in the shots) as well as the language used in the dialogues all point towards an intimacy between the ruler and his subjects: As the emperor mingles with the people, he is set in the midst of a crowd of peasants (shots 28,35 - 38), is shown patting villagers on their backs (shot 37) and is placed in close proximity to a child in the private home of one of his subjects (shots 29-34, which make use of OTS-shots to increase the impression of 
intimacy). The feeling of familiarity is further enhanced by the fact that the emperor and the people converse in colloquial Chinese (as opposed to the formal language used in the court scenes of the drama). In addition, the ritualistic bowing to the ruler that is emphasised in almost all other sequences of the drama (for examples in this last episode, see HWDD 58-10 or 58-12) is handled here as a mere formality, with the emperor urging his subjects to their feet (shot 24). This series of visual and verbal signs can be paraphrased to form the discursive statement:

- The ruler is a man of the people.

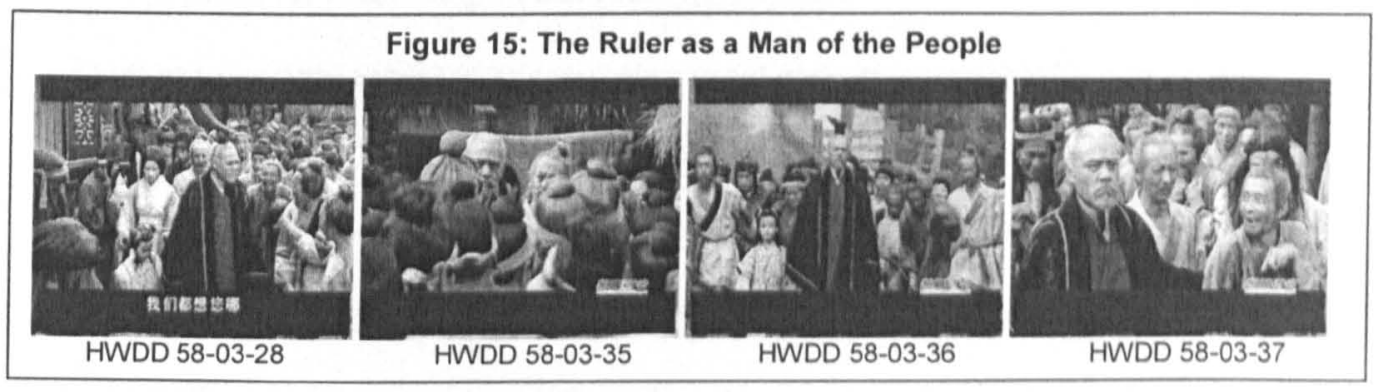

2. The ruler is a fatherly figure to the people (Figure 16).

The personal relation between the ruler and his subjects is further emphasised by various sings that mark the emperor as a paternal figure. First, a combination of mise-en-scène arrangements and medium close-up shots with low camera angels is used to place the emperor physically in a position above his subjects, looking down on them with a kind expression on his face (shots 20, 22 and 26). A verbal sign is then used to further relay the ruler's care and affection for the people: as the emperor leans forward, he asks in a low, mild voice "is everyone alright" (dou hao ba? 都好吧? shot 26). A third sign is then twice used to steer the viewer towards an understanding that the emperor is a fatherly figure: two scenes in this sequence juxtapose the aging ruler with children. First, the emperor visits one of the villager's homes, where he caringly interacts with a crying boy (shots 24 - 32); second, in the final series of shots, the emperor picks up his son Fu Ling and carries 
him in his arms (shots 53 - 58). All of these signs come together to form the discursive statement:

- The ruler cares about his subjects like a father cares for his children.71

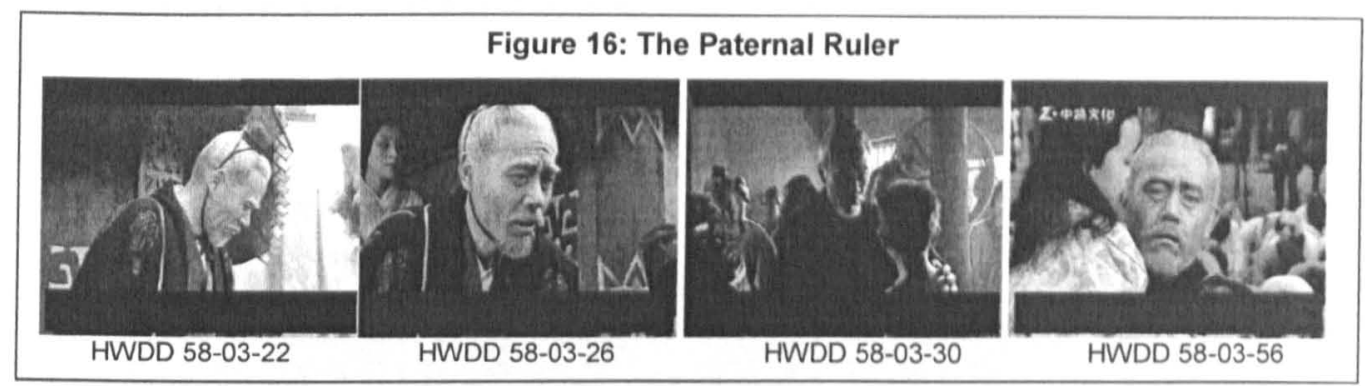

3. The people love and respect their ruler (Figure 17).

Another important aspect of this sequence is the enthusiasm with which the villagers

greet the emperor. The first part of the sequence consists of a juxtaposition of shots

showing on the one hand the arrival of the imperial carriage (shots $02,03,04,06,07,10$,

12 and 13), on the other hand the villagers gathering for that arrival (shots 01, 05, 08, 09

and 11). The coming-together of this smiling, happy peasant crowd is further accentuated by visual signs (a one-armed man waving frantically with his healthy arm; shot 15),

verbal signs (the one-armed man calling "quickly, come look! fellow villagers, the

emperor has come!"; the people cheering "your majesty!"), as well as by an acoustic sign:

the musical score here is a majestic imperial theme, that rises in intensity and reaches its

climax as the emperor emerges from the carriage in shot 16 . Two of the villagers then

directly address the emperor (medium close-up of the men, from high angles), explaining

that they fought for him with valour in the great northern desert battle (one of the men

states that he personally received a medal from the emperor; shots 19 and 20), and very

colloquially praising the emperor as a great and "resolute" man (huangshang, nin ke shi lao

\footnotetext{
${ }^{71}$ The idea of the ruler functioning as a paternal figure is one that is deeply rooted in Chinese traditional thought As an example, consider the Confucian work "The Great Learning" (Daxue 大学), which comments on rule by stating that: "those who love what the people love and hate what the people hate are called the parents of the people" (民之所好好的, 民之所恶恶的, 此之谓民之父母; 10.3). Another example is the work Zhanguo Ce 战国策, which argues that the ruler should "make all men his children" (子元元; 3.2)
} 
han le! 皇上您可是老狠了! shot 21). The villagers then carefully help the emperor down from his carriage (shot 25), and when asked how everyone is doing, they cheerfully proclaim that all is well (shot 27). They go on to tell the emperor how much they have been thinking of him, and then welcome him to their homes (shot 28). In this sense, the sequence states quite unambiguously:

- The people love their emperor.

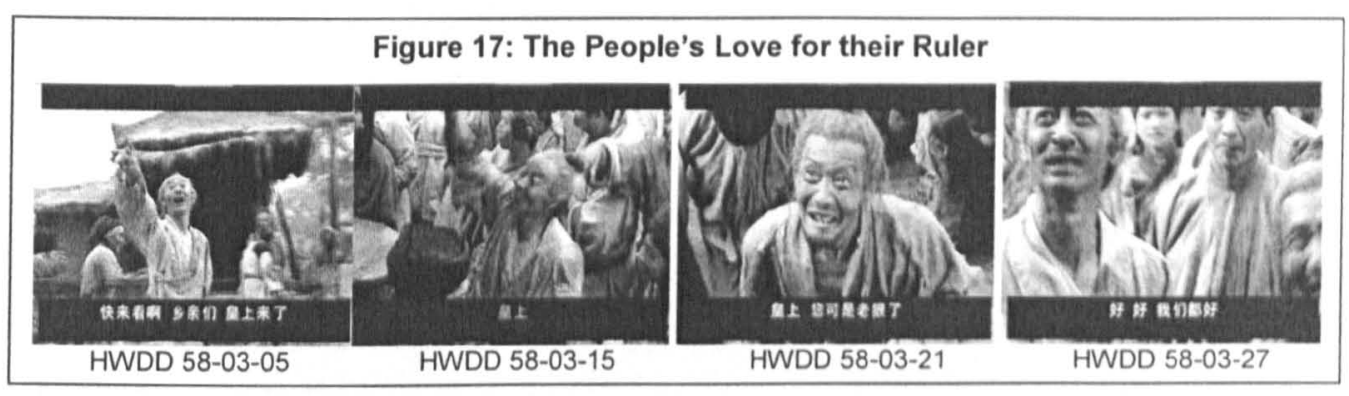

4. The people are suffering (Figure 18).

The next discursive statement concerns the villagers shown in this sequence. The crowd that gathers to greet the emperor is impoverished (dressed in rags) and largely physically disabled: the main village character with whom the emperor interacts is the old man with only one arm; another man has only one leg, yet another walks on crutches, and others wear bandages. The villagers generally consist of women, children, the elderly and the disabled (shots $01,08,09,11,36,37,38$ and 49 ); there is no single young, physically able male among them. The poverty of these people is further emphasised as the emperor visits one of the villager's homes: not only is the room itself bleak and scarcely furnished (featuring only a simple bunk bed and some baskets), but the child that the emperor encounters there is clothed merely in a piece of cloth, and is clutching a feeble piece of corn (shots 29-32). Probably the most significant aspect in this context is the combination of the visual signs of poverty and distress (note the close-up of the boy's pained face in shot 33) with a verbal sign: the child starting to cry. This outcry by the boy is relevant in two ways: first, it stands in contrast to the villagers' verbal assurances to the emperor that 
they are "all alright" (women dou hao! zhen dou hao! 我们都好! 真都好! shots 26 and 27); while the older villagers are trying not to let their suffering show before the emperor, this child cannot help but betray the idyllic scene by crying out in hunger. Second, this moment marks the climax of the sequence: the plot up to this outcry was dominated by the apparent cheerfulness of the villagers; only as the child cries out does the emperor grasp the true extent of his people's suffering (acoustically further underscored by the introduction of a slow, slightly melancholic imperial melody). The remainder of the sequence is then devoted to this theme of hardship. The discursive statement here is:

- The people have suffered severely.

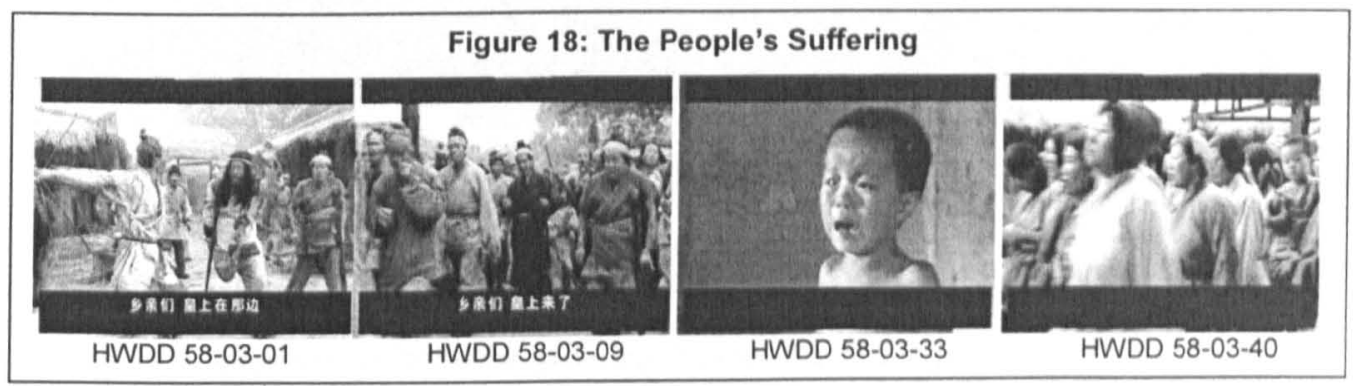

5. The people support their leader's rule (Figure 19).

What is particularly relevant with regard to the villagers' hardship is that the people are not complaining about their suffering, but are in contrast willing, even eager to endure more. After the climax of the sequence in shot 33, the people's cheerfulness declines and a general sullenness descends upon them. As the emperor and his entourage make their way to the desert battle memorial, the people follow their ruler (shots 35 - 40) and drop to their knees as he reaches the monument (shot 42, showing the kneeling people from a high angle and an extremely long distance to give an impression of the crowd's size). A series of shots then follows, showing old crippled men bowing to the emperor, with tears in their eyes (shots 45, 46 and 47). These images, still underscored by the melancholic imperial theme, are linked to the verbal sign of the villagers calling out to the emperor “we want to fight the Xiongnu with you again!" (women hai yao gen nin da Xiongnu! 我们 
还要跟您打匈奴! Shot 46). At the same time, another visual method is used to literally place these men and women behind the emperor: shots 43 and 49, as well shots 51, 52, 55 and 56 (now underscored by the tune of a single melancholic trumpet) are arranged in a way that shows the face of the emperor in close-ups and medium close-ups, with the crowd of villagers forming the background. The discursive statement made here then is:

- Despite their hardship, the people fully support their ruler.

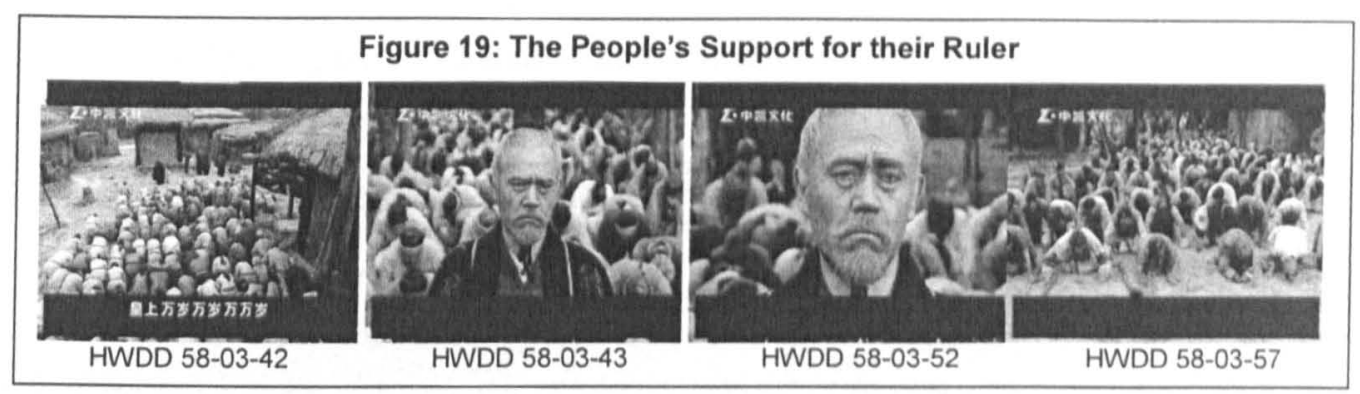

6. The ruler is personally moved by his subjects' suffering (Figure 20).

Finally, the discursive statements on the ruler and his subjects culminate in the emperor's display of affection towards the suffering masses. Starting with the climactic shot 33 , the emperor is shown deeply moved by his subject's hardship and their unwavering support for his rule. Again, various different signs work together to prevent any misinterpretation of this theme: visually, the emperor's anguished face is shown in closeups and medium close-ups throughout the last part of the sequence (shots 34, 39, 43, 49, 51, 52 and 56). Also, the mise-en-scène visually places him before the war memorial in the village (shots $42,48,50,53$, and 58 ) with the suffering people kneeling behind him (shots 43, 49, 51, 52, 55, and 56). Acoustically, these shots are first underscored by the melancholic imperial theme, and then by the highly melodramatic trumpet tune that sets in during shot 50 . Shots 51 and 52 then show the same angle of the emperor's face as he starts to cry, first as a medium close-up, then as a close-up. Two verbal signs are used to set the ruler's crying face into perspective: in shot 51, the emperor responds to his subjects offer to go to war for him again by saying "we can't fight them again" (bu neng 
zai da le 不能再打了); in shot 52 , as the camera moves closer to the ruler's face, someone in the crowd calls out “the emperor is crying" (huangshang ku la! 皇上哭啦! ). To further relay the sentiment of these shots, the emperor's young son Fu Ling is finally brought into the arrangement, telling his father not to cry (fuhuang ni zenme ku le? bie ku! 父皇你怎 么哭了? 别哭! ), and wiping the emperor's tears away (shot 56). The statement made here then is:

- The emperor is a humane ruler who cares strongly about his people.

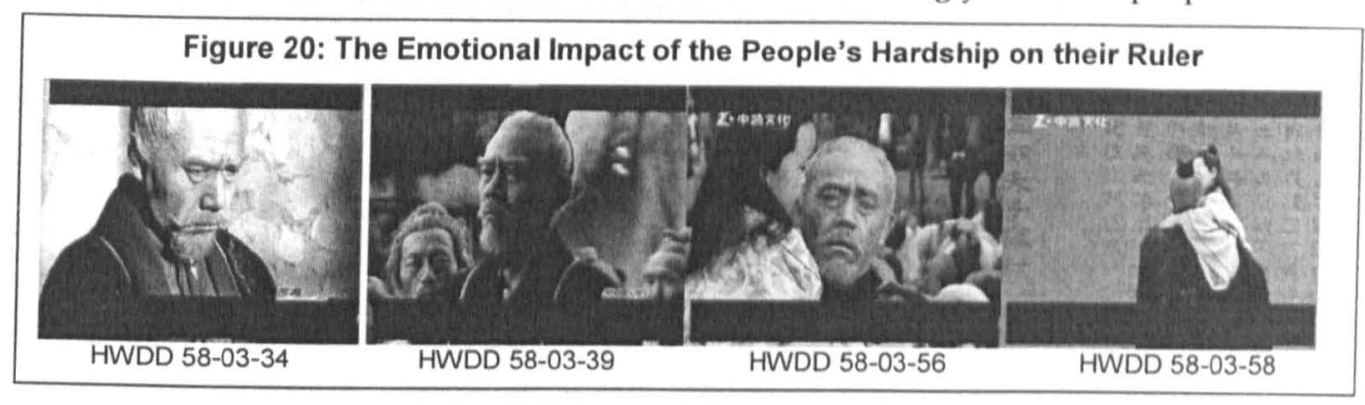

This sequence is unique in its explicit portrayal of the ruler's relationship to the people, but it is by no means an isolated case in HWDD when it comes to the discourse on ruler-subject relations. The discourse fragments identified above, particularly the statement that the ruler is a man of the people who cares strongly about his subjects and their hardship, appear in slightly alternate form in other sequences as well (see HWDD 24-08; 35-02; 38-02; 40-13; 44-10; 55-05; 55-06; 58-07; and 58-08). A good example is HWDD 45-19, which concludes a story arc that deals with Zhang Qian's voyage to the West (this narrative arc is analysed in detail in Section 4.1.ii). In this particular sequence, Zhang Qian returns after thirteen years of hardship and struggle, and is welcomed by the emperor in the great hall of the imperial palace. Again, the emperor is shown as a man who is close to his subjects (in this case to the emissary Zhang Qian and his entourage), cares about their suffering and respects their accomplishments. This is achieved in this particular sequence in a similar fashion as in HWDD 58-03. First, the degree of hardship that the subjects had to endure is emphasised. To this effect, a series of shots is cross-cut, showing on the one hand the battered Zhang Qian 
arriving before the emperor and on the other hand flashback scenes from Zhang's odyssey to the West (shots $33-68$ ). The shots are all extremely short (on average 1.7 seconds per shot), and are linked to each other through a montage of shots with similar camera angles: an extremely long shot at a high angle of Zhang Qian's entourage leaving the imperial capital of Chang' an along a long and dusty road (shot 34) vs. an extremely long shot at a high angle of Zhang Qian arriving in the great hall (shot 35); a medium close-up of Zhang Qian's legs as he falls to his knees in the Western outback (shot 61) vs. a medium close-up of Zhang's legs as he falls to his knees before the emperor (shot 62); a medium close-up of Zhang kneeling in the Western outback (shot 67) vs. a medium close-up of Zhang kneeling before the emperor (shot 68).The increasing shot frequency during the last ten shots of this section (Figure 21) leads up to the instant when Zhang Qian finally kneels, speaking the words "I kneel before the emperor" (Zhang Qian koujian bixia! 张寒吒见陛下! shots 63 - 66).

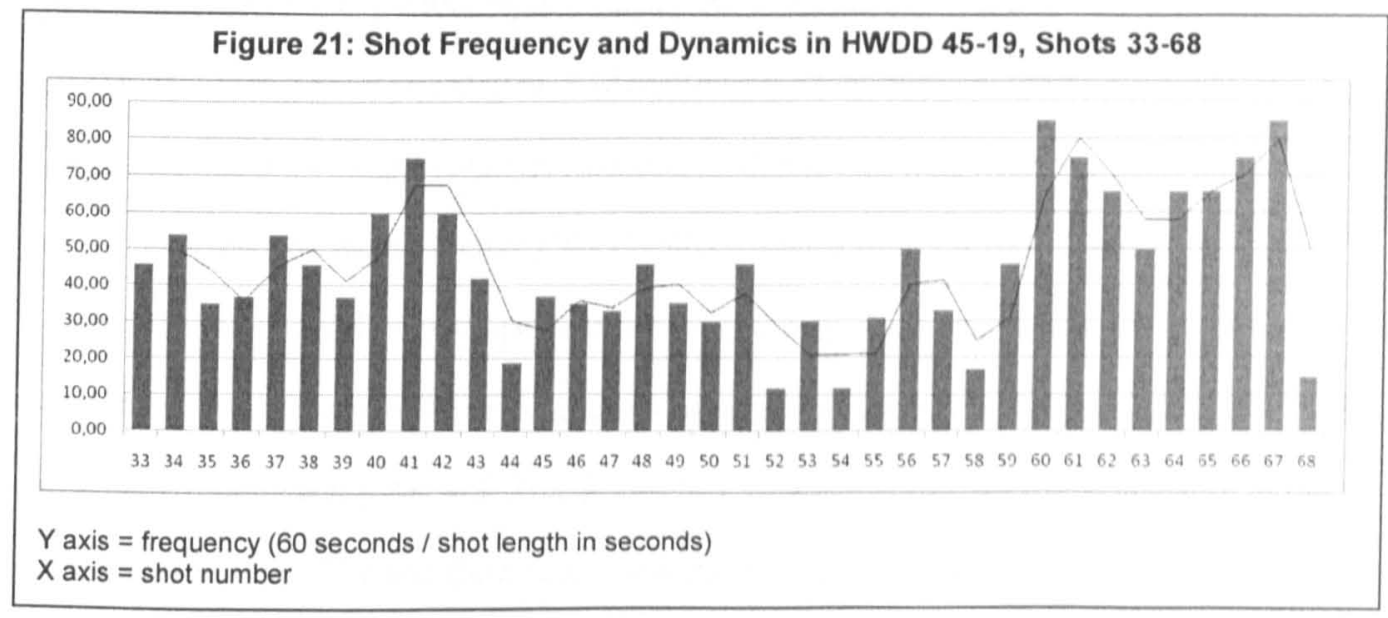

The visual and verbal signs together suggest that the story arc in the West and all the suffering it caused led up to this one moment. Shot frequency, camera angles and montage in this sense form a unit, reinforced by Zhang's words, to make it clear that his hardship was in the service of the ruler (Figure 22). 


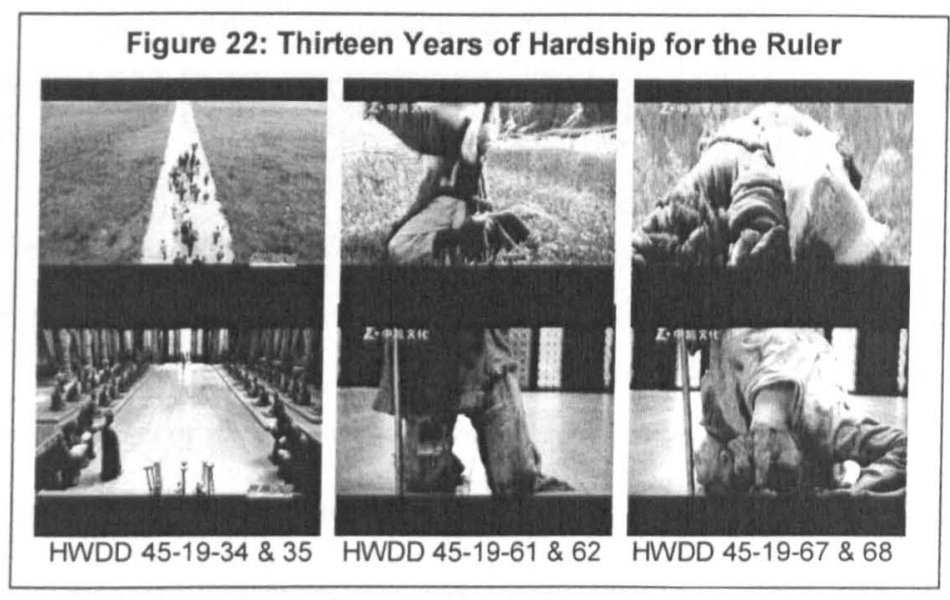

The ruler then rushes down from his thrown (shot 78), embraces the crying Zhang Qian as tears well to his eyes (shots 79-80), and the two men assure one another of how much they have missed each other over the past thirteen years (shots 81 - 90; note the OTS perspective as a method of creating a feeling of intimacy between the two characters). All throughout the sequence, the ministers in the great hall are weeping (explicitly in shots 71 and 91), and melodramatic music is playing (first a dramatic theme that bears a striking resemblance to the theme from the movie Pirates of the Caribbean, then a slow imperial theme, and finally romantic music). Again, the emperor is showing a deep emotional appreciation for his subjects' service (Figure 23), and in order to ensure that the paternal aspect of this affection is not missed, the scene ends with Zhang Qian's young son appearing at the entrance of the hall (shots 92 - 98). The last shots then show the emperor as he picks the little boy up in his arms, while an off-screen narrative voice sums up the importance of Zhang's journey to the West (shots 99 - 102), concluding that the Silk Roads that this journey established "would shine brightly in the historical annals for two thousand years" (hui bing shice liang qian nian de Sichouzhilu 辉炳史册两千年的丝绸之路; shot 102). The sequence ends with the emperor standing at the steps of his hall, saying: "A hero! You are a hero of our great Han dynasty!" (Yingxiong! Ni shi wo da Hanchao de yingxiong! 英雄! 你是我大汉朝的英雄! shot 102). 


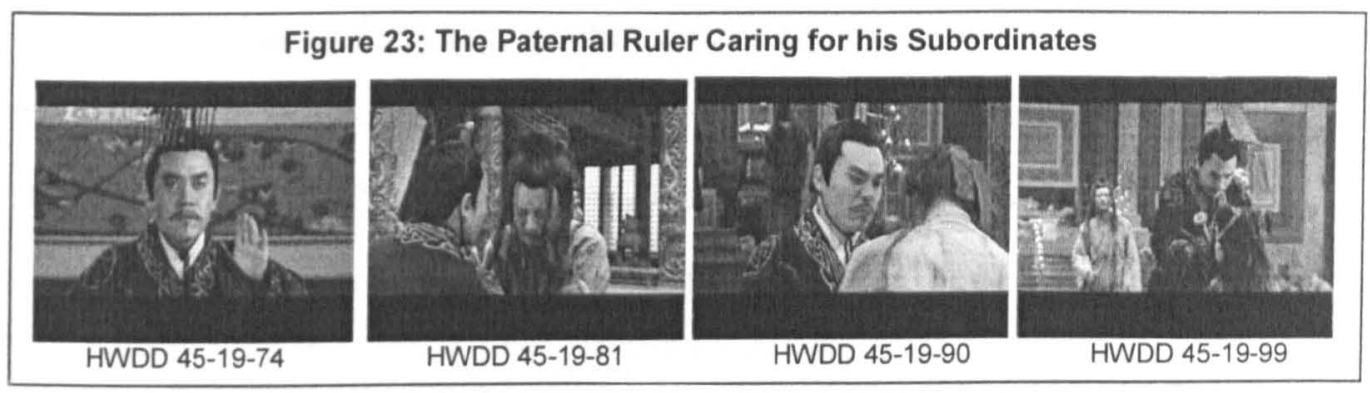

To sum up my analysis of the visual discourse on ruler-subject relations in HWDD, the emperor $\mathrm{Wu}$ is depicted as a passionate humane leader who rules for the people, and for whom his subjects are willing to endure outstanding amounts of suffering. What is remarkable here is that the discourse strand on governing is crossed with the strand on patriotism and national identity; both the sequences analysed here are imbued with an exceptional degree of pathos. I will return to this finding later. First, allow me to turn to the foreign security discourse presented in HWDD.

\section{1.ii Foreign Security and the Establishment of International Relations}

As explained in Section 2.3.v, the state goal of security has two sides: a public security aspect, and a foreign security dimension. The first of these, public security, is not a main feature in HWDD. There are references to the law and to law enforcement, but such themes are usually presented in the context of court intrigues (for example the witch-hunts that consume the Han court towards the end of the drama in HWDD 56-08 \& 09 and HWDD 57-03, or the punishment of officials in HWDD 05-17, 37-06 \& 07; and 46-12 \& 16); they do not stand in relation to society or the nation as a whole, and do not possess a 'public' security dimension as such. The security issue that fundamentally affects the entire state in HWDD is that of foreign relations; arguably the central theme of the drama (my coding procedure identified 85 sequences that dealt with issues of foreign relations, and 279 that dealt with war). For this reason, the following analysis excludes issues of public security, and focuses instead on the security discourse presented in sequences that deal with diplomacy and war. Since themes such as war, peace, the importance of foreign alliances and the threat posed by foreign 
enemies are represented in a large number of sequences, I am analyzing here one particular narrative arc (the planning and execution of Zhang Qian's diplomatic mission to the West; HWDD 26-08/12/17, HWDD 44-01/02/03/07, and HWDD 45-19), as well as one representative sequence that deals with foreign policy (HWDD 54-12).

What is striking is that these sequences present slightly contradictory discursive fragments concerning foreign security issues: On the one hand stands the discursive position that war is destructive and should be avoided, on the other hand the position that war is necessary to counter foreign security threats and to expand the nation. At the Han court, the main foreign security threat, i.e. the war against the Huns, is alternately handled in two different ways. The first of these is an appeasement strategy based on the marriage of royal Han princesses to the leaders of the Huns. Prime examples of how this theme is developed in narrative arcs are the key sequences HWDD 09-14 and 32-04. Such sequences make the discursive statement:

- The Han women being married off to the Huns are kind-hearted and courageous, and they are committing a great patriotic sacrifice for the sake of their nation's safety.

These sequences are not analysed in detail here, but it is worth pointing out how visual signs such as low camera angles and slow-motion shots are reinforced by acoustic signs such as a melodramatic soundtrack to maximise the pathos of these sequences and relay their discursive statement (Figure 24). At the same time, the sacrifices of the Han women and their families are depicted as largely useless against a 'barbaric' aggressor such as the Huns, and the struggle against this northern neighbour is consequently resolved primarily through military action (evidence of this are the elaborate battle sequences of this drama, for example HWDD 01-04, 07-13, 28-06, 32-12, 34-04, 41-19, 43-14, 45-09, 48-11, 51-10, 53-01, among others). Again, the central feature of these sequences is pathos. The mise-en-scène is dominated by vast amounts of soldiers and riders, often edited together digitally to reinforce the sense of 
sheer force. Camera movements across the battlefield (often looking up at riders and soldiers from a low angle, or overlooking the battle from a high angle), slow-motion filming and the ostentatious soundtrack further contribute to the grandeur of these sequences (Figure 24).

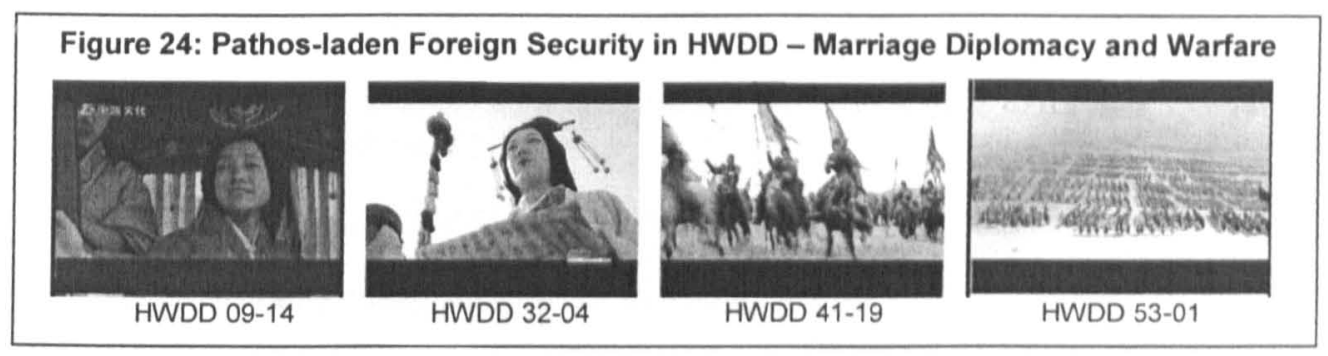

The discussion of whether warfare or marriage diplomacy constitutes the better way of dealing with the Hun security threat occupies much of the drama series. Through the presentation of the appeasement and warfare strategies as well as their outcome, two rival discursive positions are constructed concerning the way in which a foreign aggressor should be treated. A key sequence that pins the two discursive positions against each other is HWDD 54-12. Positioned relatively late in the series, this sequence sums up the respective arguments and presents, both visually and acoustically, the debate's conclusion. The sequence is divided into two scenes: First, the Emperor is shown asking his generals and advisors for their opinion on the foreign security policy (shots $01-97$ ). Then the fate of a scholar is shown who opposed the martial foreign policy of the Emperor and suggested an appeasement strategy (shots 98 - 118).

Let me first turn to the argument for a martial foreign security policy, which is supported in this sequence by the emperor's generals, and is verbally presented by General Li Guangli 李 广利 as follows (shots $04-08$ ):

Li Guangli: You majesty, the Xiongnu's actual strength is already thoroughly weekend. I believe that to resume our marriage diplomacy would make all previous progress null and void. The Han court should continue its punitive expedition against the Xiongnu, should make them pledge their allegiance to us, and have them surrender. 
本广利：潐下，匈奴的实力已经彻底衰弱。卢以为恢夏和亲意味着前功尽 弃。汉庭应对其继续征伐，令其彻底归服，俯首称臣。

When asked what General Li thinks about the opposition's pacifist arguments, he responds (shots 56 and 57):

Li Guangli: I believe these are merely the words of a blind and ignorant scholar.

李广利: 臣以为这简直就是一泿思儒无知的言论。

General Li essentially makes three discursive statements here:

- Peace only gives a weakened enemy the opportunity to regain his strength.

- A weakened enemy should be annihilated.

- The notion of pacifism is for ignorant scholars.

That these statements also represent Emperor Liu Che's views is signified by the Emperor's tone of voice as well as by his gestures (smiling, nodding and leaning back in a satisfied way when the generals are speaking in shots 05,11 and 55, while leaning forward and frowning or dosing off slightly as the crown prince and his supporters are speaking in shots $15,17,33$, $41,53,65$ and 70$)$.

The pacifist argument is more elaborate and is presented by two characters in this scene, the crown prince Liu Ju 刘据 and, more importantly, his advisor Di Shan 狄ll. The core argument is summed up in the following verbal statement by the crown prince (shots 14 18):

Liu Ju: I have always thought: the war against the Xiongnu has already lasted for so many years, and they already abandoned the region north of the Gobi desert; we should cherish this hard-earned peace. I believe we should let the people rest and should cement our relations with the Xiongnu through royal marriage.

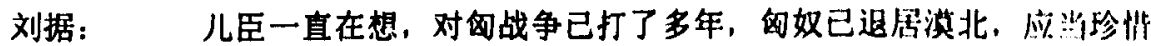

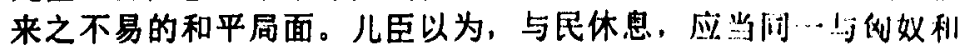
亲。 
The main discursive statement made here is:

- War causes suffering for the people.

Prince Liu Ju is supported in this argument by the scholar Di Shan, who first nervously states that the crown prince's position on marriage diplomacy is correct (shots $21-23$ ), before then stuttering essentially the same statement (shots $38-42$ ):

Di Shan: To resort to arms and fight wars has always been an extremely inauspicious malign matter. It should not, not, not be undertaken lightly. Our dynasty has been at war with the Xiongnu for decades, and the people now long for stability.

狄山：用兵打仗，本来就是一件大不吉利的凶事。不，不不该轻易发动， 我朝与匈奴打了几十年, 现在百姓之心思念安定。

After a failed attempt to impress the emperor with a historical reference to the early, more peaceful Han period (shots 44 - 46), Di Shan continues as follows (shots $50-52$ ):

DiShan: Ever since your majesty dispatched his army on a punitive expedition against the Xiongnu, the riches that our state had saved have been depleted and are almost wiped out. And what is more, the border regions are suffering unspeakable misery. The current marriage diplomacy wouldn't be the same as the one of the past. In the past it was a humiliation (to the Han), but now it is a favour (to the Xiongnu).

狄山：自从陡下兴兵征伐匈奴以来，国内积的财富，已经被消耗㱪尽。 边境地区更是苦不㙋言。现在的和亲和过去已不同了，过去是出等 而现在是恩赐。

Again, the discursive statement that war causes suffering for the people is repeated

(although this statement is an entirely verbal one here; recall the presentation of the impoverished villagers in HWDD 58-03 for a visual and acoustic representation of this discourse fragment). In addition, an economic argument is tied into the discourse, i.e. that wars deplete the state's budget. Finally, the idea of marriage diplomacy is given a new angle through the argument that the general situation has changed since such a strategy was last employed. 
The sequence continues with General Li alienating the scholar Di Shan, and the latter lashing out against the military leader. In this final lengthy section of dialogue, Di Shan further develops the discursive statement that war causes suffering to the common people. In addition, he adds the accusation that the military is not concerned with the average citizen's fate and states that good governance is for the people (shots $58-73$ ):

Di Shan: You, you, I am no doubt a bit blind, but that is only because I am blind with devotion to the emperor. You, you, Li Guangli, where's your intelligence? For what reason are you a general? You have no significant military talent and no significant achievements. You only know how to speak lightly of war, and then take credit for other's achievements during battle. The state has deployed its armies for years and years. The people are suffering heavy casualties. The population size has decreased by almost half, and what is more the Mount Tai region has been troubled by natural disasters this year. The army is a lethal tool and the sage would not use it unless it was necessary. In my humble opinion the current task must be to let the people rest and to implement a benevolent government. How can you not show loving sympathy for the suffering of the common people in the world? How can you ignore it and show no interest? To merely flatter those above like you are doing, general, is nothing else than false loyalty.

狄山：你, 你, 臣固然有些感, 可是臣对皇上是一片思忠。你你李”利又 能聪明到哪去? 你凭什么当将军? 你既无率军之才又无尺寸之功。 只知道轻言开战，以战邀功。国家连年兴兵作战，人民死伤惨重， 户口减少近半，加上天灰今年泰山灰民闹事。兵者凶器圣人不待以 方用之。臣苆以为当今之务，是与民休息，实施仁政。对天下莨生 的痛苦，怎能不体寮关怀? 又怎能罢之不理不闻不问？像将军这样 只知道对上逢迎，那就是不折不扣的伪忠。

The reason I have reproduced Di Shan's dialogue passages in full is that from the verbal statements alone the viewer might receive the impression that the pacifist position includes reasonable arguments. The verbal reactions of the emperor and his generals mark the speaker Di Shan as a nervous academic and may to some degree question the validity of his position (for instance, the emperor stating: "We only see you bragging and spouting empty

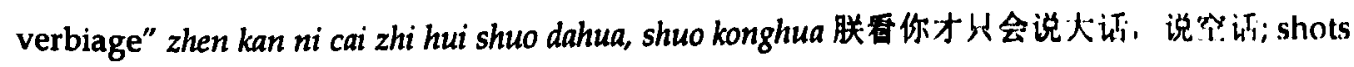
$77-78)$, but at the most the sequence would seem to contrast two equally viable competing 
positions with each other. However, this is not the case. Through various visual and acoustic signs, the statements presented by the crown prince and his advisor are ridiculed, and it is made very clear that the pacifist position is one that only a fool would hold.

First, the crown prince himself is shown as a submissive and weak person, who averts his eyes when speaking with the emperor (shots 14 and 18), who requires the support of an academic to present his argument, and who flinches nervously as he realises that his father is becoming less and less patient with the pacifist arguments of scholar Di Shan (shot 72). The insecurity of Liu Ju is particular emphasised by low camera angels of the crown prince as he is kneeling before the emperor (shots $39,45,54,58,66,72,80,90$ and 97 ; while such low camera angles are often used to signify heroism or glorify characters, the use of this angle here has the crown prince practically crouching down towards the camera, thereby highlighting his submissive and meek character). However, as feeble as the crown prince may appear here, the most important factor that calls into question the pacifist position in this sequence is the presentation of the scholar Di Shan, who is depicted as a thoroughly dislikeable character.

This is achieved first through the make-up and the performance of the actor playing Di Shan: the man is shown with a series of physical defects, such as a nervous twitch of the mouth, extremely bushy eyebrows, beady eyes, badly groomed facial hair, bad teeth and the hint of a cleave in the upper lip. In addition, Di Shan at times speaks with a stutters and generally stands slightly hunched. All in all, this pacifist is shown as a truly deformed figure. The mise-en-scène and the camera operations further stress this fact: When the character of Di Shan is introduced, he is positioned practically crouching behind the crown prince (shots 19 , $21,23,25$ and 27). Also, throughout the entire dialogue with the emperor and his generals, $\mathrm{Di}$ Shan is consistently shown from a low camera angle (all shots of Di Shan from shot 32 to shot 88; again, the angle here gives full emphasis to the hunched posture of the character and 
therefore functions as a pejorative sign). To further assure that the viewer truly grasps that this character is not meant to be taken seriously, an acoustic sign is used to reinforce the various visual signs: Di Shan's speech is in large parts underscored by a comical melody (shots $30-55$ and $78-97$ ).

This comical tune is particularly significant to the conclusion of the scene. After Di Shan has finished his speech, the emperor states with a sarcastic tone that since the scholar has such a favourable opinion of the Huns, he shall receive command of an outpost at the northern border. As would befit a dislikeable character such as Di Shan, the academic starts to haggle with the emperor in order to avoid being transferred to the warzone. After a humorous dialogue between Emperor Liu Che and Di Shan, the ruler gives the scholar a rather insignificant post at the frontier so that he shall prove his worth outside of rhetoric debates. This entire discussion is again underscored by the comical musical theme.

The second scene of the sequence then shows Di Shan's fate: the academic arrives at the border, meekly cowering on the back of a carriage, and is quickly involved in a skirmish with the Huns. The scene is filmed as an action sequence, similar to the ones that reoccur throughout the drama when battles are shown. A high level of camera movement is used to simulate the turmoil of battle, while very short shots are employed to increase the speed of the scene. In general, the dynamics rise throughout the fight, and then sharply drop at the scene's conclusion (Figure 25): Di Shan being slain by a Hun rider. 


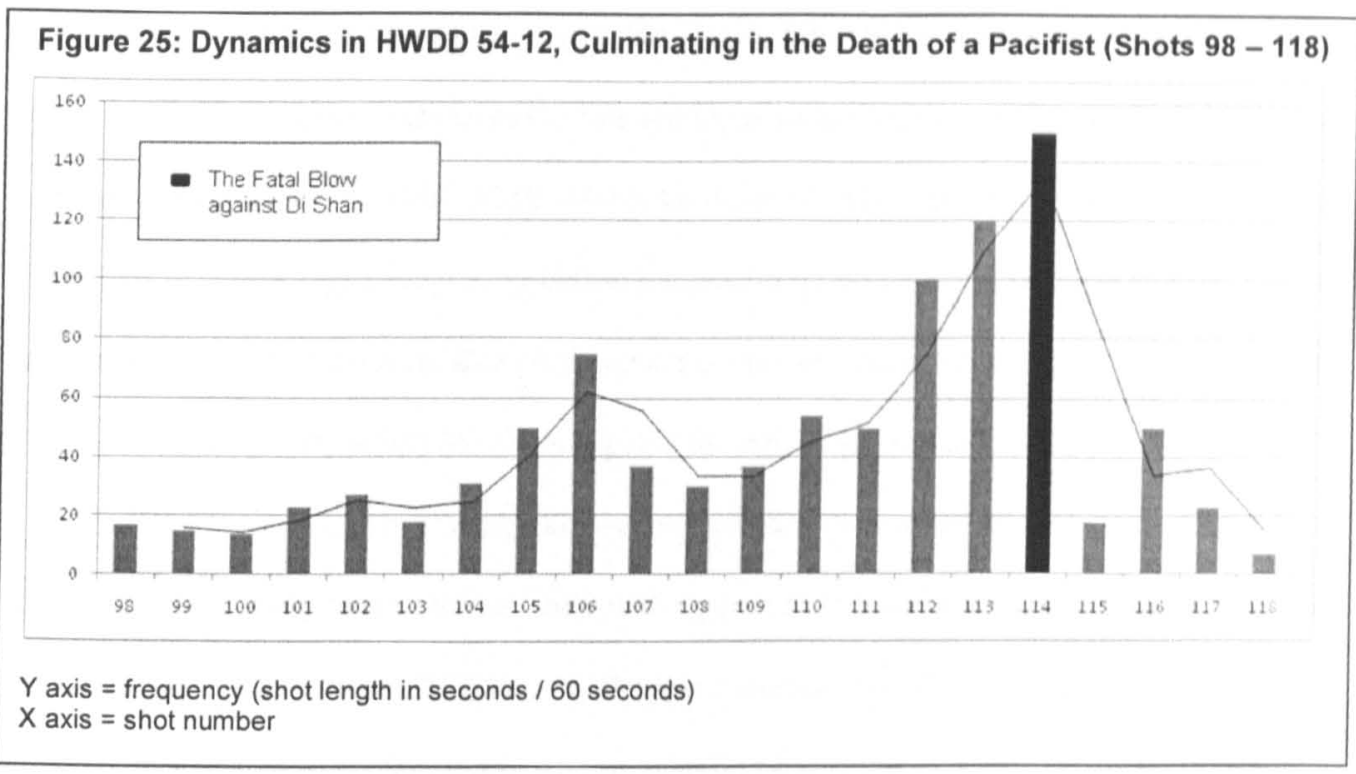

Again, the scene is underscored by the same comical music theme used before. What could otherwise have been interpreted as a tragic or heroic death is then thereby constructed as a satirical event, signifying that the dislikeable pacifist Di Shan found the end that he deserved (Figure 26). In addition, the brutal death of the scholar also functions as an allegory, suggesting that a pacifist sentiment will not hold up against a violent foe such as the Huns. The sequence can therefore be said to make the following two discursive foreign security statements against appeasement strategies:

- Appeasement is a worthless strategy against a foreign aggressor.

- Pacifism is a sentiment that is only shared by the week.

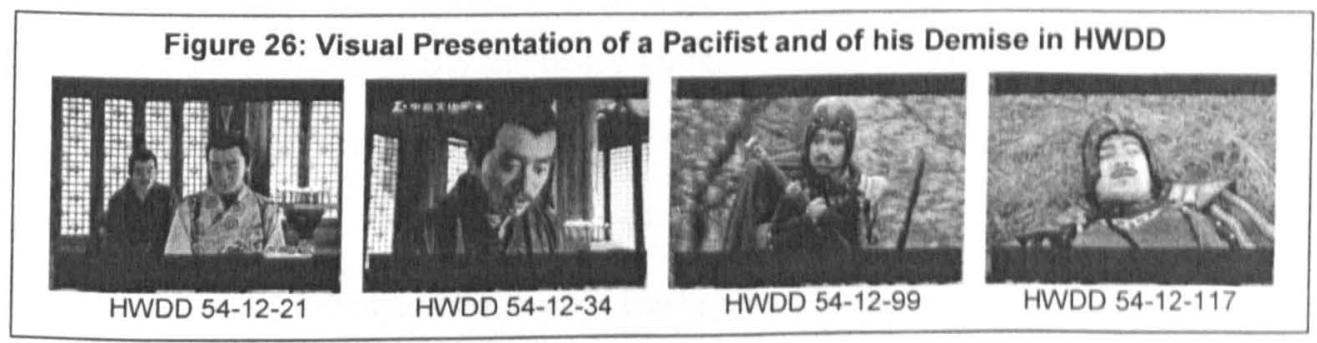


In this sense, the discussions at the Han court clearly favour the martial strategy over a peaceful approach (other examples for this are the discussions on foreign security policy in sequences HWDD 29-15, 30-04, 32-08, 40-16, 43-18, 46-01, 51-22 and 52-01, among others). What is then at first sight surprising is that the discourse on war and peace is treated rather differently in the narrative arc that deals with the Han emissary Zhang Qian's mission to the West. This mission, including its planning and its aftermath, constitutes one of the central storylines in HWDD, and is among the longest narrative arcs of the drama (spanning from episode 28 to 46; only Emperor Liu Che's war against the Huns and various court intrigues involving Princess Liu Ling 刘陵 and the Emperor's uncle Tian Fen 田蚡 are treated as expansively). Throughout this storyline, the martial Han position is first developed and then later reiterated numerous times, but in general a pacifist notion appears to be dominating. But before turning to the pacifist discourse fragments in this narrative arc, let me first discuss what goals are defined for the mission.

The voyage to the West is planned and prepared in a series of sequences in episode 26. First, the emperor receives permission from the empress dowager to send an envoy to the West (HWDD 26-07). He then plans his foreign policy with his advisor Dou Ying 零㫨 (HWDD 2608). When the recruitment notification is posted in town, Zhang Qian takes the announcement off the wall and marches to the palace (HWDD 26-09). He then offers the emperor to take on the commission (HWDD 26-10), and in the following sequence chooses his escort from the ranks of Han's elite soldiers (HWDD 26-11). Finally, the emperor briefs Zhang Qian on his mission (HWDD 26-12). For the purpose of isolating the discursive fragments on foreign security, I have analysed the two central policy discussions in this series of sequences: the conversation between the emperor and his advisor Dou Ying (HWDD 26-08) and the emperor's mission briefing for Zhang Qian (HWDD 26-12). 
The first of these sequences employs a series of relatively lengthy shots (particularly shots 01

- 04) to establish the scene and start the discussion between the emperor and Dou Ying.

During the first two shots, the emperor and his advisor walk from one of the halls to a

pavilion. Dou Ying brings up the topic of the Hun threat, while a dramatic music plays in the background. When the emperor agrees to hear Dou Ying's opinion on the matter, the music fades out and is replaced by bird sounds and a romantic theme (shots 03 and 04). Before this acoustic backdrop, Dou Ying now starts to present his argument (shot 03), and as the emperor and his advisor arrive at the pavilion, a very lengthy low-angle shot (shot 04, with a length of eighty-five seconds) shows Dou Ying standing behind Emperor Liu Che, convincing the emperor of his new diplomatic strategy. This is their dialogue in shots 03 and 04:

Dou Ying: Your highness, the Xiongnu have been threatening our frontier prefectures, repeatedly killing the common people and plundering our properties. Our imperial court should give them a beating! However, your highness should first try to seek for peace through royal marriage since you are just in power. I believe that in the long run the court requires its own strategy for stopping the Xiongnu.

Liu Che: We have long since wanted to put them in their place. What sound strategy does our minister have?

Dou Ying: Most recently, the Xiongnu prince Ah Hur surrendered his troops. He said that the Xiongnu had already beaten the king of the Indo-Scyths. They cut off the king's head and used his skull as a drinking vessel. Even though the Indo-Scyths fled far away, they nonetheless hate the Xiongnu bitterly and have been continuously waiting for an opportunity to seek revenge. Only they did not have an ally who would help them beat the Xiongnu. I believe that if we dispatch an envoy, contact the Indo-Scyths, and arrange to beat the Xiongnu together, then controlling the Xiongnu in the future will not be hard.

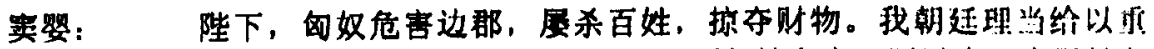
击! 但陆下初登大位, 我想还是必须坚持和亲。居以为, 为了长久 之计，朝廷必须有一套战略来制止匈奴。

刘彻：朕早晚要收拾他们！旅有何良策? 
实赠：前不久，匈奴小王阿胡儿，束部归降。他说匈奴已经打收了月氏 王。他们把月氏王的头颅割下来, 制成了酒器。虽然, 月氏人逃 到了很远，但是月氏国痛恨匈奴人，一直寻机报仇。只是苦于没 有盟邦, 帮助他们, 共出匈奴。臣以为, 若我们能派出使臣，与 月氏人联络，约其共击匈奴，这样，未来制服匈奴就不难了！

A number of relevant discursive statements are made here verbally:

- Foreign security policy should be concerned with the safety of the common people (note how the suffering of the common people spins both ways, here supporting the position that warfare is the best solution to a foreign security threat).

- The Han have the right and the obligation to punish foreign evil-doers.

- Appeasement can only be a temporary solution; in the long run, foreign security threats need to be answered with military force.

- The Huns are violent and uncivilised barbarians.

- Other Asian nations also despise the Huns, and since the enemy of my enemy is my ally, an alliance with such an ally can help win the war.

The rest of this sequence then has the emperor order Dou Ying to launch the new diplomatic efforts by drafting a recruitment statement, and to simultaneously continue the appeasement strategy towards the Huns. As the two men discuss what qualities an envoy to the West would have to possess (courage, insight, a firm and tenacious disposition, health, strength and loyalty), the shots of the emperor now show him from a low camera angle. To combine these verbal statements with this choice of angle, with the romantic music as well as with the general mise-en-scene (servants standing behind the emperor, bowing; also the positioning of the character Dou Ying, i.e. either standing behind the emperor or bowing to him from the lower right-hand side of the frame) not only contribute to the sense of majestic grandeur but also functions as a premonition for the heroic themes that will later strongly dominate this narrative arc. Finally, an acoustic sign is used to complete the emperor's policy choices: as Dou Ying bows and confirms the emperor's orders, the sound of a gong concludes the sequence (shot 13). 
The discursive fragments on foreign security in HWDD 26-08 are then repeated and further developed in HWDD 26-12, as the emperor informs Zhang Qian of his orders. Even though my intention is not to criticise the film-making methods adopted to create the dramas analysed here, it should nonetheless be pointed out that this particular sequence is an example of faulty cinematography and editing. The sequence is arranged as a standard dialogue scene, with the two characters sitting across from one-another at a slight angle. After an extremely long high-angle shot that shows the general setting, the line-of-sight between the emperor and Zhang Qian is established in shot 2 (main shot), and the conversation is then largely shown through juxtaposed OTS shots. There is nothing wrong with the sequence up to this point, except maybe for the rather low level of lighting. However, the camera then repeatedly moves across the line-of-sight, and images from various contradictory angles are edited together (for instance shots 07 vs. 08,11 vs. 12, and the entire section 16 through 23), giving the sequence a rather confused appearance. Such a break with conventional film-making techniques could of course be intentional, in which case it would be meant as a specific visual sign (such an approach could, for example, be conceivable for certain avant-garde or art films). However, my analysis of this sequence was not able to identify any content-related reason for adopting such a visual sign, and my conclusion is that whichever director or assistant director was in charge of filming this sequence was either not aware of the standard convention on dialogue filming which apply to all other scenes of the drama, or thought that the cinematographic rule of never crossing the line-of-sight could be ignored here.

Be this as it may, my analysis was still able to isolate four visual signs that function despite the otherwise slightly corrupted arrangement of shots: 
1. The dominant use of OTS shots (even at contradictory angles) suggests an intimacy between the emperor and his subject, Zhang Qian. In this sense the camera angle reinforces what the viewer has already learned earlier in the drama (that the two men are childhood friends) and what is being stated verbally during the first part of the conversation (that they share childhood memories of the earlier diplomatic strategies towards the Huns; shots $02-07)$.

2. Similarly to the sequence discussed above, the emperor is again shown from low angles (shots $08,11,14,17,19$ and 21). These shots all show the emperor's profile and are backlit with candles, shrouding the emperor's face in a magnanimous glow.

3. Both during the first and the last arrangements of shots, one particular perspective of the emperor is slowly zoomed in over an extended series of shots. The first case is the series of shots $02,04,06$ and 13 which leads to the medium close-up of the emperor used in shots 16 and 23. It spans across the first part of the dialogue, in which the emperor and Zhang Qian recapitulate the past, and gives emphasis to the emperor's memories of his sister being married off to the Huns for diplomatic reasons. Similarly, the second series of zooms consists of the four shots $24,26,28$ and 30 , which again lead to a medium close-up of the emperor (shot 33). In this case, the shots tie together a piece of dialogue in which the emperor explains that he is placing his trust in Zhang Qian, concluding with the image of the pleased emperor after Zhang Qian has vowed his loyalty to the Han cause.

4. Three flashback-shots are used to dramatize the verbal account of the emperor's sister being sent off to the Huns (shots 09,10 and 15, which are taken from sequence HWDD 09-14). 
In addition, two acoustic signs are relevant: First, the persistent sound of a watermill working in the background; this gives the sequence a tranquil feel and reinforces the intimate nature of the conversation between the emperor and his subject (who are having their military briefing not in a strategy room at the palace, but at a lush scenic spot in a garden at night). Second, as the flashbacks set in, a melodramatic music theme starts to play; this further underscores the emotional impact that the memories of his sister have on Emperor Liu Che. What is then stressed here through visual and acoustic signs is that:

1. The emperor and Zhang Qian have an intimate and friendly relationship;

2. The emperor is a magnificent figure;

3. Zhang Qian is loyally devoted to the emperor;

4. The emperor suffers from the memories of his sister having been forcefully married to one of the Huns.

These sentiments provide the frame for the following lengthy speech by the emperor:

Liu Che: The most profound memory of our life was when we saw our older sister Princess Nangong off as she left to be married to the Xiongnu. From that moment on we understood what suffering was. While the Xiongnu were clearly burning and killing our soldiers and civilians, and looting the frontier regions, our father was worried about the domestic situation. In order to avoid a war, he not only avoided dispatching troops to resist this humiliation, but instead married his biological daughter off to a far-away place. On the night before her departure, our older sister was crying as she weaved a string of chimes. We asked our sister why she was doing that, and she said: 'If our father has a long life, our family will have the chance to be reunited one day.' She was truly pure! She couldn't know the hardship of human life.

We can never again tolerate that the safety or danger of our nation be placed on the shoulders of women. Appeasement through marriage... we have always wanted to one day change all of that! Change all of that. From now on diplomatic efforts shall be equally important to military affairs.

Zhang Qian, we place our hopes in you! The rise and fall of the great $H a n$ and the safety or danger of our nation rests with your diplomatic mission to the western regions. We originally wanted 
to present you with a map of the western regions from our secret archives at Lingtai but in the end we could not find one. It would seem that the exploration of this poor and desolate route depends entirely on you. Out there you shall raise the national prestige of the great Han and shall behave in the manner which can present the magnificence of this great nation. Do not let the northern tribes look down on us!

刘彻：朕这一辈子最为刻骨铭心的一件事情，就是送姐姐南宫公主远嫁匈 奴。从那时候开始, 朕才知道了什么叫痛苦! 明明是匈奴在边境烧 杀痛掠我军民，父王顾忌国内形势，为避免战争，不但不起兵抗 侮：反将亲女儿送出远嫁！临走的前天晚上, 姐姐一边哭着, 编著 风铃带。朕就问姐姐，你为什么要这么做? 姐姐说: 若父王还能 长寿, 我们一家人就还会有相见的一天。姐姐太单纯了! 她就不能 体会人生的艰难!

朕再也不能容忍将国家的安危放在女人的胸脯上! 什么和亲一一朕 早晚有一天一定要改变这一切! 改变这一切。就从现在做起! 除了 军事准备之外, 外交努力, 同样重要。

张寒, 朕寄希望于你！你此次出使西城，事关我大汉兴亡，社稞安 危。朕本想在灵台秘库中找件西城的地图给你，但竟找不到一件。 看来这破荒之路只有菲你去探了! 你在外面要昭扬我大汉因威，凡 事要有大国气度。不要让胡人小看了!

The first paragraph expresses the emperor's pain about the loss of his sister and again makes the discursive statement mentioned above: that the women being married off to the Huns are kind-hearted and courageous, and that they are committing a great patriotic sacrifice for the sake of their nation's safety. The last paragraph clarifies the importance of Zhang's mission by making the following discursive statements:

- Opening-up the West is an extremely dangerous undertaking.

- Opening-up the West will contribute to the greatness of the Han nation.

What is particularly important is the second paragraph. Here, the emperor first states that from that point on the appeasement strategy towards the Huns (i.e. marrying royal women to Hun leaders) shall stop. Note how the foreign security discourse is here crossed with the gender discourse: the emperor suggests that his father was weak and indecisive in his foreign policy, so that women had to make patriotic sacrifices for the nation's safety; now, in order to spare the Han women, it is time for the Han men to take action and organise their 
military opposition against the Huns. In this sense, the appeasement notion is giving a feminine touch, opposed to the male connotation that military action receives. The emperor then continues in his speech, stating that diplomacy shall now be viewed as similarly important as military activity. This would normally be a contradiction, seeing as the previous appeasement strategy was a form of diplomacy as well. However, the term "diplomacy" (waijiao 外交) is here redefined to signify alliances with other foreign nations in order to wage war against the Huns, while appeasement strategies are no longer categorised as diplomatic measures. In this sense, this sequence lays the groundwork for the martial foreign security strategy that is then presented and further developed throughout the rest of the drama.

Zhang Qian's actual mission to the West and its discursive content shall be exemplified by four sequences in episode 44. First, in sequence HWDD 44-01, Zhang and his battered entourage arrive in the Central-Asian state of Fergana (Dayuan Guo 大宛国) and are astounded by the wonders that the bustling market streets hold in store. Second, Zhang Qian speaks to the minister of Fergana; he establishing trade relations between the two nations, is offered help in finding the Indo-Scyth nation and finally makes his way to the Indo-Scyths' border (HWDD 44-02). Third, Zhang has an audience with the queen of the Indo-Scyths during which he presents the Han cause (HWDD 44-03). Finally, he attends a meeting of the Indo-Scyth elders, during which the foreign policy towards the Han empire and the Huns is decided (HWDD 44-07).

The dominant foreign relations theme throughout these sequences is not so much war, but much rather trade, as is amply demonstrated upon Zhang's arrival in Fergana: the CentralAsian region is depicted in the sequence HWDD 44-01 as an exotic place with an abundance of valuable commodities. Allow me to summarise the signs used to construct the tradenation Fergana: 
1. Acoustic sings: shots 01 through 33 are underscored by a lively electronic (!) oriental theme and feature constant shouting as well as the sounds of animals (camels and horses).

2. Visual signs: the sequence is characterised by a high degree of camera movements (shots $01-04,06-07,11-14,16-17,19,21-23,26-29,31-33$ and $37-41$ ), in at least one case achieved by using a crane (shot 02 ). High angles are repeatedly used to establish a sense of the surroundings (shots 02, 11, 18, 30 and 34). Dynamically speaking, the average shot-length of the sequence is 4.6 seconds, but numerous series of shots are nevertheless much more fast-paced (for instance shots $12-15$ and $22-32$, which are all significantly shorter than four seconds; the high average shot-length is mostly due to a few exceptionally lengthy shots, such as 02,21 and 33). Finally, the mise-en-scène repeatedly places exotic animals and goods in the frame (shots 01,02 , $04,07-10,12-16,18-23,24,25-30,31$ and $32-33)$, often captured by the camera in close-ups.

3. Verbal signs: Throughout the sequence, Zhang Qian makes very colloquial statements to his friend Gan Fu and to his family members such as: "Look over there!"(kan nabian 看那边; shot 02), "How lively! This isn't bad!" (duo renno ah! zhe ge bu cuo ah!多热闹啊！这个不错啊！shot 03), “Look at this!” (ni kan zhe ge 你看这个; shot 07), and "Go over there. Look, what is that they are selling?" (ni qu nabian, kankam na mai de shi shenme? 你去那边，看看那卖的是设么? shot 11). In addition，Zhang repeatedly asks the local merchants what they are offering ("what is this", zhe shi shenme? 这是什么? shots 8 and 11, and in slightly altered form in shot 19). He and his entourage then express their appreciation for the exotic foods ("It's really fragrant!" zhen xiang ah! 真香啊! shot 7; or "sweet", tiande 甜的; shot 10), or laud the beauty of a

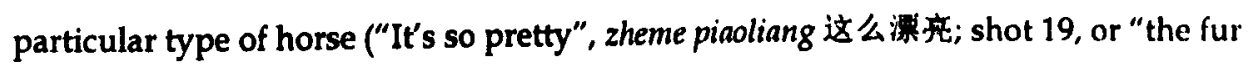
is really nice", zhe mao zhen haokan 这毛真好看; shot 28). In addition, the local 
merchants respond with friendly statements, offering Zhang and his entourage to try their food ("Try them!" changchang! 尝尝! shot 12), and even giving away samples for free (“Take it. You don't have to pay." nazou ba! bu yao qian! 拿走吧! 不要钱! shot 17).

All in all, the sequence makes the following discursive statements (Figure 27):

- The Central-Asian state of Fergana is a bustling and exotic nation.

- The Central-Asian people are merchants who sell exotic goods.

- The Central-Asian people are hospitable and friendly.

- The Han envoy and his entourage are curious and willing to try exotic new things.

Figure 27: The Exotic Commodities of Han's Central Asian Neighbours (HWDD 44-01)

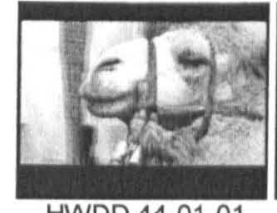

HWDD 44-01-01

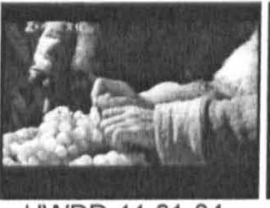

HWDD 44-01-04

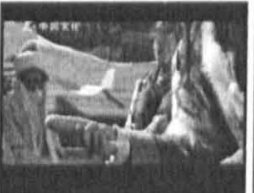

HWDD 44-01-08

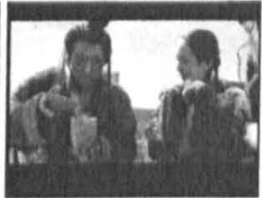

HWDD 44-01-14

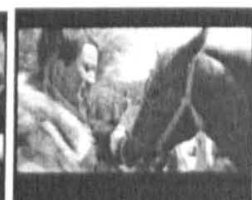

HWDD 44-01-33

These discourse fragments essentially justify the diplomatic effort that is to follow. Towards the end of the sequence, the Han envoy and his people are stopped and questioned by Fergana soldiers; at this point, Zhang Qian produces the seal of the emperor and demands to see the king of Fergana (shot 37; this shot establishes the authority of the Han envoy verbally, acoustically through a suspenseful musical theme and visually through the pronounced position of the red seal in the frame). Zhang's encounter with the king is not shown in the drama series, but the next sequence shows that meeting's conclusion (HWDD 44-02).

In this next sequence, Zhang has a conversation with the minister of Fergana, asking him for help in finding the Indo-Scyths. At the same time, the two men talk about the Han dynasty's silk products and about the precious Hanxue horses (hanxue ma 汗血马) that are bred in Fergana. Before Zhang rides on to his next destination, the two men agree to establish 
amiable trade relations (Figure 28). Their conversation is set in the exotic streets of Fergana's capital (note the establishing shot of the market, with a camel in the frame; shot 03) and is given a tranquil touch by the romantic music theme, by slow camera movements and by at times lengthy shots (particularly at the beginning, with shots 02,04 and 06 each being longer than 10 seconds). The relaxed and friendly atmosphere is further emphasised by images of Zhang Qian's little son playing with a toy drum (shot 16; note also the continuation of the drum sound off-screen in shot 17), and by the demeanour of Zhang and the minister, both of who are smiling or laughing throughout their interaction (see in particular the concluding shots of the scene, shots 21 and 22). Also, the conversation between Zhang and the minister consists in large parts of OTS shots, suggesting an intimacy between the two characters (shots $07-10,12-15$ and $17-22$, OTS perspectives therefore making up sixty per cent of the conversation). The section of the dialogue between Zhang and the minister that deals with trade goes as follows (shots $04-07$ ):

Minister: Ferghana is a land of grapes, alfalfa, and precious horses. Ferghana's merchants would very much like to sell these things to the Han dynasty and trade with you, bringing back in exchange that beautiful silk.

Zhang Qian: Alright! When I return to the Han, I will certainly report your king's wishes to our emperor. I believe that our emperor will definitely also wish to trade with your honourable state.

Minister: I hope that day will soon come!

国相: 我大宛是蔽萄之乡, 苜借之国，宝马之邦。大宛的商队十分愿意把 这些东西贩运到汉朝, 与你们通商, 换回那美网的丝绸。

张塞：好！本使若回到汉朝，一定把你们国王的原意向我们的皇帝报。 我想我们的皇帝也一定非常岩意跟费国通商啊!

国相: 希望这一天早日到来！ 
These verbal statements tie into what has already been established in the previous sequence: i.e. that the Central-Asian people are eager to trade their exotic goods. Considering the discursive statements made in that sequence, this section now adds the discourse fragment:

- Trade is a mutually beneficial exchange of rare goods between friendly societies.

Figure 28: Establishing Trade Relations in an Exotic yet Friendly Surrounding (HWDD 44-02)

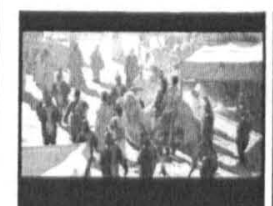

HWDD 44-02-03

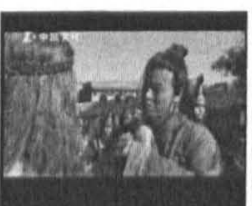

HWDD 44-02-15

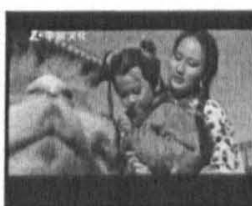

HWDD 44-02-16

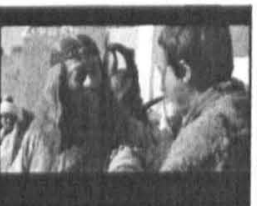

HWDD 44-02-21

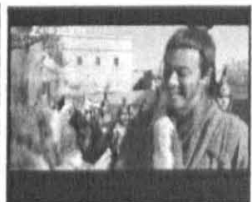

HWDD 44-02-22

The rest of the following dialogue has two functions: first to establish how precious the Fergana horses truly are, and then to prepare Zhang Qian for his visit to the nation of the Indo-Scyths. Here, the minister is given a line of dialogue which rather suddenly moves the focus away from trade-related issues. When asked by Zhang where he would be able to find the Indo-Scyths, the minister replies:

Minister: West of the ridge of Asia, on the northern banks of the river Gui. The great Indo-Scyths are a great nation with a population of over one million people and two hundred thousand crack troops.

国相: 在忽岭西, 协水北岸。大月氏乃是一个大国, 人口一百多万, 精兵 二十万。

Considering that the minister was not asked about the size of the Indo-Scyth state, and certainly not about their military strength, the second sentence in this reply may seem slightly out of place here. It is, however, a logical (though possibly awkwardly placed) verbal statement: the viewer is reminded that despite the discourse on amiable trade that has been developed in the scenes since Zhang's arrival in the West, the main purpose of the mission is to recruit military allies for the war against the Huns. It is with this information in mind that we now witness how Zhang Qian leaves Fergana, travels to the borders of the Indo-Scyth 
nation (shown in the second scene of this sequence; shots $24-51$ ), and finally arrives before the queen of the Indo-Scyths to present his commission (HWDD 44-03).

This next sequence then essentially presents the two frequently reiterated discursive positions on war and peace, i.e. the statement that war is strenuous for the people (here taken up by the queen) vs. the statement that a barbaric enemy such as the Huns can only be dealt with through military force (here supported by Zhang Qian). The discussion between Zhang and the queen of the Indo-Scyths (and hence the conflict between these two discursive positions) escalates in shots $25-42$, and culminates in Zhang convincing the queen of his argument. This is the respective section of dialogue:

Queen: What I am trying to tell you is that the Indo-Scyth people no longer wish to see any warfare! How glorious is peace time! The great Indo-Scyth people would rather forget the painful past.

Zhang Qian: I am shocked by what your majesty is saying! What about that year when the Xiongnu acted in bad faith and launched a surprise attack on you forcing 700,000 of the Indo-Scyth people to migrate westward from the Qilian mountain range. 200,000 people were buried under the vast sands of the deserts and prairies. When you reached the northern mountains, (the Hun leader) Yi Zhixie cought up with you. It was that battle during which the former king of your noble nation was murdered by the Xiongnu. The Xiongnu were savage to the utmost, using the king's skull as a drinking vessel.

Queen: Please say no more!

月氏女王：但我想告诉汉使的是，月氏人已经不想看见战争了！和平的时代是 多么的美好! 大月氏人宁恳忘记痛苦的过去。

张寒：女王所说，令本使十分震惊！想当年匈奴人不顾信义, 对你们发动 突然䓯击, 道使七十万月氏人从祁连山举族西迁。途中二1·万人被 埋在茫茫的大漠和草原。你们在北山落了脚, 伊稚斜追到了北山。 贵国的前国王就是在那次战斗中被匈奴人杀害的。匈奴人凶残至 极，将国王的头顾做成了酒林。

月氏女王: 请你不要再说！

Throughout this section of dialogue, the shot frequency continuously increases (Figure 29). So, also, does the dramatic musical score, which undergoes a crescendo towards the end of 
this section. Both signs emphasise what Zhang Qian is expressing verbally: that the Huns are savages. An additional visual sign is used to signify that Zhang Qian's words are indeed conjuring up the queen's memory of past defeats at the hands of the Huns: shots 32 and 34 show Hun riders storming across the prairie. The symmetry of the shot arrangement reflects the impact that Zhang's words have: shot 31 shows Zhang, shot 32 the Hun riders (presumably attacking the Indo-Scyths), shot 33 shows the queen contemplating Zhang's words, shot 34 again shows Hun riders, and shot 35 has Zhang Qian continuing his speech (Figure 30).

Figure 29: Dynamics of Zhang Qian Convincing the Queen in HWDD 44-03 (Shots 25 - 42)

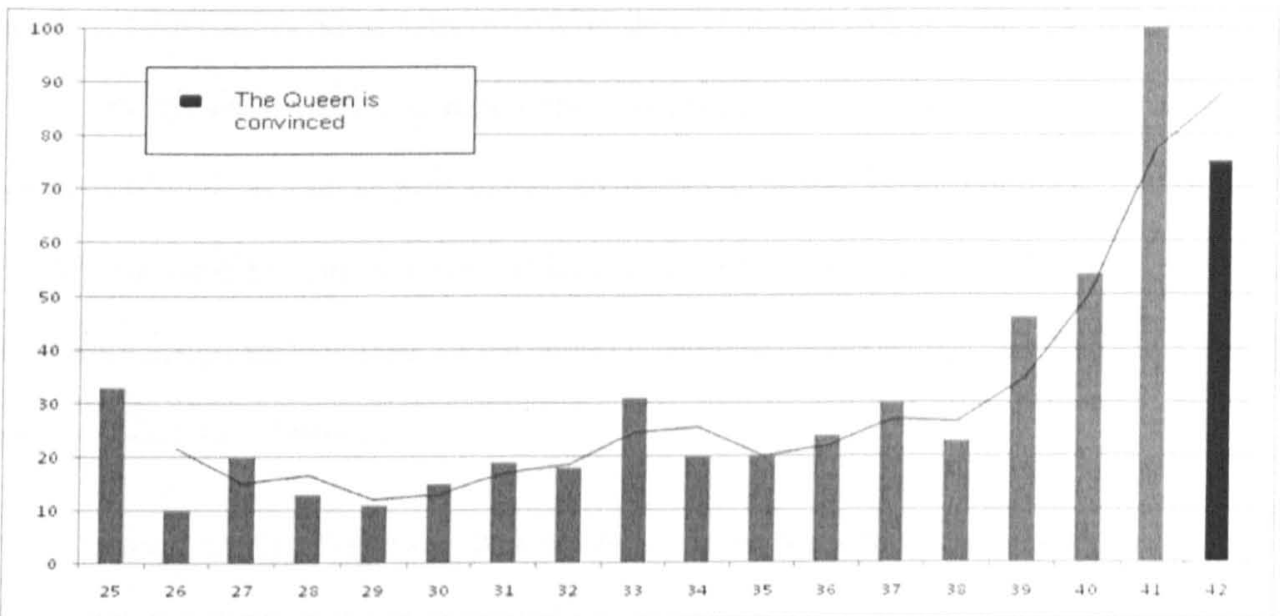

$\mathrm{Y}$ axis $=$ frequency (shot length in seconds $/ 60$ seconds)

$\mathrm{X}$ axis $=$ shot number

All of these signs here give validity to Zhang's argument, and make it conceivable that the queen would change her originally pacifist position to a martial one.

Figure 30: Zhang Qian Conjures up Images of War to Convince a Pacifist (HWDD 44-03)

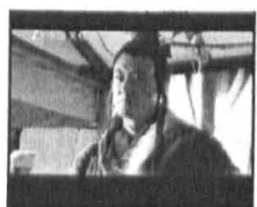

HWDD 44-03-31

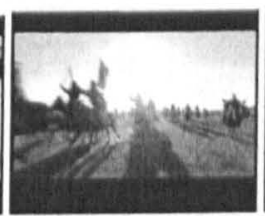

HWDD 44-03-32

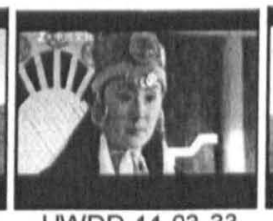

HWDD 44-03-33

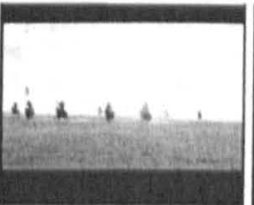

HWDD 44-03-34

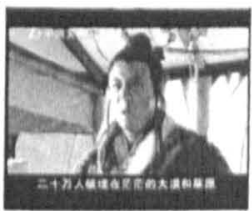

HWDD 44-03-35 
Following this piece of dialogue, the queen agrees to call in a meeting with the royal elders to discuss a possible alliance with the Han. This meeting is devised as a lengthy dialogue sequence between the various royal elders, set at the court of the Indo-Scyth queen (HWDD 44-07). The sequence is arranged without strong visual signs: shots of the various elders are juxtaposed with each other to show who is speaking and what the other discussants' response is, and medium close-up shots of the queen and Zhang Qian are used to show the two characters' reaction to the ongoing debate (Zhang's support for the pro-war arguments is demonstrated by corresponding gestures and facial expressions, while the queen's support for the same arguments is signified by her audibly sighing when the opposition makes their case). At random intervals, extremely long shots show the hall in which the debate is taking place. The dynamics generally follow the conversation, and the acoustic signs (two sections of oriental music as well as bird sounds throughout the first section of the sequence) merely provide atmosphere, but do not contribute to the discussion on war and peace. In this sense, the maximum stress is placed on the verbal statements made by the five discussants, which are reproduced in Figure 31 .

Figure 31: The Debate on War and Peace at the Indo-Scyth Court (HWDD 44-07)

\begin{tabular}{|c|c|c|c|}
\hline $\begin{array}{l}\text { Shot } \\
\text { No. }\end{array}$ & $\begin{array}{l}\text { Chinese } \\
\text { Original }\end{array}$ & $\begin{array}{l}\text { English } \\
\text { Translation }\end{array}$ & $\begin{array}{l}\text { Main } \\
\text { Arguments }\end{array}$ \\
\hline 01-08 & $\begin{array}{l}\text { 金侯甲: 女王, 我认为 } \\
\text { 此举可行。匈奴和月氏 } \\
\text { 水火不相容, 月氏两大 } \\
\text { 期难, 先王的耻序, 我 } \\
\text { 们必须时时刻刻记在心 } \\
\text { 里! 今汉朝挥师北伐, } \\
\text { 正是东西夹击歼灭匈奴 } \\
\text { 报仇雪恨的好时机。我 } \\
\text { 建议我们应该出兵消灭 } \\
\text { 匈奴, 如果我们错失了 } \\
\text { 这个机会的话, 将憁对 } \\
\text { 祖先和子孙, 下决心 } \\
\text { 吧! 我愿意做先锋。 }\end{array}$ & $\begin{array}{l}\text { Marquis 1: Your majesty, I think this } \\
\text { undertaking is feasible. The Xiongnu } \\
\text { and the Indo-Scyths are as } \\
\text { incompatible as fire and water. We } \\
\text { must always remember the two } \\
\text { calamities that befell the Indo-Scyths as } \\
\text { well as the humiliation of the former } \\
\text { king. Now that the Han dynasty is } \\
\text { marching their troops to war on a } \\
\text { northern expedition, the right time has } \\
\text { come to take revenge and annihilate } \\
\text { the Xiongnu by attacking them from } \\
\text { both east and west. I suggest we } \\
\text { dispatch our troops and exterminate the } \\
\text { Xiongnu. If we pass up this opportunity, } \\
\text { it will be to the shame of our ancestors } \\
\text { and descendants. Let us make a } \\
\text { decision! I would like to take the lead. }\end{array}$ & $\begin{array}{l}\text { - The Indo-Scyths and the } \\
\text { Huns are natural } \\
\text { adversaries } \\
\text { The Indo-Scyths have } \\
\text { suffered humiliation at the } \\
\text { hands of the Huns. } \\
\text { The Han dynasty's war } \\
\text { against the Huns is an } \\
\text { opportunity to exert } \\
\text { revenge } \\
\text { A two-front war would } \\
\text { annihilate the Huns } \\
\text { Not going to war would be } \\
\text { a disgrace to the } \\
\text { ancestors and to posterity }\end{array}$ \\
\hline
\end{tabular}




\begin{tabular}{|c|c|c|c|}
\hline $32-37$ & $\begin{array}{l}\text { 金侯甲: 双靡㓣候, 要 } \\
\text { 居安思危, 匈奴不是一 } \\
\text { 次的攻击我们, 而是不 } \\
\text { 断的攻击, 他们已经使 } \\
\text { 月氏经历两次大劫难。 } \\
\text { 为了不出现第三次大劫 } \\
\text { 难的开始, 现在正是好 } \\
\text { 时机。你不要以为匈奴 } \\
\text { 离我们远就平安无事 } \\
\text { 了! 忽岭西现在又出现 } \\
\text { 了囟奴骑兵。 }\end{array}$ & $\begin{array}{l}\text { Marquis 1: Marquis of Shuangmi, we } \\
\text { have to be vigilant in peace times. The } \\
\text { Xiongnu did not attack us only once, } \\
\text { but relentlessly. They have twice } \\
\text { already caused us massive calamity. If } \\
\text { we want to prevent the start of a third } \\
\text { great calamity, now is the right } \\
\text { opportunity. You should not assume } \\
\text { that all is well just because the Xiongnu } \\
\text { are far away. Xiongnu riders have again } \\
\text { been sighted in the west of the Ridge of } \\
\text { Asia. }\end{array}$ & $\begin{array}{l}\text { Peace is not a permanent } \\
\text { state; it is fragile and must } \\
\text { be guarded constantly } \\
\text { - The Indo-Scyths have } \\
\text { suffered humiliation at the } \\
\text { hands of the Huns } \\
\text { - The Han dynasty's war } \\
\text { against the Huns is an } \\
\text { opportunity to exert } \\
\text { revenge } \\
\text { - The Huns are always } \\
\text { dangerous, no matter how } \\
\text { far away they are }\end{array}$ \\
\hline \multicolumn{4}{|c|}{ Peace Supporters } \\
\hline $\begin{array}{l}\text { Shot } \\
\text { No. }\end{array}$ & $\begin{array}{l}\text { Chinese } \\
\text { Original }\end{array}$ & $\begin{array}{l}\text { English } \\
\text { Translation }\end{array}$ & $\begin{array}{l}\text { Main } \\
\text { Arguments }\end{array}$ \\
\hline 14-31 & 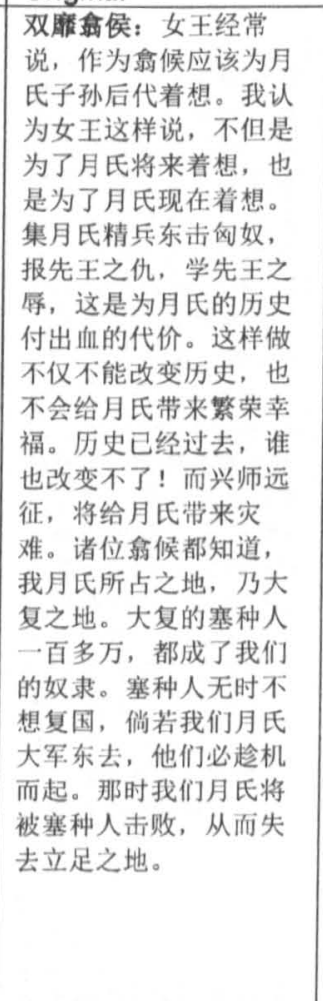 & $\begin{array}{l}\text { Marquis of Shuangmi: Your majesty } \\
\text { has often said that to be a Marquis one } \\
\text { has to consider posterity. I take it you } \\
\text { majesty did not only mean that we } \\
\text { should consider the future, but also that } \\
\text { we should consider the present. To } \\
\text { gather our crack troops and attack the } \\
\text { Xiongnu in the east to avenge the } \\
\text { former king and right his humiliation } \\
\text { would be a historic tribute for which the } \\
\text { Indo-Scyths would pay in blood. Not } \\
\text { only would such an action not change } \\
\text { history, it would also not bring } \\
\text { prosperity and happiness to the Indo- } \\
\text { Scyths. History lies in the past, and no } \\
\text { one can change it! Furthermore, to } \\
\text { dispatch troops on an expedition would } \\
\text { bring calamity to the Indo-Scyths. Sirs, } \\
\text { you all know that the territory that the } \\
\text { Indo-Scyths occupy is the Daxia } \\
\text { territory. The ethnic people in the } \\
\text { border regions of Daxia have a } \\
\text { population of over } 1,000,000, \text { which we } \\
\text { all enslaved. The people in the border } \\
\text { regions could at any time unexpectedly } \\
\text { attempt to reclaim their nation. Should } \\
\text { we move our troops eastwards, they } \\
\text { would most certainly cease the } \\
\text { opportunity and rebel. In that case, we } \\
\text { Indo-Scyths would be vanquished by } \\
\text { the border people, thus losing our } \\
\text { foothold. }\end{array}$ & $\begin{array}{l}\text { To avenge a past } \\
\text { humiliation would only } \\
\text { serve to right a historical } \\
\text { wrong, but would not } \\
\text { benefit the people of the } \\
\text { present } \\
\text { - A war would cause } \\
\text { suffering for the Indo- } \\
\text { Scyths } \\
\text { - History cannot be } \\
\text { changed } \\
\text { - A war would destabilise } \\
\text { the domestic relations with } \\
\text { local ethnic minorities }\end{array}$ \\
\hline $38-42$ & $\begin{array}{l}\text { 双庣萮侯: 方才女王已 } \\
\text { 经说了, 匈奴正与汉军 } \\
\text { 作战, 两强相争。匈奴 } \\
\text { 岂能份军西征。这明摆 } \\
\text { 这是不可能的, 你所见 } \\
\text { 到的匈奴, 是小股匈 } \\
\text { 奴, 并不过数百, 不足 } \\
\text { 为患。 }\end{array}$ & $\begin{array}{l}\text { Marquis of Shuangmi: As the queen } \\
\text { has already said, the Xiongnu are at } \\
\text { war with the Han troops. It is a fight } \\
\text { between two great powers. How could } \\
\text { the Xiongnu divide their forces and } \\
\text { march westwards. That is clearly not } \\
\text { possible. The Xiongnu that you } \\
\text { [Marquis 1] saw are small bands of no } \\
\text { more than } 100 \text { men. They cannot do us } \\
\text { any harm. }\end{array}$ & $\begin{array}{l}\text { If the Huns are occupied } \\
\text { with their war against the } \\
\text { Han, they will not attack } \\
\text { the Indo-Scyths }\end{array}$ \\
\hline
\end{tabular}




\begin{tabular}{|c|c|c|c|}
\hline $49-59$ & $\begin{array}{l}\text { 费霜峇侯: 女王, 未来 } \\
\text { 征报世界的不是军队而 } \\
\text { 是商队。战争从来没有 } \\
\text { 给各民族带来幸福。只 } \\
\text { 有墑队才能在各民族之 } \\
\text { 间架起友谊的桥梁。女 } \\
\text { 王, 不要议什么东征西 } \\
\text { 伐, 汉使既然不远千里 } \\
\text { 来到了月氏, 我们就议 } \\
\text { 定两国互派多少商队 } \\
\text { 吧。女王陛下, 汉朝的 } \\
\text { 丝绸可是稀世珍奇, 远 } \\
\text { 道罗马一元值十斤黄 } \\
\text { 金。可是我听说在汉朝 } \\
\text { 的长安, 一批丝绸只值 } \\
\text { 一两黄金。 }\end{array}$ & $\begin{array}{l}\text { Marquis of Guishuang: Your majesty, } \\
\text { in the future the world will not depend } \\
\text { upon armies but on trade caravans. } \\
\text { War has never brought happiness to } \\
\text { any people. Only trade caravans will be } \\
\text { able to build bridges of friendship } \\
\text { between all ethnic groups. Your } \\
\text { majesty should not be discussing a } \\
\text { westward migration and attack. Since } \\
\text { the Han envoy has come so far to visit } \\
\text { the Indo-Scyths, we should decide how } \\
\text { many trade caravans our two states will } \\
\text { be exchanging. Your majesty, the Han } \\
\text { dynasty's silk is a rare commodity for } \\
\text { which faraway places like Rome will } \\
\text { pay } 10 \text { pounds of gold per load. } \\
\text { However I have heard that a batch of } \\
\text { silk only costs } 50 \text { grams of gold in } \\
\text { Chang'an. }\end{array}$ & $\begin{array}{l}\text { - In the future, trade will be } \\
\text { more important than } \\
\text { warfare } \\
\text { - War causes suffering for } \\
\text { the people } \\
\text { - Trade creates friendship } \\
\text { between ethnically diverse } \\
\text { groups } \\
\text { Chinese silk is a precious } \\
\text { commodity worth trading } \\
\text { for }\end{array}$ \\
\hline $60-70$ & $\begin{array}{l}\text { 畣侯丁: 老人们倒是常 } \\
\text { 常想念祁连、敦煌, 可 } \\
\text { 是要是让他们回去, 他 } \\
\text { 们却不愿意了。因为那 } \\
\text { 里没有这里富饶。在这 } \\
\text { 里, 我们有肥沃的土地 } \\
\text { 可以耕作 [对白剧本: 我 } \\
\text { 们有塞种奴隶可供驱 } \\
\text { 使], 每天的宴会都十分 } \\
\text { 的丰盛。有大盘的牛 } \\
\text { 肉, 还有丰腉的牧场 [对 } \\
\text { 白剧本: 席前有塞种舞 } \\
\text { 女歌舞], 更有美丽的雪 } \\
\text { 山 [对白剧本: 帐内有希 } \\
\text { 腊女奴伺候]。重新拥有 } \\
\text { 这些容易吗 [对白剧本: } \\
\text { 这些在祁连山敦煌有 } \\
\text { 吗]? 是非常不容易的 } \\
\text { [对白剧本: 一样也没 } \\
\text { 有]。女王, 民意不可 } \\
\text { 违, 众怒难犯。千平万不 } \\
\text { 可兴师东攻匈奴 } \\
\text { 举族东迁! }\end{array}$ & $\begin{array}{l}\text { Marquis 4: Older people will often } \\
\text { remember with longing Qilian and } \\
\text { Dunhuang, but when you ask them to } \\
\text { return they actually do not wish to do } \\
\text { so. That is because those places are } \\
\text { not as richly endowed as we are here. } \\
\text { Here, we have fertile soil that we can } \\
\text { cultivate [original script: we have slaves } \\
\text { from the border people at our demand], } \\
\text { and have extremely rich banquets } \\
\text { every day. We have huge plates of beef } \\
\text { and we also have rich and fertile } \\
\text { grazing land [original script: before } \\
\text { each banquet we have dancer girls } \\
\text { from the border regions perform songs } \\
\text { and dances for us] and what's more, we } \\
\text { have beautiful snow-capped mountains } \\
\text { [original script: and in our tents we have } \\
\text { slave girls from Greece waiting for us]. } \\
\text { Is it likely that we would possess such } \\
\text { things again [original script: would we } \\
\text { have that in Dunhuang and the Qilian } \\
\text { mountain range]? That would be } \\
\text { extremely difficult [original script: it } \\
\text { wouldn't be the same]. Your majesty, } \\
\text { the popular will should not be } \\
\text { disobeyed. It is dangerous to anger the } \\
\text { masses. We can absolutely not attack } \\
\text { the Xiongnu and migrate eastwards! }\end{array}$ & $\begin{array}{l}\text { - Although older Indo- } \\
\text { Scyths have fond } \\
\text { memories of their } \\
\text { homeland, those lands are } \\
\text { not as fertile as the } \\
\text { present ones } \\
\text { - Returning to the Indo- } \\
\text { Scyth homeland would } \\
\text { mean to lose the current } \\
\text { state of prosperity } \\
\text { Foreign policy should take } \\
\text { into consideration public } \\
\text { opinion and be in the } \\
\text { interest of the people }\end{array}$ \\
\hline $70-77$ & $\begin{array}{l}\text { 鸽侯戊: 匈奴, 轻视不 } \\
\text { 得。十几年前我们拥兵 } \\
\text { 二十万, 自恃强盛而轻 } \\
\text { 视匈奴, 结果被匈奴打 } \\
\text { 败 [就是其出兵一半也强 } \\
\text { 过我军]。现在我们拥兵 } \\
\text { 二十万, 而匈奴已有六 } \\
\text { 十万骑兵。就是其出兵 } \\
\text { 一半, 也强过我军。那 } \\
\text { 些认为与汉朝联合就可 } \\
\text { 以打败仦奴, 那完全是 } \\
\text { 一厢情愿的梦想。 }\end{array}$ & $\begin{array}{l}\text { Marquis 5: The Xiongnu must not be } \\
\text { slighted. Just over ten years ago, we } \\
\text { moved } 20,000 \text { troops against the } \\
\text { Xiongnu. Confident of our strength, we } \\
\text { underestimated the Xiongnu and as a } \\
\text { result, we were defeated by them } \\
\text { [original script: who defeated our troops } \\
\text { with an army half the size]. Now } \\
\text { currently we have } 200,000 \text { men at our } \\
\text { disposal, but the Xiongnu already have } \\
600,000 \text { riders. That is half of the troops } \\
\text { they dispatched, and still they are } \\
\text { stronger than our army. To believe that } \\
\text { we could join forces with the Han } \\
\text { dynasty and defeat the Xiongnu is } \\
\text { entirely wishful thinking. }\end{array}$ & $\begin{array}{l}\text { - The Huns are too strong } \\
\text { an adversary } \\
\text { - A victory over the Huns is } \\
\text { not realistic }\end{array}$ \\
\hline
\end{tabular}


The overwhelming number of shots in this sequence is clearly devoted to the opposition of a military alliance with the Han dynasty: of the seventy-seven shots that compose the debate, 51 show the opposition making its case $(66 \%)$, while only fourteen are devoted to the sole supporter of an attack on the Huns (18\%); the other twelve shots either contain no dialogue or have the queen asking for the opinions of individual royal elders (16\%). Those opposing an alliance draw from various different discourse strands in order to make their point (for instance the welfare discourse, the public security discourse and the national identity discourse, suggesting that war would have adverse affects on each of these areas). The main discursive statements that are made in this dialogue are:

- War is a matter of honour.

- Peace is not a permanent state; it is fragile and must be guarded constantly.

- Historical defeats lie in the past and cannot be changed.

- Foreign security policy should be in the service of the people.

- Wars destabilise a state domestically.

- Wars cause suffering for the people.

- Trade, that is the mutually beneficial exchange of rare goods between friendly societies, facilitates peace.

The conclusion of the debate is then relatively clear: the queen of the Indo-Scyth has to grudgingly give in to the majority and respectfully decline Zhang Qian's offer. She does, however, offer Zhang the secret formula to the steal compound used in forging the IndoScyth swords (shot 84). Also, with the debate touching upon the importance of trade relations between the Indo-Scyths and the Han dynasty (including a cross-cut to Zhang Qian nodding in agreement; shot 56), it is suggested that Zhang was indeed able to establish such relations. 
This then concludes Zhang Qian's diplomatic effort in the West, and considering that the main discursive position on foreign security throughout HWDD is a martial one that gives priority to war over peace, it may be surprising that Zhang's failure to recruit allies for the war against the Han does not cause his mission to the West to be interpreted as a failure. The reason for this is not only that Zhang was still able to contribute to the war effort at home (securing the Fergana horses and the Indo-Scyth swords for the Han army), but more importantly the fact that Zhang's voyage has received a new significance throughout the sequences discussed above: the actual achievement is Zhang's establishment of trade relations with amiable Central-Asian states. This is also the reason why the pacifist argument of the Indo-Scyth is not ridiculed here: it is on one hand the position of a future friend and ally, and on the other hand it fundamentally contributed to the formation of the new trade relations. But even though the discursive statements on trade and foreign security (particularly that trade facilitates peaceful relations between ethnically diverse groups) present such an outcome as valuable in itself, the true reason why Zhang's voyage to the West is in the end viewed as successful is that his achievements are linked to the discourse on Chinese national identity. This is achieved by presenting Zhang's voyage with a high degree of pathos, and repeatedly clarifying that he was the one to essentially 'invent' the Silk Roads. These Silk Roads are then interpreted as part of the Han Chinese heritage; something that the audience should feel proud of. The three central moments in the drama that assure this effect are (see also Figure 32):

1. Sequence HWDD 26-17: Zhang Qian says his farewell to the emperor and sets out on his voyage. The dynamics lead up to the moment when the emperor gestures Zhang to rise after he had bowed down, and the rest of the sequence then shows Zhang walking off, and finally riding off with his entourage, while the emperor looks after him in reverence. A romantic theme plays throughout, and low angels are repeatedly 
used to relay the glory of both Zhang Qian and the emperor. The sequence concludes with the narrator stating the following:

Narrator: In order to fulfill Liu Che's grand strategy of establishing contacts with potential allies, Zhang Qian and his meticulously chosen entourage of over one-hundred men secretly left Chang' an and marched westwards. They would cut across the Xiongnu territory and seek out the nations of Central Asia. Two thousand years later, all historians of the world would regard this small special group of envoys as the pioneers who established the Silk Roads, which would connect Europe and Asia. But as this tiny group disappeared into the distance leaving Chang' an and departing for out-of-the-way places, on a journey that would last more than ten harsh years, the Han Emperor Liu Che who had dispatched this group essentially did not know whether Zhang Qian's team would ever return.

旁白: 为实现刘彻结交盟友的宏伟战略意图, 张㥶及其精心挑选的一百多 名随从人员, 秘密地由长安向西进发。他们将穿越匈奴地域, 去寻 找大月氏国。两千多年后, 全世界的历史学家们把这次出使的这支 小分队，视作开拓西域建立连通欧亚之间丝绸之路的发端。但是当 目送这支小小的队伍离开长安城走向绝域一一即将开始长达十多年 的苦难征程时, 发起这次远行的汉武帝刘彻, 当时却根本不知道, 张㥶一行, 是否还真的能够回来?

Note in particular how the claim that Zhang Qian linked China with Europe by establishing the Silk Roads is here legitimized with the statement that "all historians of the world" agree on this issue.

2. Sequence HWDD 44-02, scene 2 (shots 24 - 51): Zhang Qian arrives at the Indo-Scyth border. There, he falls to his knees and calls out to the emperor, swearing his undying loyalty to the Han cause (note in particular how the melodramatic music and the shot dynamics maximise the emotional gravity of Zhang Qian falling to his knees; shots 38-44). The sequence ends with Zhang staring into the distance and starting to cry (shot 51), while his entourage is arranged in the background.

3. Sequence HWDD 45-19: Zhang Qian returns to Chang'an after 13 years of hardship. The emotional impact of Zhang's arrival before the emperor has already been 
discussed (see 4.1.i), but it is worth reproducing here the narrator's summary of

Zhang's voyage (shots 98 - 102):

Narrator: In the third year of the Yuanshuo era, Zhang Qian finally returned to Chang'an from the western territories. Although he had failed to complete his assigned mission, but as an emissary of the Han dynasty his errands had taken him ten thousand miles, and he brought back with him geographical knowledge that many of his compatriots simply could not fathom. No matter whether for the Han Emperor $\mathrm{Wu}$ or for Chinese people who wished to understand the world, Zhang Qian's actions had a pioneering significance. From that time onward, there would always be men who travelled west with the sun, from the east to the central plains, across wide ranges of mountains and deserts, to finally establish the Silk Roads, which would shine brightly in the historical annals for two thousand years.

旁白：元朔三年, 张寒终于自西域回到长安。显然未能完成预期使命, 但 作为汉朝使臣, 细行数万里, 带回了许多国人过去根本无从知道的 地理知识。无论对于汉武帝还是对于有心了解世界的中国人，张赛 此行都具有丵空拓荒的意义。从此之后, 不断有人西出阳光, 由东 归中土, 跨越重重关山大漠, 终于踏出了一条辉炳史册两千年的丝. 绸之路。

Here, the extreme notion that Zhang established the Silk Roads himself is softened slightly by the statement that others followed his trails to create the famous trade routes, but Zhang's pioneering influence is nonetheless stressed. Note how the sequence ends with the Emperor staring off into the distance, solemnly saying about Zhang "A hero! You are a hero of our great Han dynasty" (yingxiong! ni shi wo da Hanchao de yingxiong! 英雄！你是我大汉朝的英雄！ shot 102), thereby leaving no room for misinterpretation of how significant Zhang's achievements are. 


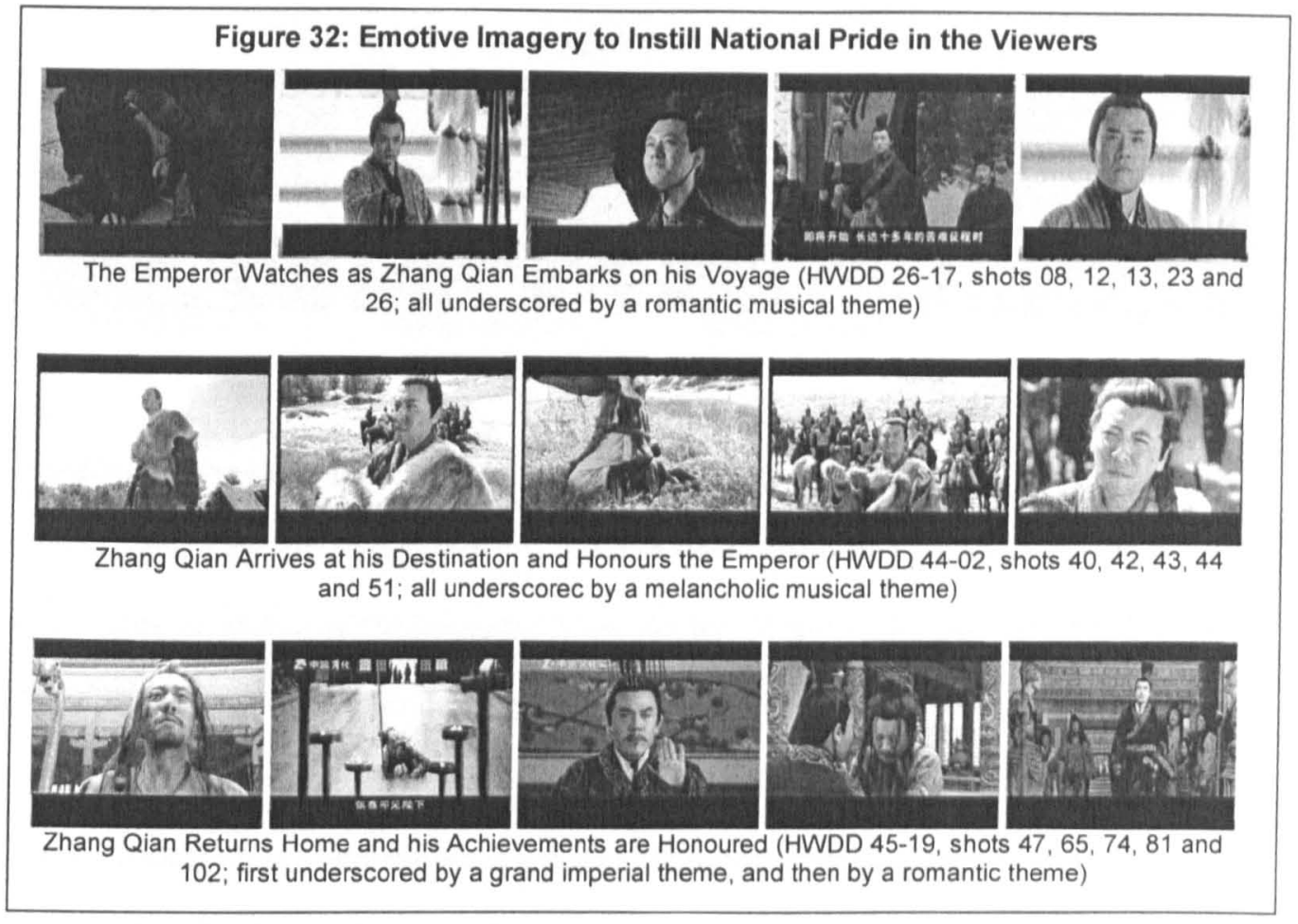

All of these sequences have one thing in common, and that is the use of extreme pathos in relation with the heroic exploits of Zhang Qian. This is the same mechanism that also functions to legitimize throughout the drama series what might otherwise be conceived as warmongering on the part of the Han emperor. His battles against the Huns are justified in the end by the expansion of the Han nation, which is presented in heavily pathos-laden sequences such as HWDD 58-14. Even the demise of the Huns, who functioned as the antagonistic force throughout the drama, is used to instil pride in the viewer. This is most strikingly exemplified by the last sentence of the drama series (HWDD 58-15), in which the narrator concludes:

Narrator: The lifeblood of the Huns has now merged with the blood vessels of the great Chinese family of ethnic groups.

旁白: 在今日中华民族大家庭的血脉中, 也融进了匈奴民族的血被。 


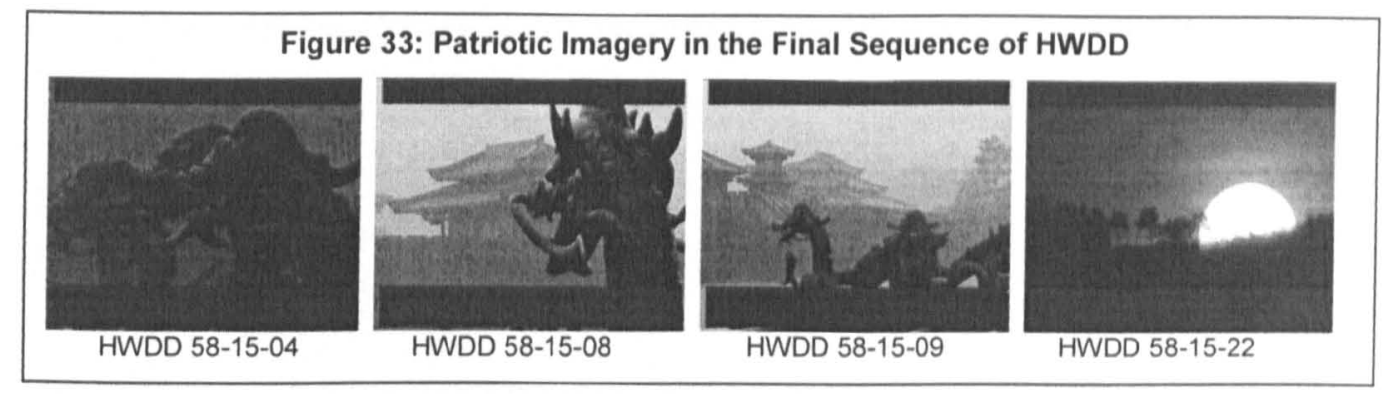

The disconcerting use of the eugenic discourse in this verbal statement is then juxtaposed with images of the imperial palace in a thunder storm (including angles that prominently feature dragon statues in the foreground; see Figure 33) and with a powerful imperial music theme. The last shot of the drama is a blazing red setting sun. This arrangement is clearly meant to give the assimilation of the Huns by the Han Chinese a patriotic connotation, thereby contributing to the notion of a great Chinese heritage that literally all Chinese viewers share "in their veins" (mai 脉).

To summarise, the foreign security discourse in HWDD is primarily martial in nature. Warfare is depicted as a legitimate tool to protect the nation from foreign aggressors. Discursive statements on the barbaric nature of the aggressor, as well as on the weakness of pacifists, help to further justify military conflict as a solution to foreign security threats.

Diplomacy is ostensibly given the same priority as warfare, but the relation between the two foreign security approaches is put into perspective on one hand by the fact that the Han court understands diplomacy merely as the recruitment of military allies, and on the other hand by the enormous amount of drama sequences that deal with warfare instead of foreign cooperation.

Discourse fragments on peace are also presented in this drama, and are interlinked with the welfare discourse, repeatedly stating that foreign security policy should be in the service of the people and that wars exhaust the population. In addition, trade is presented as a viable 
form of state interaction that is said to promote the amiable relations between ethnically diverse groups. This last discourse strand is developed primarily in the narrative arc that shows the 'foundation' of the Silk Roads, and helps sanction the achievements of the alleged founder of these trade routes, Zhang Qian.

Both the discourse on war and the one on peace (or rather: on the establishment of peaceful trade relations) are presented with an abundance of pathos. The outcomes of the war as well as of Zhang Qian's diplomatic mission are equally presented as national achievements of the Han dynasty, and are used to instil patriotic pride in the audience. In this sense the martial means of conducting foreign security policy are justified by the ends, i.e. by the expansion of the great Han Chinese nation.

\subsection{Visual Political Discourses in a Main-Melody Crime Drama}

Next in my analysis, I shall turn to the governance and security discourses in a modern crime drama. For this purpose, the series GAJZ was chosen (Jin, 2003). The twenty-episode series is a 'main-melody' drama, i.e. an official state propaganda series, commissioned by the top functionaries of the Public Security Ministry (Gonganbu 公安部) in order to improve the public image of the police (113, 07:56 - 08:19). The production details were handled by the Ministry's production unit, the Golden Shield centre (Gongan bu jindun yingshi wenhua zhongxin 公安部金盾影视文化中心), which possesses its own filming permit; CCTV followed as a second production company, and a Shenzhen construction company involved in visa services at the Hong Kong-Shenzhen border joined as a financier (113, 65:33 - 71:07). The script was written by a former director of the Public Security Ministry, Zhu Entao 朱恩涛, as well as his wife, the journalist Yang $\mathrm{Zi}$ 杨子. The drama was directed by Jin Tao 金搯, and featured the renowned television actor Pu Cunxin 㴖存昕 in the main role. The series aired on CCTV-1, starting on 26 August 2003, and was broadcasted each evening during primetime; it ranked as the $4^{\text {th }}$ most popular television drama of 2003 (Wang, 2004, 249). The drama 
tells the story of Commissioner Li Jian 黎剑, who is transferred to the fictional north-eastern Chinese city of Yundu 云都 (roughly corresponding to the city of Dalian 大连 where the drama was filmed) to solve a recent crime case and reform the local law enforcement system. According to one interviewee, the drama was based on the original (yuanxing 原形) experiences of six commissioners and various police officers who were interviewed by the screenwriters (I13, 02:24 - 15:07 and 22:48 - 23:34). The main theme of the drama by default is public security, but the repeated interaction between Li Jian and his party and government superior Cheng Zhi 程志 also offers strong references to the discourse strand on governance. The discursive statements identified in this analysis, as well as the signs used to construct these statements, are summarised in the corresponding tables in Appendix 3.

\subsection{Responsible and Profẹssional Leadership}

The main melody drama GAJZ and the historical drama HWDD analysed above have in common that they both focus strongly on those who govern, and those who are being governed. However, the discourse fragments on the state are communicated differently in the crime drama than they are in HWDD. In general, the governing theme is personified and hence channelled by the head of the CCP's provincial Political \& Law Committee (zhengfawei 政法委), secretary Cheng Zhi 程志, who is Commissioner Li Jian's superior, and represents the provincial government and party leadership. For the purpose of analysing the discourse on governance and leadership in GAJZ, I am focusing here on the discursive statements that I was able to isolate concerning the character of Secretary Cheng, his function as a superior to Commissioner $\mathrm{Li}$ and his role as a leader to the people of Yundu city.

Cheng Zhi's function as a leadership figure is generally not emphasised very strongly through visual signs. One exception is the first brief appearance of the character in GAJZ 0111. The sequence shows Deputy Commissioner Huo Xiang 焦样 and his men handling a hostage situation at the cruise ship SS Oriana. After a fifth of the sequence, Secretary Cheng 
arrives. The following two scenes (shots 32 - 46) are then used to introduce the character for the first time in the drama, and establish him as a proactive leader. Upon arrival (first scene; shots 32 and 33) Secretary Cheng's immediately attempts to obtain a better picture of the situation. The discursive statement made here is that the party leadership is taking charge of a potentially dangerous situation. The character's first line of dialogue is: "Huo, let's go up there and have a look." It is then made clear through additional dialogue that such an action would be unsafe. The secretary, however, ignores the cautioning officers and makes his way towards the cruise ship, accompanied by the two deputy commissioners Huo Xiang and Dai Wenqing 堹文清, as well as a heavily armed police escort (second scene; shots $34-45$ ). The walk across the quay bridge is then used to make additional statements concerning Cheng's role as a leader (Figure 34 ). This is achieved by layering various visual, acoustic and verbal signs.

Visually, the character Cheng Zhi is placed in the centre of the frame, flanked by the armed police officers, and backed by Huo and Dai (shots $34-37,39-41$ and $43-45$ ). All shots except for one are frontal shots of Cheng and the men surrounding him (the exception is shot 36, which shows the men from the side). Low camera angels force the viewers to look up to the characters (shots 35,36 , and 40). All of these signs work together to emphasise Cheng's authority.

The general sense of urgency already established through the context (hostage situation) and the early shots of the sequence (especially shots $10-16$ and $18-28$, in which armed police officers storm the quay and get into firing position) is further heightened by shaky hand camera movement throughout the scene and a high shot frequency (the average length of shots $34-37,39-41$ and $43-45$ is 3.5 seconds; shots 38 and 42 show a different scene and are therefore not included in this calculation). Tensions are further raised acoustically, 

be heard in the background.

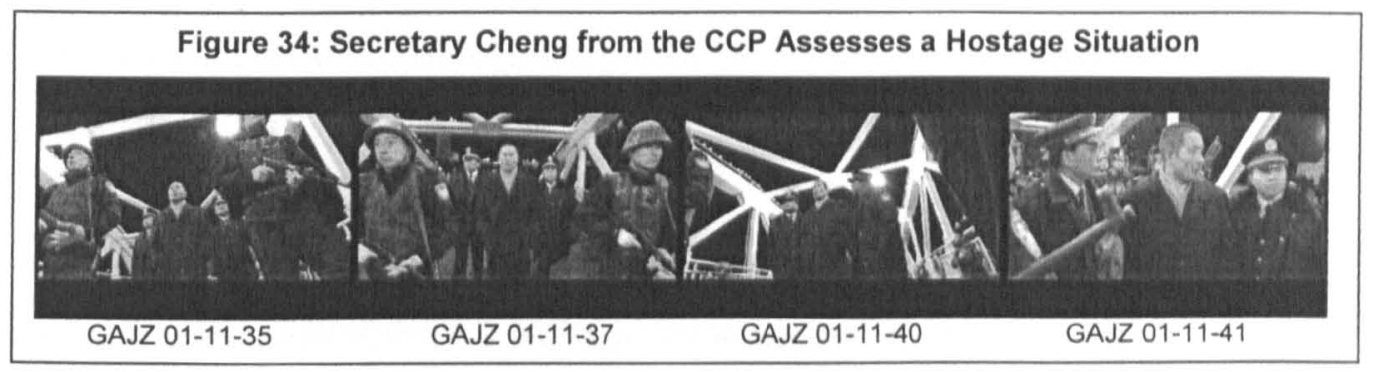

As the men arrive at the other side of the bride, Cheng Zhi gives the following orders to Huo Xiang in shots $39-41$ (the character makes a very similar verbal statement later in the sequence, during shots 75 and 76 ):

Cheng Zhi: Regardless of the measures you take, you have to guarantee the hostages' safety. If you can spare the hostage takers' lives, then do so. If that's really not possible, then kill them.

程志: 不管采取什么措施一定要保证人质安全, 劫持人质的歹徒能留活口 留活口。实在不行就干死他。

To sum up, the combination of these verbal, acoustic and visual signs formulates of the following discourse fragments:

- The party leader wields authority.

- The party leader is capable of assessing a dangerous crime situation.

- The party leader is proactive.

- The party leader is fearless.

- The party leader has zero tolerance towards criminals.

- The party leader cares more about the safety of the general populace than about his own wellbeing.

Other than in this sequence, most discursive statements made by or about the character Cheng Zhi are verbal in nature and feature very few visual signs. The majority of such verbal 
discursive statements are made in dialogue sequences between the secretary and

Commissioner Li Jian (see GAJZ 04-13; 04-16; 04-19; 6-14; 10-10; 14-14; 16-02; 17-20; 18-01;

and 19-01). As a representative example, I have provided a sequence protocol of GAJZ 04-16.

The sequence consists of one scene, which takes place in the office of secretary Cheng, and shows Li Jian reporting back to Cheng Zhi on the progress of his crime investigation.

The arrangement of this scene is entirely conventional (Figure 35). All shots except for one are medium close-ups at an eye-level angle, showing either one of the two characters. Camera movement is kept to a minimum: Camera actions such as pan and tilt are used occasionally, and are consequently marked in the protocol, but these movements are merely very minor corrections to keep the respective characters in the frame as they move their bodies during the conversation. The only shot that somewhat deviates from this norm is a long shot of the two men talking to each other across secretary Cheng's office desk (shot 13). However, this perspective is also a conventional part of dialogue scenes, functioning as the main shot, i.e. the shot that shows the line of sight between the characters, and gives an impression of the general setting.

Other aspects of the scene also do not constitute particular signs purveying meaning. The sequence does not feature any music or any sound effects, and the dynamics generally follow the flow of the conversation (lengthy shots showing the person who is speaking, juxtaposed with short shots showing the character who is listening). The shot frequency slopes downwards towards the middle of the scene (the dynamics reaching the slowest point roughly when the two characters are shown facing each other in shot 13), and then slopes upward again as the conversation picks up some momentum. However, these dynamics do not underscore a particularly important part of the dialogue or action.

The only visual signs that the viewer is provided with are the objects and persons in the scene and the way they are arranged to each other. The physical objects that are shown 
indicate that the action is set in a government office: a Chinese flag and a calligraphy provide the background for the shots featuring Secretary Cheng, who is wearing a dark suit; a map of China and a green plant can be seen in the shots that show commissioner $\mathrm{Li}$, who is wearing a police uniform. Otherwise, however, the arrangement of objects and personage in the scene is as functional as the shot angles and editing dynamics.

In this sense, everything in this or the other dialogue scenes between $\mathrm{Li}$ Jian and Cheng Zhi is geared towards keeping the viewer's attention on the verbal statements. I have translated below five sections of dialogue from this and similar sequences in order to show what discourse fragments are presented in these types of conversations (Figure 36).

Figure 36: Discourse Fragments in the Dlalogues between Commissioner and Secretary.

\begin{tabular}{|c|c|c|c|}
\hline $\begin{array}{l}\text { Sequence No. } \\
\text { and content }\end{array}$ & $\begin{array}{l}\text { Chinese } \\
\text { Original }\end{array}$ & \begin{tabular}{|l|} 
English \\
Transiation \\
\end{tabular} & $\begin{array}{l}\text { Discourse } \\
\text { Fragments }\end{array}$ \\
\hline $\begin{array}{l}\text { GAJZ 04-16: } \\
\text { Li Jlan reports } \\
\text { back to Cheng } \\
\text { Zhi on his } \\
\text { progress with a } \\
\text { crime } \\
\text { investigation. }\end{array}$ & 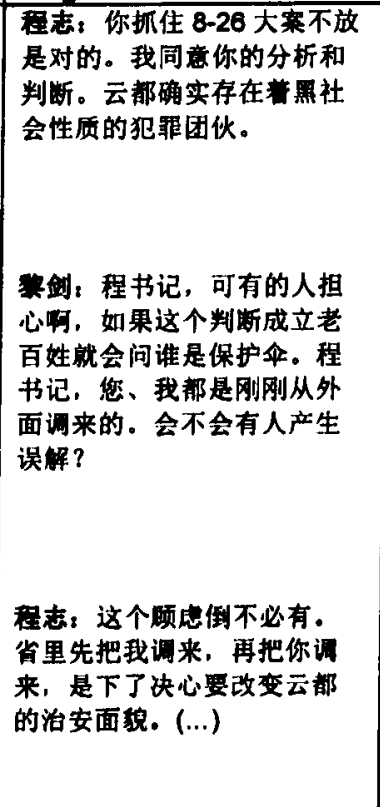 & $\begin{array}{l}\text { Cheng Zhi: To tackle the case } \\
\text { of } 26 \text { August was the right } \\
\text { thing to do. I agree with your } \\
\text { analysis and assessment. We } \\
\text { definitely have a criminal } \\
\text { underworld group at work in } \\
\text { Yundu. } \\
\text { LI Jian: Secretary Cheng, } \\
\text { there may be some who fear if } \\
\text { this assessment is confirmed } \\
\text { that the common people might } \\
\text { ask who serves as their } \\
\text { protective umbrella. Secretary } \\
\text { Cheng, you and I have both } \\
\text { been transterred here recently. } \\
\text { May that not cause } \\
\text { misunderstandings? } \\
\text { Cheng Zhl: Actually, we don't } \\
\text { have to worry about this. The } \\
\text { provincial government called } \\
\text { me in first and then called in } \\
\text { you in order to full-heartedly } \\
\text { change the face of Yundu's } \\
\text { public security. (...) }\end{array}$ & $\begin{array}{l}\text { The provincial } \\
\text { leadership supports } \\
\text { the law enforcement } \\
\text { efforts. } \\
\text { - The provincial and } \\
\text { police leadership have } \\
\text { the responsibility to } \\
\text { protect the people. } \\
\text { The two leaders are } \\
\text { professionals who } \\
\text { have been calied upon } \\
\text { to reform law } \\
\text { enforcement in the } \\
\text { city. }\end{array}$ \\
\hline $\begin{array}{l}\text { GAJZ 10-10: } \\
\text { Li Jian reports } \\
\text { back to Cheng } \\
\text { Zhi on a bribery } \\
\text { attempl by } \\
\text { crime-lord Ma } \\
\text { Fugui, and } \\
\text { explains his } \\
\text { strategy to fight } \\
\text { crime. }\end{array}$ & 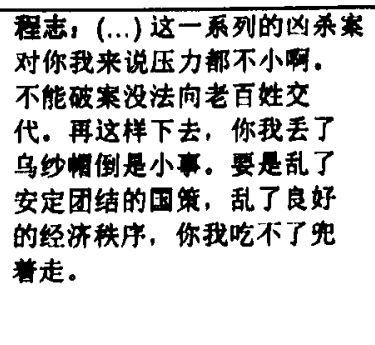 & $\begin{array}{l}\text { Cheng Zhi: (...) This series of } \\
\text { murder cases is putting a lot of } \\
\text { pressure on both of us. If we } \\
\text { can't solve these cases, we } \\
\text { can't justify ourselves to the } \\
\text { common people. If this } \\
\text { continues, you and I losing our } \\
\text { posts will be the smallest of } \\
\text { our worries. If the national } \\
\text { policies for stability and unity } \\
\text { as well as the good economic }\end{array}$ & $\begin{array}{l}\text { The provincial and } \\
\text { police leadership } \\
\text { answer to the people. } \\
\text { The provincial and } \\
\text { police leadership have } \\
\text { the responsibility to } \\
\text { protect social stability } \\
\text { and economic } \\
\text { development (which } \\
\text { are threatened by }\end{array}$ \\
\hline
\end{tabular}




\begin{tabular}{|c|c|c|c|}
\hline & 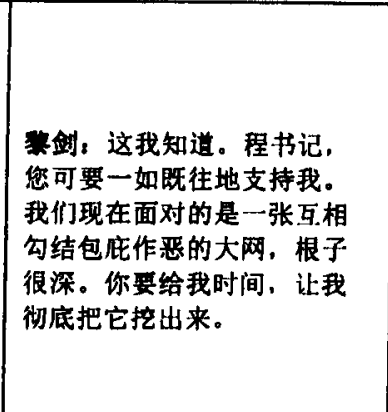 & $\begin{array}{l}\text { arrangements fall into disarray, } \\
\text { then you and I will be in } \\
\text { serious trouble. } \\
\text { LI Jian: I know. Secretary } \\
\text { Cheng, you may have to back } \\
\text { me up again. We are currently } \\
\text { faced with a web that is } \\
\text { covering up a collaboration of } \\
\text { evildoers. The roots are deep. } \\
\text { You have to give me time so } \\
\text { that I can thoroughly dig them } \\
\text { up. }\end{array}$ & $\begin{array}{l}\text { crime). } \\
\text { The provincial } \\
\text { leadership supports } \\
\text { the law enforcement } \\
\text { efforts. }\end{array}$ \\
\hline $\begin{array}{l}\text { GAJZ 14-14: } \\
\text { Cheng Zhi } \\
\text { invites Li Jian } \\
\text { for lunch and a } \\
\text { drink after a } \\
\text { failed murder } \\
\text { attempt on Li's } \\
\text { life. }\end{array}$ & $\begin{array}{l}\text { 程志: 对你前一段的工作我 } \\
\text { 和者厅长非常满意。 }\end{array}$ & $\begin{array}{l}\text { Cheng Zhl: Director Cao and I } \\
\text { are very pleased with your } \\
\text { work so far. }\end{array}$ & $\begin{array}{l}\text { The provincial } \\
\text { leadership oversees } \\
\text { the law enforcement } \\
\text { effort and is satisfied } \\
\text { with the } \\
\text { commissioner's } \\
\text { progress. }\end{array}$ \\
\hline $\begin{array}{l}\text { GAJZ 14-14: } \\
\text { Cheng Zhi } \\
\text { invites Li Jian } \\
\text { for lunch and a } \\
\text { drink after a } \\
\text { failed murder } \\
\text { attempt on Li's } \\
\text { life. }\end{array}$ & 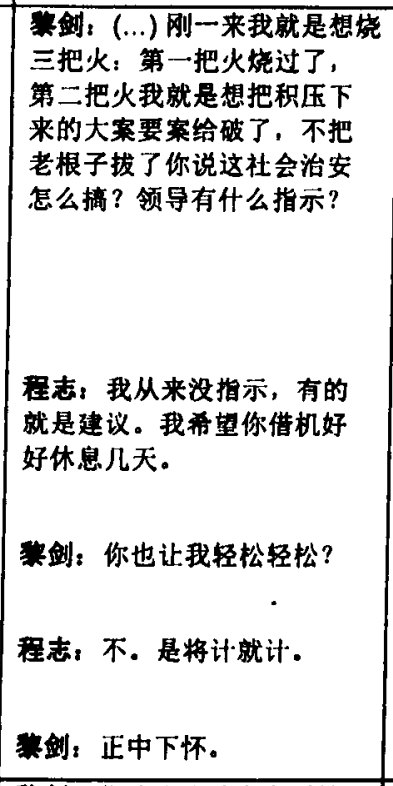 & $\begin{array}{l}\text { LI Jian: (...) When I had just } \\
\text { arrived, I wanted to attend to } \\
\text { three fires: the first fire I have } \\
\text { tended to, the second fire was } \\
\text { to solve the connected cases } \\
\text { that had been put off before. If } \\
\text { we don't pull out the roots, } \\
\text { how can we uphold the public } \\
\text { order? What are the } \\
\text { leadership's instructions? } \\
\text { Cheng Zhl: I never had any } \\
\text { instructions, I only have } \\
\text { suggestions. I hope you'll take } \\
\text { the opportunity to rest for a } \\
\text { few days. } \\
\text { Ll Jian: You also want me to } \\
\text { take it easy? } \\
\text { Cheng Zhl: No, it's to beat } \\
\text { them at their own game. } \\
\text { LI Jlan: That's exactly what I } \\
\text { was hoping for. }\end{array}$ & $\begin{array}{l}\text { The provincial } \\
\text { leadership potentially } \\
\text { has the power to } \\
\text { decree how the law } \\
\text { should be enforced, } \\
\text { but chooses instead to } \\
\text { merely advise the } \\
\text { police. } \\
\text { The provincial } \\
\text { leadership } \\
\text { understands the } \\
\text { strategies necessary } \\
\text { to fight crime. }\end{array}$ \\
\hline $\begin{array}{l}\text { GAJZ 16-02: } \\
\text { Cheng Zhi visits } \\
\text { Li Jian's office } \\
\text { to talk about the } \\
\text { corruption } \\
\text { accusations } \\
\text { against Li's } \\
\text { deputy Huo } \\
\text { Xiang. }\end{array}$ & 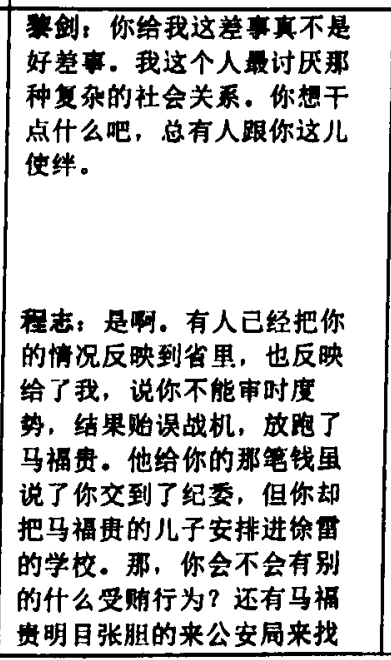 & $\begin{array}{l}\text { LI Jlan: This commission you } \\
\text { gave me really isn't a good } \\
\text { commission. The things I } \\
\text { loathe most are these types of } \\
\text { complicated social relations. } \\
\text { Every time you try to get } \\
\text { something done, there's } \\
\text { always someone who is trying } \\
\text { to undermine you. } \\
\text { Cheng Zhl: Yeah. Some have } \\
\text { already reported your situation } \\
\text { to the province, and have } \\
\text { reported it to me, saying you're } \\
\text { not impartial, and that this } \\
\text { hinders police (military) } \\
\text { operations; that that's why you } \\
\text { let Ma Fugui escape. That } \\
\text { money he gave you, even } \\
\text { though you handed it over to } \\
\text { the disciplinary commission, }\end{array}$ & $\begin{array}{l}\text { - Law enforcement is } \\
\text { hampered by complex } \\
\text { social relations, which } \\
\text { are on occasion } \\
\text { interpreted as } \\
\text { corruption. } \\
\text { - Law enforcement is } \\
\text { under public scrutiny } \\
\text { for corruption. } \\
\text { The provincial } \\
\text { leadership monitors } \\
\text { the law enforcement } \\
\text { efforts and } \\
\text { investigates corruption } \\
\text { accusations. } \\
\text { The police } \\
\text { commissioner is } \\
\text { responsible for the }\end{array}$ \\
\hline
\end{tabular}




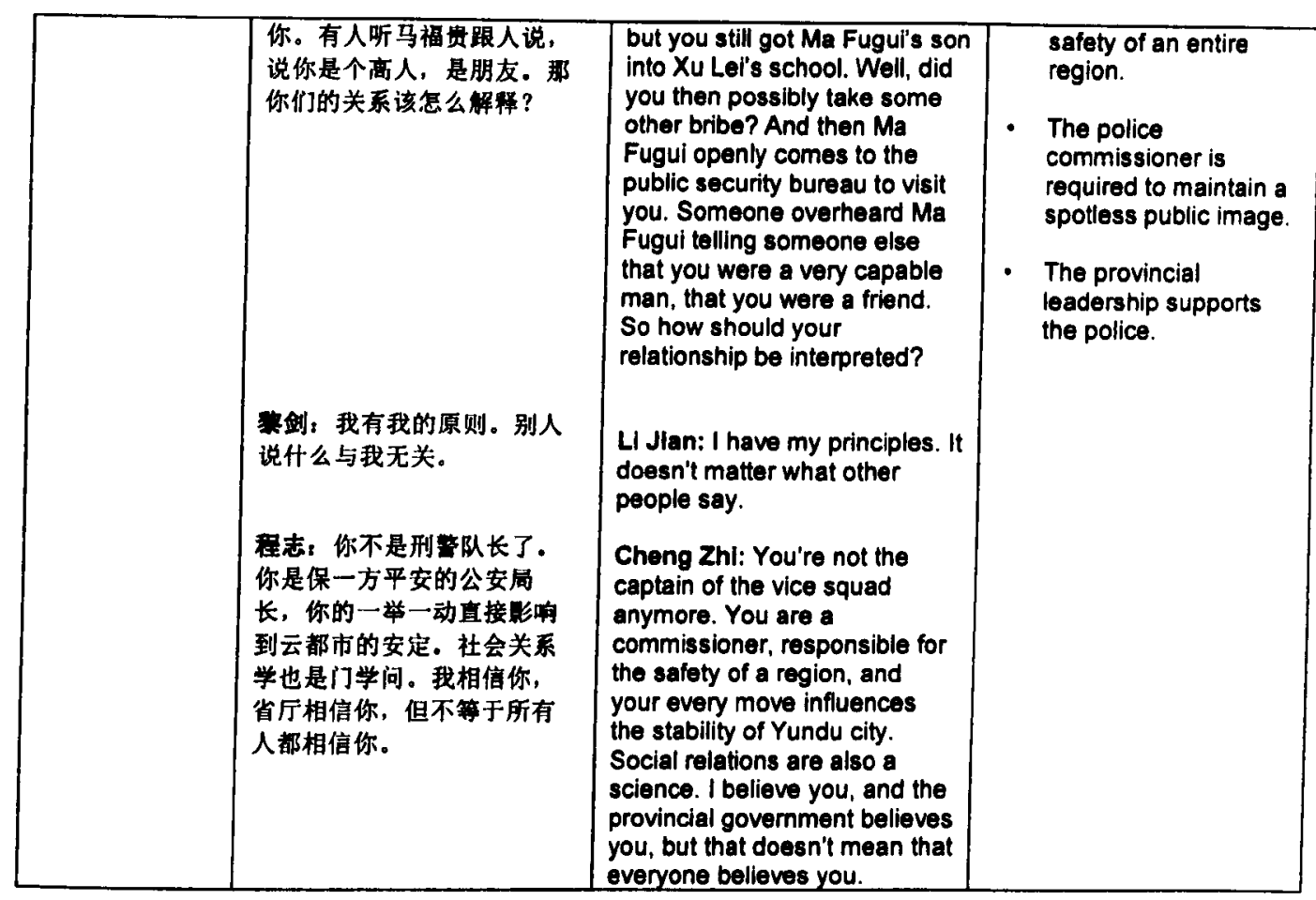

To summarise these findings, the provincial leadership and the police force in GAJZ work as a team. The leadership is portrayed as a responsible, professional administration that oversees police work, investigates corruption within the police force and facilitates reform. At the same time, the leadership understands the difficulties that police officers are faced with when fighting crime: it grants the law enforcement experts a high degree of autonomy, trusts their judgement and supports their efforts full-heartedly. Moreover, both the provincial and the law enforcement leadership serve and answer to the people.

The concept that the party and state serve the people (in line with the Mao Zedong quote wei renmin fuwu 为人民服务) features strongly in GAJZ, and is particularly dominant in the sequences that deal with those being governed, i.e. the people. In order to demonstrate this, I shall turn to a narrative arc in episode 8 that focuses not on Cheng Zhi, but instead on Commissioner $\mathrm{Li}$ and his police force. The arc consists of three sequence: one in which Li Jian gives a speech to the police force on the meaning of being an officer (GAJZ 08-05), a sequence 
during which the commissioner inspects a female police official doing her job (GAJZ 08-07), and finally a sequence of police officers around the city serving the people (GAJZ 08-08).

First, the sequence during which $\mathrm{Li}$ Jian addresses the police force is again a scene that relies heavily on a verbal message. Nonetheless, a number of visual and acoustic signs are worth mentioning: the general arrangement of the characters and objects in the scene places Commissioner Li behind a podium, along with his deputies Huo Xiang and Dai Wenqing, as well as Secretary Cheng. The audience (i.e. the police force) are seated on blue chairs, and the montage of shots suggests they are facing the podium. Behind the podium, a red curtain functions as the backdrop, and a red banner with white characters reads: "mobilization meeting for the strengthening of troop construction, the management of the police by law, and the strict management of the police" (jiaqiang duiwu jianshe, yi fa zhi jing, congyan zhi jing dongyuan dahui 加强队伍建设, 以法治警, 从严治警动员大会). The red (i.e. national colour) theme here emphasises the gravity and official nature of the meeting.

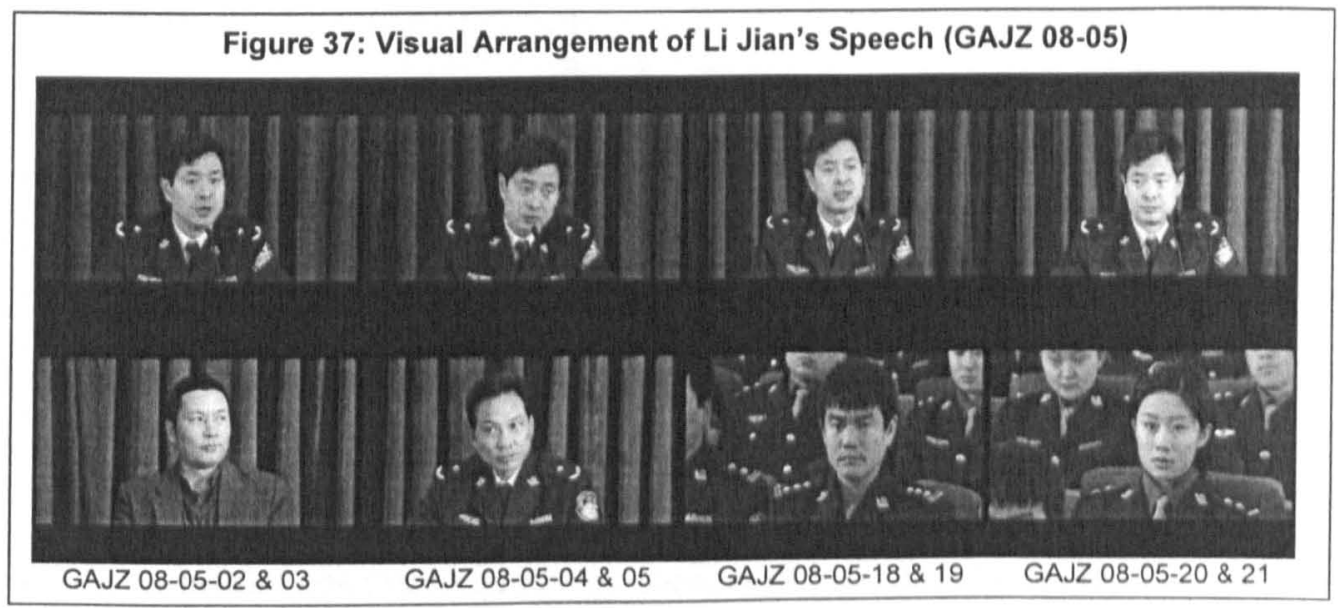

The sequence opens with a close-up of the propaganda banner, the camera panning slowly to the right to reveal the entire slogan (this is the only camera movement in the sequence, aside from a zoom in shot 16). After this initial close-up, the sequence then alternates between lengthy medium close-up shots of Li Jian giving his speech and quick shots of his listeners' reaction, i.e. medium close-ups of Cheng Zhi, Huo Xiang, and Dai Wenqing sitting at the 
podium, as well as of Officers Chen Xiaorong 陈晓荣, Zhang 张, Liu Qiong 刘琼, Wu 吴, Lin Jun 林军, and an unnamed officer sitting in the audience. The ways in which these characters are placed in the scene as well as their demeanour suggest support for Li Jian from those seated at the podium and agreement to his words on the side of the audience (Figure 37). In the middle of the sequence, two very lengthy long shots show the entire podium, including the propaganda banner, as Li continues his speech (shots 14 and 16). The shot frequency increases during the last few shots of the sequence, as Li concludes his speech and his audience applauds enthusiastically (see Figure 38). With the exception of the first shot, the entire sequence is underscored by a sentimental trumpet melody (the shot of the propaganda banner is underscored by drums).

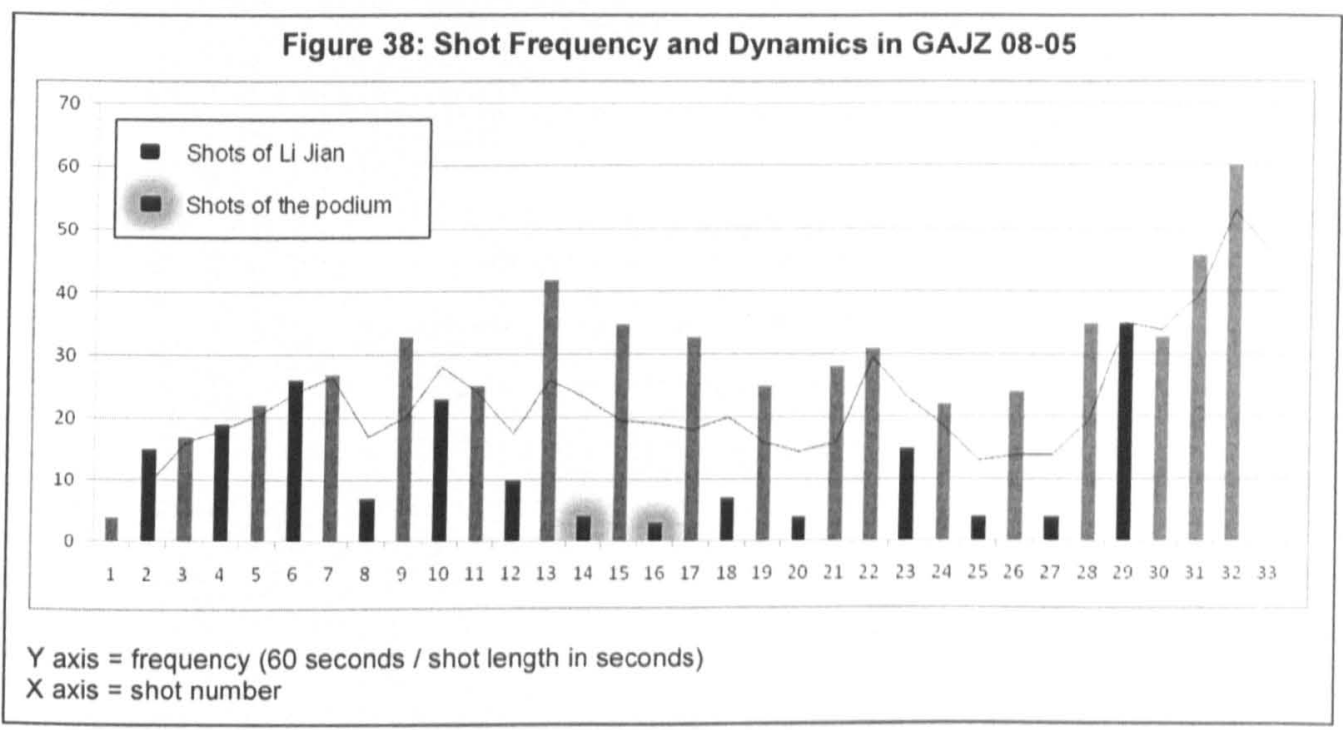

Throughout this sequence, Li Jian is giving a speech in which he admonishes bad behaviour within the police force. I have divided the speech into units (based on punctuation and shot division), and have paraphrased the main arguments made in each unit (see Figure 39).

Figure 39: Li Jian's Speech on the Conduct of Good Police Officers (GAJZ 08-05)

\begin{tabular}{|c|c|c|c|}
\hline $\begin{array}{l}\text { Shot } \\
\text { No. }\end{array}$ & $\begin{array}{l}\text { Chinese } \\
\text { Original }\end{array}$ & $\begin{array}{l}\text { English } \\
\text { Translation }\end{array}$ & $\begin{array}{l}\text { Main Argument and Rhetoric } \\
\text { Devices (in brackets) }\end{array}$ \\
\hline 1 & $\begin{array}{l}\text { 黎剑: "你们不为自 } \\
\text { 己的行为感到可笑。 } \\
\text { 治安先治撆, 治整先 } \\
\text { 治长。 }\end{array}$ & $\begin{array}{l}\text { Li Jian: "You don't think your conduct is } \\
\text { ridiculous. In order to ensure public } \\
\text { security, we need to first manage the } \\
\text { police force; in order to manage the }\end{array}$ & $\begin{array}{l}\text { - Public security depends on a } \\
\text { well-managed police force, } \\
\text { which in turn requires a well- } \\
\text { managed leadership }\end{array}$ \\
\hline
\end{tabular}




\begin{tabular}{|c|c|c|c|}
\hline & & $\begin{array}{l}\text { police force, we need to first manage the } \\
\text { leadership. }\end{array}$ & $\begin{array}{l}\text { (rhetorical question; } \\
\text { parallelism \& climax). }\end{array}$ \\
\hline $2-4$ & $\begin{array}{l}\text { 人民是父母。服务群 } \\
\text { 众是我们的天职。我 } \\
\text { 们的民整大多数是尽 } \\
\text { 职的. }\end{array}$ & $\begin{array}{l}\text { The people are our parents. To serve the } \\
\text { masses is our bounded duty. The } \\
\text { majority of our people's police officers } \\
\text { fulfil their duty. }\end{array}$ & $\begin{array}{l}\text { - The police serve the people } \\
\text { (reversed paternalistic } \\
\text { metaphor). } \\
\text { - The police generally fulfil their } \\
\text { duty (generalization) }\end{array}$ \\
\hline $5-8$ & 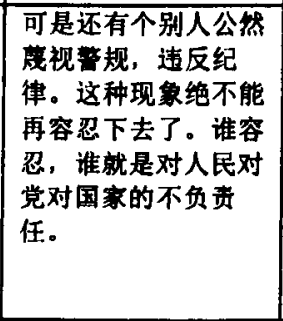 & $\begin{array}{l}\text { However, there are a few individuals who } \\
\text { openly ignore police guidelines and } \\
\text { violate the laws and regulations. Such } \\
\text { phenomena shall never again be } \\
\text { tolerated. Anyone who does tolerate } \\
\text { them is being irresponsible towards the } \\
\text { people, the party, and the nation. }\end{array}$ & $\begin{array}{l}\text { - There are some bad police } \\
\text { officers (indefinite pronoun } \\
\text { generalization). } \\
\text { Bad conduct within the police } \\
\text { force will not be tolerated } \\
\text { (hyperbole; metaphor: human } \\
\text { behaviour as phenomenon). } \\
\text { The police answer to the } \\
\text { people, the party, and the } \\
\text { nation (tri-colon). }\end{array}$ \\
\hline $9-11$ & $\begin{array}{l}\text { 我们人民部囬的宗旨 } \\
\text { 是什么？是为人民服 } \\
\text { 务. }\end{array}$ & $\begin{array}{l}\text { What is the purpose of the people's } \\
\text { police? It is to serve the people. }\end{array}$ & $\begin{array}{l}\text { The police serve the people } \\
\text { (hypophora). }\end{array}$ \\
\hline 12 & $\begin{array}{l}\text { 可是我们有的人却戏 } \\
\text { 言人民整察就是抓小 } \\
\text { 偷。” }\end{array}$ & $\begin{array}{l}\text { Yet some people joke and say that the } \\
\text { people's police are about arresting petty } \\
\text { thieves." }\end{array}$ & $\begin{array}{l}\text { - The police do more than } \\
\text { arrest thieves (indefinite } \\
\text { pronoun generalization; joke: } \\
\text { synecdoche \& trivialization). }\end{array}$ \\
\hline 13 & 翌察: [笑] & Police Officers: [laugh] & \\
\hline 14 & $\begin{array}{l}\text { 繁剑: “你们不要 } \\
\text { 笑.这何戏吉乍听起 } \\
\text { 来没什么。可是这何 } \\
\text { 话用在了自己失职做 } \\
\text { 辩解的时候让人听起 } \\
\text { 来就脸红。 }\end{array}$ & $\begin{array}{l}\text { Li Jiang: "You shouldn't laugh. This } \\
\text { sentence at first doesn't seem wrong. But } \\
\text { if it is used to justify misconduct, it will } \\
\text { make anyone who hears it blush with } \\
\text { shame. }\end{array}$ & $\begin{array}{l}\text { - The crime-fighting function of } \\
\text { the police should not be used } \\
\text { to conceal misconduct } \\
\text { (appeal: to sense of honour; } \\
\text { imagery). }\end{array}$ \\
\hline $16-19$ & 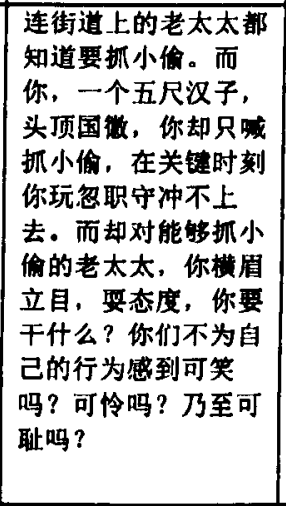 & $\begin{array}{l}\text { All the old ladies in the street know that } \\
\text { we arrest pelty thieves. And yet you, a } \\
\text { five foot tall fellows with the nation's } \\
\text { emblem on your hat, you're still yelling } \\
\text { out that your arresting pelty thieves... in a } \\
\text { moment of truth such dereliction of duty } \\
\text { will not hold up. However you scom at an } \\
\text { old lady who is able to arrest a petty thief. } \\
\text { frowning as you file her case, you lose } \\
\text { your temper, what is that about? Don't } \\
\text { you think your conduct is ridiculous? } \\
\text { Pitiful? Even shameful? }\end{array}$ & $\begin{array}{l}\text { The police's crime fighting } \\
\text { function is commonly known } \\
\text { (synecdoche \& trivialization; } \\
\text { imagery). } \\
\text { Covering up derelictions of } \\
\text { duty by referring to that } \\
\text { function will not go unnoticed } \\
\text { (synecdoche \& trivialization; } \\
\text { appeal: to patriotic sentiment; } \\
\text { imagery; trope: moment of } \\
\text { truth). } \\
\text { It is unacceptable for an } \\
\text { officer to look down on the } \\
\text { people (synecdoche \& } \\
\text { trivialization; tri-colon } \\
\text { rhetorical question; climax) }\end{array}$ \\
\hline 20 & 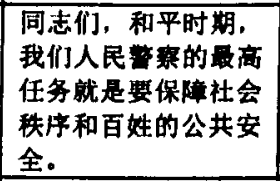 & $\begin{array}{l}\text { Comrades, in peace times, the main task } \\
\text { of the people's police is to safeguard the } \\
\text { social order and the public safety of the } \\
\text { common people. }\end{array}$ & $\begin{array}{l}\text { The police's function in peace } \\
\text { times: uphold the social order } \\
\text { and protect the people } \\
\text { (personal address: comrades; } \\
\text { definition). }\end{array}$ \\
\hline $21-24$ & 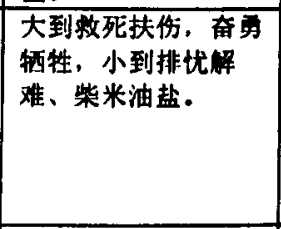 & $\begin{array}{l}\text { In the extreme this may mean that we } \\
\text { have to rescue the dying and heal the } \\
\text { wounded, muster our courage and } \\
\text { sacrifice ourselves. At the very least it } \\
\text { means that we have to overcome } \\
\text { difficulties and provide for the daily } \\
\text { necessities. }\end{array}$ & $\begin{array}{l}\text { Police work may involve } \\
\text { extreme sacrifices (hyperbole; } \\
\text { parallelism; definition). } \\
\text { Police work generally means } \\
\text { providing for the people } \\
\text { (parallelism; definition). }\end{array}$ \\
\hline 25 & 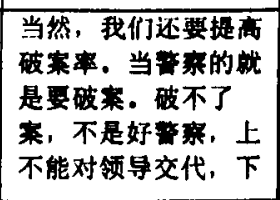 & $\begin{array}{l}\text { Of course. we also have to increase the } \\
\text { rate at which we solve crime cases. Of } \\
\text { course, police officers have to solve } \\
\text { crime cases. If you can't solve the case, } \\
\text { you are not a good police officer. You } \\
\text { won't be able to justify yourself towards }\end{array}$ & $\begin{array}{l}\text { - Fighting crime is an important } \\
\text { part of police work (anaphora; } \\
\text { procatalepsis). } \\
\text { - If the police do not fulfil that } \\
\text { function, they lose their public } \\
\text { mandate (personal address). }\end{array}$ \\
\hline
\end{tabular}




\begin{tabular}{|c|c|c|c|}
\hline & 不能对百姓交代。 & $\begin{array}{l}\text { the leadership above or the common } \\
\text { people below. }\end{array}$ & $\begin{array}{l}\text { The police answer to the } \\
\text { people and the leadership } \\
\text { (parallelism; allegory). }\end{array}$ \\
\hline $26-27$ & $\begin{array}{l}\text { 所以我在这里头郑重 } \\
\text { 承诺公安部、省厅督 } \\
\text { 办的 } 8 / 26 \text { 等大案要 } \\
\text { 案, 一年内破不了我 } \\
\text { 首先引各辞职。" }\end{array}$ & $\begin{array}{l}\text { Therefore I declare here in all } \\
\text { seriousness to the Ministry of Public } \\
\text { Security and the provincial government } \\
\text { who are overseeing our work on cases } \\
\text { such as the August } 26 \text { th incident, that if } \\
\text { we are unable to solve these major cases } \\
\text { within one year, I will be the first to take } \\
\text { the blame and resign. }\end{array}$ & $\begin{array}{l}\text { The Ministry of Public } \\
\text { Security and the provincial } \\
\text { government oversee the } \\
\text { police's law enforcement } \\
\text { efforts. } \\
\text { - The commissioner is } \\
\text { personally responsible for the } \\
\text { police's performance (pledge) }\end{array}$ \\
\hline
\end{tabular}

The verbal rhetoric techniques as well as the visual and acoustic signs adopted in this sequence function together to make the following discursive statements (which at this point cross the discourse on governing with that on public security):

- The police and their leadership serve the people, and answer to the people.

- A public leader is personally responsible for his subordinates' performance.

- Police work is under public and governmental scrutiny.

- Public service is a patriotic duty.

Particularly the idea that law enforcement is equivalent to public service is then picked up

and reiterated in the other two sequences of this narrative arc. In the first of these sequences

(GAJZ 08-07), this point is exemplified through the work of a female police officer.

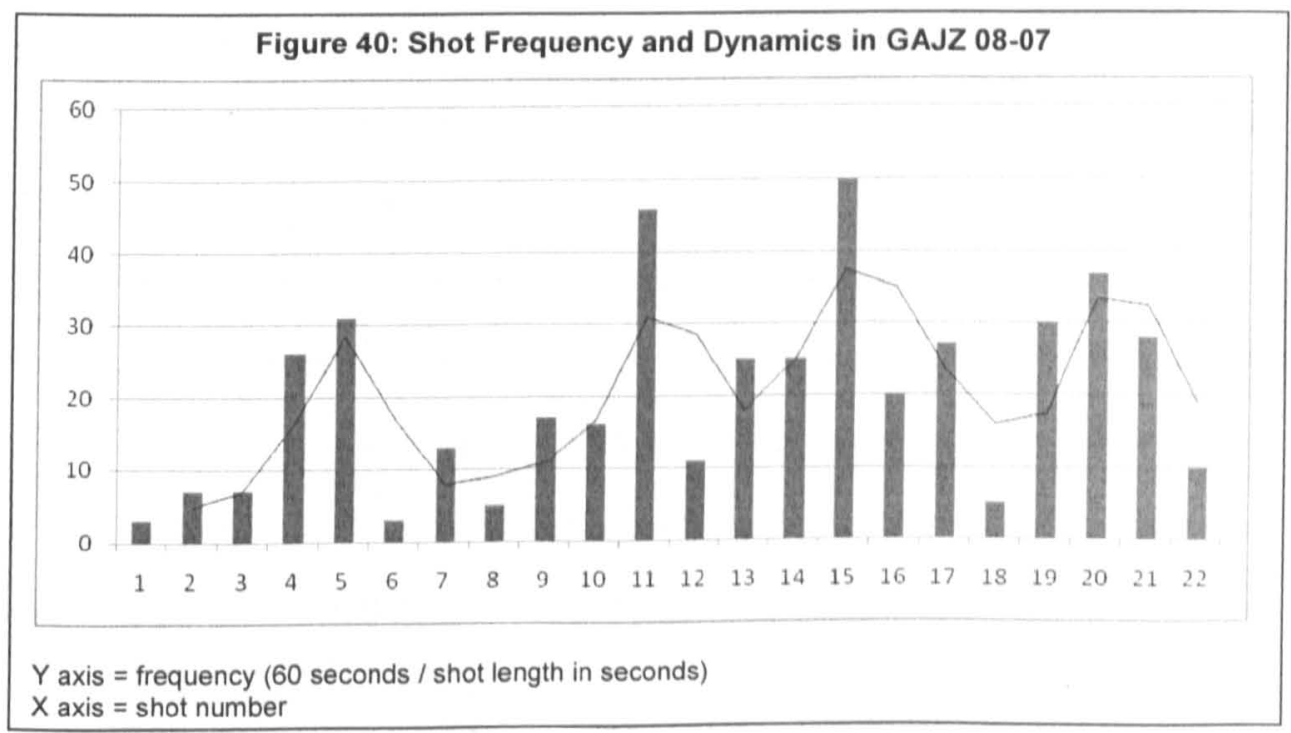


The sequence, which consists of one scene, is set in the service area of the police precinct. It starts with the longest shot of the sequence: an establishing shot that first shows a filing case (close-up), and then zooms out to reveal the female officer filing documents behind the service desk (medium shot). A woman carrying a baby arrives at the desk and asks for help with her child's residence permit, apologizing for arriving after hours. The rest of the scene is largely arranged as shots aimed through the window of the service desk, from either side of the counter. The dynamics divide the scene into four segments (see Figure 40): during the first six shots, the female officer handles the women's case; second, Li Jian arrives and asks the officer a professional question (shots 07 - 11); during the third section, Li asks another professional question as well as the officer's name (shots 12 - 18); and finally, Li Jian commends the female officer for her good work and leaves (shots 18 - 22).

The first section is used to make the statement: The female officer is polite, helpful, dedicated, and efficient (see Figure 41). This is achieved through the juxtaposition of shots showing the officer working (particularly a close-up showing her hands typing on a computer keyboard; shot 04) and the person she is helping out (particularly the close-up of the woman's baby; shot 05). Acoustic signs are used on the one hand to clarify that the help desk is a public space (cars honking, traffic noise), and on the other to emphasise that the officer is being industrious (keyboard typing). The editing in this part highlights that the officer is a fast worker and that applying for a residence permit is a minor formality (the officer needs roughly twenty seconds to complete the task). The verbal signs further underline two discursive statements: first, that the officer is dedicated to her work (the woman stating that she is late in shot 01 , and the officer nonetheless helping out without much further ado); second that the officer is efficient at her job (shot 06): she presents the final documents with a simple: "There" (gei 给), and the woman then gasps in surprise: "Done? And so fast! Thank you, thank you!" (Haola? Zheme kuai! Xiexie, xiexie ah! 好啦? 这么快! 谢谢, 谢谢啊!). At the 
same time, another visual sign is used to emphasise that the officer is working late: Li Jian arrives in the background of shot 06 , looks at the help desk, and then checks his watch.

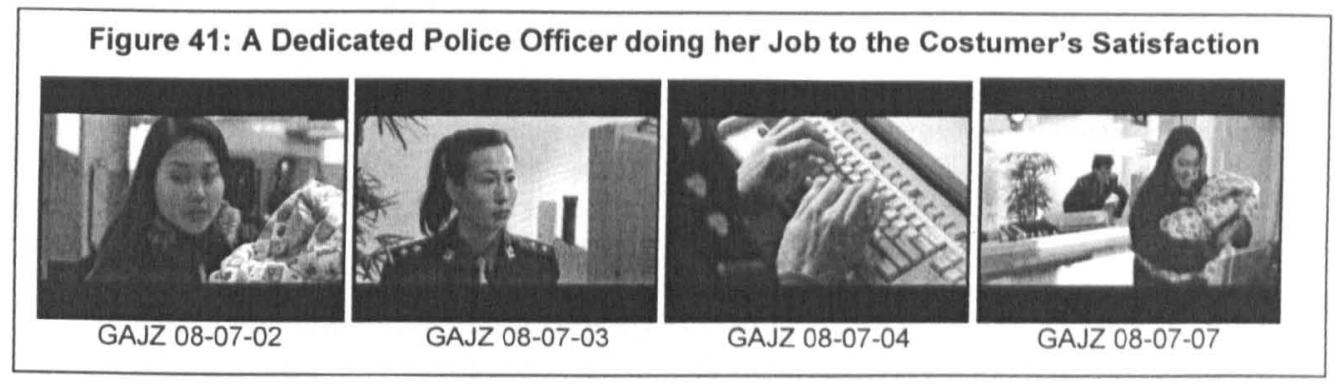

As the scene continues, the woman with her child walks off, smiling happily, and Li Jian

takes a seat at the help desk (shot 07). After reinforcing verbally that it is after hours (Li:

“you're still working this late?"; zheme wanle hai mei xiaban? 这么晚了还没下班? ), Li

introduces himself, and inquires whether he may quiz the officer about her work (shot 08).

He then first asks: "what do police officers working at the service desk need to accomplish?"

(chuanghu danwei jiedai minjing yao zuo dao nar dian? 窗户单位接待民警要做到哪几点? shot 08)

and then: "what documents do persons require who have given birth to a child abroad and

wish to receive a residence permit for the child upon their return" (chuguo renyuan zai guowai

shenyu de zinü huiguo luo hukou xuyao chi naxie zhengjian? 出国人员在国外生育的子女同国落户

口需要持哪些证件? shot 12). During these two sections, the shots of the female officer

undergo a change in perspective, and she is now continuously shown in frontal brief

medium close-ups (emphasizing the interview character of the conversation; shots 09, 11, 13,

15, 17, 19 and 21). Her verbal answers to the two questions are (shots $09-10$ and $13-15$ ):

Officer $\mathrm{Xu}$ : Their manners should be dignified, their appearance neat and professional, their attitude enthusiastic and their language civilised.

许警察: 举止端庄, 警容严整, 态度热情语言文明。

Officer $\mathrm{Xu}$ : The birth certificate of the child that was born abroad, the parents' passports, and the passport that the child used to return to the country.

许警察：国外出生小孩的出生证明、父母的护照、小孩回国使用的护照。 
These answers are offered without hesitation, and their brevity and clarity again underline the efficiency of the officer. That these answers are satisfying is made clear by various visual signs (Li Jian looking at his colleagues Dai Wenqing and Fang Hua 方华, an impressed look on his face; shot 10) and additional verbal reinforcement (Li praising the officer's diligence and expertise; shot 18). Finally (shot 18-20), Li Jian sums up the encounter by stating:

The establishment of our image project is not just a pretence that we enact for others but is meant to establish an ideology of wholeheartedly serving the people. Wouldn't you agree?

我们树形象工程不是做样子演给人家看而是要树立全心全意为人民服务的思 想。你说我说得对吗?

The female officer then enthusiastically agrees with Li's assessment (shot 21), and they shake hands through the service window before $\mathrm{Li}$ moves on to attend a meeting (shot 22).

All in all, this sequence can then be said to make the following discursive statements:

- Law enforcement is a service provided by the police to the people.

- Good public servants are polite, helpful, dedicated, diligent, professional, efficient and well-spoken.

- Public leaders care personally about the good performance of their subordinates.

Again, the main emphasis is placed on the state serving the people. The extent to which the police help the common people is then demonstrated visually in the final sequence of this narrative arc (GAJZ 08-08). Here, an arrangement of scenes shows police officers at work. Three different types of police activity are depicted:

First, an extremely long shot shows two officers walking through a neighbourhood, greeting people on the street and shaking hands with the local inhabitants. The same two officers are then shown having a conversation with an old lady, asking about her wellbeing and assuring her that she can come by the precinct anytime she needs help (shot 02). A similar situation is later depicted in shot 06 , with the two officers again chatting to an old lady. 
Second, a three-shot scene shows a police officer regulating traffic. The female officer is shown at a low angle as she waves traffic through (shot 03), and then at a distance (long shot) as the traffic roles by in front of her (shot 04). Finally, the last shot pans left as it follows a car driving by, establishing visually that the traffic is indeed flowing (shot 05).

Third, another female traffic warden (possibly the same as above) is shown helping a blind man across the street. The man is thanking her as they walk across a pedestrian crossing (shot 07).

The sequence then ends with two police officers (possibly the same as in shots 01,02 and 06) receiving a reward at the local police station from the community for their sociable behaviour. In this sense, the above activities are positively sanctioned by 'the people': In shot 08, the camera pans right as Commissioner Li motions the community representatives into a room filled with applauding police officers. The two officers who receive the reward are standing at the far side, before a red curtain, and below the national police emblem. A red banner reads: "carry out profound activities for the establishment of service mechanisms" (shenru kaizhan jianshe fuwuxing jiguan huodong 深入开展建设服务型机关活动). The civilians hand a red flag with golden calligraphy to the two officers (the characters on the flag are illegible at this angle). The final shot of the sequence then shows the community representatives leaving the police stations past two rows of clapping police officers, while Commissioner $\mathrm{Li}$ and others see the guests off (waving and applauding). Another banner is shown in the background, reading: "actively participate in the annual grassroots activities for competent citizens" (jiji canjia jicheng zhan suo xing min jianshe nian huodong 积极参加! 基/2? 站所行民建设年活动). The entire sequence is underscored with a dynamic military march. A number of discursive statements are made here, most of which are related to the public security discourse, and concerning the responsibilities of the police: 
- The police are helpful, sociable, and part of the community.

- The police ensure the flow of traffic.

- The police help those in need, even with minor tasks.

- The people appreciate the police force's efforts.

What is important with regard to the discourse on governance is that the sequence visually reinforces the discursive statement:

- Public servants serve the people.

It is particularly noteworthy who 'the people' in this narrative arc are. In the two sequences GAJZ 08-07 and GAJZ 08-08, the police help the following 'common people': a mother, a little baby, old ladies and an old blind man (see Figure 42). In this sense, there seems to be a parallel to the people depicted in HWDD: in both dramas, the 'common masses' consist of children as well as elderly, largely female, and possibly disabled persons.

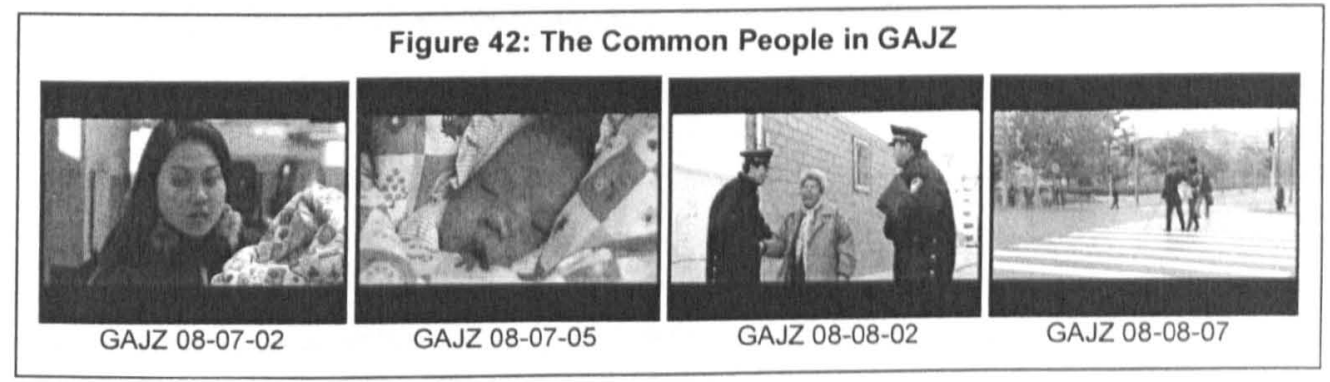

\section{2.ii The Military Nature of Public Security}

Some discursive statements concerning public security have already been made above, in connection with the governance discourse. In general, law enforcement is shown as a service that the police provide to the people. What is noteworthy about the security discourse in GAJZ is that law enforcement is in addition equated with military activity. In sequences where law enforcement means more than community service, the fight against crime is visually, acoustically and verbally arranged through signs that are borrowed heavily from war discourses. The analysis of two sequences shall serve to exemplify this. 
The first of these sequences has already been mentioned: it is the hostage situation during which Secretary Cheng Zhi makes his debut appearance (GAJZ 01-11). A key aspect of this sequence is the use of images that feature weapons and heavy armour. Almost half of the shots in this sequence show law enforcement officials carrying, loading or aiming some form of firearm (Figure 43).

In some cases the firearms are a subtle background aspect of the shots, in others they are the dominant feature (Figure 44). At any rate, images of weapons in the hands of police officers seem ever present. The fixation on weaponry is particularly obvious when police officers load their shotguns (for instance shots 16, 18 and 23); a gesture that signifies readiness for battle, but in some cases has no actual relevance to the plot (see particularly shots 50 and 93 , in which the same police officers load their guns twice, as well as shot 26 , in which a row of officers performs the characteristic pump-action movement in synchronicity). Other types of firearm images include close-ups of shotgun barrels (shots 48 and 60 ), medium close-ups as well as medium shots of snipers aiming their rifles from behind cover (shots 49, 52, 79, 81, 83, 97, 112 and 132), shots that show Special Forces squads in green camouflage (!) uniforms armed with automatic rifles (shots 10-14, 19-21, 24, 27, 34, 43-45, 80, 102, 105, 107, 109, 113 and 120), and regular police officers readying their side-arms (shots $04-06$ ). Note also how shots of the main protagonists in this scene (i.e. Huo Xiang and Cheng Zhi) are arranged in a way that almost always places heavy weaponry in the frame as well (three perspectives are relevant in this regard: the one shown in shots $35,36,37,40,41,43,44,45$ and 46 ; the one in shots 56, 65, 72, 74 and 76; as well as the one in shots 50, 93, 106, 111 and 115). 


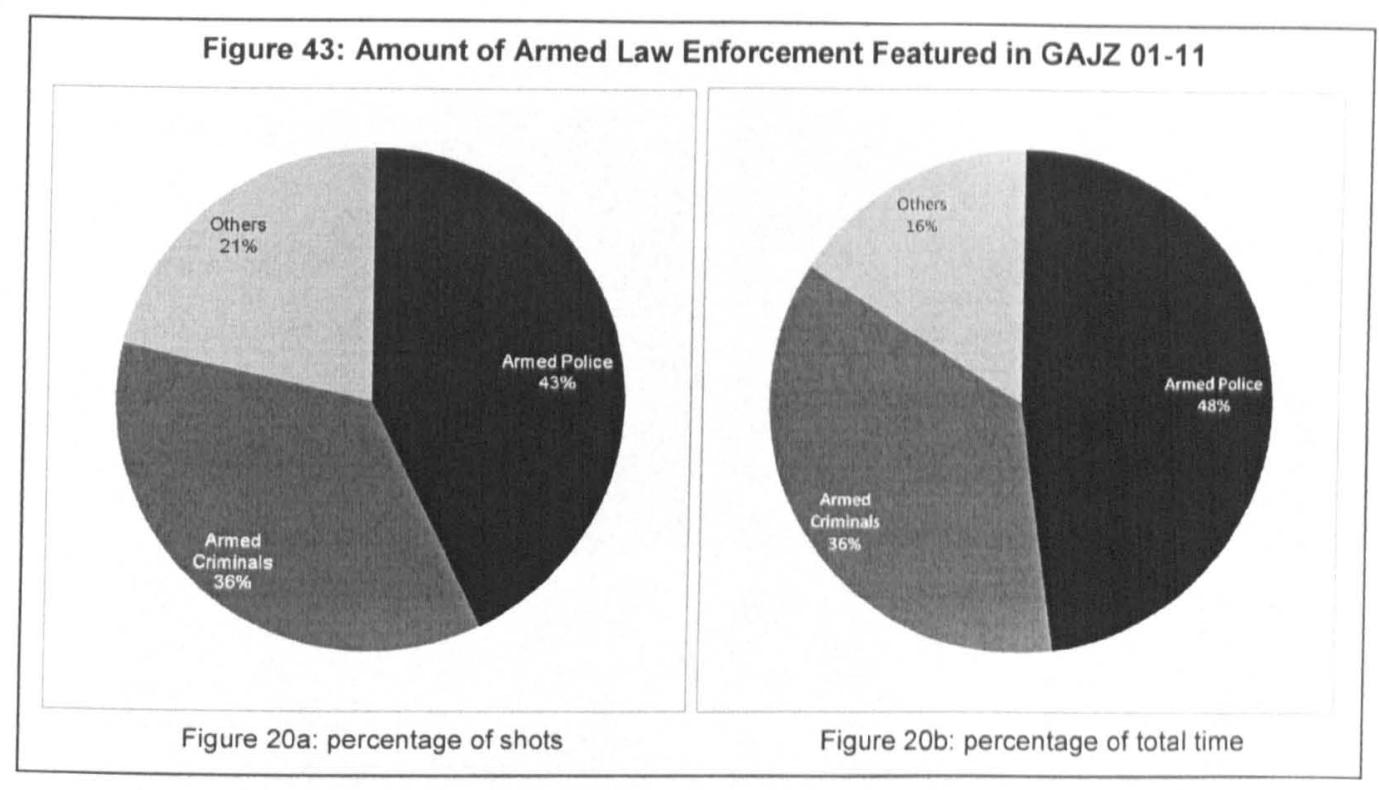

Considering that the music used in the sequence also possesses a distinct military quality, and that the shots of firearms are edited at a high pace to increase tension, it can be said that the visual and acoustic signs in this sequence are tailored to inform the viewers that they are witnessing a military operation, and to hence make the discursive statement:

- Law enforcement is warfare.

The degree to which weaponry is shown here stands in rather stark contrast on one hand to the threat that the police force actually faces (two criminals carrying pistols, with one of the men also being armed with a suicide bomb) and on the other hand to the way the situation is later resolved: one criminal is shot in the back of the head at close range with a handgun (implied in shot 131), the other is thrown off of the ship's railway (shots 155-158) where he explodes (implied in shot 159 by a fade to white and the sound of an explosion). None of the firepower brought to bear on the two rather unprofessional criminals in this sequence actually comes into play at any point of the operation. 


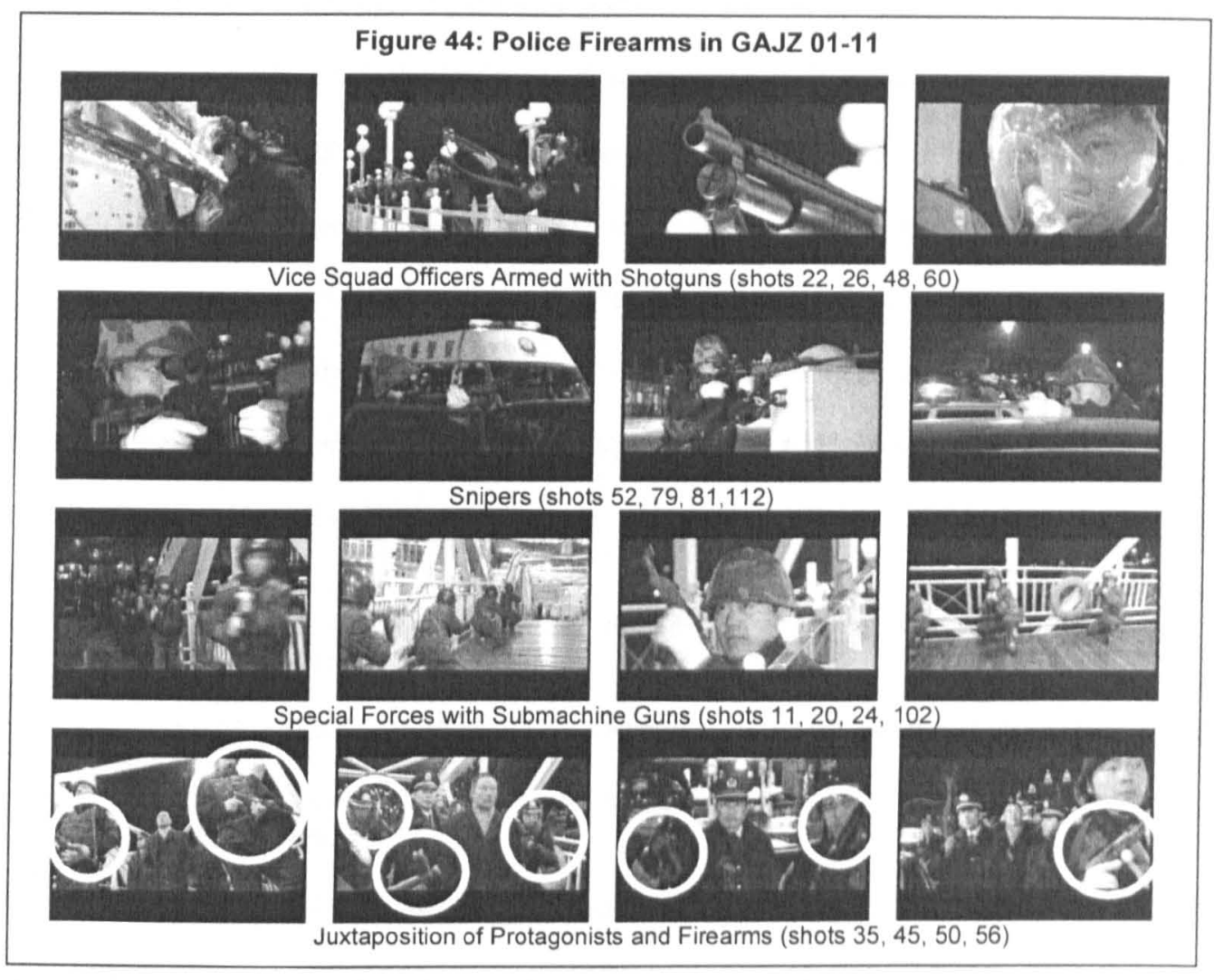

Before turning to the question why this may be the case, it is worth taking a closer look at another related narrative arc: Li Jian's first large-scale crime-fighting operation. This arc, which deals with the raids on various nightclubs throughout the city, spans across the final sequences of episode 4, as well as the first part of episode 5. I will be examining here Li Jian's announcement of the campaign (GAJZ 04-21), the staging of the operation (GAJZ 05-01), and its execution (GAJZ 05-03 and 05-06).

Sequence GAJZ 04-21 serves as the starting point for the operation and gives the character of $\mathrm{Li}$ Jian the opportunity to explain its relevance. The sequence is again underscored with the characteristic military march that was also used as an action theme in GAJZ 01-11. The cinematography of this one-scene sequence is similar to the one adopted for Li Jian's speech in GAJZ 08-05: there is little camera movement except for the first shot (which is used to 
establish the scene and is the longest shot of the sequence) and the last shot (during which the camera tilts slightly up- and downwards to keep one of the characters in the frame). The scene otherwise consists of static medium shots and medium close-ups at eye-level angel, showing either $\mathrm{Li}$ Jian as he gives his orders or the faces of those reacting to his orders. These shots are edited in a way that gives maximum emphasis to Li Jian's speech, for example by cutting to Huo Xiang (shot 05), Dai Wenqing (shot 06) and Fang Hua (shot 13) as Li Jian explains their individual responsibilities, or by paralleling a tri-colon sentence structure in $\mathrm{Li}$ Jian's speech with shots of his subordinates:

This operation shall be kept rigorously secret...

rigorously in line with the law... and shall seriously investigate any suspicious person. $\longrightarrow$ [medium close-up of Huo Xiang; shot 14]

[cut to Dai Wenqing; shot 15]

[cut to Lin Jun; shot 16]

Similar to sequence $01-11$, we again find repeated references to the military discourse, in this case verbally: First, the operation receives the code name "cloud sword" (shot 02), a reference on one hand to the fictional name of the city the drama is set in (Yundu 云都 can be translated into "cloud city") and on the other hand to Li Jian's first name (Jian 剑 meaning "sword"); second, Li Jian refers to the operation as "large-scale warfare" (dabingtuan zuozhan 大兵团作战; shot 03); and third, Li uses military terms such as "strike" (daji 打击; shot 01)， "battle front" (zhen 阵; shot 07), and "detachment" (zhidui 支队; shots 09 and 11) as he talks about the upcoming campaign.

This military theme is further developed in sequence GAJZ 05-01, when Li Jian launches the operation. Various visual and acoustic signs reinforce the militaristic nature of the operation (Figure 45): 


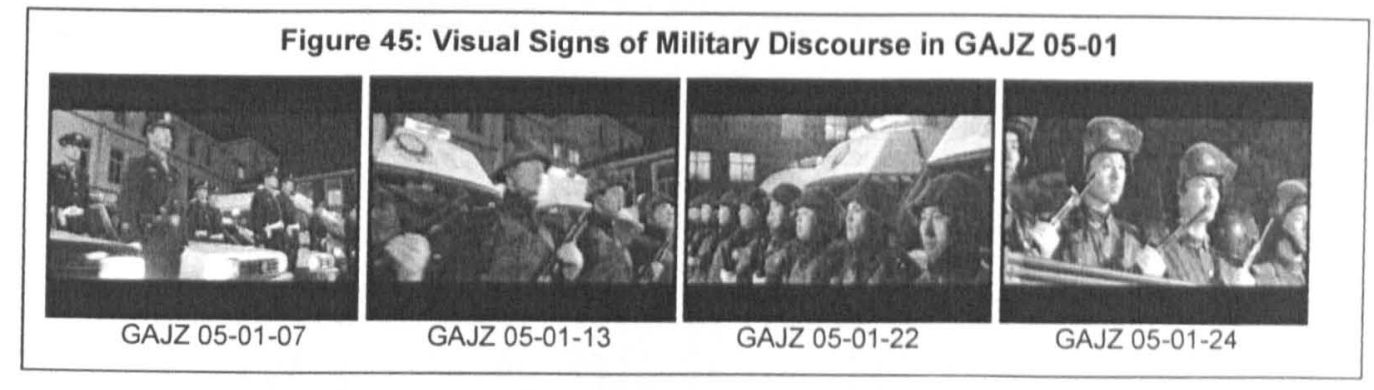

1) The police force is lined up like a battalion at a military parade, with the officers standing at attention next to or in front of their vehicles (shots $02-12$ ), the vice squad officers holding their rifles in a way that is common in military drills (shots $13-15$, as well as 21 and 22). The line-up concludes with green military transporters loaded with Special Forces troopers who are clad in green camouflage uniforms (shots 16 20, 24 and 26). In total, seventy-seven per cent of the shots in this sequence show some aspect of this police formation.

2) Low angles are used throughout the sequence to emphasise the grandeur of the police force (in fact, on average every third shot in the sequence adopts a low-angle perspective, see Figure 46).

3) Similar to the other sequences that feature military themes, the same characteristic military march is again used. The sequence's dynamics are constructed in a way that gives additional stress to this tune (for example by aligning cuts from one shot to the next with drum beats) while at the same time adding tension to the images of the police 'formation' (very high shot frequency throughout the shot series 21 - 24, 26 $27,33-34$ and $36-39)$. 


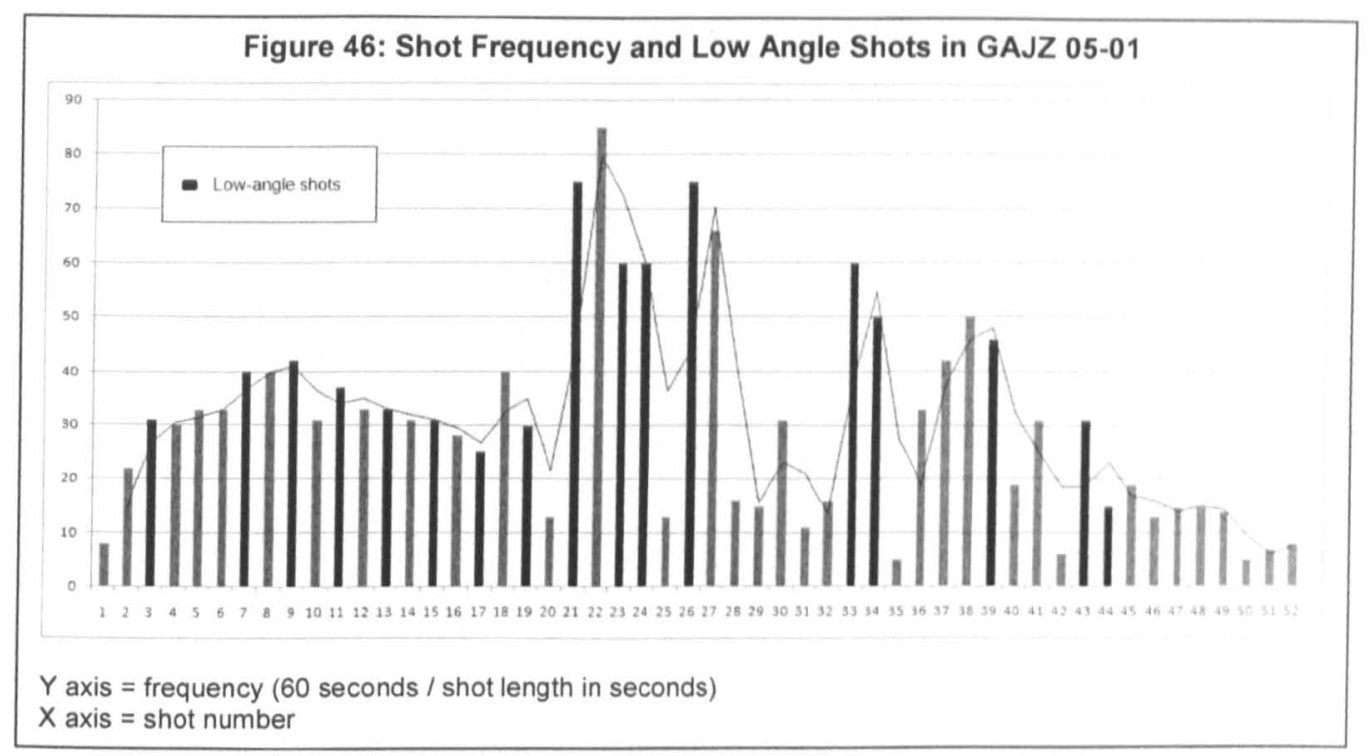

In the middle of the sequence, Li Jian addresses his troops, clarifying verbally the military nature of the operation by stating: "comrades, combat is imminent!" (tongzhimen, zhandou mashang yao daxiang! 同志们, 战斗马上就要打响了! shot 31). He then launches into a brief speech, which is accompanied by a surge in shot frequency (shots $35-42$ ):

Li Jian: Everyone knows that there are as many pairs of eyes here on earth as there are stars in the sky. The eyes of the masses are watching us attentively! We have to strengthen our quality within and act as models without! Strike at criminals, and stabilise society! I command every unit and every type of police to begin their operation in line with the scheduled program! Move out!

黎剑： 大家知道天上有多少颗星星, 地下就有多少双眼睛。人民群众的目 光在注视着我们! 我们要内强素质, 外塑形象, 打击罪犯, 稳定社 会。我命令各单位各警种按照预定的方案开始行动! 出发!

Note how the masses are again evoked here. Through the simile in the first sentence, i.e. that the operation is literally being watched by as many eyes as there are stars in the sky, the discursive statement is made that:

- The police force is under close scrutiny by the people. 
The articulation of this idea in this particular context is remarkable, considering that a few sequences earlier (GAJZ 04-21) the operation was deemed top-secret. The public scrutiny trope is then used here as a sign for the legitimacy of the upcoming law enforcement activities. Before ordering the troops to move out, $\mathrm{Li}$ Jian adopts a parallelism ( $4 \mathrm{X} 4$ characters) to state the general goals of operation "cloud sword": to improve the police force's own performance while simultaneously acting as a role model for others (within vs. without), and to strike at criminals while upholding social stability (attack vs. defence).

After the announcement and the launch of operation "cloud sword", the campaign is enforced in two sequences (GAJZ 05-03 and GAJZ 05-06). Both are devised as action sequences: fast-paced editing provides tension as police officers storm buildings (shots 07 14 in GAJZ 05-03, and shots 02 - 05 in GAJZ 05-06), as well as during a chase scene in a nightclub (GAJZ 05-03, shots 15 - 25). Shaky hand camera movements as well as low (in some cases Dutch) camera angles further add to the dynamics (GAJZ 05-03, shots 18 - 25). In addition, acoustic signs purvey suspense: the sounds of running men's footsteps, sirens, screeching brakes and car doors being slammed are featured throughout both sequences. The sequences are again underscored with the recognizable military march. Finally, an array of verbal signals with little actual relevance to the plot is used to clarify that the police are exerting authority. A loose juxtaposition of often contradictory commands is launched at the viewer as police officers arrest criminals, yelling: "don't move", "move", "freeze", "sit down", "take him away", etc. (the almost ritualistic repetition of such commands gives these otherwise mostly empty phrases the meaning: action is being taken; see GAJZ 05-03-05 and GAJZ 05-06-06). All of these signs are combined to make the discursive statement: the police are swiftly and efficiently fighting crime. Images of Li Jian overseeing the operation from a command centre filled with computers and surveillance screens (GAJZ 05-06, shots 01 and 10) further add the discourse fragment: 
- Yundu law enforcement is professional and technologically advanced.

However, the sequences are used to also make another statement; one concerning the nature of the public security threat in Yundu city (Figure 47). What was previously described merely as "illegal criminal activities" (weifa fanzui huodong 违法犯罪活动; GAJZ 04-21-01) committed by "suspicious persons" (xianyiren 嫌疑人; GAJZ 04-21-16) is now visualised. Three, possibly four offences are depicted visually in the two sequences, and only one of these offences is also expressed verbally. The first is prostitution, which is signified by a woman dancing for a group of costumers at a nightclub (GAJZ 05-03, shots 03 and 05) and later verbally reinforced by an officer announcing to a suspect: "I have the suspicion that you are involved in pornographic activities" (wo huaiyi nimen zher shexian seqing huodong 我怀疑你: 们这儿涉嫌色情活动; GAJZ 05-03-29). The second offence is gambling， which is signified by a group of men playing Mah-jong and counting money as the police storm in and arrest them (GAJZ 05-06-06). Third, the police officers arrest two men and find a plastic bag filled with white powder on their person, signifying drug trafficking (GAJZ 05-03-26). The fourth offence is only implied: at the club where the drugs are confiscated, three shots show the dance floor filled with ecstatically dancing young people (GAJZ 05-03, shots 07, 09 and 11), who are then detained by police officers (GAJZ 05-03-13). The erratic dancing and the sudden use of force against these individuals are possibly intended to signify illegal substance abuse. The drama then manages to not explicitly portray criminal activity, while still visually making the discursive statement:

- Public security is threatened by prostitution, gambling and drugs (or: huangdudu 黄赌). 


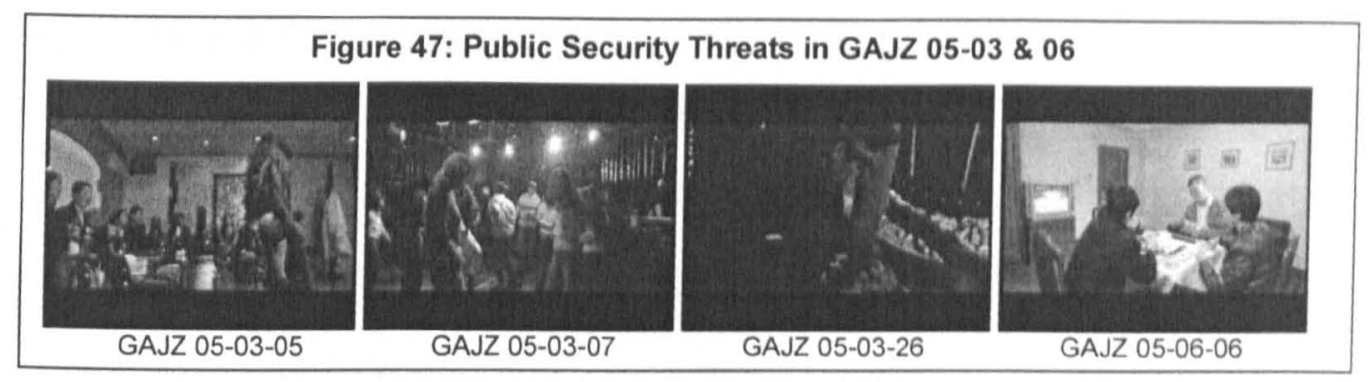

Similar to sequence GAJZ 01-11, the clash between the threat level that the police are faced with and the degree of force used on the side of the law enforcers is remarkable. This "largescale warfare" operation now sees hardly any struggle on the side of the criminals and no involvement at all of the camouflaged Special Forces troopers that were depicted so elaborately earlier. Instead, the offenders are coerced into cooperating through verbal statements or some minor use of physical force on the side of uniformed patrol officers. In fact, aside from a short chase scene in GAJZ 05-03, the entire operation is resolved with hardly any conflict.

The question then is: if the excessive amount of weaponry and military themes that are developed in most of these sequences is not relevant to the plot, then what are these signs supposed to signify? I would argue that the military discourse serves two functions: On one hand the dominance of martial themes is meant to propagate an image of the police force that those sponsoring the drama (particularly the Public Security Ministry) regard as desirable, i.e. one that shows the People's Police as a strong and well-prepared force which criminals should better not cross. On the other hand, it serves to instil awe in the audience and tap into their patriotic sentiment (particularly through the combination of glorifying military imagery with a pathos-laden musical score). In this sense, the commissioner Li Jian becomes more than a police official fighting crime, but functions as a military leader who goes to war to protect the stability of the nation. Similar to the drama HWDD, we are then confronted here with a juxtaposition of the security discourse strand with the discourse strand on national identity. 


\subsection{Visual Political Discourses in a Teen Drama}

The final part of my analysis focuses on the entirely commercial production of the teen drama NCNM (Tang, 2003). The twenty-episode series was written by the three screen writers Zhou Yong 周涌, Wang Qun 王群 and Zhang Wei 张勘，and was directed by the Hong Kong director Tang Jili 唐季礼. The drama was produced with no state involvement by purely private companies, headed by the firm Xingguang International (北京星光国际传媒). The series started broadcasting on CCTV-8 on 12 April 2003, and was aired each evening from 20:00 to 21:00; it ranked as the 11th most popular television drama of 2003 (Wang, 2004, 249). The drama is set in modern-day Shanghai and tells the story of the two young female university graduates Su La 苏拉 and Yan Ruyu 颜如玉 (also referred to as Yan Yan 颜颜)， who are trying to make a living in the metropolis. The plot primarily revolves around love, relationships as well as issues of welfare and consumption, and at first sight does not seem to strongly feature discourses on governance or security. However, a close analysis shows that even though these discourse strands are presented more subtly, they are nonetheless present. The discursive statements identified in this analysis, as well as the signs used to construct these statements, are summarised in the corresponding tables in Appendix 3.

\subsection{The Professional Bureaucratic State}

In contrast to the two series HWDD and GAJZ, the teen drama NCNM does not contain as many references to governance and official state or party leadership. However, it does include some information concerning administrative procedures as well as the concept of serving the people.

There are three instances in which characters from NCNM are confronted with the state. The first is the various characters' interaction with the law and with the Shanghai police throughout the drama; an issue which I will return to below in the context of security discourses (Section 4.4.ii). The second is a series of sequences that deals with various 
application procedures for Shanghai residence permits (NCNM 04-10, 06-21 and 06-22). The third occasion is portrayed in a narrative arc which tells the story of the heavily pregnant character Jing Sa 景䇋 applying for a pregnancy permit (NCNM 11-09, 11-10 and 11-19).

Let me turn first to my analysis of the residential permit applications shown in NCNM. Residential permits, or hukou 户口, play a role throughout the drama for various welfare reasons, which shall not be discussed here. Instead, I am interested in the portrayal of administrative procedures and government authority. An analysis of the respective sequences yields a result that at first sight deviates strongly from that of the other two programmes: the state hardly plays any role at all, and its relevance is merely implied by the general context. For instance in NCNM 04-10, the character Yan Ruyu seeks a lhukou for Shanghai after having secured a new job at a fitness club instructor in the city. For this purpose, she meets with the fitness studio's manager, who then takes care of the formalities for her. In the single-scene sequence, Yan Ruyu drops off the necessary documentation in the manager's office. The scene is generally very functional (shot 01 serves as an establishing shot, shots $02-04$ then show Yan Ruyu as she hands over the documents, and the rest of the sequence is assembled as a standard OTS dialogue with minimal correctional camera movement and no significant dynamic developments). However, a number of aspects about its arrangement are nonetheless noteworthy here. First, the mise-en-scène places the manager behind a large desk, with a bookshelf in the background; the man himself is dressed in a grey suit (shot 05). In this way the scene establishes visually that the manager is a professional businessman. Second, the two characters' posture is relaxed throughout the entire sequence; their exchange of the documents (visually marked by a close-up of the certificates being handed over in shot 03 ) and discussion of the procedure is accompanied by smiles and laughter, suggesting that the occasion is generally jovial. Third, the dialogue 
offers some insight to the extent of the formalities that the two characters are confronted with here:

Manager: Miss Yan, have you brought all your certifications?

经理： 颜小姐，你的证明材料都带来了没有？

Yan Yan: Yeah, I brought everything. Look, here's the diploma, a copy of the residential permit, the student ID, and the birth certificate. Look, is anything missing?

颜颜：恩，都带来了。您看，有毕业证、户口的复印件、学生证，还有身 份复印件。您看, 还差什么吗?

Manager: No, that's about it. If there are no complications, you'll be one of our formal employees by next week and you'll be a Shanghainese with a blue residential permit.

经理： 没有, 差不多了。如果没什么意外的话, 你卜周就是我们此式员 $\mathrm{I}$ 了。而且, 还是有蓝印户口的上海人。

Yan Yan: Haha, when that time comes I'll definitely invite you for a big dinner.

颜颜: 呵呵, 到时候啊, 我一定要请您吃一顿大饭。

Manager: $\quad O K$, it's agreed then. I'll be looking forward to it.

经理：好啊, 咱们就这么说定了, 啊。我可等者这一顿呢。

Yan Yan: Sure. I'll go back to work then.

颜颜：那当然, 我先出去工作了。

That the administrative procedure is simplified for dramatic purposes is in itself not surprising. However, the degree of the simplifications ${ }^{72}$, the general setting the conversation takes place in, the demeanour of the characters, the pleasant atmosphere that is portrayed in the sequence as well as the brevity of the scene (a mere 41 seconds) together make two discursive statements:

\footnotetext{
${ }^{72}$ For an insight as to how gross a simplification is being made here, see the guidelines for blue card hukouapplications on the Shanghai online information gateway (Goldaim, 2002)
} 
- Residence application procedures are regularly handled by Chinese employers in the private business sector.

- Under normal circumstances, residence applications are a standard procedure that requires little time, effort and formalities on the side of the applicant, and are not in any way unpleasant.

Whereas this first instance of a hukou application in the drama deals with a standard case, the second occasion involving such an application is more specific: it is a short narrative arc describing how the mother of the main character Su La is able to secure such a permit. In that section of the drama, Su La's mother, who is originally from Shanghai and had to move to Guizhou because of "historical issues" at a young age (a euphemism for having been forcefully relocated during the Cultural Revolution), wishes to move her residency back to her home city. This then becomes the theme of two sequences, in which the main male character Qiu Shi 邱石 is established as a resourceful and competent man who is worthy as a boyfriend of the female lead Su La.

The first sequence (NCNM 06-21) is used to raise the hukou issue and show how Qiu Shi decides to convince Su La's mother of his worth by solving her residency problems. The character is shown in the office of his friend, the police officer Zhao Lei 赵琵, as the two men talk about Qiu's relationship to Su La. After the obligatory establishing shot (shot 01) and some verbal bantering between the two characters to underlines their friendship (shots 02 03), Qiu moves into Zhao's office and sits down on his desk. The ensuing conversation then takes place in a very relaxed atmosphere (shot 04$)$. The dialogue is again visualised through medium close-up OTS shots, but it should be pointed out that the camera keeps a significant part of Qiu Shi's body in the frame as it shows Zhao Lei (shots 06, 08 and 10), and moves slightly closer to the character of Qiu Shi in the reverse shots (shots 05, 07 and 9). In addition, the shots of Qiu Shi are filmed from a low camera angle, which places the viewer in a position that almost coincides with that of Zhao Lei looking up at his friend from his desk (the perspective is not a point-of-view shot as such, but the angle nonetheless contributes to 
the general feeling of intimacy that characterises this sequence). The shot length ensures that Zhao Lei receives longer periods of dialogue to make his first two suggestions on how Qiu should impress Su La's mother (shots 04 and 06), while attributing slightly briefer periods to Qiu's nonchalant reactions (shots 05 and 07 ). As Zhao makes his third suggestion (i.e. that Qiu Shi should solve the residency problem for Su La's mother), the sequence slows down significantly (shots 08 and 09), showing how the suddenly pensive Qiu Shi asks about this option:

Zhao Lei: $\quad$ Man, I say, Shitou, how come at your age you still don't know what to do? If you really want to help Su La's mother thoroughly solve her problems, then nothing beats helping her get a residence permit.

赵否: 咬呀, 我说石头, 你怎么这么大年纪了还玩不能? 你如果真的想帮 苏拉妈妈彻底解决问题, 你不如帮她落个户。

Qiu Shi: Get a residence permit?

邱石: 落户?

Zhao Lei: Exactly.

赵䃌: 对呀。

Qiu Shi: You're the specialist in this area, help me think about this. Someone like Su La's mother, who moved away because of historical issues... can her residence permit be transferred back to Shanghai?

邱石: 你是这方面专家, 你帮我想想, 像苏拉妈妈这种, 因为历史问题而 去外地的人，能不能再把户口迁回上海?

The sequence then picks up speed again as Zhao Lei states that such an outcome was possible but would be accompanied with a lot of paperwork and as Qiu Shi decides that this is the right way to help Su La's mother (he storms out of the office, probably to fetch the necessary documents). However, even though Zhao's verbal statement and the increase in dynamics suggest that this hukou applications is more than a mere formality, this potential interpretation is immediately counteracted by three additional signs: First, by Qiu's slightly comical reaction (he suggests that Zhao Lei, being the expert, will have no problem solving 
the issue for him; shot 11); second, by Zhao yelling a friendly insult after his friend as he runs off (shot 14); and finally by the introduction of relaxed easy-listening music at exactly the point where the character's behaviour and dialogue would otherwise suggest an increase in tension (shot 12).

This narrative is then immediately concluded in the following sequence (NCNM 06-22), which depicts how Su La's mother pays a visit to her daughter and tells her that the residency problem is being resolved. The first few shots show Su La at work in her office as she receives the news of her mother's surprise visit (shots $01-03$ ). As her mother is being treated to some water by one of the employees (shot 04), Su La walks through the travel agency she works at to welcome her visitor (shot 05; this is arranged as a long shot with substantial movement and serves the function of establishing the general setting and miseen-scène). The rest of the sequence is again a relatively conventional dialogue scene that focuses strongly on the mother's verbal statements that Qiu Shi was able to take care of the administrative procedures quickly and effectively, or as she puts it "with just one word" ( $y$ i ju hua 一句话). The dialogue then continues as follows:

Su La: But is he not a business man? How come he can help you solve the problem by just saying one word?

苏拉: 他不过就是个生意人嘛? 怎么样一句话帮你解决的?

Mother: Oh, he's really very skilled. Haha, oh, he said to others: 'this lady was born and bred in Shanghai. The hospital still has her birth certificate.' That way he was able to fetch my certificates in one go. Tell me, is he not really skilled?

母亲：嗼呀, 他能耐可大着呢。哈哈, 呅呀, 他呀跟人家说: 这位女1:尘 上海土生土长的上海人。医院里还有出生证明呢。这他们什么办 法, 把我的证明啊一下子就拿来了。你说他本束大不大呀?

What is noteworthy about all three of the above sequences is on one hand that the state is hardly personified at all (merely indirectly through the character of police officer Zhao Lei, but even he is shown in a relaxed and even slightly comical context, and does not fulfil any 
official functions), as well as on the other hand that the 'common people' (represented here largely by entrepreneurs) are able to handle administrative procedures without much effort or complication (Figure 48).

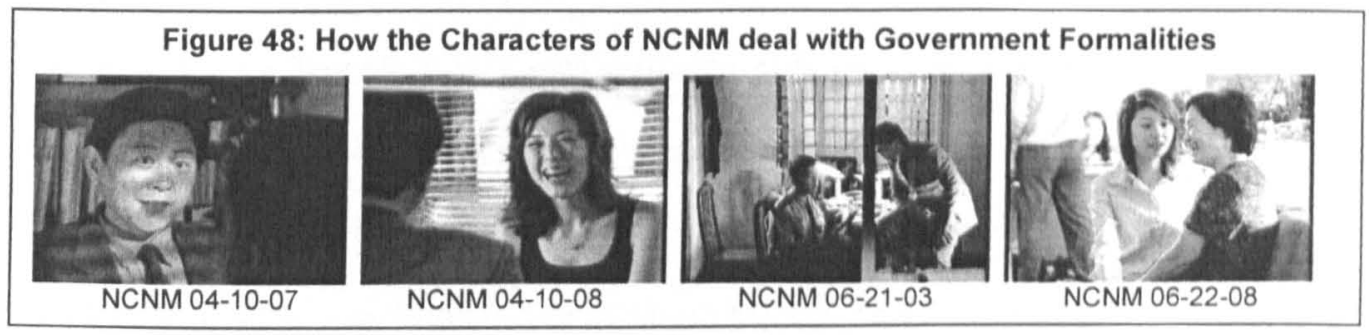

Another narrative arc in NCNM deals with the application procedures that one of the characters is confronted with as she applies for a pregnancy permit. In the three sequences of this arc, the discourse on state administration is presented more explicitly, and is linked with the drama's gender discourse. However, in this case the issue is not resolved as easily.

The first of the sequences (NCNM 11-09) is used to address the general crisis that one of the characters faces: the pregnant Jing Sa has been left by her husband, and is now encountering problems as she tries to register with a hospital for the delivery of her baby. The person of authority in this sequence is not a government official but a female doctor. The sequence starts with two establishing shots of the hospital, and then continues with a three-way dialogue scene between the doctor, Jing Sa and Jing Sa's friend Su La, which is set in the doctor's office. Some camera movement is used in the medium shots of the scene to show all three characters seated around the doctor's desk (shots 04, 05, 08, 10, 15 as well as the final shot 16), but otherwise the medium close-ups of the individual faces are kept static. The doctor's attire (lab coat, stethoscope around the neck and a pen in hand) as well as the arrangement of the office setting (a desk featuring two phones, some medical equipment, a line of books and an open file-case in front of the doctor) suggests that the girls are confronted with a professional. At the same time, the woman's demeanour and speech, as 
well as the use of high angles in various shots (shots 04,08 and 15), indicate that she is impatient, patronizing and generally looking down on the two girls. The dialogue then clarifies the extent of the problem, and the doctor's view of the situation: Jing Sa cannot have her baby delivered without a pregnancy permit, and that has to be obtained at the local residence office. When the doctor realises that the girls are confused by these formalities, she laughs and says: "There's no way for me to explain this to you. How about this, you go and get your husband and I'll explain it to him, maybe he will understand." (wo gen ni ye shuo bu qingchu. Zheyang ba, ni qu ba ni laogong lai, wo gen ta jiang, yexu ta neng dong. 我跟你也说不消 楚。这样吧, 你去把你老公交来, 我跟他讲, 也许他能獚。) Jing Sa informs the doctor that she is not married, which then leads the medic to deride the two girls for being irresponsible (shots 12 - 14). The general humiliation of this sequence is reinforced through quick medium close-ups of Su La's embarrassed face (shots 03, 07 and 12) and Jing Sa's offended reaction (shot 14). The sequence concludes with the two upset girls leaving the hospital and heading for the government office of their residential district.

The second sequence (NCNM 11-10) shows the girls trying to apply for the required pregnancy permit at the local government office. An establishing shot shows the doorway to the social service centre at Shimen Road Number 2, and a second shot focuses on the door sign to the office. The rest of the sequence then largely consists of medium close-ups of the girls' embarrassed faces and of the annoyed female official who is dealing with their case. Similar to the previous sequence, this scene again shows the person of authority shocked by Jing Sa's marriage status and then has the official scolding the two girls for being irresponsible. It is made clear to Jing Sa that she needs her former husband's credentials in order to receive a pregnancy permit. Since the husband has already left the country, the two girls are again unable to overcome this crisis. 
What is significant here is how this conflict is finally resolved. Jing Sa's condition compels the character Zhao Lei to demonstrate his noble personality by offering to marry her (NCNM 11-15). When they return to the government office together, the situation changes significantly (NCNM 11-19): after Zhao Lei entices the female official with his patients and friendly attitude (as well as with a reference to his profession as a police officer), the formalities are concluded in less than thirty seconds (shots 6-13). The procedure is essentially simplified to close-up shots of certificates being exchanged (shots 6 and 12), a close-up of a red stamp being applied to one of the documents (shot 10) and medium close-ups showing the applicants' faces (shots 09 and 13). In the end, two signs are juxtaposed to ensure that the viewer interprets the scene positively: on the one hand the visual sign of Zhao Lei and Jing Sa's laughing, happy faces and on the other hand the acoustic sign of a romantic melody setting in after the permit has finally been stamped (Figure 49).

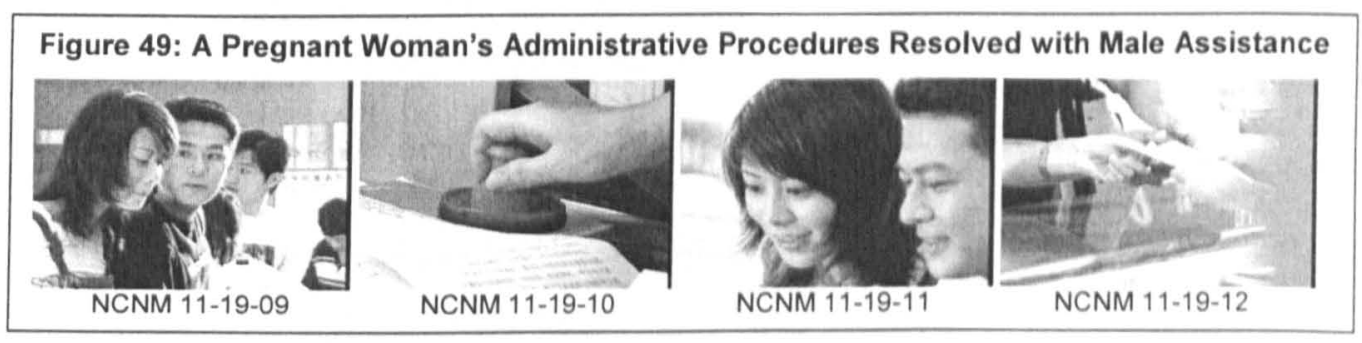

In this sense, Jing Sa's experiences in these sequences are not used to criticise the Chinese state or its degree of bureaucracy. Instead they help characterise one of the main figures by offering the second male lead Zhao Lei a chance to demonstrate his value as a potential provider. The ease with which the red tape is finally resolved again suggests that administrative issues are easily settled in modern-day China, at least if one is a man.

What is then most striking about both the hukou application theme and the pregnancy permit narrative is their gender dimension: None of the actual formalities are handled by the female protagonists. Instead, the procedures provide the backdrop for men to demonstrate their social value by solving the conflicts: The pro-active character of Qiu Shi obtains the residence 
permit for Su La's mother, the honourable police officer Zhao Lei obtains the pregnancy permit for Jing Sa, and even the otherwise assertive female character of Yan Ruyu has her male employer handle her hukou application. When confronted with the state, women are shown as incapable of dealing with the administrative procedures, and the officials involved are portrayed as being impatient with the suggested degree of female naivety.

Even though this thesis does not focus on gender discourses, the case of NCNM is nonetheless a good example of how competing discursive positions vie for hegemony within a popular television drama: In many sequences, the drama takes a very strong feminist position on gender issues, but in the end conservative themes dominate..$^{73}$ The reason for this is that the screenwriters' personal preferences compelled them to include some emancipated themes, but that the drama's marketing required conservative conclusions in order to ensure the series' appeal to the generally older target audience of TV dramas in China (19, 62:01 68:29). This shows what a powerful role the production companies' perceptions of CCTV drama audience demographics play during the formulation of discursive positions.

\subsection{Security Issues as a Comical Plot Complication}

In comparison to the dominant role that public security issues play in GAJZ and foreign security issues have in HWDD, the most striking aspect of the security discourse in NCNM is its apparent absence. Foreign security issues are not dealt with at all in this teen drama, and public security is merely a minor side-issue. In contrast to GAJZ, no explicit verbal declarations are made concerning, for example, the police's service to the people. However, the discourse fragments that deal with public security in this drama are not entirely different

\footnotetext{
${ }^{73}$ The way in which the girls are treated by the doctor in NCNM 11-09 suggest a critical attitude towerds arrogant and sexist persons in power, but the fact that the girls' problems are resolved not by them but by men undermines this originally feminist sentiment. As an even stronger example, consider the sequences in which the main female character Su La discusses marriage roles and gender issues with various other characters towards the end of the series (NCNM 18-03,18-04,18-12, 18-13, and 18-14), and then decides not to marry Qiu Shi because she fears it would curtail her independence (NCNM 18-23 and 18-24). However, the confilcts are all resolved, and the majority of characters marry in the end (Jing Sa marries Zhao Lei in NCNM 15-23; Su La marries Qiu Shi in NCNM 20-22; Xlaofang accepts Lo Ke's marriage proposal in NCNM 20-12). The only character who is not married at the end of the series is the tragic figure Yan Ruyu (her divorce highlights her personal failings and functions as a punishment for her antagonistic behaviour throughout the drama).
} 
from those used in the main melody propaganda drama GAJZ, even if they are more implicit. To analyse these discursive statements, it is worth examining two types of sequences: the ones that feature law enforcement activities, and the ones that deal with the character Zhao Lei and his work as a police officer.

Let me turn first to the character of Zhao Lei. The few short sequences that show Zhao Lei working as a police officer depict him as a friendly professional who is dedicated to serving the public (NCNM 01-08, 01-13, 03-18 and 06-17). This mainly serves to underline this character's general personality traits: throughout the drama, Zhao is established as a compassionate and responsible person (for example on the various occasions that show him caring for Jing Sa; NCNM 05-08, 05-09, 09-02 and 11-19). His main function is arguably as the main male character's best friend and a source of advice in moments of conflict. For the majority of Qiu Shi's relationship problems, the male lead turns to Zhao Lei, who then helps to put the problem into perspective. Note how these sequences are often set in Zhao Lei's workplace, i.e. either at his office at the police station (NCNM 01-14, 03-10) or on the street as he is patrolling a neighbourhood in Shanghai (NCNM 06-17). In these cases, Zhao Lei is shown wearing his police uniform and doing his duty as a civil servant as he hands out words of wisdom. The most extreme case of this character's function as a moral compass for those around him is shown towards the end of the drama series: after having been injured during a law enforcement operation in Yunnan, Zhao spends two episodes in a coma. 'The actual circumstances of Zhao Lei's injury are not shown in the drama, but Zhao's commitment and heroism are implied both through telephone conversations during his absence (NCNM 16-04, 16-19) and the melodramatic scenes that show his return to a hospital in Shanghai (NCNM 17-03, 17-04). When Zhao Lei awakens towards the end of episode 19, he realises that the marriage between his friends Qiu Shi and Su La was cancelled because of 
Qiu Shi's stubbornness (NCNM 20-01). Once again it is Zhao Lei who talks sense into the main male protagonist (NCNM 20-02, 20-14) and saves his friend's relationship.

Through actions such as these, Zhao Lei's character is constructed as virtually flawless, especially next to the main male protagonist Qiu Shi: Zhao is shown as caring, amiable, responsible, self-sacrificing and wise. In this regard, Zhao's profession as a police officer is used as an additional device to reinforce these character traits, thereby making the discursive statement: an honourable and friendly man such as Zhao Lei would be likely to also be a police officer (Figure 50; note how the idea of 'serving the people' is not verbally expressed in any of these sequences, but how images of Zhao Lei riding his bicycle through the neighbourhood community or helping out an old lady nonetheless imply such a meaning).

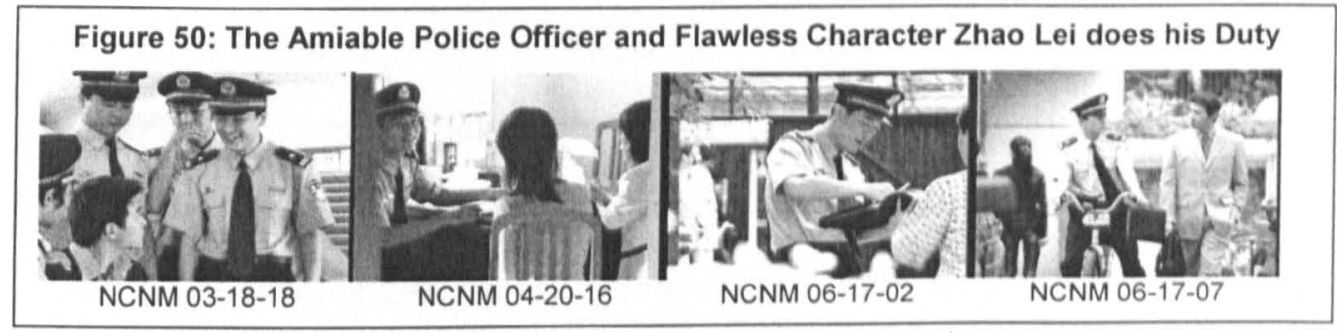

Next, it is worth examining more closely the occasions on which law enforcement activities play an explicit role in the series. In addition to being friends with law enforcement official Zhao Lei, the characters of NCNM are confronted with security issues in two other ways: either directly as a plot complication or indirectly when police presence is a background theme.

The first case can be found in two narrative arcs, as well as one brief sequence: the brief sequence deals the two girls Su La and Yan Ruyu trying to find accommodation by placing hand-made posters on walls throughout Shanghai's former French Concession quarters, and then being chased away by the local neighbourhood watchmen (NCNM 01-16). The 
watchmen are only shown very briefly, and the whole sequence is given a non-threatening air through its dynamic and upbeat musical score.

The first full narrative arc that deals with public security involves the characters Su La and Yan Ruyu mistakenly being arrested for human trafficking. The second narrative deals with Qiu Shi repeatedly returning to a metro station in Shanghai where he first saw his dream girl $\mathrm{Su} \mathrm{La}$. When he finally sees her again, he is detained by the police for loitering. Both conflicts are again resolved by police officer Zhao Lei. To exemplify what discursive statements on security issues these narrative arcs make, I will analyse the key sequences of each arc (NCNM 01-13 and 01-15 for the first narrative, NCNM 03-18 for the second).

The sequence NCNM 01-13 is set at the Shanghai Railway Station and shows how the girls take shelter in a public place after having lost their dormitory apartment to a fire. The sequence consists of three different scenes, which each possess their own dynamic arc: The first introduces the setting with a series of brief establishing shots (shots $01-05$ ) and then shows Su La making a phone call to her mother from a pay phone at the station (shots 01 16). The discursive themes of this first part are family, employment and perseverance in the face of adversity. The tone is generally melancholic (signified by slow fades between shots, slow camera movements, and a poignant flute melody playing throughout); security issues play no role at this point. The second scene has Su La walking back to her friend Yan Ruyua, who is sitting in the waiting hall of the train station. As the melancholic flute music fades out and gives way to the background noise of the train station, the two then talk about Su La's ex-boyfriend Ying Shan, and about her relationship to her mother (shots 17 - 28). While this section again focuses on discourse strands such as family and relationship, it also introduces the security theme that will later move to the foreground: in shots $23-26$, a group of men and women enter the station's waiting room, carrying babies on their arms. Note how these four shots are underscored by a suspenseful tune. This acoustic sign serves to mark the 
group as a threat; an association that otherwise would not be supported by the visuals alone (the group theoretically pass as a migrant family from the country-side). As the men and women sit down near Su La and Yan Ruyu, the two girls continue their conversation, dreaming of a big, comfortable bed.

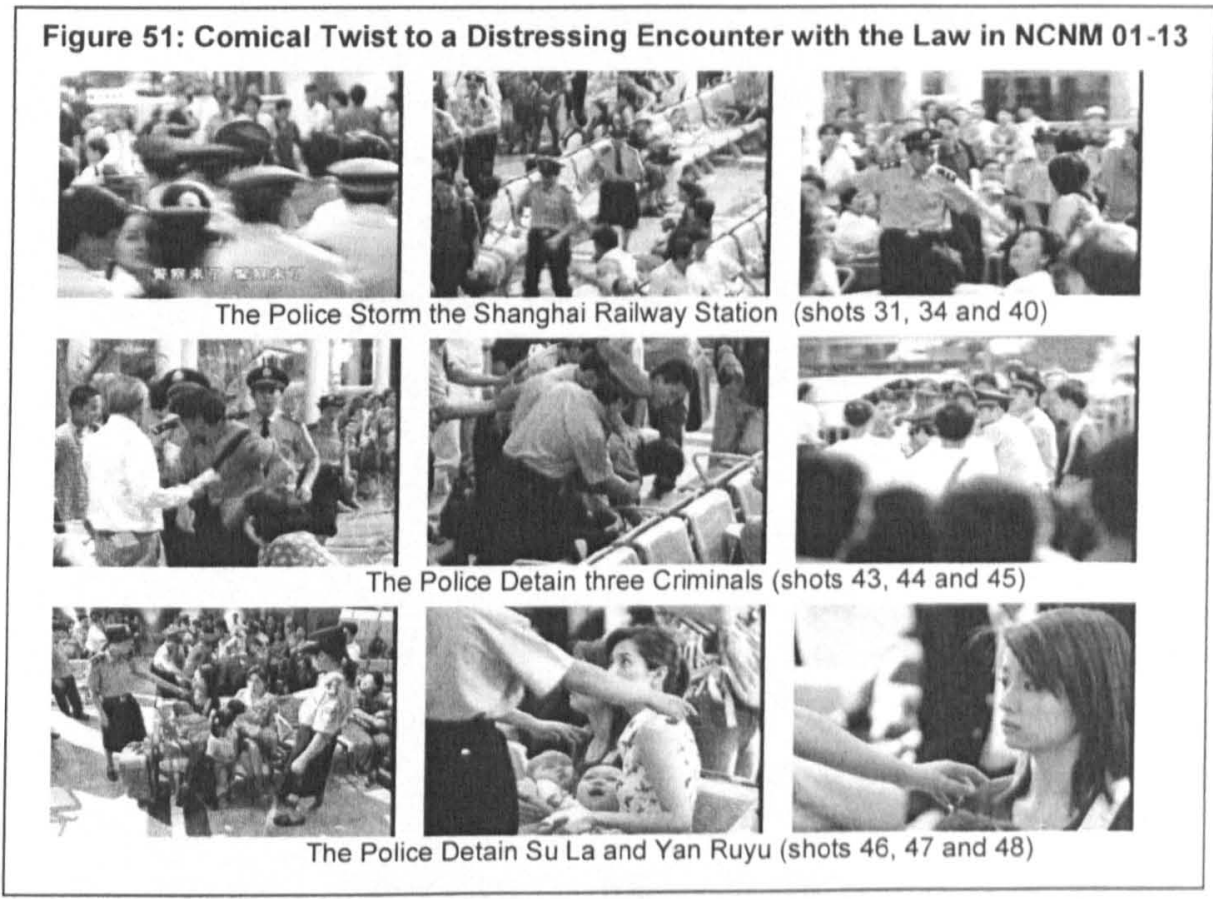

At this point, the third scene commences. Police officers storm into the waiting area, and the shady group of men and women start to run, after handing the babies to the baffled Su La and Yan Ruyu (shots 36 and 38). Increased shot dynamic, action music, rapid camera movements and the sound of the babies crying underscore the urgency of the scene. To relay the fact that the viewer is witnessing a police raid, two verbal signs are relentlessly repeated (shots 33 - 37): police officers yelling "freeze" (zhanzhu 站住) and "don't run" (buxu pao 不许 跑; with the minor alteration bie pao 别跑). The last part of this scene uses no verbal signs at all (shots $39-48$ ). Instead, it shows how the fleeing men are apprehended by the police, and how female officers surround Su La and Yan Ruyu. As the men are struggled to the ground by male officers (shots 43,44 and 45 ), the two female officers merely tap the girls on the 
shoulder, a gesture that here signifies 'arrested' or 'detained' (shots $42,46,47$ and 48). In this sense, two opposed themes clash: on the one hand, the juxtaposition of shot dynamics, acoustic signs and camera movements gives the scene a threatening and potentially dangerous air; on the other hand, the girls' situation receives an almost comical connotation through the confused expression on their faces as they hold the babies, and the non-violent, mild manner in which they are arrested by the two female police officers (Figure 51).

A similarly light connotation can be found in the conclusion of this short narrative arc, the sequence NCNM 01-15. This sequence is constructed as five different scenes, all set in or outside the police station that the character Zhao Lei works at:

$\begin{array}{lll}\text { Scene 1: } & \text { Shots 1-16 } & \begin{array}{l}\text { Su La and Yan Ruyu are } \\ \text { interrogated by two officers. }\end{array} \\ \text { Scene 2: } & \text { Shots 17-28 } & \begin{array}{l}\text { Qiu Shi's car breaks down as he } \\ \text { and Zhao Lei are about to drive to } \\ \text { dinner. }\end{array} \\ \text { Scene 3: } & \text { Shots 29-33 } & \begin{array}{l}\text { Zhao Lei finds out about the girls' } \\ \text { arrest and the two are set free. }\end{array} \\ \text { Scene 4: } & \text { Shots 34-48 } & \begin{array}{l}\text { Qiu Shi sees the girls get into a } \\ \text { taxi, but is unable to stop his } \\ \text { dream girl before she drives off. }\end{array} \\ \text { Scene 5: } & \text { Shots 49-66 } & \begin{array}{l}\text { Qiu Shi asks Zhao Lei for help in } \\ \text { finding Su La. }\end{array}\end{array}$

Scenes 1 and 3 each explicitly deal with security issues. The first shot of scene 1 is a lengthy establishing shot during which the camera moves through the police station waiting room. The shot derives its significance from the mise-en-scene, taking in first the two criminals from NCNM 01-13, who are shown locked up in a cell, then female police officers caring for babies, and finally the entrance to the interrogation room. In this fashion, the camera moves from the crime (and its punishment) to the victims and how they are being cared for, and finally concludes with the protagonists and their role in the situation. The following shots are arranged in a way that emphasises the confrontational nature of the scene (Figure 52): 
medium shots showing the two girls (shots 03 and 05) are pinned against a mirrored perspective of the two interrogating police officers (shots 02 and 04); a main shot establishes the line of sight between the two groups as they look at each other across a table (shot 10; note how a window frame divides the image into two distinct sections: the left one showing the officers, the right one showing the girls); and finally a number of medium close-ups of the girl's embarrassed faces (shots $11-13,15$ and 16) is juxtaposed with low angle medium shots of the interrogators (shots 08 and 14). The entire section of shots is dominated by the colour blue and is characterised by relatively hard lighting as well as stark light-dark contrasts. For instance, the scene makes distinct use of shadows (note the shadows on the wall behind the two officers in shots $02,04,08$ and 14) and features backlighting created by the office lamp on the interrogators' desk (shots 08 and 14). Acoustically, the scene features the sound of babies crying in the background.

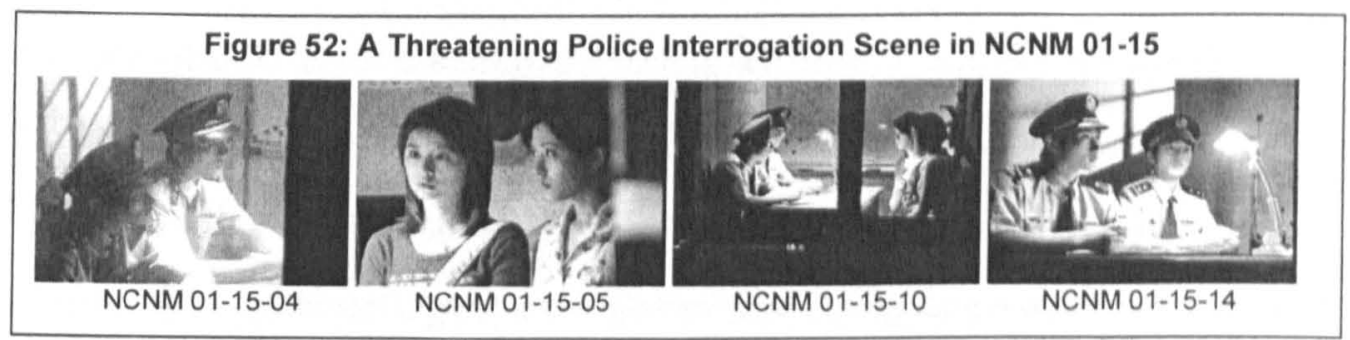

The visual and acoustic signs first seem to imply a threatening situation. This impression is further enhanced by the demeanour and verbal statements of the police officers. Every excuse the girls present is questioned by the officers, and when Yan Ruyu threatens to sue the police for having unlawfully detaining her, she is bluntly told that she had better not threaten legal action in a police station (shot 08 ; it is thereby suggested that due to the gravity of the crime, the girls are in no position to demand anything). All of these signs are used here to emphasise the severity of the situation. However, this severity is then quickly dissolved, first through the general absurdity of the misunderstanding (the two university students being mistaken for traffickers of infants, and the police officers not believing their 
story), and second through an acoustic sign: when the girls' explanation culminates in them having to admit that they do not possess any means of identifying themselves (their credentials were destroyed when their dormitory burned down), a comical melody sets in and gives the entire scene a light air.

From that point on, the sequence moves away from the graveness suggested in the first shots, and becomes much less serious. The next scene shows how Qiu Shi's car breaks down outside the police station, just as Qiu and his friend Zhao Lei are about to drive to dinner in Qiu's car; again, the scene features the comical melody, as well as the light bantering between the hungry Zhao Lei and his friend Qiu Shi. Zhao is then called back into the station to confirm the two girls' identity (he met them when they reported the fire at their dormitory). This third scene further increases the comical theme, first through the continuation of the comical melody that plays in the background, second by having Zhao pat the little babies as he walks through the waiting hall (shot 29), and then through shots 30 to 33, when Zhao sees the girls and they all laugh as the situation is resolved. Note how additional acoustic and visual signs are used to enhance the impression that all is now well (Figure 53): no additional high or low angles are used; the lighting is softer and dominated by a warm, orange colour; the two interrogators are no longer shown, and neither are the table or the blinding desk lamp that dominated the first scene. Furthermore, the background melody pauses briefly as Zhao Lei enters the room (shot 30), and then starts again as the two girls recognise Zhao and jump up in joy (shot 31). The scene ends with the girls looking embarrassed as Zhao Lei laughs at them (shots 32 and 33). Any further official formalities are now only implied, and not explicitly shown. The next shot has the two girls leaving the police station, and the entire conflict has been resolved in 18.8 seconds. 


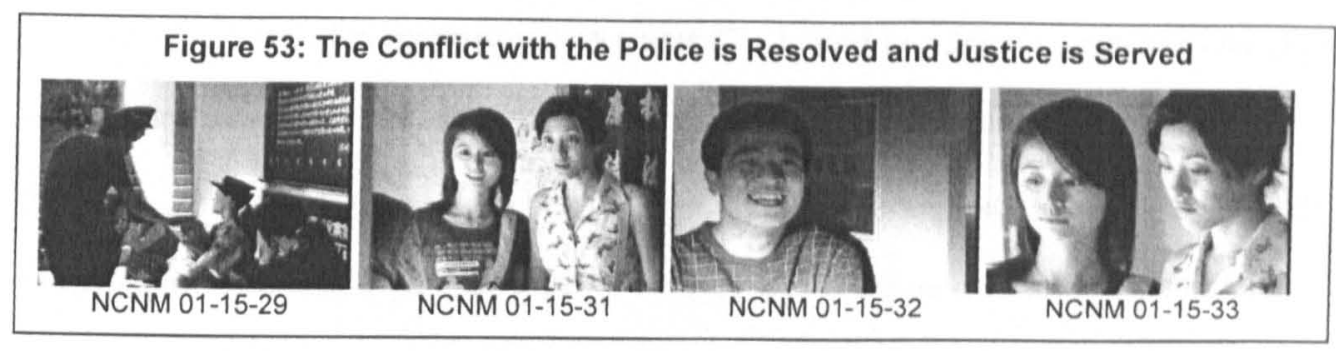

The rest of the sequence is then used to further develop the love story between Su La and

Qiu Shi, and only makes two verbal statements on law enforcement: Yan Ruyu voicing her anger at having been unjustly detained, and Su La calming her down by stating that everything had been resolved and that the police had apologised for the misunderstanding (shot 34). Both these statements do not imply here a particular discursive position towards police work, but instead serve to highlight Yan Ruyu's impatient nature and Su La's forgiving and mild character. They also tie straight into the discussion on relationships and the gender discourse fragments that now follow, with Yan Ruyu stating that she feels compelled to either find herself a "a knight in shining armour who is handsome, rich and has a car" (you shuai you you qian you you che de bai ma wangzi 又帅又有钱又有车的白马王子), or to return home to the countryside and get married. The statement here is that women can only find stability and safety either in a relationship that facilitates a lifestyle of consumerism or in the protective setting of rural marriage.

What the viewer is then left with after this sequence is the impression that confrontations with the law merely help drive the overall plot forward. This can be paraphrased as the following discursive statement:

- Confrontations with the law are minor comical distractions in life. The same usage of the law enforcement discourse can be found in NCNM 03-18, although in this sequence additional emphasis is placed on the professionalism of the police. 
The sequence is divided into two scenes (shots $01-10$ and 11 - 24): First, Officer Zhao Lei receives a phone call at his office, informing him about a conspicuous man loitering at a Shanghai metro station. Zhao and his colleagues then grab their hats and rush out of their office. Second, Zhao and his men observe the suspect via the metro station's surveillance cameras. When they apprehend the man, Zhao realises that the suspect is his friend Qiu Shi, and that Qiu had merely been searching Shanghai's subway stations for the woman of his dreams, Su La. But before the sequence takes the usual upbeat comical turn towards the end, an array of visual and acoustic signs are used to construct a suspenseful crime-fighting story.

The first of the two scenes contributes to this overall theme by strongly featuring increased camera movements (shots 01, 02, 03, 07 and 09) and fast shot dynamics (the fastest shot of the sequence is the one in which Zhao Lei grabs his police hat; shot 08 ). In this way, the pace of the action is increased. Also, the confined space and crowded mise-en-scène add the sense that the police station is an industrious place full of hard-working law enforcers (Figure 54); note how the camera repeatedly cuts across all three of the officers and their work spaces, and often keeps additional personnel in the background of the frame as Zhao Lei is going about his business, for instance in shots 04, 05, 07, 09 and 10 .

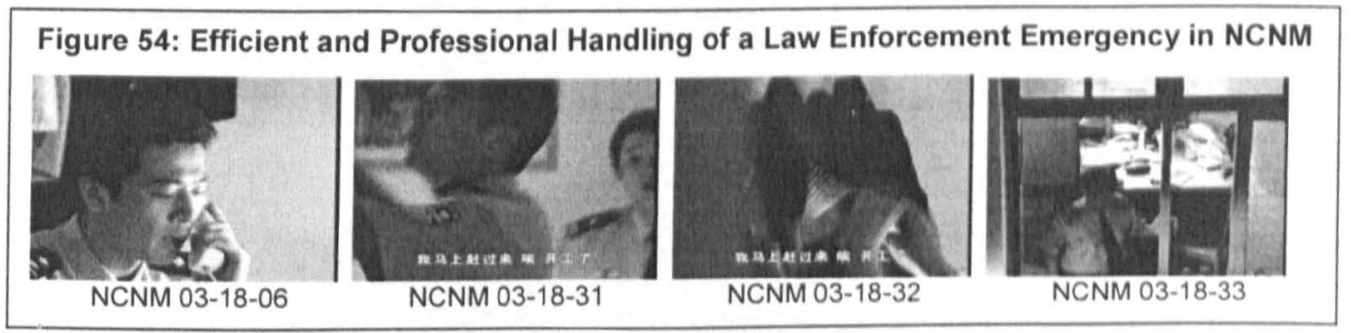

Throughout the scene the only character who has spoken text is Zhao Lei. The initial part of his de-facto monologue is the (implied) telephone conversation with an employee at a Shanghai metro station. Note how the text consists of short, elliptical clauses that are spread across a total of four shots (shots $03-06$ ): 
Zhao Lei: "Hello? Police station. A suspect has been sighted at the metro station. Ok. Address? Ok. You stall him first. I'll be over immediately."

赵磊：“喂? 派出所。地铁里发现一个嫌疑犯。好。地址? 好, 你先稳住 他啊, 我马上赶过来。”

After Zhao hangs up the phone, the dynamics pick up and he announces to his colleagues:

"Hey! We have work!" (ai! kaigongle! 唉! 开工了! shot 07), and then repeats the earlier

statement: "A suspect has been sighted at the metro station" (ditieli faxian yi ge xianyifan 地铁

里发现一个嫌疑犯; shot 9). The suspenseful music that now sets in further underscores that

the time for action has come. The general discursive statement here is that:

- The police are assiduous professionals who are ready at a moment's notice.

The next scene continues the statement on the professionalism of law enforcement officials, before then resolving the crisis in a similarly light fashion as seen in NCNM 01-15 (Figure 55). Again underscored by the same suspenseful musical theme, the scene builds up an air of foreboding through slow and largely equally paced shots of Zhao Lei and his men as they assess the situation at the metro station and apprehend the suspect (shots $11-16$ ). What particularly adds to the sense of a professional law enforcement intervention here is the use of images-within-images, i.e. of shots that show the action through surveillance screens (shots 12, 14 and 16). In this fashion, the viewer sees what Zhao Lei is seeing (POV shot): a suspect standing on the metro platform and the police rushing in to apprehend the man. This signifies a general 'watchfulness' of law enforcement officials. In addition, the use of surveillance camera shots links the public security discourse to the discourse on technology, suggesting that law enforcement in Shanghai is technologically advanced. This is further amplified by the relatively cold, blue colour-scheme throughout these shots, and the miseen-scène: the background of the surveillance room in which the action is set is dominated by flashing dashboards. 
Once Zhao Lei walks down to the platform, the suspenseful music fades out (shot 18) and the scene is resolved with smiles and laughter (shot 21). The colour-scheme changes from the former cold blue filter to a much warmer red colour. In addition, the camera angles (medium close-up shots that are angled in a way that almost offers POV perspectives of Zhao Lei, and the scene concludes with two OTS perspectives) are used to create an intimate feeling between the characters of Qiu Shi (the potential suspect) and his friend Officer Zhao. Humour is provided through the verbal statement by Qiu that loitering at the metro station was, in a sense, Zhao Lei's idea, as well as by the other officers patting Qiu on the back when they realise that they accidently arrested a famous millionaire.

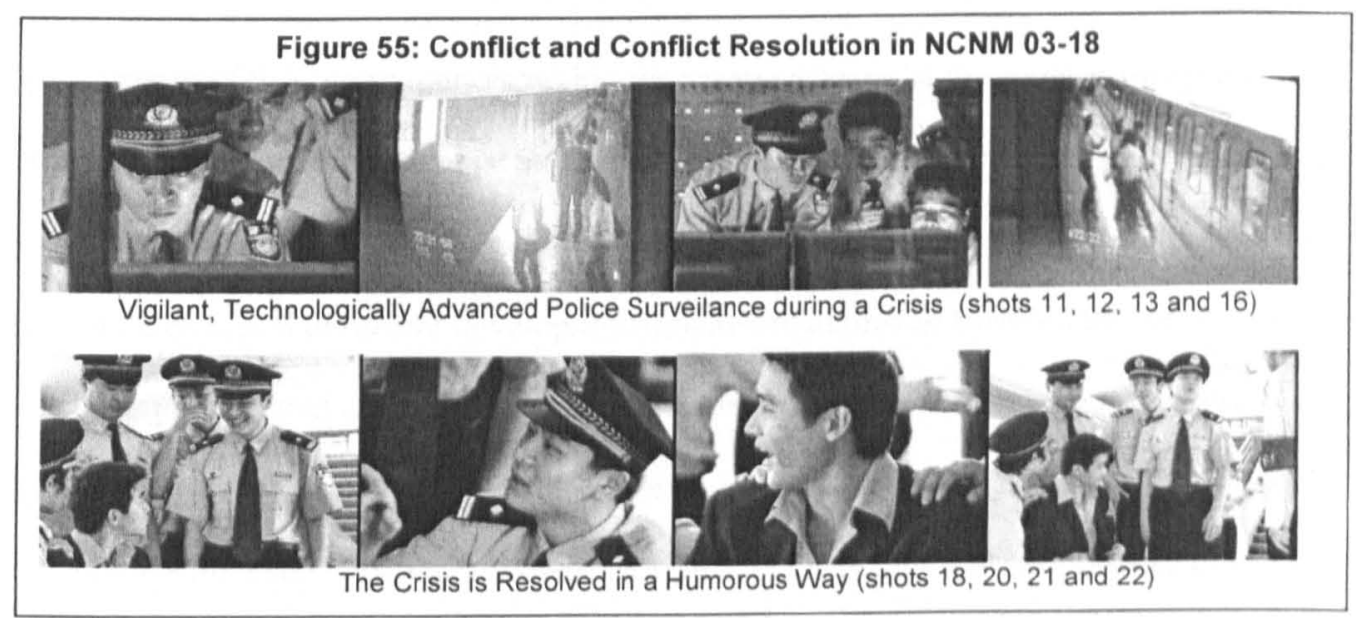

The comical touch to this sequence again shows that public security issues only functions as a mild distraction to the general plot development in NCNM (an additional example can be found in NCNM 02-05). Confrontations with the law occur when characters are in the wrong place at the wrong time, and although such conflicts can cause considerable inconvenience, the general impression is that the police are merely doing their job, that they are vigilant, trained professionals with technologically advanced equipment, and that justice prevails in the end.

A final case of the public security discourse in NCNM that needs to be addressed is the background presence of law enforcement during various sequences. In none of these cases 
do the police provide plot complications, but their presence nonetheless contributes to the security discourse. The most prominent example of this can be found in episode 7 , during a narrative arc dealing with an accident in which a travel agency bus from Su La's company was involved (for another example see sequence NCNM 01-07, during which the police help handle the fire at Su La's and Yan Ruyu's university dormitory). In the sequences NCNM 07. 14 and 07-17, Su La and her boyfriend Qiu Shi travel to the crash site to assess the situation and handle the travel agency's responsibility for the accident. Upon Su La's and Qiu Shi's arrival, the police are busy securing the crash site, while various helpers and medics are tending to the victims.

The purpose of the two sequences within the greater scheme of the series is threefold: to show how the female protagonist Su La deals with a professional crisis, to give the male lead Qiu Shi an opportunity to take charge of a complex situation and prove his courage in the face of danger, and to further develop the romantic relationship between the two characters. Also, the professional crisis that is introduced here is developed throughout the next two episodes of the series and provides the stage for circumstances that later cost Su La her job (NCNM 09-10). I shall not analyse these plot elements here, but will turn instead to these sequences' discursive content on the function of law enforcement during a public security crisis (in this case a major car accident). In this regard, four statements are made through visual and acoustic signs.

First, the police are again portrayed as professionals. Officers are shown securing the crash site in an organised fashion, speaking on their walky-talkies and directing the emergency vehicles (for example in NCNM 07-14, shots 02, 05 and 07; NCNM 07-17, shots 01, 03 and 04). In the first sequence, two officers are depicted questioning a witness with candid questions and diligently taking notes (shots 12 and 13). In the second sequence, the situation is directed by a police commissioner (who collaborates with manager Qiu Shi, which again asserts the 
fact that the male lead is a man of action who wields substantial authority; shot 08). This commissioner takes immediate and appropriate action when an additional complication occurs (a child with a heart problem has not been found and requires urgent medication). In this sense, verbal statements, gestures, the general set design and use of equipment, and the way in which the plot is developed, all contribute to the sense that the police are in charge of this hazardous situation, and that the law enforcement officials know what they are doing.

Second, the police are portrayed as being dedicated and helpful. Various shots in both sequences show police officers searching the surrounding for missing persons (for instance NCNM 07-14, shots 01, 02, 04 and 07; NCNM 07-17, shots 01, 25, 27, 28, 29 and 30) and helping victims of the accident out of the bus wreck (for example NCNM 07-14, shots 06, 09 and 11 ; NCNM $07-17$, shots $35,36,37,38$ and 39 ). What is particularly revealing here is that the sequences also feature medical personnel and fire-fighters (for example in NCNM 07-14, shots 01 and 04), but that shots of uniformed police clearly dominate: in the first sequence, every single shot features uniformed police; in the second more elaborate sequence, seventyseven per cent of all shots show police officers (Figure 56).

This is then also the third statement: the police are reacting to the accident with a massive amount of force. Not only are uniformed men and women omnipresent in both sequences, but the camera movements and angles also maximise the visual effect of their presence: numerous shots employ a camera crane to show birds-eye perspectives of the spectacle and move across the crowded scene (NCNM 07-14, shots 02, 05, 08, 13, 14, 17, 19, 21 and 22; NCNM 07-17, shots $07,14,18,32,38,41$ and 45). The significance is that the police are deploying a substantial contingent of men and women to solve this crisis and are hence taking what is de-facto a car crash very seriously. 


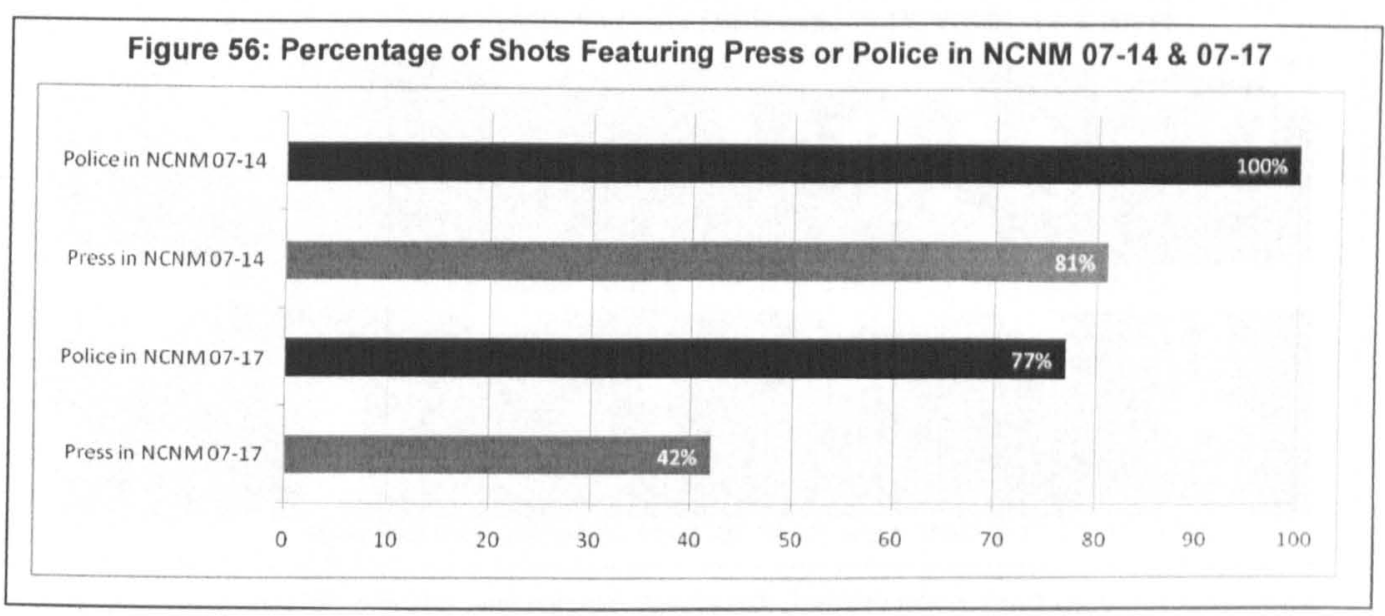

The final statement concerns the juxtaposition of the public security discourse with another discourse strand: the media discourse. Similar to the constant presence of law enforcement officials within the shots of these two sequences, the press are ever present. In the first of the two sequences, eighty-one per cent of all shots show journalists taking pictures or filming the proceedings; in the second sequence, forty-two per cent of the shots show members of the press reporting on the crisis (see both Figure 56 and Figure 57). Even in the shots that do not explicitly show photographers or cameramen, the presence of the media is nonetheless constantly signified by two signs: both sequences are acoustically underscored by the continuous clicking sound of cameras shooting photos, and repeatedly feature the visual sign of flash-lights illuminating the set. 


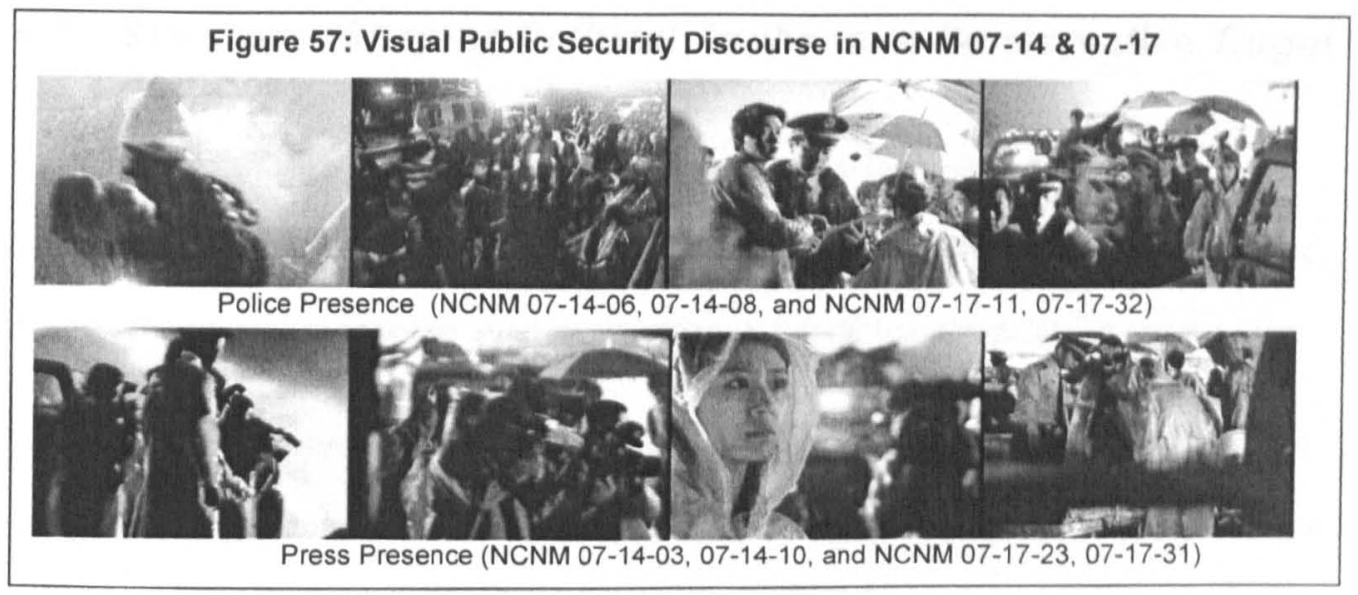

Through these techniques the impression is created that the crisis management is transparent and under the diligent scrutiny of the public. This notion is further strengthened by the fact that the press are allowed access to the crash site and the area in which the victims are treated by medics. There are a few shots that show police officers pushing journalists out of the way, but these actions are presented in a context that reinforces the authoritative and professional nature of the law enforcement personnel, and not in any way that might leave the impression that the press were unwelcomed at the site. To the contrary, the photographers and cameramen are presented as a constant background feature, suggesting that media scrutiny of law enforcement actions is a common-place. Note how the two series NCNM and GAJZ differ in this respect: whereas NCNM strongly makes use of the media discourse strand (thereby presenting the discursive position that the journalistic investigation and public dissemination of events is part of every-day life; see also NCNM 0105, 02-14, 10-04, 15-14 and 20-23), the main melody drama GAJZ does not contain comparable discourse fragments and only suggests verbally that the public is observing the police force's work. However, despite this discrepancy in presentation forms, the discursive statement made in both series concerning the public scrutiny of law enforcement activities is nonetheless the same (Figure 57):

- The police are diligently monitored by the people. 


\subsection{Summary: Shaping Political Truths for a Conservative Target Audience}

The above analysis has shown that even a communication form as seemingly trivial as television dramas substantially draws from and in turn contributes to political discourses, thereby shaping what concepts such as governance, the citizen, the police or crime mean.

In HWDD, the discourse on those who govern (the emperor) and those who are governed (the people) is presented as an amiable relationship between a fatherly ruler and his devoted subjects. The ruler Emperor Liu Che is presented as a humane figure who cares about his subordinates and the people. In so doing, the drama series combines the classical notion of the paternal ruler (though in a modern interpretation, with 'fatherly' here signifying kindness, not rigidity and strictness) with the Maoist concept of the state serving the people. At the same time the series shows the common people supporting their ruler despite abject poverty and immense suffering. The argument here is that such suffering is for a good cause: the expansion of the great Han nation. Considering that the target audience of such dramas coincides with the demographic group of the villagers shown in HWDD, it is likely that the creators of the series were hoping that the experience of suffering patriotically for the greatness of China would be one that their viewers could share.

This patriotic sentiment is also found in the discourse on security issues: the drama presents with much pathos the martial foreign security policy of the Han emperor as a necessary means to a great end: the expansion of the Han nation. Similarly, the failure of the Han envoy Zhang Qian to recruit foreign allies for the war against the barbaric Huns is reevaluated in the face of Zhang's other achievements. A case is made here for the peaceful coexistence of various ethnic groups, and for the harmonizing effect that trade has on the relations between such groups: Through its visuals, acoustics, and its narrative, the drama shows Zhang Qian as the person who established the first trade relations between the Han 
Chinese and their western neighbours, thereby bringing together these ethnically diverse peoples in a bustling and peaceful exchange of exotic goods. This achievement is then reinterpreted with much pathos as an achievement of the great Han nation, and is legitimized by the claim that historians across the world agree with this particular interpretation of the events.

A similar use of patriotism can be found in the modern crime drama GAJZ. In particular the security discourse in this main melody drama exhibits parallels to that of the historical drama HWDD, even though the latter deals with foreign security issues, while GAJZ is concerned with public security. However, GAJZ purveys a similarly militant notion with regard to security: the police are visually and acoustically presented in a way that constructs them as an army going to war against crime. At the same time, verbal statements throughout the series draw heavily from military vocabulary to re-enforce this point. Considering that the presentation of a huge, heavily armed and technologically advanced police force stands in no relation to the public security threats presented in the drama suggests that the overwhelming display of executive power is meant to inspire awe and a sense of patriotic pride in the viewer, while at the same time promoting the valour of the Chinese People's Police.

However, this is not the only parallel that the propaganda drama GAJZ shares with the historical series HWDD. It also contains strong references to the idea that the government serves the people. In addition, GAJZ presents a similar discourse concerning who the 'common people' are: elderly, often female, and on occasion disabled persons. It is of course no coincidence that this group should again largely overlap with the target audience of prime-time TV in China, and it can be argued that similar to the case of HWDD this is an attempt by the producers to encourage this particular demographic group to identify itself with the television drama. 
NCNM at first sight differs substantially from the other two series in the way it presents discourses on governance and security. How the state governs its subject and what security problems threaten the stability of society are side issues in the series. Instead, the programme focuses first and foremost on topics such as love relations and consumerism. It is in this respect revealing that the only one of the three drama series solely financed and created by private companies is also the one to focus primarily on the entertainment function of television, and not on propaganda or didactics. It is also striking how the series seems to be characterised by an almost complete absence of state authority.

However, a close analysis of this series nonetheless reveals political discourses at work, and it may at first sight seem surprising that essentially the same discursive statements concerning security issues are made here as in the other series. The police are portrayed as friendly, well-trained, technologically advanced professionals who serve the people by being ever watchful. Also, the idea that public servants answer to the public is relayed by a juxtaposition of the security discourse strand with the discourse strand on the media. The reason for this is that the depiction of good officials instils pride in the target audience of prime-time TV and compels viewers to 'tune in'.

While NCNM does not contain a similar militant or patriotic sentiment as HWDD or GAJZ, it is nevertheless noteworthy that the drama strongly features conservative gender themes. Discourse fragments on clichéd male-female relations and role functions were included here alongside more emancipated themes to provide consistently conservative conclusions to various plot complications. The reason for this is again the same one that fuels the repeated recourse to nationalist sentiments in the other two drama series: all three programs are tailored to a certain market that demands precisely such themes. 


\section{Conclusion: Chinese TV Discourses and the Factors of their Production}

This qualitative research project has demonstrated what types of political discourses are communicated through popular television and what factors generally lead to the production of such discourses. In this sense, my analysis answers a question that previous research has not adequately addressed: do popular Chinese entertainment formats such as TV dramas constitute a commercial enterprise or a political propaganda tool? Other authors have argued either that all content on central television is blatant propaganda which is commissioned and controlled by the Chinese leadership (Kramer, 2004; Yan, 2005), or that market reforms have liberalised the entertainment industry to such an extent that it is becoming increasingly difficult for the authorities to dictate content at all (Griffiths, 2005; Zhang et al., 2005). My work suggests that the problem is much more complex, and that its answer is situated somewhere between these two extremes.

My analysis has shown that television drama in China is created within an intricate system of political, cultural and economic constraints. One of the factors that influence the creation of drama content in the PRC is indeed the Chinese leadership. Through censorship guidelines and procedures, the government and the party continue to exert control over the system. It can therefore be argued that the Chinese authorities are controlling television content in order to assure the political hegemony of $\mathrm{CCP}$ cadres, and the above analysis does in fact show that such a motivation is partially responsible for the regulations that govern the broadcasting and production system in China. It is also partially responsible for the content of explicitly 'main melody' dramas such as GAJZ. This may at first sight lead to the misconception that all TV dramas are propaganda, particularly when considering that series from various genres and with different production backgrounds, such as GAJZ, HWDD, and NCNM, all draw from the same conservative notions of nationalism and gender. This then confirms Mark Harrison's observation that TV content in China is remarkably homogenous 
(2002). However, my research suggests that except for the particular case of the main melody programme, centrally televised drama content as a whole should not automatically be categorised as propaganda. This is evident on the one hand from the fact that China's elites criticise partially state-funded CCTV programmes such as HWDD as feudalistic trash; in fact such dramas are not commissioned by the authorities to promote the CCP's rule over China as some claim (Yan, 2005). Their content is instead influenced by more factors than merely state and party control. On the other hand, commercial programmes such as NCNM are entirely produced by private institutions and, as my conversations with production crew members who worked on that particular programme show, are not at all influenced by government or party directives; again, the content of such programmes is influenced by other factors.

This leads me to the conclusion that drama content in China is indeed highly ideological (meaning that it relays certain discursive positions through the conscious arrangement of signs in different modes), but that this ideological dimension does not stem from 'hard' state intervention. The question then is: why are Chinese television dramas so ideological. My research indicates that three factors are important in this regard.

First, political controls still apply to the Chinese entertainment sector, but they no longer directly dictate what is produced. In fact, the 'hard' censorship tools that formerly played a role in this system are no longer sufficient to control content production and distribution in the increasingly liberalised market. However, despite the fact that 'hard' controls have been loosened by market dynamics, a range of 'soft' controls still functions, thereby assuring that the Chinese television drama market remains highly rigid. These 'soft' controls (such as incentives for the production of certain content, mechanisms that inspire self-censorship, etc.) are not able to dictate the communication of specific propaganda statements, but they do function as a general framework or confinement within which drama content is produced. 
This is the reason why the general format of Chinese drama series has not yet converged with similar foreign formats: the various political restrictions only allow for a certain type of narrative (usually twenty episodes with an overarching storyline, which are produced and aired en-bloc), because other formats (such as the continuously produced soap opera that is common in other countries) would eventually render the few remaining forms of political control useless. This is then, in a sense, the 'outer boundary' of entertainment production in China.

Second, from a discourse-analytical perspective, it is important to realise that the Chinese explanations on how the broadcasting system works (and should work) are deeply rooted in production crew members' perceptions of China's social, economic and political problems, as well as in conceptions of how China's development should progress in the future in order to assure the population's welfare. It is further worth noting that Chinese intellectuals, professionals and government officials all genuinely believe that their assessments of what television content's relevance to Chinese society is, are true. In this sense, the tropes and metaphors used in the Chinese debate on media content create and enforce a reality in which government control is not only acceptable, but necessary. In particular the trope of social stability and economic progress has considerable constructivist power in this context, and defines how the Chinese elites view their responsibility towards the 'common people'. It would be interesting to conduct a survey to establish how far the economic development argument has been adopted by the average Chinese citizen in his or her everyday discourses, and to what extent the 'common people' accept the role that the elites have constructed for them. This question cannot be sufficiently answered in this thesis, but my hypothesis would be that the argument described above is in fact accepted by the often evoked 'masses' as much as by the elites. 
Third, the introduction of market reforms in China is a crucial aspect in the production of television entertainment. However, instead of displacing political controls as some observers have claimed, the liberalised market has introduced a whole range of new restrictions that in fact work in harmony with the old ones. These new restraints arise from a complex series of individual factors such as the audience rating system, producers' dependency on a limited range of distributors, Chinese broadcasters' profit-making strategies and target audience's viewing preferences. These factors influence each other and therefore create a system that constantly reinforces itself:

1. The television rating system assesses mainly urban households, and by default primarily measures the viewing habits and preferences of older, often uneducated or unemployed, mostly female urban viewers;

2. The broadcasting system relies on this data to sell advertising slots, and hence specifically airs television content in a way that will satisfy this viewer groups' preferences (i.e. broadcasting a full drama series consecutively, two episodes per day, until the drama's conclusion);

3. The production companies, aiming to sell their products to the broadcasters, primarily create drama content that fits this format (i.e. dramas with a closed twenty episode narrative, featuring the discourses and modes of expressions that will attract an older urban female audience);

4. The general offer of television content excludes younger, more educated viewers and instead appeals again to the same typical drama audience, who in turn comment favourably on the TV content through the various channels of communication, tune in when new drama series are shown, and are consequently again measured by the audience rating system;

5. The cycle therefore begins anew. 
It is thus at the cross-section of these three institutional fields (the political, the cultural and the economic) that the creation of television content is negotiated in the PRC. The creative environment that arises from this condition offers little room for the development of innovative contents or counter-hegemonic discourses. Instead, this environment facilitates the construction of conservative and often nationalist discourses in drama series. It can therefore be argued that even if China abolished the last of its 'hard' censorship mechanisms, this would have little effect on the homogeneity of Chinese drama discourses.

Aside from clarifying how drama content is produced in China, this research project has also contributed to the theoretical debate on how visual communication works. My analysis has shown that popular entertainment formats such as television drama series function as complex mass-communication practices, and that these practices are capable of purveying political meaning. The scientific examination of such practices calls for a broad methodological approach that takes into account not only the factors that lead to the production of content, but also the various levels at which this content communicates meaning. I have presented such an approach in Chapter 2: by combining a critical discourse analysis perspective with a visual analytical methodology grounded in semiotic theory, this approach enables us to identify the various 'signs' (or markers of meaning) that function within the different modes of such communication practices, to isolate what 'discourse fragments' (that is: truth claims) these signs create, and to analyse how a variety of factors contributed to the formulation of such statements. This approach has considerable explanatory value when it comes to the analysis of visual formats such as television dramas, but it also offers a theoretical basis and a range of tools that lend themselves to research into other mass-communication practices. The analysis presented here suggests that such projects would offer valuable insights into how political discourses are communicated throughout a wide variety of visual contexts. In this sense, the present research opens up various 
opportunities for future projects in three different disciplines: Chinese studies, political science, and communication or media studies.

With regard to Chinese studies, visual media content is a particularly relevant research field that has received little attention from scholars in the West: my interviews with Chinese media experts have shown that the Chinese leadership and the professionals who create television content both attribute strong political and social power to visual formats such as mass-communicated images. The use of these visual forms is tied to a conscious effort by those who produce them to guide public opinion and educate the 'common people'. In this sense, all visual practices that communicate to these 'masses' (and not only the televised image) need to be understood as tools that are aimed at instructing and educating the recipients, and therefore as social forces that create meaning throughout Chinese society. Researchers in Chinese Studies need to realise the importance of visual communication in the PRC, and should not neglect the political and social impact of a wide variety of communication practices. The following issues promise to pose particularly promising future research questions:

- While this research project has answered the question of how political discourses are constructed in popular Chinese television drama series, it nevertheless had to exclude a number of issues. The analysis examined only two political discourse strands (governance and security) in three particular television drama series aired on national television during a specific time interval (between 2003 and 2005). Consequently, this research has a limited focus. While this limitation does not make the research results any less valid (the drama series examined here are each representative models of their respective genre, were all highly popular with the Chinese audiences, and were produced in different institutional settings), the question still remains how political discourses are portrayed in other TV drama genres (for instance family dramas, kung 
fu series, or "pink" programmes), in particular local settings (such as the market segment that stations such as BTV, STV, or OTV cover), and at other times in PRC history (for example subsequent to major events such as Hong Kong's return to the mainland, China's accession to the WTO, or the Olympic Games). It would be of particular interest to establish how current events influence the formulation of discourse fragments; a question that the present thesis did not address. At the same time, an analysis of additional political issues (for instance welfare) in these and other drama series is called for; such analyses promise to further contribute to our understanding of what social and political 'truths' such popular entertainment formats communicate.

- It should also be pointed out that this analysis has approached visual material from the production side and the product side. It has not examined the effect side of television dramas in China. Quantitative surveys by companies such as AGB Nielsen or CSM that attempt to provide reliable data on who watches what on Chinese television are faced with substantial demographic challenges in a nation as large as the PRC, which leads them to fall back on insufficient statistical samples. This then raises the question of whether representative data can at this point be collected in such a diverse environment. Qualitative approaches to the field of viewer analysis offer a viable alternative, but past research on how Chinese audiences watch television and what relevance TV entertainment truly plays in their lives are today either outdated (Lull, 1991), lack a comprehensive research methodology (Kramer, 2006), or use a nonrepresentative sampling base (ibid.; $\mathrm{Li}, 2007$ ). The challenge for future projects will be to address these problems and provide a coherent and representative answer to the question of who watches what in China, and why. 
- Authors such as Zhao Yuezhi (1998) have presented insightful works on Chinese news broadcasts. Such research should be augmented with visual analyses of Chinese programmes such as CCTV's daily evening news or of CCTV news magazines such as Dongfang shikong 东方时空 or Jiaodian fangtan 焦点访谈.

- Visual political analyses are also needed of other television formats, for instance of talk shows such as Shihua shishuo 实话实说, or of educational programmes such as CCTV's lecture room series on historical and philosophical topics (Baijia jiangtan 百家 讲坛).

- Staged events in China, for instance opening ceremonies for events such as the Beijing Olympics or the Shanghai Expo, have not been approached from a visual political communication perspective. The semiotic and discourse theoretical framework used in this present project offers the tools for an examination of how various signs are arranged in such a mass-communicated mega-event to communicate political meaning.

- Finally, the semiotic framework used here could easily be extended to the analysis of architectural projects and monuments. Such research could focus either on small artistically and politically relevant constructions such as specific hero memorials, but could also analyse the visual political impact of single buildings such as the new Beijing airport terminal, the Beijing Olympic Stadium, or the new CCTV office tower. It would further be a promising endeavour to examine the political relevance of large urban-planning projects from a communication perspective, for instance the arrangement of the various World Exposition sites along the Pujiang River or the new deep-see harbour near Shanghai. 
In the field of media studies, approaches such as the one adopted in this thesis also promises to yield new insights into more general communication practices. The following gaps in the current literature need to be filled:

- Comparative research is needed in order to establish how the production and distribution process of Chinese dramas differs from similar procedures in other societies, and how such processes tie into global trade patterns. Previous comparative works, especially on Latin-American formats and US entertainment programmes, indicate the direction that such potential future research could take (Hong, 1998; Lopez, 1995; Martín-Barbero, 1995).

- Previous studies of media discourses that mostly analysed verbal or textual aspects of communication should extend their focus to visual elements, such as the photos used in newspapers or the selection and montage of visual material in Western news broadcasts. Works on visual culture by author's such as Gunther Kress and Theo van Leeuwen (2001), Myra MacDonald (2003), or Nicholas Mirzoeff (2005) are steps in the right direction, but they would further profit form detailed visual fine-analyses like the one presented here.

With regard to political science, the present project also raises a number of new questions, particularly concerning the problem of how a society under increased liberalization, internationalization and commercialization pressures can still be effectively governed. This issue is a relatively recent yet nonetheless pressing concern in political science (Gamble, 2000; Jessop, 1997 \& 1998; Stoker, 1998). China is a particularly interesting case in point: the market forces that have been unleashed in the PRC have compelled the leadership to revise their authoritative top-down way of governing. The regulation of the TV drama market shows that new, soft mechanisms of control are largely replacing the former hard regulatory methods. Is China then moving towards a new system of governance? And how does this 
new approach compare to governance concepts in Western societies? Also: should the move towards softer regulatory practices be regarded as a chance to increase pluralism and participation, or have the former mechanisms merely been replaced with a new set of controls that now follows a capitalist rather than socialist logic? Such questions need to be answered through in-depth analyses of China's new governance methods in a variety of policy fields:

- Media and information policy, particularly the issue of content control;

- Economic policy, particularly urban development projects;

- Public security, particularly the management of public conduct.

These are examples of questions that future research should address. This analysis is a first step in this direction. It has shown that entertainment formats in the PRC are an important method of communicating political meaning and contributing to the construction of social and political reality in China. In this sense, mass-communication formats deserve attention from researchers in the social sciences and in Chinese Studies alike. They need to be understood no longer merely as texts that function primarily through verbal expressions, but as complex communication practices that relay their content in various modes. My research has shown that an analysis that takes these modes into account has strong explanatory power and helps us to understand how visual communication works in the PRC. It shows how such communication practices are shaped both by direct policy interventions and by the personal perceptions of those involved in them, and it further shows how political such processes truly are. 


\section{Bibliography}

ALLEN, ROBERT C. (1992a), "Audience-Oriented Criticism and Television", in: ALLEN, ROBERT C. (ed.), Channels of Discourse, Reassembled : television and contemporary criticism, second edition, 2nd ed., London: Routledge, 101-137.

ALLEN, ROBERT C. (ed.) (1992b), Channels of Discourse, Reassembled : television and contemporary criticism, second edition, 2nd ed., London: Routledge.

ALLEN, ROBERT C. (ed.) (1995), To Be Continued... Soap Operas Around the World, London: Routledge.

BALDWIN, KATE (1995), "Montezuma's Revenge : Reading Los Ricos También Lloran in Russia", in: ALLEN, ROBERT C. (ed.), To Be Continued... : Soap Operas Around the World, London: Routledge, 285-300.

BARKER, CHRIS (1997), Global Television : An Introduction, Oxford: Blackwell Publishing Ltd.

BARTHES, ROLAND (1977), "Rhetoric of the Image", in: HEATH, STEPHEN (ed.), Roland Barthes - Image, Music, Text, 11. ed., London: Fontana Press, 32-51.

BBC (2004a), China Opens TV to Foreign Firms, London: BBC News Online, http://news.bbc.co.uk/go/pr/fr/-/1/hi/business/4022273.stm [18.11.04].

BBC (2004b), Friends 'Too Sexy' for Chinese TV, London: BBC News Online, http://news.bbc.co.uk/go/pr/fr/-/1/hi/entertainment/tv_and_radio/3410231.stm [19.01.04].

BBC (2006a), China Defends Internet Regulation, London: BBC News Online, http://news.bbc.co.uk/go/pr/fr/-/1/hi/world/asia-pacific/4715044.stm [15.02.06].

BBC (2006b), China Media Blank Out Hu Protest, London: BBC News Online, http://news.bbc.co.uk/go/pr/fr/-/1/hi/world/asia-pacific/4930054.stm [21.04.06].

BBC (2006c), Chinese Slap Ban on TV Cartoons, London: BBC News Online, http://news.bbc.co.uk/go/pr/fr/-/1/hi/entertainment/4743414.stm [23.02.06].

BORSTNAR, NIELS, PABST, ECKHARD \& WULFF, HANS JÜRGEN (2002), Einführung in die Film- und Fernsehwissenschaft (Introduction to Film-and Television-Studies), Konstanz: UVK Verlagsgesellschaft mbH.

BRUNNER, OTTO, CONZE, WERNER \& KOSELLECK, REINHART (eds.), (2004), Geschichtliche Grundbegriffe : historisches Lexikon zur politisch-sozialen Sprache in Deutschland (Basic Historical Terms : Historical Lexicon of Political-Social Language in Germany), Stuttgart: Klett-Cotta.

BRUNSDON, CHARLOTTE (1995), "The Role of Soap Opera in the Developement of Feminist Television Scholarship", in: ALLEN, ROBERT C. (ed.), To Be Continued... : Soap Operas Around the World, London: Routledge, 49-65.

"Censorship" (2005), World Encyclopedia, Oxford: Oxford University Press. 
CERNY, PHILIP G. (1996), "What Next for the State?" in: KOFMAN, ELEONORE \& YOUNGS, GILLIAN (eds.), Globalization : Theory and Practice, London: Pinter, 123-137.

CHAMBERS, SIMONE \& KOPSTEIN, JEFFREY (2008), "Civil Society and the State", in: DRYZEK, JOHN S., HONIG, BONNIE \& PHILLIPS, ANNE (eds.), The Oxford Handbook of Political Theory, Oxford \& New York: Oxford University Press, 363-381.

CHAN, JOSEPH MAN (2003), "Administrative Boundaries and Media Marketization : A Comparitative Analysis of the Newspaper, TV and Internet Markets in China", in: LEE, CHIN-CHUAN (ed.), Chinese Media, Global Contexts, London: RoutledgeCurzon, 159-176.

CHEN, XIAOCHUN (陈晓春) \& ZHANG, HONG (张宏) (2005)，电视剧制片管理 (TV Drama Production Management), Beijing: 北京大学出版社 (Beijing daxue chubanshe).

CHOULIARAKI, LILIE \& FAIRCLOUGH, NORMAN (1999), Discourse in Late Modernity Rethinking Critical Discourse Analysis, Edinburgh: Edinburgh University Press.

CONDRY, JOHN C. (1989), The Psychology of Television, Hillsdale NJ: Lawrence Erlbam Associates.

CORNELIUS, SHEILA \& SMITH, IAN HAYDN (2002), New Chinese Cinema : Challenging Representations, London: Wallflower.

CRAIG, TIMOTHY J. \& KING, RICHARD (eds.), (2002), Global Goes Local : Popular Culture in Asia, Vancouver: UBC Press.

CSM (2005)，中国电视剧市场报告2005-2006 (Report on the Chinese Television Drama Market 2005-2006), Shanghai: CSM, http://www.csm.com.cn/news/050613.html [17.05.06].

CUI, SHUQIN (2005), "Working from the Margins : Urban Cinema and Independent Directors in Contemporary China", in: LU, SHELDON H. \& YEH, EMILIE YUEH-YU (eds.), Chinese Language Film : Historiography, Poetics; Politics, Honolulu: University of Hawai'i Press, 96-119.

CURTIN, MICHAEL (2003), "The Future of Chinese Cinema : Some Lessons from Hong Kong and Taiwan", in: LEE, CHIN-CHUAN (ed.), Chinese Media, Global Contexts, London: RoutledgeCurzon, 237-256.

DAI, QING (緎清) (2004)，电规剧审美文化研究 (Aesthetic and Cultural Studies of TV Series), Beijing: 中国传媒大学出版社 (Zhongguo chuanmei daxue chubanshe).

DONALD, STEPHANIE HEMELRYK \& KEANE, MICHAEL (2002), "Media in China : New Convergences, New Approaches", in: DONALD, STEPHANIE HEMELRYK, KEANE, MICHAEL \& HONG, YIN (eds.), Media in China - Consumption, Content and Crisis, London / New York: RoutledgeCourzon, 3-17.

DONALD, STEPHANIE HEMELRYK, KEANE, MICHAEL \& HONG, YIN (eds.), (2002), Media in China : Consumption, Content and Crisis, London / New York: RoutledgeCourzon. 
DRECHSEL, ROBERT E. (2005), "Censorship", in: HALL, KERMIT L. (ed.), The Oxford Companion to the Supreme Court of the United States, Oxford: Oxford University Press.

DREYFUS, HUBERT L. \& RABINOW, PAUL (1982), Michel Foucault - Beyond Structuralism and Hermeneutics, Hertfordshire: Harvester Wheatsheaf.

EBERSTEIN, BERND (forthcoming), "Theatre", Brill's China Encyclopaedia, Leiden: Koninklijke Brill.

ECO, UMBERTO (1979), A Theory of Semiotics, Bloomington: Indiana University Press.

EDWARDS, DEREK, ASHMORE, MALCOLM \& POTTER, JONATHAN (1995), "Death and Furniture : The Rhetoric, Politics and Theology of Bottom Line Arguments Against Relativism", History of the Human Sciences 2: 25-49.

ELLENIUS, ALLAN (ed.) (1998), Iconography, Propaganda, and Legitimation, Oxford: Clarendon.

EVANS, GRAHAM \& NEWNHAM, JEFFREY (1998), Dictionary of International Relations, London et al.: Penguin Books.

FAIRCLOUGH, NORMAN (1995), Critical Discourse Analysis : The Critical Study of Language, Harlow: Pearson Education Limited.

FAULSTICH, WERNER (2002), Grundkurs Filmanalyse (Basic Course in Film Analysis), Munich: Wilhelm Fink Verlag GmbH.

FEUILHERADE, PETER (2005), China 'Ripe' for Media Explosion, London: BBC News Online, http://news.bbc.co.uk/go/pr/fr/-/1/hi/technology/4341413.stm [13.03.05].

FISKE, JOHN (1992), "British Cultural Studies and Television", in: ALLEN, ROBERT C. (ed.), Channels of Discourse, Reassembled : television and contemporary criticism, second edition, 2nd ed., London: Routledge, 284-326.

FLITTERMAN-LEWIS, SANDY (1992), "Psychoanalysis, Film, and Television", in: ALLEN, ROBERT C. (ed.), Channels of Discourse, Reassembled : television and contemporary criticism, second edition, 2nd ed., London: Routledge, 203-246.

FOUCAULT, MICHEL (1990), The History of Sexuality - An Introduction (Volume 1), New York: Vintage Books.

FOUCAULT, MICHEL (1995), Discipline \& Punish: The Birth of the Prison, New York: Vintage Books.

FOUCAULT, MICHEL (2005a), Archaeology of Knowledge, 4th ed., London: Routledge.

FOUCAULT, MICHEL (2005b), The Order of Things: An Archaeology of the Human Sciences, 5th ed., London: Routledge. 
FROSCHAUER, ULRIKE \& LUEGER, MANFRED (2003), Das qualitative Interview : Zur Praxis interpretativer Analyse sozialer Systeme (The Qualitative Interview : On the Practical Interpretatory Analysis of Social Systems), Vienna: WUV-Universitätsverlag.

FUNG, ANTHONY \& MA, ERIC (2002), "'Satelite Modernity': Four Modes of Televisiual Imagination in the Disjunctive Socio-Mediascapfe of Guangzhou", in: DONALD, STEPHANIE HEMELRYK, KEANE, MICHAEL \& HONG, YIN (eds.), Media in China - Consumption, Content and Crisis, London / New York: RoutledgeCourzon, 67-79.

FUQUA, JOY V. (1995), "'There's a Queer in my Soap!" : The Homophobia/ AIDS Story-Line of One Life to Live", in: ALLAN, STUART (ed.), To Be Continued... Soap Operas Around the World, London: Routledge, 199-212.

GAMBLE, ANDREW (2000), "Economic Governance", in: PIERRE, JON (ed.), Debating Governance - Authority, Steering, and Democracy London \& New York: Oxford University Press, 110-137.

GAMBLE, ANDREW (2006), "The European Disunion", The British Jourmal of Politics and International Relations 8: 34-249.

GERAGHTY, CHRISTINE (1995), "Social Issues and Realist Soaps : A Study of British Soaps in the 1980s/1990s", in: ALLAN, STUART (ed.), To Be Continued... Soap Operas Around the World, London: Routledge, 66-80.

GIDDENS, ANTHONY (1994), Beyond Left and Right - The Future of Radical Politics, Cambridge: Blackwell.

GOLDAIM (2002)，蓝印户口申报条件 (Conditions for Blue Card Hukou Applications), Shanghai: 上海金蓝图创业网 (Shanghai goldaim.com),

http://www.goldaim.com/lanyinhukoul.htm [30.06.06].

GOMBRICH, E. H. (1980), "Standards of Truth : The Arrested Image and the Moving Eye", in: MITCHEL, W. J. THOMAS (ed.), The Language of Images, 4th ed., Chicago: The University of Chicago, 181-217.

GRIFFITHS, ALISON (1995), "National and Cultural Identity in a Welsh-Language Soap Opera", in: ALLAN, STUART (ed.), To Be Continued... Soap Operas Around the World, London: Routledge, 81-97.

GRIFFITHS, DAN (2005), China's Breakneck Media Revolution, London: BBC News Online, http://news.bbc.co.uk/go/pr/fr/-/1/hi/world/asia-pacific/4156458.stm [19.08.05].

HAGEDORN, ROGER (1995), "Doubtless to be Continued : A Brief History of Serial Narrative", in: ALLEN, ROBERT C. (ed.), To Be Continued... : Soap Operas Around the World, London: Routledge, 27-48.

HALL, STUART; (ed.) (1980), Culture, Media, Language : Working Papers in Cultural Studies, 1972-79, London: HarperCollins. 
HARRISON, MARK (2002), "Satellite and Cable Platforms: Development and Content", in: DONALD, STEPHANIE HEMELRYK; KEANE, MICHAEL; HONG, YIN (ed.), Media in China - Consumption, Content and Crisis, London / New York: RoutledgeCourzon, 167-178.

HE, ZHOU (2003), "How do the Chinese Media Reduce Organizational Incongruence? Bureaucratic Capitalism in the Name of Communism", in: LEE, CHIN-CHUAN (ed.), Chinese Media, Global Contexts, London: RoutledgeCurzon, 196-214.

HEBERER, THOMAS (forthcoming), "Participation", Brill's Encyclopaedia of China, Leiden: Koninklijke Brill.

HEIMER, MARIA \& THØGERSEN, STIG (2005), Doing Fieldwork in China, Honolulu HI: University of Hawaii Press.

HOFFMANN, WILHELM (ed.) (1999), Die Sichtbarkeit der Macht. Theoretische und empirische Untersuchungen zur visuellen Politik. (The Visibility of Power. Theoretical and Empirical Analyses Concerning Visual Politics.), Baden-Baden: Nomos Verlagsgesellschaft.

HOLBIG, HEIKE (2005), "Internetkontrolle - jünste Entwicklungen (Internet Control Recent Developments)", China aktuell 59-60.

HOLBIG, HEIKE (2006), "Beijinger Boulevardzeitung jułngstes Opfer staatlicher Pressezensur (Beijing Tabloid Most Recent Victim of Chinese Press Censorship)", China aktuell 111113.

HONG, JUNHAO (1998), The Internationalization of Television in China : The Evolution of Ideology, Society, and Media Since the Reform, Westport CT: Praeger Publishers.

HONG, YIN (2002), "Meaning, Production, Consumption : The History and Reality of Television Drama in China", in: DONALD, STEPHANIE HEMELRYK, KEANE, MICHAEL \& HONG, YIN (eds.), Media in China - Consumption, Content and Crisis, London / New York: RoutledgeCourzon, 28-39.

HOOPES, JAMES (1991), "Peirce on Signs - Introduction", in: HOOPES, JAMES (ed.), Peirce on Signs - Writings on Semiotic by Charles Sanders Peirce, Chapel Hill \& London: The University of North Carolina Press, 1-13.

HOWARTH, DAVID (2000), Discourse, Buckingham \& Philadelphia: Open University Press.

HU, MEI (胡玫) (2005)，"汉武大帝 (The Great Emperor Wu of the Han Dynasty; HWDD)", 58 episodes, People's Republic of China: CCTV Literature and Art Department (中央电 视台文艺中心).

HUANG, KUANGYU (黄匡宇) (ed.) (2005)，当代电视陡桭制作数程 (Course in Contemporary Television Filming), Shanghai: 复旦大学出版社 (Fudan daxue chubanshe).

HUANG, YIFENG (黄一峰), TAO, TAO (陶涛), LI, MINGTAO (本铭輗) \& WANG, NAN (王 楠) (2006)，制片管理 (Film Making Management)，Beijing: 中国电影出版社 (Zhongguo dianying chubanshe). 
HUNTINGTON, SAMUEL P. (1996), The Clash of Civilizations and the Remaking of World Order New York: Simon \& Schuster.

INTINTOLI, MICHAEL JAMES (1984), Taking Soaps Seriously : The World of Guiding Light, New York: Praeger Publishers.

JÄGER, SIEGFRIED (2004), Kritische Diskursanalyse. Eine Einführung. (Discourse Analysis. An Introduction.), 4th unabr. ed., Münster: UNRAST-Verlag.

JESSOP, BOB (1997), "Capitalism and its Future : Remarks on Regulation, Government and Governance", Review of International Political Economy 4/3: 561-581.

JESSOP, BOB (1998), "The Rise of Governance and the Risks of Failure : The Case of Economic Development", International Social Science Journal 155: 29-45.

JIN, TAO (金蹈) (2003), "公安局长二 (The Commissioner - Part 2; GAJZ)", 20 episodes, People's Republic of China: Golden Shield Centre of the Public Security Ministry (公 安部金盾影视文化中心).

KAPLAN, E. ANN (1992), "Feminist Criticism and Television", in: ALLEN, ROBERT C. (ed.), Channels of Discourse, Reassembled : television and contemporary criticism, second edition, 2nd ed., London: Routledge, 247-283.

KATZ, STEVEN D. (1991), Film Directing Shot by Shot : Visualizing from Concept to Screen, Studio City: Michael Wiese Productions.

KEANE, MICHAEL (2002a), "Send in the Clones: Television Formats and Content Creation in the People's Republic of China", in: DONALD, STEPHANIE HEMELRYK; KEANE, MICHAEL; HONG, YIN (ed.), Media in China - Consumption, Content and Crisis, London / New York: RoutledgeCourzon, 80-90.

KEANE, MICHAEL (2002b), "Television Drama in China : Engineering Souls for the Market", in: CRAIG, TIMOTHY J.; KING, RICHARD (ed.), Global Goes Local - Popular Culture in Asia, Vancouver: UBC Press, 120-137.

KEANE, MICHAEL (2005), "Television Drama in China : Remaking the Market", Media International Australia (Culture and Policy) 115: 82-93.

KELLNER, DOUGLAS (1995), Media Culture : Cultural Studies, Identity and Politics Between the Modern and the Postmodern, London: Routledge.

KORTE, HELMUT (1999), "Filminterpretation - Filmanalyse (Film Interpretation - Film Analysis)", in: HOFFMANN, WILHELM (ed.), Die Sichtbarkeit der Macht. Theoretische und empirische Untersuchungen zur visuellen Politik. (The Visibility of Power. Theoretical and Empirical Analyses Concerning Visual Politics.), Baden-Baden: Nomos Verlagsgesellschaft, 76-90.

KOZLOFF, SARAH (1992), "Narrative Theory and Television", in: ALLEN, ROBERT C. (ed.), Channels of Discourse, Reassembled : television and contemporary criticism, second edition, 2nd ed., London: Routledge, 67-100. 
KRAMER, STEFAN (2004), Vom Eigenen und Fremden. Fernsehen und kulturelles Selbstoerständnis in der Volksrepublik China. (Concerming the local and the global. Television and cultural self-conception in the People's Republic of China.), Bielefeld: transcript Verlag.

KRAMER, STEFAN (2006), Das chinesische Fernsehpublikum. Zur Rezeption und Reproduktion eines neuen Mediums (The Chinese Television Audience. Concerning the Reception and Reproduction of a New Medium), Bielefeld: transcript Verlag.

KRESS, GUNTHER \& VAN LEEUWEN, THEO (2001), Multimodal discourse - The modes and media of contemporary communication, London: Arnold.

KREUTZNER, GABRIELE \& SEITER, ELLEN (1995), "Not all 'Soaps' are Created Equal : Toward a Cross-Cultural Criticism of Television Serials", in: ALLEN, ROBERT C. (ed.), To Be Continued... : Soap Operas Around the World, London: Routledge, 234-255.

LEE, CHIN-CHUAN (ed.) (2003), Chinese Media, Global Contexts, London: RoutledgeCurzon.

LEE, CHIN-CHUAN (2003), "The Global and the National of the Chinese Media : Discourses, Market, Technology, and Ideology", in: LEE, CHIN-CHUAN (ed.), Chinese Media, Global Contexts, London: RoutledgeCurzon, 1-31.

LEGGEWIE, CLAUS (ed.) (1994), Wozu Politikwissenschaft? Über das Neue in der Politik. (Why Political Science? On Novelties in Politics.) Darmstadt: Wissenschaftliche Buchgesellschaft.

LI, JINGCHENG (李京盛) (ed.) (2007)，中国电视剧年度发展报告 (Annual Development Report on Chinese Television Drama), Beijing: 中国传媒大学出版杜 (Zhongguo chuanmei daxue chubanshe).

LIU, LIWEN (刘丽文) (2005)，历史剧的女性主义推评 (Feminist Critique of Historical Television Dramas), Beijing: 中国传媒大学出版社 (Zhongguo chuanmei daxue chubanshe).

LOPEZ, ANA M. (1995), "Our Welcomed Guests : Telenovelas in Latin America", in: ALLAN, STUART (ed.), To Be Continued... Soap Operas Around the World, London: Routledge, 256-275.

LU, HAIBO (路海波) \& LI, MENGXUE (本梦学) (eds.), (2006), 制片管理 (Production Management), Beijing: 中国电影出版社 (Zhongguo dianying chubanshe).

LU, SHELDON H. (2005), "Chinese Film Culture at the End of the Twentieth Century : The Case of Not One Less by Zhang Yimou", in: LU, SHELDON H. \& YEH, EMILIE YUEH-YU (eds.), Chinese Language Film : Historiography, Poetics, Politics, Honolulu: University of Hawai'i Press, 120-137.

LU, SHELDON H. \& YEH, EMILIE YUEH-YU (eds.), (2005), Chinese Language Film : Historiography, Poetics, Politics, Honolulu: University of Hawai'i Press.

LU, YE (陆眊) \& ZHAO, MIN (赵民) (eds.)，(2002)，当代广播电规㩯论 (Introduction to Contemporary Radio and Television Broadcasting), Shanghai: 夏旦大学出版社 (Fudan daxue chubanshe). 
LULL, JAMES (1991), China Tumed On : Television, Reform and Resistance, London / New York: Routledge.

MACDONALD, MYRA (2003), Exploring Media Discourse, Oxan: Oxford University Press US.

MARSHALL, T. H. (1975), Social Policy in the Twentieth Century, 4th ed., London: Hutchinson.

MARTÍN-BARBERO, JESÚS (1995), "Memory and Form in the Latin American Soap Opera", in: ALLEN, ROBERT C. (ed.), To Be Continued... : Soap Operas Around the World, London: Routledge, 276-284.

MAYRING, PHILIPP (2002), Einführung in die Qualitative Sozialforschung - Eine Anleitung zu qualitativem Denken (Introduction to Qualitative Social Science Research - Instruction Manual to Qualitative Thinking), 5th ed., Weinheim / Basel: Beltz Verlag.

MCCORMICK, BARRETT L. (2004), "Censorship and Accountability in Chinese Media", Cambridge MA.

MCCORMICK, BARRETT L. \& LIU, QING (2003), "Globalization and the Chinese Media : Technologies, Content, Commerce and the Prospects for the Public Sphere", in: LEE, CHIN-CHUAN (ed.), Chinese Media, Global Contexts, London: RoutledgeCurzon, 139158.

MCLEAN, IAIN \& MCMILLAN, ALISTAIR (eds.), (2003), The Concise Oxford Dictionary of Politics, 2nd ed., Oxford \& New York: Oxford University Press.

MII (2005), 信息产业部职素 (The Responsibilities of the Ministry of Information Industry), Beijing: MII, http://www.mii.gov.cn/col/col22/index.html [15.05.06].

MIKOS, LOTHAR (2003), Film- und Fernsehanalyse (Film- and Television-Analysis), Konstanz: UVK Verlagsgesellschaft mbH.

MIRZOEFF, NICHOLAS (ed.) (2005), The Visual Culture Reader, London: Routledge.

MÜLLER, MARION G. (1997a), "Politik mit bewegten Bildern. Wahrnehmung und Wirklichkeit der Wahlwerbung. (Politics in Moving Images. Reception and Reality of Election Advertisements)", Sowi - Sozialwissenschaftliche Informationen 3: 239-247.

MÜLLER, MARION G. (1997b), "Seht mich, liebt mich, wählt mich! Wahlkampf in der ikonischen Offentlichkeit am Beispiel des Bundestagswahlkampfes 1998 (See Me, Love Me, Elect Me! Election Campaigns in the Iconic Public Sphere - the Example of the German General Election 1998)", in: WINTERHOFF-SPURK, PETER \& JÄCKEL, MICHAEL (eds.), Politische Eliten in der Mediengesellschaft (Political Elites in Media Society), Munchen: Verlag Reinhard Fischer, 121-138.

MÜLlER, MARION G. (1999), "Politische Vision (Political Vision)", in: HOFFMANN, WILHELM (ed.), Die Sichtbarkeit der Macht. Theoretische und empirische Untersuchungen zur visuellen Politik. (The Visibility of Power. Theoretical and Empirical Analyses Concerning Visual Politics.), Baden-Baden: Nomos Verlagsgesellschaft, 15-27. 
MÜLLER, MARION G. (2003), Grundlagen der visuellen Kommunikation (Basics of Visual Communication), Konstanz: UVK Verlagsgesellschaft mbH.

MUNRO, DONALD J. (1969), The Concept of Man in Early China, Stanford: Stanford University Press.

PAN, ZHONGDANG \& LU, YE (2003), "Localizing Professionalism : Discoursive Practices in China's Media Reforms", in: LEE, CHIN-CHUAN (ed.), Chinese Media, Global Contexts, London: RoutledgeCurzon, 215-236.

PANOFSKY, ERWIN (1987), "Zum Problem der Beschreibung und Inhaltsdeutung von Werken der Bildenen Kunst (Concerning the Problem of Describing and Interpreting Works of Art)", in: KAEMMERLING, EKKEHARD (ed.), Ikonographie und Ikonologie (Iconography and Iconology), Berlin: Verlag Bruno Hessling, 185-206.

PEIRCE, CHARLES SANDERS (1991a), "On the Nature of Signs", in: HOOPES, JAMES (ed.), Peirce on Signs - Writings on Semiotic by Charles Sanders Peirce, Chapel Hill \& London: The University of North Carolina Press, 141-143.

PEIRCE, CHARLES SANDERS (1991b), "Sign", in: HOOPES, JAMES (ed.), Peirce on Signs Writings on Semiotic by Charles Sanders Peirce, Chapel Hill \& London: The University of North Carolina Press, 239-240.

PHILLIPS, WILLIAM H. (2002), Film : An Introduction, 2nd ed. ed., Boston / New York: Palgrave.

PHILO, GREG (2007), "Can Discourse Analysis Successfully Explain the Content of Media and Journalistic Practice", Journalism Studies 8/2: 175-196.

POLUMBAUM, JUDY (2003), "Capturing the Flame : Aspirations and Representations of Beijing's 2008 Olympics", in: LEE, CHIN-CHUAN (ed.), Chinese Media, Global Contexts, London: RoutledgeCurzon, 57-75.

POTTER, JONATHAN (2005), Representing Reality - Discourse, Rhetoric and Social Construction, London: SAGE.

PREUCEL, ROBERT W. (2006), Archaeological Semiotics, Oxford et al.: Blackwell Publishing Ltd.

PYE, LUCIAN W. (forthcoming), "Political Culture", Brill's Encyclopaedia of China, Leiden: Koninklijke Brill.

REDL, ANKE \& SIMONS, ROWAN (2002), "Chinese Media - One Channel, Two Systems", in: DONALD, STEPHANIE HEMELRYK, KEANE, MICHAEL \& HONG, YIN (eds.), Media in China - Consumption, Content and Crisis, London / New York:

RoutledgeCourzon, 18-27.

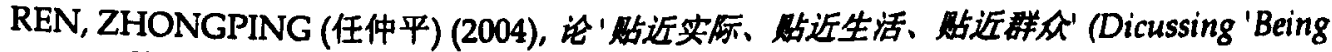
Close to Reality, Close to Life, and Close to the Masses'), Beijing: RMRB, http://news.xinhuanet.com/newmedia/2004-12/16/content_2344978.htm [28.01.08]. 
ROFEL, LISA (1995), "The Melodrama of National Identity in Post-Tiananmen China", in: ALLEN, ROBERT C. (ed.), To Be Continued... : Soap Operas Around the World, London: Routledge, 301-320.

ROSEN, STANLEY (2003), "Chinese Media and Youth : Attitudes Toward Nationalism and Internationalism", in: LEE, CHIN-CHUAN (ed.), Chinese Media, Global Contexts, London: RoutledgeCurzon, 97-118.

SARFT (2002)，事业发展，2002年统计 (Enterpirse Development, 2002 Statistics)，Beijing: SARFT， http://www.sarft.gov.cn/manage/publishfile/51/1095.html [15.05.06].

SARFT (2004)，电规剧审管理规定 (Regulation on Television Drama Censorship Management)， Beijing: SARFT, http:// www.sarft.gov.cn/articles/2004/10/20/20070924103214320244.html [19.01.08].

SARFT (2006)，电影剧本 (梗概) 备案、电影片管理规定 (Regulation on Film Script (and Outline) Filing and Movie Management), Beijing: SARFT, http://www.sarft.gov.cn/manage/publishfile/20/3747.html [17.01.08].

SARFT (2007)，国家广电总局主要职能 (Main Functions of the SARFT), Beijing: SARFT, http://www.sarft.gov.cn/articles/2007/05/01/20070919194959740037.html [17.01.08].

SCHMIDT, MANFRED G. (1995), Wörterbuch zur Politik (Dictionary to Politics), Stuttgart: Kröner.

SEITER, ELLEN (1992), "Semiotics, Structuralism, and Television", in: ALLEN, ROBERT C. (ed.), Channels of Discourse, Reassembled : television and contemporary criticism, second edition, 2nd ed., London: Routledge, 31-66.

SNYDER, JOEL (1980), "Picturing Vision", in: MITCHEL, W. J. THOMAS (ed.), The Language of Images, 4th ed., Chicago: The University of Chicago, 219-246.

SOKAL, ALAN D. \& BRICMONT, JEAN (2003), Intellectual Impostures - Postmoderm Philosophers' Abuse of Science, London: Profile.

STOKER, GERRY (1998), "Governance as Theory : Five Propositions", International Social Science Journal 50/155: 17-28.

STRANGE, SUSAN (1996), The Retreat of the State : The Diffusion of Power in the World Economy, Cambridge: Cambridge University Press.

TANG, JILI (唐季礼) (2003), "南财女貌 (Talented Man, Beautiful Woman; NCNM)", 20 episodes, People's Republic of China: Xingguang International (北京里光国际传媒).

TAYLOR, PETER J. (1996), "The Modern Multiplicity of States", in: KOFMAN, ELEONORE \& YOUNGS, GILLIAN (eds.), Globalization: Theory and Practice, London: Pinter, 99-108.

"The Great Learning (Daxue 大学)", vii-354, in LEGGE, JAMES (ed.) (1983), The Chinese Classics, Taipei: SMC Reprint. 
THØGERSEN, STIG (forthcoming), "Educational Tradition", Brill's Encyclopaedia of China, Leiden: Koninklijke Brill.

VAN DIJK, TEUN A. (1993), "Principles of Critical Discourse Analysis", Discourse \& Society 4/2: 249-283.

VAN DIJK, TEUN A. (1995), "Esoteric Discourse Analysis", Discourse \& Society 6/1: 5-6.

VAN DIJK, TEUN A. (2002), "Structures of Discourse and Structures of Power", Communication Yearbook 12: 18-59.

WANG, LANZHU (王兰柱) (ed.) (2004), China TV Rating Yearbook 2004, Beijing: 华夏出版社 (Huaxia chubanshe).

WANG, LANZHU (王兰柱) (ed.) (2005), China TV Rating Yearbook 2005, Beijing: 中国传媒大 学出版社 (Zhongguo chuanmei daxue chubanshe).

WANG, LANZHU (王兰柱) (ed.) (2006), China TV Rating Yearbook 2006, Beijing: 中国传媒大 学出版社 (Zhongguo chuanmei daxue chubanshe).

WANG, LANZHU (王兰柱) (ed.) (2007), China TV Rating Yearbook 2007, Beijing: 中国传媒大 学出版社 (Zhongguo chuanmei daxue chubanshe).

WANG, YI (1996), "From Revolutionary Culture to Popular Culture : Chinese Literature and Television 1987-1991 (从革命文化到大众文化: 中国文学与电视 1987-1991)", 302, Perth， West Australia: Murdoch University.

WARNKE, MARTIN (1994), "Politische Ikonographie. Hinweise auf eine sichtbare Politik. (Political Iconography. Evidence of Visual Politics.)", in: LEGGEWIE, CLAUS (ed.), Wozu Politikwissenschaft? U'ber das Neue in der Politik. (Why Political Science? On Novelties in Politics.) Darmstadt: Wissenschaftliche Buchgesellschaft, 170-178.

WEBER, MAX (1980), Wirtschaft und Gesellschaft (Economy and Society), 5th rev. ed., Tubingen: Mohr Siebeck.

WEI, YONGZHENG (魏永征) \& LI, DANLIN (李丹林) (eds.)，(2005)，影视法导论－电影电视节 目制作人须知 (Introduction to Film And Television Laws - Notice for Film and Television Programme Producers), Shanghai: 复旦大学出版社 (Fudan daxue chubanshe).

XU，HANGZHOU (徐航州) \& XU, FAN (徐帆) (2006)，电枧书目类型学(Typology of TV Programmes), Hangzhou: 浙江大学出版社 (Zhejiang daxue chubanshe).

YAN, HUA (2005), TV Soaps Feature Good 'Emperor' Hu Jintao, Hong Kong: Asia Times Online, http://www.atimes.com/atimes/China/GC26Ad02.html [03.05.08].

YU, JIANHONG (俞剑虹) (2005)，"民营资本对中国电枧剧市场运作的影响 (The Influence of Private Capital on the Operations of the Chinese Television Drama Market)", Journal of Beijing Film Academy 27-34. 
ZENG, QINGRUI (曾庆瑞) (2002)，守望电规剧的棈神家园(Keeping Watch over the Spiritual Health of Native Television Drama Series), 1st ed., Beijing: 北京广播学院出版杜 (Beijing guangbo xueyuan chubanshe).

ZENG, QINGRUI (曾庆瑞) (2005)，守望电规剧的精神家园(Keeping Watch over the Spiritual Health of Native Television Drama Series), 2nd ed., Beijing: 北京广播学院出版社 (Beijing guangbo xueyuan chubanshe).

ZENG, QINGRUI (曾庆瑞) (2006)，守望电规剧的精神家园(Keeping Watch over the Spiritual Health of Native Television Drama Series), 3rd ed., Beijing: 北京广播学院出版社 (Beijing guangbo xueyuan chubanshe).

ZHA, JIANYING (1995), China Pop : How Soap Operas, Tabloids, and Bestsellers are Transforming a Culture, New York: The New Press.

Zhanguo Ce (战国策) (2001), Guangzhou: 广州出版社 (Guangzhou chubanshe).

ZHANG, GUOTAO (张国涛) (2005), '汉武大常 出了错司马迁宫刑后还留长须('Han Wu Da Di' Mistakenly Dipicted Sima Qian with a Long Beard after his Castration), Beijing: Sina.com, http://ent.sina.com.cn/v/m/2005-01-10/1209624611.htm] [24.07.08].

ZHANG, JUNHUA, WILLMANN, KATRIN \& DREIKOSEN, URSULA (2005),

"'Kulturwirtschaf' - Das letzte Stük Kuchen der Reform - Eine kritische Betrachtung der chinesischen Kulturpolitik und deren Praxis im Prozess der Globalisierung ('Creative Economy' - The Last Slice of the Reform Cake - A Critical Examination of Chinese Cultural Policies and their Practical Application in the Process of Globalization)", China aktuell 06/2005: 25-47.

ZHANG, MEI (1999), "From Lei Feng to Zhang Haidi : Changing Media Images of Model Youth in the Post-Mao Reform Era", in: KLUVER, RANDY \& POWERS, JOHN H. (eds.), Civic Discourse, Civil Society, and Chinese Communities, Stamford: Ablex Publishing Corporation, 111-123.

ZHANG, YINGJIN (张英进) (2002), Screening China : Critical Interventions, Cinematic Reconfigurations, and the Transnational Imaginary in Contemporary Chinese Cinema, Ann Arbor: Center for Chinese Studies, The University of Michigan.

ZHANG, YINGIN (张英进) (2004), "西方学界的中国电影研究方法选评 (Selected Review on the Research Methodologies of Chinese Film Studies Practiced in the Western Academic World)", 北京电影院学报 (Joumal Beijing Film Academy) 2004/5: 1-9.

ZHAO, BIN (1999), "Mouthpiece or Money-Spinner? The Double Life of Chinese Television in the Late 1990s." International Journal of Cultural Studies 2/3: 291-305.

ZHAO, YUEZHI (1998), Media, Market, and Democracy in China - Between the Party Line and the Bottom Line, Chicago: University of Illinois Press.

ZHAO, YUEZHI (2000), "From Commercialization to Conglomeration : The Transformation of the Chinese Press within the Orbit of the Party State", Journal of Communication 50/2: 3-26. 
ZHAO, YUEZHI (2003), "'Enter the World' : Neo-Liberal Globalization, the Dream for a Strong Nation, and the Chinese Press Discourses on the WTO", in: LEE, CHINCHUAN (ed.), Chinese Media, Global Contexts, London: RoutledgeCurzon, 32-56.

ZHAO, YUEZHI \& GUO, ZHENZHI (2005), "Television in China : History, Political Economy, and Ideology", in: WASKO, JANET (ed.), A Companion to Television, Oxford: Blackwell Publishing, 521-539.

ZHU, YING (2005), "Yongzheng Dynasty and Chinese Primetime Television Drama", Cinema Joumal Number 4: 3-17.

ZÜRN, MICHAEL (1998), Regieren jenseits des Nationalstaates : Globalisierung und Denationalisierung als Chance (Governance Beyond the Nation State: Globalization and Denationalization as an Opportunity), Frankfurt a. M.: Suhrkamp. 